\title{
Platinum-Catalyzed Domino Reaction with Benziodoxole Reagents for Accessing Benzene-Alkynylated Indoles
}

\author{
Yifan Li and Jerome Waser ${ }^{*[a]}$
}

Dedication ((optional))

\begin{abstract}
Indoles are omnipresent in natural products, bioactive molecules and organic materials. Consequently, their synthesis or functionalization are important fields of research in organic chemistry Most works focus on installation or modification of the pyrrole ring. To access benzene ring-functionalized indoles with an unsubstituted pyrrole ring remains more challenging. Herein we report a platinumcatalyzed cyclization-alkynylation domino process to obtain selectively C5- or C6- functionalized indoles starting from easily available pyrroles. Our work combines for the first time a platinum catalyst with EthynylBenziodoXole (EBX) hypervalent iodine reagents in a domino process for the synthesis of polyfunctionalized arene rings and gives access to important building blocks for the synthesis of bioactive compounds and organic materials.
\end{abstract}

Functionalized indoles occupy a privileged position in organic chemistry, as they can be found in biomolecules, drugs, agrochemicals and materials. ${ }^{[1]}$ To access these important building blocks, a first approach consists in the introduction of functional groups onto simple, commercially available indoles. In order to have a more efficient alternative to well-established cross-coupling reactions, the transition metal-catalyzed direct functionalization of $\mathrm{C}-\mathrm{H}$ bond on indoles has been intensively investigated in the last two decades. Nevertheless, this approach is mostly limited to the modification of the more reactive pyrrole ring (innate reactivity, Figure 1, A) ${ }^{[2]}$ The functionalization of the benzene ring has been only rarely achieved and is limited to C2,C3disubstituted indoles. ${ }^{[3]}$ One notable exception is the selective iridiumcatalyzed C7-borylation of indoles reported by Hartwig and coworkers. ${ }^{[4]}$ We were particularly interested by the introduction of an alkyne onto the benzyl ring of indoles, as alkynes are key building blocks in medicinal, synthetic and material chemistry. ${ }^{[5]}$ Direct alkynylation approaches can be used only to access 2- and 3substituted indoles. ${ }^{[6]}$ This is an important limitation, as 5- or 6alkynylated indoles can be found in bioactive compounds such as 5LOX (5-lipoxygenase) inhibitor $\mathbf{1}^{[7 \mathrm{7a}}$ or NMDA ( $N$-Methyl-DAspartate Receptor) antagonist 2. ${ }^{[7 b]}$ Alkynylated carbazoles are especially important for organic materials, such as macrocycle $\mathbf{3}$, an organic sensor for the detection of TNT. ${ }^{[7 c]}$ If an efficient method for the synthesis of benzene-alkynylated indoles can be developed, more complex compounds could then be easily obtained by using wellestablished $\mathrm{C}-\mathrm{H}$ functionalization at the $\mathrm{C} 2$ or $\mathrm{C} 3$ positions or transformations of the triple bond.

As $\mathrm{C}-\mathrm{H}$ metalation of the arene ring of indoles is extremely difficult in presence of the more reactive $\mathrm{C} 2$ and $\mathrm{C} 3 \mathrm{C}-\mathrm{H}$ bonds, we wondered if the desired reactive intermediate could be generated in situ during a metal-catalyzed cyclization step (Scheme 1). The use of such "domino" or "cascade" processes is one of the most efficient ways to increase molecular complexity in organic synthesis, ${ }^{[8]}$ but it had not yet been

[a] Mr. Yifan Li and Prof. Dr. Jerome Waser

Laboratory of Catalysis and Organic Synthesis

Ecole Polytechnique Fédérale de Lausanne

EPFL SB ISIC LCSO, BCH 4306, 1015 Lausanne, CH

E-mail: jerome.waser@eptl.ch

Homepage: http://lcso.epfl.ch/

Supporting information for this article is given via a link at the end of the document.((Please delete this text if not appropriate)) applied to the synthesis of benzene alkynylated indoles. In fact, reported cascade approaches by the group of Cacchi and Zhu for the synthesis of alkynylated indoles have focused on the construction of the pyrrole ring using palladium catalysis (Scheme 1, A).${ }^{[9]}$ To develop a domino process to access indoles functionalized on the benzene ring, another type of cyclization would be needed. In this respect, we were particularly attracted by the works of Alcaide, Hashmi, and Ma among others to form the benzene ring of heterocycles via the cyclization of alkynes ${ }^{[10]}$ using well-established $\pi$-activation catalysts such as gold and platinum. ${ }^{[1]}$ In fact, one single example of gold-catalyzed indole ring formation starting from easily available pyrrole ethers has been reported. ${ }^{[10 b]}$ This precedence gave solid basis for the cyclization step of the domino process, but the envisaged alkynylation appeared highly challenging, as the generated intermediates are known to broadly favor protodemetalation over further $\mathrm{C}-\mathrm{C}$ bond formation. The formation of a new $\mathrm{C}-\mathrm{C}$ bond on a $\mathrm{Csp}^{2}$ center of an indole arene ring after cyclization has to the best of our knowledge never been realized.
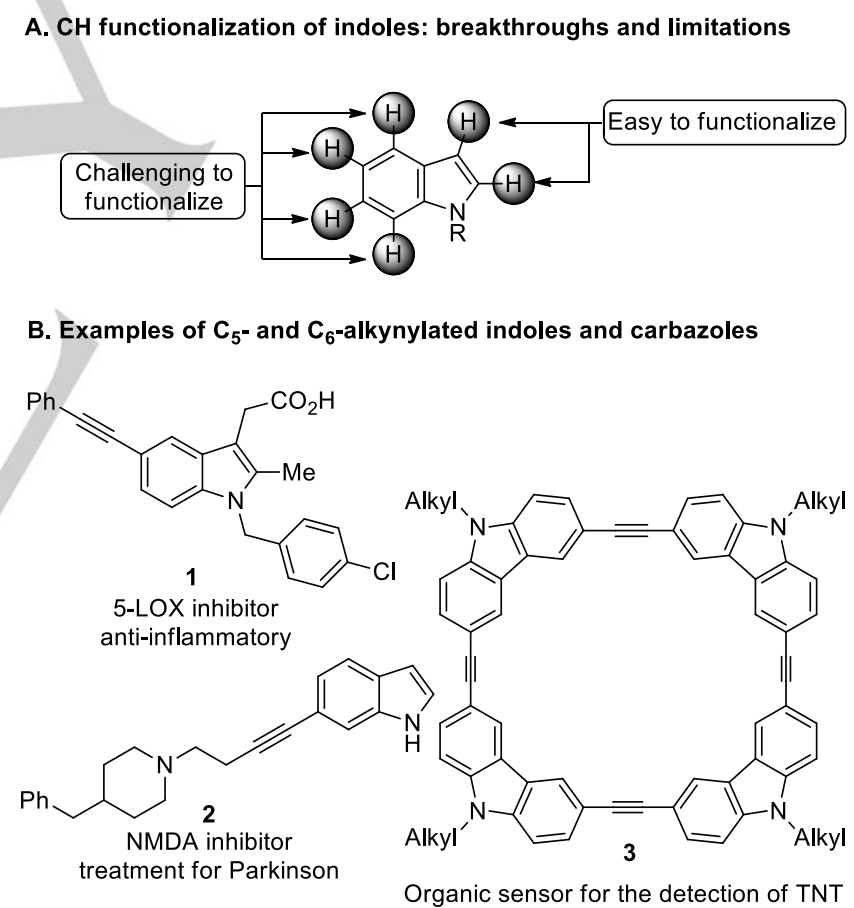

Figure 1. 5- and 6- alkynylated indoles: outside the scope of $\mathrm{C}-\mathrm{H}$ functionalization!

To solve the challenge associated with the low reactivity of gold intermediates in $\mathrm{C}-\mathrm{C}$ bond forming reactions, our group has turned to the exceptional reactivity of hypervalent iodine alkynylation reagents. ${ }^{[6 \mathrm{~b}-\mathrm{d}, 12]} \mathrm{We}$ recently reported the first successful domino process in the synthesis of 3-alkynylated furans by the cyclizationalkynylation of allene ketones. ${ }^{[13]}$ Although this work resulted in the synthesis of regioisomers difficult to access via $\mathrm{C}-\mathrm{H}$ functionalization, it was still limited to the more reactive heteroarene class of substrates. Herein, we report that the change to a platinum catalyst in combination with ethynylbenziodoxole reagent $\mathbf{4 a}^{[14]}$ led to a successful cyclizationalkynylation domino process starting from pyrrole homopropargylic ethers to give either C5 or C6-alkynylated indoles in good yields and high regioselectivity (Scheme $1, \mathbf{B}$ ). In addition to giving access to 
important building blocks and scaffolds, this new transformation represents also a breakthrough in the field of catalysis and hypervalent iodine chemistry, as it is the first example of a platinum-catalyzed process with EBX reagents.

\section{A. Previous domino processes: alkynylated pyrrole rings.}<smiles>[R]C#Cc1ccccc1N[R2]</smiles><smiles>[R]C#Cc1c([R])n([R1])c2ccccc12</smiles>

B. This work: domino process to access alkynylated benzene rings.

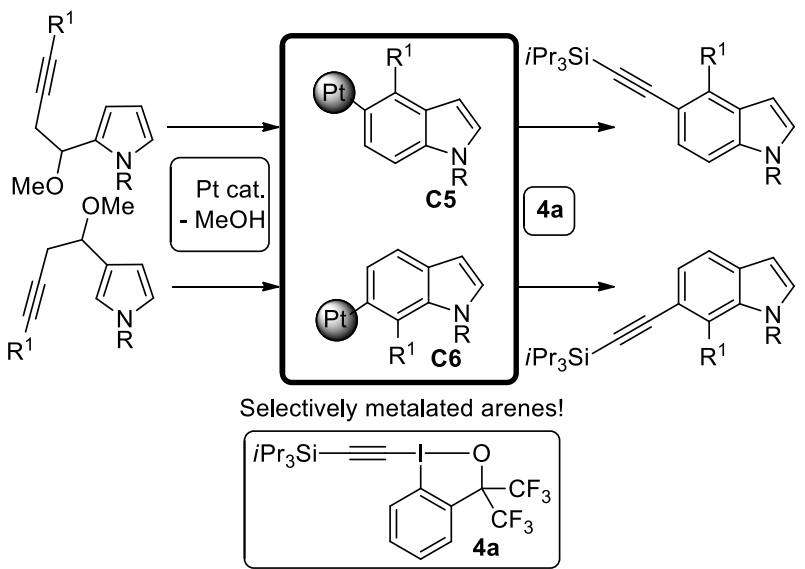

Scheme 1. Domino processes to access alkynylated indoles.

We started our investigation with pyrrole derivatives $\mathbf{5}$ and hypervalent iodine reagent 4a. Substrate $\mathbf{5}$ is easily accessible in two steps from commercially available compounds and the generation of only one molecule of water is required for aromatization. The previous catalytic conditions developed in our group for the domino cyclizationalkynylation of allene ketones ${ }^{[13]}$ were not suitable for the pyrrole system. After a broad screening of gold catalysts, the desired product could not be obtained. We then turned our attention to platinum catalysts (Table 1). ${ }^{[10 \mathrm{c}, 11 \mathrm{a}]}$ Preliminary results with $\mathrm{PtCl}_{2}$ were promising, as $13 \%$ of 5-ethynyl indole 8 a could be isolated with sodium carbonate as base (entry 1 ). Attempts to modulate the reactivity of the catalyst by formation of a more electrophilic platinum salt (entry 2) or using nitrogen-based ligands (entries 3 and 4) both suppressed the formation of 8a. In contrast, changing the base to sodium bicarbonate allowed increasing the yield to $40 \%$ (entry 5). Investigation of solvents showed that $\mathrm{CH}_{3} \mathrm{CN}$ was best (entries 6-8). Neither the use of other hypervalent iodine reagents, such as TIPS-EBX (4b) or alkynyliodonium salt 4c (entries 9-10), nor platinum(IV) or (0) catalysts (entries 11-12) led to improved results. At this point, we decided to investigate the influence of the group eliminated in the aromatization step. Changing the leaving group from hydroxy to methoxy led to a major increase in yield to $91 \%$ (entry 13). A silyl ether could also be used, ${ }^{[10 b]}$ but led to a lower yield (80\%, entry 14$)$ A bromide counterion led to a slightly higher yield (entry 15) and an iodide completely suppressed the reaction (entry 16). The results obtained with $\mathrm{PtCl}_{2}$ and $\mathrm{PtBr}_{2}$ were difficult to reproduce, probably due to the poor solubility of reagent $\mathbf{4 a}$ in $\mathrm{CH}_{3} \mathrm{CN}$. Using a co-solvent led to more reproducible results (entries 17-19). THF was the best, giving the desired product $8 \mathbf{a}$ in $96 \%$ yield (entry 18 ).

With optimized conditions in hand, we then examined the scope of the cyclization-ethynylation domino reaction (Table 2). Indole 8a was obtained in $84 \%$ isolated yield on a $0.3 \mathrm{mmol}$ scale (entry 1 ). Only C5ethynylation was observed. Modification of the substituent on the nitrogen was investigated first. A tolyl and a benzyl group were well tolerated (entries 2-3), as well as different substituents on an alkyl chain such as methoxy and trifluoromethyl (entries 4-5). Substitution of the alkyne was examined next. The desired C5-alkynylated product 8f was obtained in $70 \%$ yield with a methyl group (entry 6). With aromatic substituents, formation of the C6-alkynylated product $\mathbf{1 0}$ was observed for the first time. The C5-alkynylated product 8 was still the major product with a phenyl, a tolyl or a para-bromophenyl substituent (entries 7-9). The latter case also demonstrated that halogen substituents were tolerated under the reaction conditions, allowing further functionalization of the products via classical cross-coupling chemistry. With a naphthyl group, a 1:1 mixture of regioisomers was obtained (entry 10). With a methyl substituent in propargylic position, the C5-alkynylated product $\mathbf{8 k}$ was obtained in $75 \%$ yield (entry 11 ). In contrast, when tertiary ether $\mathbf{6} \mathbf{1}$ was used, the C6-alkynylated product 10e was formed (entry 12). Alkynylated carbazole $8 \mathbf{m}$ was obtained in $76 \%$ yield (entry 13). The domino process could also be applied to the synthesis of thiophene-fused heterocycle $\mathbf{8 n}$ in $78 \%$ yield (entry 14 ).

Table 1. Optimization of the Domino Cyclization-Alkynylation Reaction

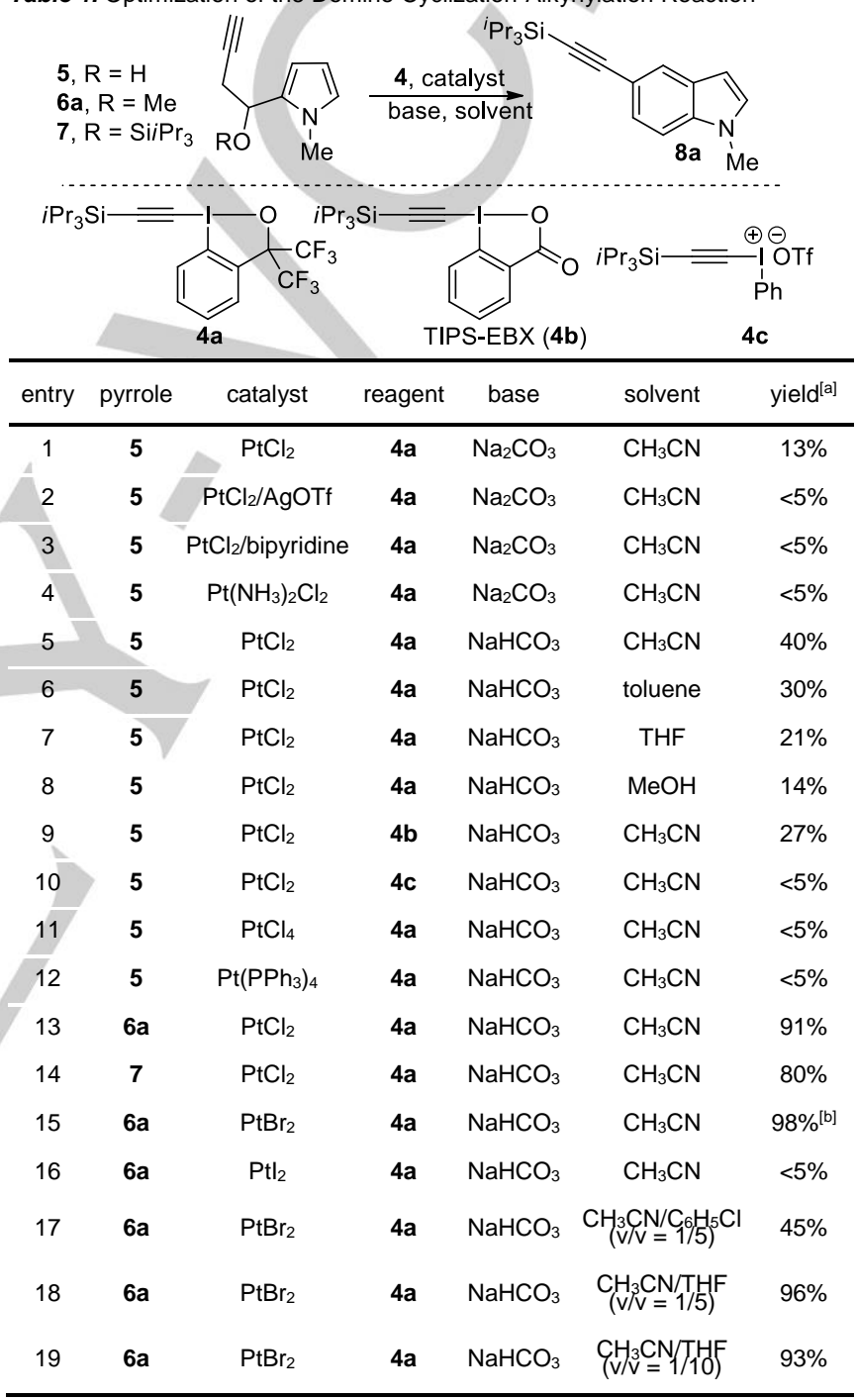

[a] Reaction condition: 5-7 $(0.10 \mathrm{mmol})$, catalyst $(10 \mathrm{~mol} \%), 4(0.20 \mathrm{mmol})$, base $(0.20 \mathrm{mmol}), 0.1 \mathrm{M}, \mathrm{RT}, 72 \mathrm{~h}$. Isolated yields after column chromatography. ${ }^{[\mathrm{b}]}$ Not reproducible.

Having achieved the synthesis of C5-alkynylated indoles starting from C2-substituted pyrroles, we then turned to $\mathrm{C} 3$-substituted derivatives to achieve C6 functionalization. (Table 3). Pyrrole 9a bearing a terminal alkyne gave C6-alkynylated indole 10a exclusively in $77 \%$ yield (entry 1). Substituted alkynes could also be used to access 6,7-disubstituted indoles in 47-53\% yield (entries 2-4). Starting from tertiary ethers, 4,6disubstituted products could be obtained (entries 5 and 6). In this case, the same products were obtained as when starting from $\mathrm{C} 2$-substituted pyrroles. We then wondered if more complex trisubstituted indoles could be accessed. In fact, 4,6,7- and 4,5,6-substituted indoles $\mathbf{1 0 g}$ an 10h could be obtained in $44 \%$ and $34 \%$ yield respectively (entries 7 and 8). Although the yields are moderate, the synthesis of these polysubstituted compounds using another method would have been 
extremely difficult. Finally, the synthesis of C6-alkynylated carbazoles 10i-j was also possible (entries 9 and 10).

Table 2. Scope of 5-Alkynylated Indoles.

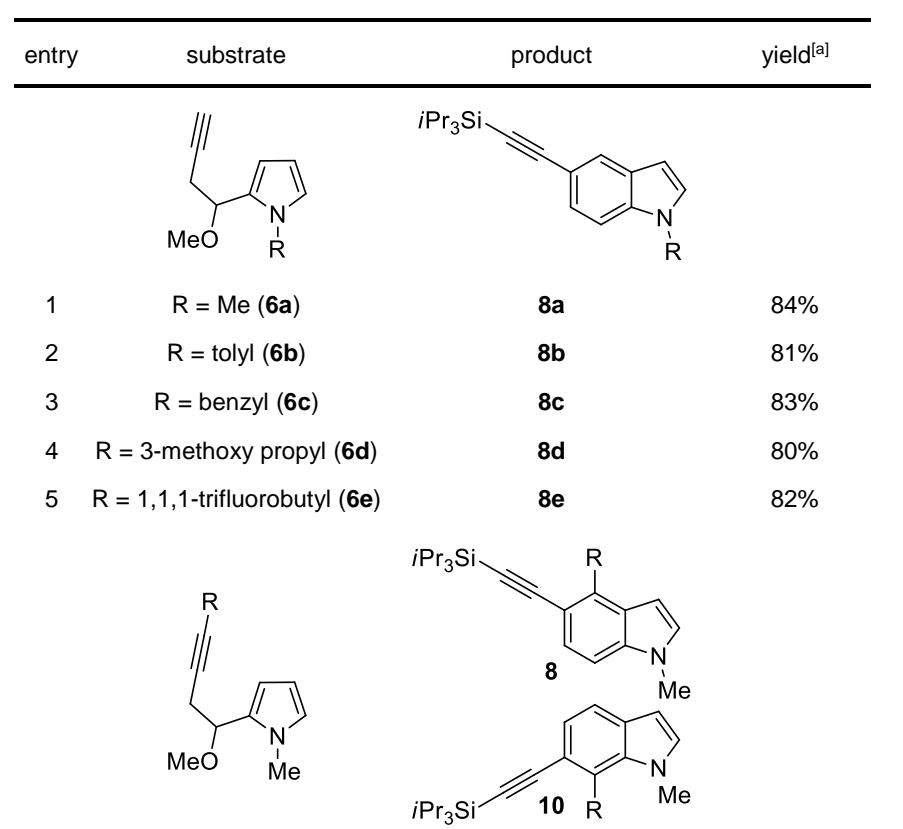

$\begin{array}{cc}6 & \mathrm{R}=\text { Me }(\mathbf{6 f}) \\ 7 & \mathrm{R}=\text { phenyl }(\mathbf{6 g}) \\ 8 & \mathrm{R}=\text { tolyl }(\mathbf{6 h}) \\ 9 & \mathrm{R}=\text { p-Br-phenyl }(6 \mathbf{i}) \\ 10 & \mathrm{R}=\text { naphthyl }(6 \mathbf{j})\end{array}$

$8 f$

$8 \mathrm{~g} / 10 \mathrm{c}(4 / 1)$

8h/10k $(3 / 1)$

$8 \mathrm{i} / 10 \mathrm{l}(2.5 / 1)$

8j/10d $(1 / 1)$

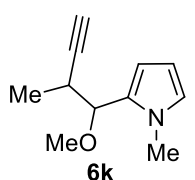

12<smiles>C#CCC(C)(OC)c1cccn1C</smiles><smiles>C#CCC(OC)c1cc2ccccc2n1C</smiles>

$6 \mathrm{~m}$<smiles>Cc1cc2c(ccn2C)cc1C#C[SiH2]C(C)C</smiles><smiles>Cc1cc(C#C[SiH2]C(C)C)cc2c1ccn2C</smiles>

$70 \%$

$70 \%(56 \%)^{[b]}$

$69 \%(52 \%)^{[\mathrm{b}]}$

$62 \%(44 \%)^{[b]}$

$31 \% / 31 \%$

$75 \%$
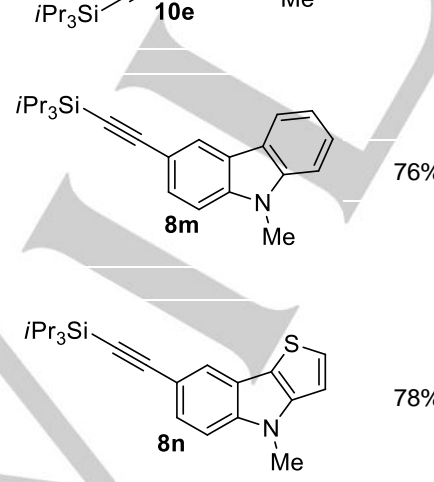

1

cyclization could occur either on the $\mathrm{C} 2$ or $\mathrm{C} 3$ position of the pyrrole ring to give intermediates II and IV respectively. Attack on the more nucleophilic C2 position, leading to II is more probable. From II, two different pathways are possible: 1,2 shift of the Pt-bearing vinyl group via platinum carbene-cyclopropane intermediate III $^{[11 a, 15]}$ to give carbocation IV, or 1,2-shift of the alkoxy substituent to give intermediate VII. ${ }^{[10]}$ C5- or C6- metalated intermediates V and VIII will then be generated from IV and VII respectively after deprotonation and aromatization via methanol elimination. Alkynylated products $\mathbf{8}$ and $\mathbf{1 0}$ can be formed by reaction of EBX reagent $\mathbf{4 a}$ with the organoplatinum intermediates. As this point, it is not clear if the alkynylation step proceeds via an oxidative addition/reductive elimination mechanism on platinum ${ }^{[16]}$ or by direct nucleophilic attack of the organometallic intermediates onto reagent 4a.

Table 3. Scope of 6-Alkynylated Indoles.

entry substrate $\quad$ product $\quad$ yield ${ }^{[\mathrm{a}]}$

$77 \%$

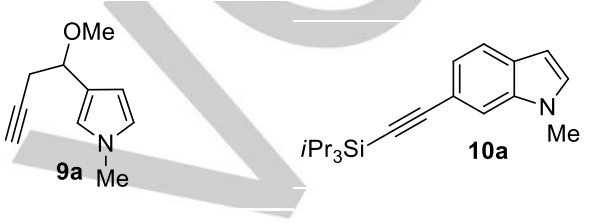

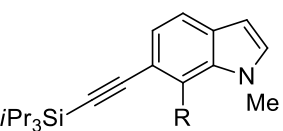

$10 b$

$49 \%$

$53 \%$

$47 \%$

10c

$10 d$<smiles>[R]c1cc(C#C[SiH2]C(C)C)cc2c1ccn2C</smiles>

$10 \mathrm{e}$

$54 \%$

$\mathrm{R}=\mathrm{Me}(\mathbf{9 e})$

$10 f$

$65 \%$<smiles>Cc1cc(C#CC(C)C)c(C)c2c1ccn2C</smiles>

$44 \%$

$76 \%$<smiles>CC#CCC(C)(OC)c1ccn(C)c1</smiles>

8<smiles>C#CC(C)C(C)(OC)c1ccn(C)c1</smiles>

$78 \%$<smiles>[R]c1ccc2c(c1)c(C(CC#C)OC)cn2C</smiles>

$\mathrm{R}=\mathrm{H}(9 \mathrm{i})$

$10 i$<smiles>Cc1c(C#C[SiH2]C(C)C)cc2c(ccn2C)c1C</smiles>

$34 \%$<smiles>[R]c1ccc2c(c1)c1ccc(C#C[SiH2]C(C)C)cc1n2C</smiles>

4a $(0.60 \mathrm{mmol}), \mathrm{NaHCO}_{3}(0.60 \mathrm{mmol}), \mathrm{THF} / \mathrm{CH}_{3} \mathrm{CN}(3 \mathrm{~mL}, \mathrm{v} / \mathrm{v}=5 / 1), 72-120 \mathrm{~h}$, isolated yields after column chromatography are given. ${ }^{[b]} Y$ Yeld determined by ${ }^{1} \mathrm{H}$ NMR. The isolated yield of isomer $\mathbf{8}$ is given in parenthesis.

The developed domino process is highly complex. Consequently, any proposal about the reaction mechanism is highly speculative at this stage of development. Based on the well-established $\pi$-acidity of platinum, ${ }^{[11 a]}$ the first step of the catalytic cycle is most probably activation of the alkyne to give complex I (Scheme 2). At this point,

10j

$10 \mathrm{R}=\mathrm{OMe}(\mathbf{9 j})$

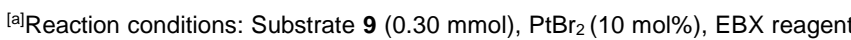
4a $(0.60 \mathrm{mmol}), \mathrm{NaHCO}_{3}(0.60 \mathrm{mmol}), \mathrm{THF} / \mathrm{CH}_{3} \mathrm{CN}(3 \mathrm{~mL}, \mathrm{v} / \mathrm{v}=5 / 1), 72-120 \mathrm{~h}$, isolated yields after column chromatography are given. 
If the $\mathrm{C} 3$-substituted pyrrole is used as starting material, the mechanism is simpler as $\mathrm{C} 2$-attack of the indole leads directly to a 6 membered ring and therefore to exclusive formation of the C6alkynylated product 10. The observed regioselectivity for secondary ethers is in accordance with a shift of the vinyl group. In contrast, shift of the alkoxy group had been observed in the cyclization step when using gold catalysts. ${ }^{[10 \mathrm{~b}]}$ This may be due to an easier formation of platinum carbene intermediate III when compared to gold catalysts. ${ }^{[17}$ This result further emphasizes an advantage of the platinum catalyst, as with gold both C2- and C3-substituted pyrroles would have given C6alkynylated products. With pyrroles substituted with tertiary ethers, C6-alkynylated products were observed independent of the structure of the starting material. In this case, the presence of the methyl group may further stabilize transition state VI and favor the shift of the alkoxy group

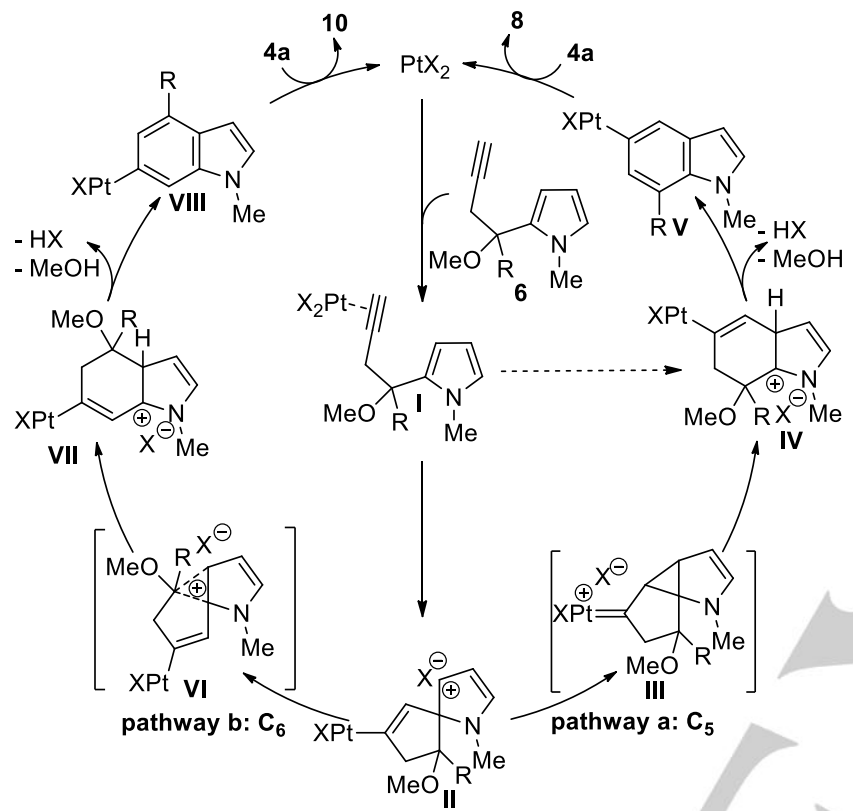

Scheme 2. Speculative mechanism for the domino cyclization-alkynylation.

The obtained alkynylated indoles are interesting building blocks, as the unsubstituted pyrrole ring can be easily further derivatized using established methods and the terminal alkyne obtained after silyl group removal can serve as platform for further functionalization. For example, deprotection of alkyne $\mathbf{8 a}$ followed by gold-catalyzed selective C3 alkynylation with TIPS-EBX $(\mathbf{4 b})^{[6 b]}$ gave monoprotected diyne 11, ready for further selective derivatization (Scheme 3, A). Starting from naphthyl derivative 10d, deprotection followed by platinum-catalyzed cycloisomerization ${ }^{[18]}$ gave access to helicene $\mathbf{1 2}$ (Scheme 3, B). Helicenes are important molecules in asymmetric catalysis and material science, but have never been synthesized with a terminal pyrrole ring to the best of our knowledge. ${ }^{[19]}$
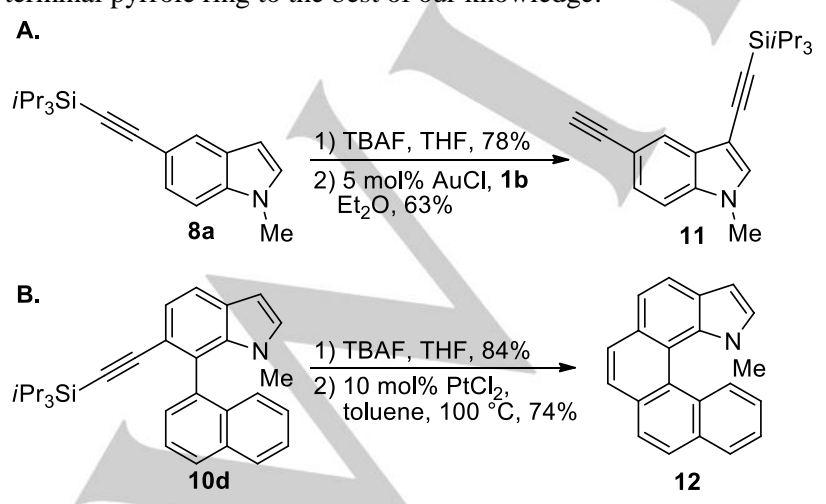

Scheme 3. Further transformations of the alkynylated indole products.

In conclusion, we have reported a new domino cyclization strategy for the efficient synthesis of C5- or C6- alkynylated indoles starting from easily available $\mathrm{C} 2$ - or $\mathrm{C} 3$-substituted pyrroles. Our strategy is complementary to $\mathrm{C}-\mathrm{H}$ functionalization, as the benzene positions on indole are difficult to modify directly, due to the higher reactivity of the pyrrole ring. Key for success was the unprecedented combination of a platinum catalyst with EthynylBenziodoXole (EBX) reagents. The domino process realized in this work represents a new general strategy for the synthesis of alkynylated arenes. Further investigations will focus on the synthesis of other (hetero)arenes and on the introduction of different functional groups in the final bond forming step. ${ }^{[20]}$

\section{Acknowledgements}

EPFL and F. Hoffmann-La Roche Ltd are acknowledged for financial support and Alfa Aesar and Johnson Matthey for a gift of $\mathrm{PtCl}_{2}$.

Keywords: Domino Reactions, Alkynes, Indoles, Hypervalent lodine, Platinum Catalysis.

[1] a) R. J. Sundberg, Indoles, Academic Press, New York, 1996; b) A F. Pozharskii, A. R. Katritzky, A. T. Soldatenkov, Heterocycles in Life and Society: An Introduction to Heterocyclic Chemistry, Biochemistry, and Applications, Chichester, West Sussex, 2011.

[2] Selected examples: a) B. S. Lane, D. Sames, Org. Lett. 2004, 6, 2897 ; b) B. S. Lane, M. A. Brown, D. Sames, J. Am. Chem. Soc. 2005, 127, 8050; c) N. P. Grimster, C. Gauntlett, C. R. A. Godfrey, M. J. Gaunt, Angew. Chem., Int. Ed. 2005, 44, 3125; d) R. J. Phipps, N. P. Grimster, M. J. Gaunt, J. Am. Chem. Soc. 2008, 130, 8172; e) N. R. Deprez, D. Kalyani, A. Krause, M. S. Sanford, J. Am. Chem. Soc. 2006, 128, 4972.

[3] a) G. Q. Yang, P. Lindovska, D. J. Zhu, J. Kim, P. Wang, R. Y Tang, M. Movassaghi, J. Q. Yu, J. Am. Chem. Soc. 2014, 136, 10807; b) H. Liu, C. Zheng, S.-L. You, J. Org. Chem 2014, 79, 1047

[4] D. W. Robbins, T. A. Boebel, J. F. Hartwig, J. Am. Chem. Soc. 2010 132, 4068.

[5] F. Diederich, P. J. Stang, R. R. Tykwinski, Acetylene Chemistry Chemistry, Biology and Material Science, F. Diederich, P. J. Stang, R. R. Tykwinski ed., Wiley-VCH, 2005.

[6] a) Y. H. Gu, X. M. Wang, Tetrahedron Lett. 2009, 50, 763; b) J. P. Brand, J. Charpentier, J. Waser, Angew. Chem., Int. Ed. 2009, 48, 9346; c) J. P. Brand, C. Chevalley, J. Waser, Beilstein J. Org. Chem. 2011, 7, 565; d) J. P. Brand, C. Chevalley, R. Scopelliti, J. Waser, Chem. Eur. J. 2012, 18, 5655; e) G. L. Tolnai, S. Ganss, J. P. Brand, J. Waser, Org. Lett. 2013, 15, 112; f) T. de Haro, C. Nevado, J. Am. Chem. Soc. 2010, 132, 1512; g) L. Yang, L. A. Zhao, C. J. Li, Chem. Commun. 2010, 46, 4184

[7] a) M. Elkady, R. Nieß, A. M. Schaible, J. Bauer, S. Luderer, G. Ambrosi, O. Werz, S. A. Laufer, J. Med. Chem. 2012, 55, 8958; b) J L. Wright, T. F. Gregory, S. R. Kesten, P. A. Boxer, K. A. Serpa, L. T. Meltzer, L. D. Wise, S. A. Espitia, C. S. Konkoy, E. R Whittemore, R. M. Woodward, J. Med. Chem. 2000, 43, 3408; c) T. Naddo, Y. Che, W. Zhang, K. Balakrishnan, X. Yang, M. Yen, J. Zhao, J. S. Moore, L. Zang, J. Am. Chem. Soc. 2007, 129, 6978.

[8] L. F. Tietze, Chem. Rev 1996, 96, 115.

[9] a) A. Arcadi, S. Cacchi, G. Fabrizi, F. Marinelli, L. M. Parisi, J. Org. Chem. 2005, 70, 6213; b) B. Yao, Q. Wang, J. P. Zhu, Angew. Chem., Int. Ed. 2012, 51, 12311. Review on cascade reactions to access indoles: J. Barluenga, F. Rodriguez, F. J. Fananas, Chem. Asian J. 2009, 4, 1036

[10] a) B. Alcaide, P. Almendros, J. M. Alonso, M. T. Quiros, P. Gadzinski, Adv. Synth. Catal. 2011, 353, 1871; b) A. S. K. Hashmi, W. B. Yang, F. Rominger, Chem. Eur. J. 2012, 18, 6576; c) Y. A. Qiu, W. Q. Kong, C. L. Fu, S. M. Ma, Org. Lett. 2012, 14, 6198.

[11] a) A. Fürstner, P. W. Davies, Angew. Chem., Int. Ed. 2007, 46, 3410; b) A. S. K. Hashmi, Chem. Rev 2007, 107, 3180; c) D. J. Gorin, F. D. Toste, Nature 2007, 446, 395.

[12] a) F. M. Beringer, S. A. Galton, J. Org. Chem. 1965, 30, 1930; b) M. Ochiai, T. Ito, Y. Takaoka, Y. Masaki, M. Kunishima, S. Tani, Y Nagao, J. Chem. Soc., Chem. Commun. 1990, 118; c) V. V. Zhdankin, P. J. Stang, Tetrahedron 1998, 54, 10927.

[13] Y. Li, J. P. Brand, J. Waser, Angew. Chem., Int. Ed. 2013, 52, 6743.

[14] V. V. Zhdankin, C. J. Kuehl, A. P. Krasutsky, J. T. Bolz, A. J. Simonsen, J. Org. Chem. 1996, 61, 6547. 
[15] a) N. Chatani, N. Furukawa, H. Sakurai, S. Murai, Organometallics 1996, 15, 901; b) N. Chatani, K. Kataoka, S. Murai, N. Furukawa, Y. Seki, J. Am. Chem. Soc. 1998, 120, 9104; c) S. I. Lee, N. Chatani, Chem. Comm. 2009, 371; d) A. Fürstner, H. Szillat, B. Gabor, R. Mynott, J. Am. Chem. Soc. 1998, 120, 8305; e) A. Fürstner, H. Szillat, F. Stelzer, J. Am. Chem. Soc. 2000, 122, 6785; f) B. MartínMatute, D. J. Cárdenas, A. M. Echavarren, Angew. Chem., Int. Ed. 2001, 40, 4754

[16] a) A. J. Canty, R. P. Watson, S. S. Karpiniec, T. Rodemann, M. G. Gardiner, R. C. Jones, Organometallics 2008, 27, 3203; Selected examples of reaction of Pt-aryl intermediates with alkynes: b) M. E. Cucciolito, A. d. Renzi, G. Roviello, F. Ruffo, Organometallics 2008 , 27, 1351; c) J. R. Berenguer, J. Fernández, N. Giménez, E. Lalinde, M. T. Moreno, S. Sánchez, Organometallics 2013, 32, 3943.

[17] Y. Qiu, D. Ma, W. Kong, C. Fu, S. Ma, Org. Chem. Frontiers 2014 1,62 .

[18] a) V. Mamane, P. Hannen, A. Fürstner, Chem. Eur. J. 2004, 10 , 4556; b) J. Storch, J. Sykora, J. Cermak, J. Karban, I. Cisarova, A. Ruzicka, J. Org. Chem. 2009, 74, 3090.

[19] Y. Shen, C. F. Chen, Chem. Rev. 2012, 112, 1463.

[20] The use of aryliodonium salts, Togni's reagent and cyano- or azidobenziodoxolones under the optimized reaction conditions did not result in the formation of the domino cyclization-functionalization products. The design of new catalyst systems and reagents will be required in the future. 
Entry for the Table of Contents

COMMUNICATION

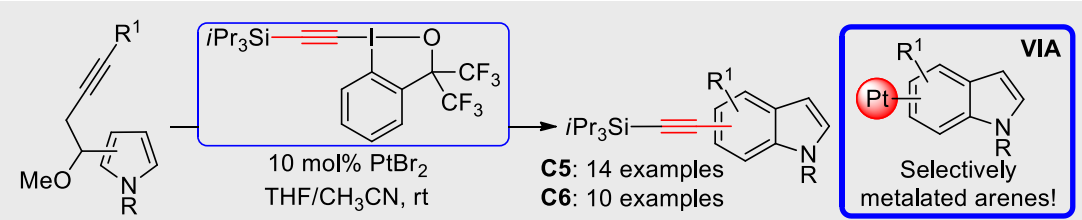

Yifan Li and Jerome Waser*

Page No. - Page No.

Platinum-Catalyzed Domino Reaction with Benziodoxole Reagents for Accessing Benzene-Alkynylated Indoles 


\section{Table of Contents}

1. General Methods $\quad$ S2

2. Preparation of Reagents $\quad$ S3

3. Synthesis of Starting Materials $\quad$ S8

$\begin{array}{ll}\text { 4. Cyclization-alkynylation domino process } & \text { S20 }\end{array}$

5. Further Functionalization $\quad$ S34

8. Spectra for New Compounds $\quad$ S36 


\section{General method}

All reactions were carried out in oven dried glassware under an atmosphere of nitrogen, unless stated otherwise. For quantitative flash chromatography technical grade solvents were used. For flash chromatography for analysis, HPLC grade solvents from Sigma-Aldrich were used. THF, $\mathrm{Et}_{2} \mathrm{O}, \mathrm{CH}_{3} \mathrm{CN}$, toluene, hexane and $\mathrm{CH}_{2} \mathrm{Cl}_{2}$ were dried by passage over activated alumina under nitrogen atmosphere $\left(\mathrm{H}_{2} \mathrm{O}\right.$ content $<10 \mathrm{ppm}$, Karl-Fischer titration $)$. $\mathrm{NEt}_{3}$ and pyridine were distilled under nitrogen from $\mathrm{KOH}$. $\mathrm{PtBr}_{2}$ was purchased from Aldrich, crushed into fine powder and kept in desiccator under anhydrous condition (decrease of reactivity has been observed for catalyst if prolonged exposition to air ( $c a 1$ month). The solvents were degassed by Freeze-Pump-Thaw method when mentioned. All chemicals were purchased from Acros, Aldrich, Fluka, VWR, Aplichem or Merck and used as such unless stated otherwise. Chromatographic purification was performed as flash chromatography using Macherey-Nagel silica 40-63, $60 \AA$, using the solvents indicated as eluent with 0.1-0.5 bar pressure.TLC was performed on Merck silica gel $60 \mathrm{~F}_{254}$ TLC glass plates or aluminium plates and visualized with UV light, permanganate stain, CAN stain or Anisaldehyde stain. Melting points were measured on a Büchi B-540 melting point apparatus using open glass capillaries, the data is uncorrected. ${ }^{1} \mathrm{H}-\mathrm{NMR}$ spectra were recorded on a Brucker DPX-400 $400 \mathrm{MHz}$ spectrometer in chloroform-d, DMSO- $\mathrm{d}_{6}$ or $\mathrm{CD}_{3} \mathrm{OD}$, all signals are reported in ppm with the internal chloroform signal at $7.26 \mathrm{ppm}$, the internal DMSO signal at $2.50 \mathrm{ppm}$ or the internal methanol signal at $3.30 \mathrm{ppm}$ as standard. The data is being reported as $(\mathrm{s}=$ singlet, $\mathrm{d}$ $=$ doublet, $\mathrm{t}=$ triplet, $\mathrm{q}=$ quadruplet, $\mathrm{qi}=$ quintet, $\mathrm{m}=$ multiplet or unresolved, $\mathrm{br}=$ broad signal, app $=$ apparent, coupling constant(s) in $\mathrm{Hz}$, integration, interpretation). ${ }^{13} \mathrm{C}$ NMR spectra were recorded with ${ }^{1} \mathrm{H}$-decoupling on a Brucker DPX-400 $100 \mathrm{MHz}$ spectrometer in chloroform-d, DMSO- $\mathrm{d}_{6}$ or $\mathrm{CD}_{3} \mathrm{OD}$, all signals are reported in ppm with the internal chloroform signal at $77.0 \mathrm{ppm}$, the internal DMSO signal at $39.5 \mathrm{ppm}$ or the internal methanol signal at $49.0 \mathrm{ppm}$ as standard. Infrared spectra were recorded on a JASCO FT-IR B4100 spectrophotometer with an ATR PRO410-S and a ZnSe prisma and are reported as $\mathrm{cm}^{-}$

${ }^{1}$ ( $\mathrm{w}=$ weak, $\mathrm{m}=$ medium, $\mathrm{s}=$ strong, $\mathrm{br}=$ broad $)$. Gas chromatographic and low resolution mass spectrometric measurements were performed on a Perkin-Elmer Clarus 600 gas chromatographer and mass spectrometer using a Perkin-Elemer Elite fused silica column (length: $30 \mathrm{~m}$, diameter: $0.32 \mathrm{~mm}$ ) and Helium as carrier gas. High resolution mass spectrometric measurements were performed by the mass spectrometry service of ISIC at the EPFL on a MICROMASS (ESI) Q-TOF Ultima API. HPLC measurement were done on a JASCO HPLC system with an AS2055 Autosampler, a PU 2089 Pump, a UV 2075 detector and a SEDEX 85 (SEDERE) detector using a CHIRALPAK IC column from DAICEL Chemical Industries Ltd. HPLC grade solvents from Sigma-Aldrich were used. 


\section{Preparation of Reagents}

\section{Triisopropylsilyl trimethylsilylacetylene (14)}

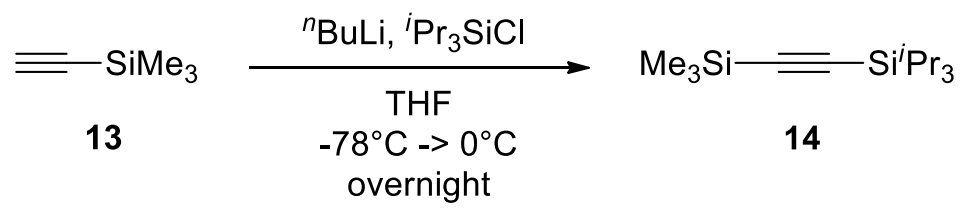

Following a reported procedure, ${ }^{[1]} n$-butyllithium $(2.5 \mathrm{~m}$ in hexanes, $12.0 \mathrm{~mL}, 29.9 \mathrm{mmol}$, 0.98 equiv) was added dropwise to a stirred solution of ethynyltrimethylsilane (13) $(3.0 \mathrm{~g}, 30$ mmol, 1.0 equiv) in THF $(48 \mathrm{~mL})$ at $-78^{\circ} \mathrm{C}$. The mixture was then warmed to $0{ }^{\circ} \mathrm{C}$ and stirred for $5 \mathrm{~min}$. The mixture was then cooled back to $-78^{\circ} \mathrm{C}$ and chlorotriisopropylsilane $(6.4 \mathrm{~mL}$, $30 \mathrm{mmol}, 1.0$ equiv) was added dropwise. The mixture was then allowed to warm to room temperature and stirred overnight. A saturated solution of ammonium chloride $(40 \mathrm{~mL})$ was added, and the reaction mixture was extracted with diethyl ether $(2 \times 60 \mathrm{~mL})$. The organic layer was washed with water and brine, then dried over $\mathrm{MgSO}_{4}$, filtered and concentrated under reduced pressure to obtain a colorless liquid which was further purified by Kugelrohr distillation $\left(56-57^{\circ} \mathrm{C} / 0.25 \mathrm{mmHg}\right)$ to yield $14(7.16 \mathrm{~g}, 28.0 \mathrm{mmol}, 92 \%$ yield) as a colorless liquid.

${ }^{1} \mathrm{H}$ NMR (400 MHz, $\left.\mathrm{CDCl}_{3}\right) \delta 1.08$ (m, $\left.21 \mathrm{H}, \mathrm{TIPS}\right), 0.18$ (s, $\left.9 \mathrm{H}, \mathrm{TMS}\right)$. IR v 2959 (m), 2944 (m), 2896 (w), 2867 (m), 1464 (w), 1385 (w), 1250 (m), 996 (w), 842 (s), 764 (s), 675 (m), $660(\mathrm{~m})$. Characterization data of $\mathbf{1 4}$ corresponded to the literature values. ${ }^{[1]}$

\section{1-Chloro-1,3-dihydro-3,3-bis(trifluoromethyl)-1,2-benziodoxole (17)}<smiles>OC(c1ccccc1)(c1ccccc1)C(F)(F)F</smiles>

15

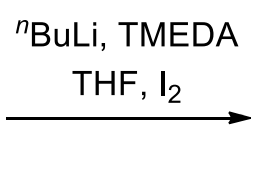<smiles>OC(c1ccccc1I)(C(F)(F)F)C(F)(F)F</smiles>

16

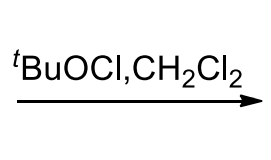<smiles>FC(F)(F)C1(C(F)(F)F)OI(Cl)c2ccccc21</smiles>

17

Following a reported procedure, ${ }^{[2]}$ TMEDA (distilled over $\left.\mathrm{KOH}\right)(1.26 \mathrm{~mL}, 8.20 \mathrm{mmol}, 0.2$ equiv) was added to a solution of ${ }^{n} \mathrm{BuLi}(2.5 \mathrm{M}$ in hexanes, $36.6 \mathrm{~mL}, 91.6 \mathrm{mmol}, 2.2$ equiv). After $15 \mathrm{~min}$, the cloudy solution was cooled to $0{ }^{\circ} \mathrm{C}$ and $15(7.0 \mathrm{~mL}, 42 \mathrm{mmol}, 1$ equiv) in THF (6 mL) was added dropwise. The reaction was stirred $30 \mathrm{~min}$ at $0{ }^{\circ} \mathrm{C}$ and then at $\mathrm{RT}$ overnight. $\mathrm{I}_{2}\left(11.2 \mathrm{~g}, 44.0 \mathrm{mmol}, 1.06\right.$ equiv) was then added portion wise at $0{ }^{\circ} \mathrm{C}$ and the mixture stirred at $0{ }^{\circ} \mathrm{C}$ for $30 \mathrm{~min}$ and $4 \mathrm{~h}$ at $\mathrm{RT}$. The reaction was quenched with saturated $\mathrm{NH}_{4} \mathrm{Cl}$. Et ${ }_{2} \mathrm{O}(100 \mathrm{~mL})$ was added and the layers were separated. The aqueous layer was then extracted twice with $\mathrm{Et}_{2} \mathrm{O}(3 \times 50 \mathrm{~mL})$. The organic layers were combined, washed twice with saturated $\mathrm{NaS}_{2} \mathrm{O}_{3}(2 \times 50 \mathrm{~mL})$, dried over $\mathrm{MgSO}_{4}$, filtered and reduced to afford $15.6 \mathrm{~g}$ of 16 as an brown oil which was used without further purification.

[1] C J. Helal, P A. Magriotis, E J. Corey, J. Am. Chem. Soc. 1996, 118, 10938.

[2].E. F. Perozzi, R. S. Michalak, G. D. Figuly, W. H. Stevenson, D. B. Dess, M. R. Ross, J. C. Martin, J. Org. Chem. 1981, 46, 1049. 
The crude oil was dissolved in wet $\mathrm{CH}_{2} \mathrm{Cl}_{2}(40 \mathrm{~mL})$ in the dark under air. ${ }^{t} \mathrm{BuOCl}(5.2 \mathrm{~mL}, 44$ mmol, 1.05 equiv) was then added dropwise at $0{ }^{\circ} \mathrm{C}$. After $30 \mathrm{~min}$, the resulting suspension was filtered to afford $17(7.30 \mathrm{~g}, 18.1 \mathrm{mmol}, 43 \%)$ as a yellow solid. The mother liquors were carefully reduced to one third and filtered to afford $17(3.51 \mathrm{~g}, 8.71 \mathrm{mmol}, 21 \%)$ as a yellow solid. Combined yield: $64 \%$. Mp $167-169^{\circ} \mathrm{C} .{ }^{1} \mathrm{H}$ NMR $\left(400 \mathrm{MHz}, \mathrm{CDCl}_{3}\right) \delta 8.09(\mathrm{~d}, 1 \mathrm{H}, J$ $=8.4 \mathrm{~Hz}, \mathrm{ArH}), 7.85(\mathrm{~m}, 1 \mathrm{H}, \mathrm{ArH}), 7.73(\mathrm{~m}, 2 \mathrm{H}, \mathrm{ArH}) .{ }^{13} \mathrm{C} \mathrm{NMR}\left(101 \mathrm{MHz}, \mathrm{CDCl}_{3}\right) \delta$ 133.8, 132.1, 131.6, 129.7, 128.5, 122.8 (q, $289 \mathrm{~Hz}), 113.4$, 84.8. Consistent with reported values. $^{[2]}$

\section{1-Hydroxy-3,3-bis(trifluoromethyl)-3-(1H)-1,2-benziodoxole (18)}

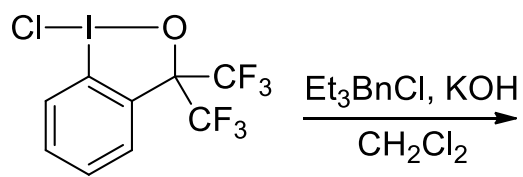

17<smiles>OI1OC(C(F)(F)F)(C(F)(F)F)c2ccccc21</smiles>

18

Following a preported procedure, ${ }^{[3]} \mathrm{Et}_{3} \mathrm{BnNCl}$ ( $83 \mathrm{mg}, 0.36 \mathrm{mmol}, 0.05$ equiv) was added to a stirring solution of 17 (10.7 g, $26.5 \mathrm{mmol}, 1$ equiv) in $\mathrm{CH}_{2} \mathrm{Cl}_{2}(150 \mathrm{~mL})$ and $\mathrm{KOH}(1.48 \mathrm{~g}$, $26.5 \mathrm{mmol}, 1$ equiv) in water $(28 \mathrm{~mL})$. The reaction was kept under air until TLC indicated that all starting material was consumed. The organic layer was separated and dried over $\mathrm{MgSO}_{4}$. The resulting solid was purified over a silica plug eluting with EtOAc, then recristallized in EtOAC (30 mL) and washed with pentane to afford 18 (7.42 g, $19.2 \mathrm{mmol}$, $73 \%$ ) as a white solid. ${ }^{1} \mathrm{H}$ NMR (400 MHz, DMSO) $\delta 7.96$ (m, $\left.2 \mathrm{H}, \mathrm{ArH}\right), 7.73$ (m, $\left.2 \mathrm{H}, \mathrm{ArH}\right)$. ${ }^{13} \mathrm{C}$ NMR (101 MHz, DMSO) $\delta 133.3,131.0,130.8,128.9,127.9,123.4(\mathrm{q}, J=290 \mathrm{~Hz})$, 117.2, 83.7 (m). IR 1464 (w), 1435 (w), 1290 (w), 1263 (m), 1185 (s), 1139 (s), 1103 (m), 1041 (w), 1021 (w), 952 (s), 760 (m), 730 (m), 692 (m).

\section{1-[(Triisopropylsilyl)ethynyl]-3,3-bis(trifluoromethyl)-3(1H)-1,2-benziodoxole (1a)}<smiles>OI1OC(C(F)(F)F)(C(F)(F)F)c2ccccc21</smiles>

18

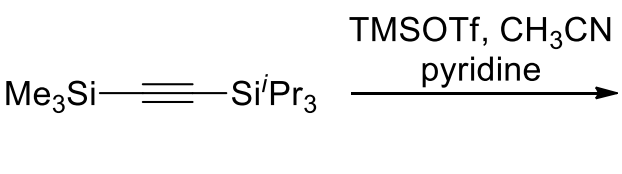

14<smiles>FC(F)(F)C1(C(F)(F)F)OI(C#C[SiH2])c2ccccc21</smiles>

$1 \mathrm{a}$

TMSOTf (3.80 g, $17.1 \mathrm{mmol}, 1.1$ equiv) was added to $\mathbf{1 8}$ ( $6.00 \mathrm{~g}, 15.5 \mathrm{mmol}, 1.0$ equiv) in $\mathrm{CH}_{2} \mathrm{Cl}_{2}(200 \mathrm{~mL})$ at $\mathrm{RT}$. After $20 \mathrm{~min}$, the solution was concentrated at $0{ }^{\circ} \mathrm{C}$ under reduced pressure. After evaporation of the solvent, the reaction flask was directly filled with Ar, to prevent decomposition of the hygroscopic triflate intermediate. Then the resulting yellow solid was dissolved in $\mathrm{CH}_{3} \mathrm{CN}$ (200 mL). (Trimethylsilyl)(triiso-propylsilyl)acetylene (14) (5.14 g, $20.2 \mathrm{mmol}, 1.3$ equiv) was added and after $20 \mathrm{~min}$ several drops of pyridine were added. The reaction was then concentrated under vacuum, dissolved in $\mathrm{Et}_{2} \mathrm{O}$ and filtered over

[3]. A. J. Blake, A. Novak, M. Davies, R. I. Robinson, S. Woodward, Synth. Commun. 2009, 39, 1065 
a silica plug (eluant $\mathrm{Et}_{2} \mathrm{O}$ ). The resulting solid was recrystallized_from pentane to afford $\mathbf{1 a}$ (5.43 g, $9.87 \mathrm{mmol}, 64 \%)$ as white crystals.

Rf (PET/Et $2 \mathrm{O} 95 / 5): 0.4$ Mp $131-132^{\circ} \mathrm{C} .{ }^{1} \mathrm{H}$ NMR (400 MHz, $\left.\mathrm{CDCl}_{3}\right)(\mathrm{ca} 0.10 \mathrm{mmol} / \mathrm{mL}) \delta$ 8.36 (dd, $1 \mathrm{H}, J=7.9,1.7 \mathrm{~Hz}, \operatorname{ArH}), 7.84(\mathrm{~d}, 1 \mathrm{H}, J=6.7 \mathrm{~Hz}, \operatorname{ArH}), 7.68$ (m, 2H, ArH), 1.15 $(\mathrm{m}, 21 \mathrm{H}, \mathrm{TIPS}) .{ }^{13} \mathrm{C}$ NMR $\left(101 \mathrm{MHz}, \mathrm{CDCl}_{3}\right) \delta 132.7,131.1,129.9,129.9(\mathrm{~m}), 128.2,123.6$ (q, $288 \mathrm{~Hz}), 112.1,110.8,81.4(\mathrm{~m}), 69.7,18.5,11.2$. Characterization data of 1a corresponded to the literature values. ${ }^{[4]}$

\section{1-Hydroxy-1,2-benziodoxol-3-(1H)-one (20)}<smiles>O=C(O)c1ccccc1I</smiles>

19

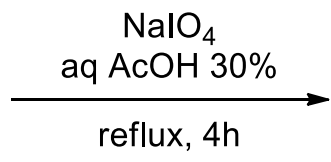

reflux, $4 \mathrm{~h}$<smiles>O=C1OI(O)c2ccccc21</smiles>

20

Following the reported procedure, ${ }^{[5]} \mathrm{NaIO}_{4}(7.24 \mathrm{~g}, 33.8 \mathrm{mmol}, 1.05$ equiv) and 2iodobenzoic acid (19) (8.00 g, $32.2 \mathrm{mmol}, 1.00$ equiv) were suspended in $30 \%$ (v:v) aq. $\mathrm{AcOH}(48 \mathrm{~mL})$. The mixture was vigorously stirred and refluxed for $4 \mathrm{~h}$. The reaction mixture was then diluted with cold water $(180 \mathrm{~mL})$ and allowed to cool to rt, protecting it from light. After $1 \mathrm{~h}$, the crude product was collected by filtration, washed on the filter with ice water ( 3 x $20 \mathrm{~mL})$ and acetone $(3 \times 20 \mathrm{~mL})$, and air-dried in the dark to give the pure product $20(8.3 \mathrm{~g}$, $31 \mathrm{mmol}, 98 \%$ ) as a colorless solid.

${ }^{1} \mathrm{H}$ NMR (400 MHz, $\left.\left(\mathrm{CD}_{3}\right)_{2} \mathrm{SO}\right) \delta 8.02(\mathrm{dd}, 1 \mathrm{H}, J=7.7,1.4 \mathrm{~Hz}, \operatorname{ArH}), 7.97(\mathrm{~m}, 1 \mathrm{H}, \mathrm{ArH})$, $7.85(\mathrm{dd}, 1 \mathrm{H}, J=8.2,0.7 \mathrm{~Hz}, \operatorname{Ar} H), 7.71(\mathrm{td}, 1 \mathrm{H}, J=7.6,1.2 \mathrm{~Hz}, \operatorname{Ar} H) ;{ }^{13} \mathrm{C} \mathrm{NMR}(100$ $\left.\mathrm{MHz},\left(\mathrm{CD}_{3}\right)_{2} \mathrm{SO}\right) \delta 167.7,134.5,131.5,131.1,130.4,126.3,120.4$; IR v $3083(\mathrm{w}), 3060(\mathrm{w})$, 2867 (w), 2402 (w), 1601 (m), 1585 (m), $1564(\mathrm{~m}), 1440$ (m), 1338 (s), 1302 (m), 1148 (m), $1018(\mathrm{w}), 834(\mathrm{~m}), 798(\mathrm{w}), 740(\mathrm{~s}), 694(\mathrm{~s}), 674(\mathrm{~m}), 649(\mathrm{~m})$; the reported values correspond to the ones in literature. ${ }^{[5]}$

\section{1-[(Triiso-propylsilyl)ethynyl]-1,2-benziodoxol-3(1H)-one (TIPS-EBX, 1b)}<smiles>O=C1OI(O)c2ccccc21</smiles>

20

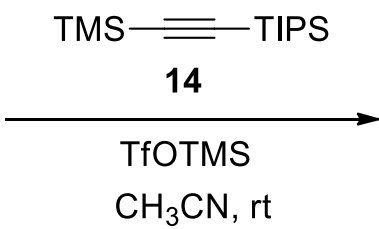

$\mathrm{CH}_{3} \mathrm{CN}$, rt<smiles>O=C1OI(C#CSC(F)(F)F)c2ccccc21</smiles>

$1 b$

Following a reported procedure ${ }^{[6]}$ 2-iodosylbenzoic acid (20) $(21.7 \mathrm{~g}, 82.0 \mathrm{mmol}, 1.0$ equiv) was charged in oven-dried three-neck 1L flask equipped with a magnetic stirrer. After 3 vacuum/nitrogen cycles, anhydrous acetonitrile $(500 \mathrm{~mL})$ was added via canula and cooled to $0{ }^{\circ} \mathrm{C}$. Trimethylsilyltriflate $(16.4 \mathrm{~mL}, 90.0 \mathrm{mmol}, 1.1$ equiv) was added dropwise via a dropping funnel over $30 \mathrm{~min}$ (no temperature increase was observed). After $15 \mathrm{~min}$,

[4] Y. LI, J. Brand, J. Waser, Angew. Chem., Int. Ed. 2013, 52, 6743.

[5]. L. Kraszkiewicz, L. Skulski, Arkivoc. 2003, 6, 120.

[6] J. P. Brand, J. Waser, Angew. Chem., Int. Ed. 2010, 49, 7304. 
(trimethylsilyl)(triisopropylsilyl)acetylene (14) (23.0 g, $90.0 \mathrm{mmol}, 1.1$ equiv) was added via canula over $15 \mathrm{~min}$ (no temperature increase was observed). After $30 \mathrm{~min}$, the suspension became an orange solution. After $10 \mathrm{~min}$, pyridine $(7.0 \mathrm{~mL}, 90 \mathrm{mmol}, 1.1$ equiv) was added via syringe. After $15 \mathrm{~min}$, the reaction mixture was transferred in a one-neck 1L flask and reduced under vacuum until a solid was obtained. The solid was dissolved in DCM $(200 \mathrm{~mL})$ and transferred in a 1L separatory funnel. The organic layer was added and washed with $1 \mathrm{M}$ $\mathrm{HCl}(200 \mathrm{~mL})$ and the aqueous layer was extracted with $\mathrm{CH}_{2} \mathrm{Cl}_{2}(200 \mathrm{~mL})$. The organic layers were combined, washed with a saturated solution of $\mathrm{NaHCO}_{3}(2 \times 200 \mathrm{~mL})$, dried over $\mathrm{MgSO}_{4}$, filtered and the solvent was evaporated under reduced pressure. Recrystallization from acetonitrile ( $c$ a $120 \mathrm{~mL})$ afforded $\mathbf{1 b}(30.1 \mathrm{~g}, 70.2 \mathrm{mmol}, 86 \%)$ as colorless crystals.

Melting point (Dec.) $170-176^{\circ} \mathrm{C}$

${ }^{1} \mathrm{H}$ NMR (400 MHz, CDCl3) $\delta 8.44(\mathrm{~m}, 1 \mathrm{H}, \mathrm{ArH}), 8.29$ (m, $\left.1 \mathrm{H}, \operatorname{Ar} H\right), 7.77$ (m, $\left.2 \mathrm{H}, \operatorname{ArH}\right)$, 1.16 (m, $21 \mathrm{H}$, TIPS). ${ }^{13} \mathrm{C}$ NMR (100 MHz, CDCl3) $\delta 166.4,134.6,132.3,131.4,131.4$, 126.1, 115.6, 114.1, 64.6, 18.4, 11.1. IR $v 2943(\mathrm{~m}), 2865(\mathrm{~m}), 1716(\mathrm{~m}), 1618(\mathrm{~m}), 1604(\mathrm{~s})$, $1584(\mathrm{~m}), 1557$ (m), 1465 (m), 1439 (w), 1349 (m), 1291 (m), 1270 (w), 1244 (m), 1140 (m), $1016(\mathrm{~m}), 999(\mathrm{~m}), 883(\mathrm{~m}), 833(\mathrm{~m}), 742(\mathrm{~m}), 702(\mathrm{~s}), 636(\mathrm{~m})$; Characterization data of $\mathbf{1 b}$ corresponded to the literature values. ${ }^{[6]}$

\section{Phenyl(triisopropylsilyl)iodonium triflate (1c)}<smiles>CC(=O)OI(OC(C)=O)c1ccccc1</smiles>

21

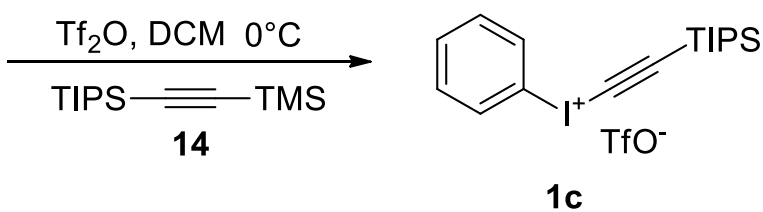

Following a slight modification of the reported procedure, ${ }^{[7]}$ phenyliodonium diacetate (21) (2.53 g, $7.85 \mathrm{mmol}, 1.00$ equiv) was diluted with DCM (7 mL) and the mixture was stirred for 5 minutes. $\mathrm{Tf}_{2} \mathrm{O}(0.60 \mathrm{~mL}, 3.9 \mathrm{mmol}, 0.50$ equiv. $)$ was added dropwise at $0{ }^{\circ} \mathrm{C}$ and the resulting yellow mixture was stirred $30 \mathrm{~min}$. (Trimethylsilyl)(triisopropylsilyl)acetylene (14) ( $2.00 \mathrm{~g}, 7.86 \mathrm{mmol}, 1.00$ equiv) was added and the mixture was then stirred $2 \mathrm{~h}$. Water was then added $(30 \mathrm{~mL})$ followed by extraction of the aqueous layer with DCM $(2 \times 30 \mathrm{~mL})$. The combined organic layers were dried over $\mathrm{MgSO}_{4}$, filtered and the solvent was evaporated under reduced pressure. The resulting solid was triturated in hexane $(10 \mathrm{~mL})$. Filtration and removal of solvent in vacuo afforded phenyl(triisopropylsilyl)iodonium triflate (1c) $(2.90 \mathrm{~g}$, $11.2 \mathrm{mmol}, 70 \%$ yield) as a colorless solid.

Melting point: $109-114^{\circ} \mathrm{C} .{ }^{1} \mathrm{H}$ NMR $\left(400 \mathrm{MHz}, \mathrm{CDCl}_{3}\right) \delta 8.09(\mathrm{~m}, 2 \mathrm{H}, \mathrm{ArH}), 7.65(\mathrm{~m}, 1 \mathrm{H}$, $\mathrm{ArH}), 7.52$ (m, $2 \mathrm{H}, \mathrm{ArH}), 1.15-1.01$ (m, $21 \mathrm{H}, \mathrm{TIPS}) ;{ }^{13} \mathrm{C} \mathrm{NMR}\left(100 \mathrm{MHz}, \mathrm{CDCl}_{3}\right) \delta 133.7$, 132.5, 132.4, 119.7, 117.6, 117.6, 44.9, 18.3, 11.1; IR v 3288 (w), 3088 (m), 2949 (m), 2894 (m), 2869 (w), $1563(\mathrm{~m}), 1467(\mathrm{w}), 1451(\mathrm{w}), 1388(\mathrm{w}), 1281$ (s), 1236 (s), 1221 (s), 1174 (s), 1068 (w), 1028 (s), 988 (m), 916 (m), 884 (m), 736 (s), 679 (m), 639 (s); HRMS (ESI) calcd

[7] T. Kitamura, M. Kotani, Y. Fujiwara, Synthesis 1998, 10, 1416. 
for $\mathrm{C}_{17} \mathrm{H}_{26} \mathrm{ISi}^{+}$(M-OTf) 385.0843; found 385.0812; the reported values corresponded to the ones in literature. ${ }^{[5]}$ 


\section{Synthesis of starting materials:}

\section{1-(3-Methoxypropyl)-1H-pyrrole-2-carbaldehyde (24)}<smiles>c1c[nH]c(CCc2ccco2)c1</smiles>

22<smiles>COCCCBr</smiles>

23

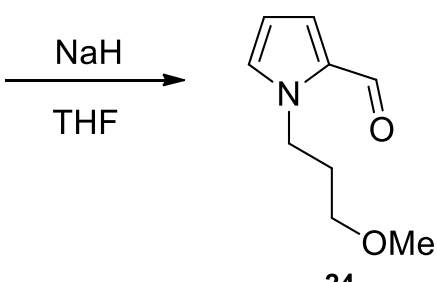

24

A solution of 22 (475 mg, $5.00 \mathrm{mmol}, 1.0$ equiv) in THF ( $5 \mathrm{~mL}$ ) was added into a suspension of $\mathrm{NaH}$ (126 mg, $5.25 \mathrm{mmol}, 1.05$ equiv) in THF ( $5 \mathrm{~mL}$ ). 15 minutes later, 23 (765 mg, 5.00 mmol, 1.00 equiv) was added into the mixture. The reaction was quenched by sat $\mathrm{NH}_{4} \mathrm{Cl}$ after $4 \mathrm{~h}$. The aqueous layer and organic layer were separated and the aqueous layer was extracted with ether $(3 \times 10 \mathrm{~mL})$. After drying over $\mathrm{MgSO}_{4}$, filtrating and concentrating under vacuum, the crude product was purified by column chromatography (Pentane/EtOAc=10:1) to afford 24 as brown oil (500 mg, $3.00 \mathrm{mmol}, 60 \%$ ).

Rf (Pentane / EtOAc= 10:1): 0.4. ${ }^{1} \mathrm{H}$ NMR (400 MHz, $\left.\mathrm{CDCl}_{3}\right) \delta 9.37$ (s, $\left.1 \mathrm{H}, \mathrm{CHO}\right), 6.81$ (s, $1 \mathrm{H}$, pyrroleH), 6.78 (dd, $1 \mathrm{H}, J=4.0,1.7 \mathrm{~Hz}$, pyrroleH), 6.06 (dd, $1 \mathrm{H}, J=4.0,2.5 \mathrm{~Hz}$, pyrroleH), $4.24\left(\mathrm{t}, 2 \mathrm{H}, J=6.8 \mathrm{~Hz}, N_{C H}\right), 3.16\left(\mathrm{~s}, 3 \mathrm{H}, O \mathrm{OCH}_{3}\right), 3.12(\mathrm{t}, 2 \mathrm{H}, J=5.8 \mathrm{~Hz}$, $\left.\mathrm{CH}_{2} \mathrm{OCH}_{3}\right), 1.85\left(\mathrm{~m}, 2 \mathrm{H}, \mathrm{CH}_{2}\right) .{ }^{13} \mathrm{C}$ NMR $\left(101 \mathrm{MHz}, \mathrm{CDCl}_{3}\right) \delta 178.5,131.4,130.8,124.3$, 108.9, 68.4, 57.9, 45.5, 30.5. IR 1659 (m), 1482 (w), 1405 (w), 1370 (w), 1322 (w), 1117 (w), 1071 (w), 913 (w), 732 (s). HRMS (ESI) calcd for $\mathrm{C}_{9} \mathrm{H}_{13} \mathrm{NNaO}_{2}{ }^{+}[\mathrm{M}+\mathrm{Na}]^{+}$190.0838; found 190.0834 .

\section{1-(4,4,4-Trifluorobutyl)-1H-pyrrole-2-carbaldehyde (26)}<smiles>O=Cc1ccc[nH]1</smiles>

22<smiles>FC(F)(F)CCCCBr</smiles>

25

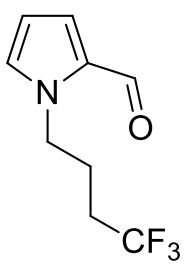

26

A solution of 22 (475 mg, $5.00 \mathrm{mmol}, 1.0$ equiv) in THF ( $5 \mathrm{~mL}$ ) was added into a solution of $\mathrm{NaH}$ (126 mg, $5.25 \mathrm{mmol}, 1.05$ equiv) in THF (5 mL). 15 minutes later, 25 (955 mg, 5.00 mmol, 1.00 equiv) was added into the mixture. The reaction was quenched with sat $\mathrm{NH}_{4} \mathrm{Cl}$ after $4 \mathrm{~h}$. The aqueous and organic layers were separated and the aqueous layer was extracted with ether $(3 \times 10 \mathrm{~mL})$. After drying over $\mathrm{MgSO}_{4}$, filtrating and concentrating under vacuum, the crude product was purified by column chromatography (Pentane/EtOAc $=20: 1)$ to afford 26 as yellow oil (720 mg, $3.51 \mathrm{mmol}, 70 \%)$.

Rf (Pentane / EtOAc= 20:1): 0.5. ${ }^{1} \mathrm{H}$ NMR (400 MHz, $\left.\mathrm{CDCl}_{3}\right) \delta 9.44(\mathrm{~d}, 1 \mathrm{H}, J=1.0 \mathrm{~Hz}$, CHO), 6.87 (m, $2 \mathrm{H}$, pyrroleH), $6.16(\mathrm{dd}, 1 \mathrm{H}, J=4.0,2.5 \mathrm{~Hz}$, pyrroleH), $4.30(\mathrm{t}, 2 \mathrm{H}, J=6.8$ $\left.\mathrm{Hz} \mathrm{NCH}_{2}\right), 1.97\left(\mathrm{~m}, 4 \mathrm{H}, \mathrm{CH}_{2} \mathrm{CH}_{2}\right) .{ }^{13} \mathrm{C} \mathrm{NMR}\left(101 \mathrm{MHz}, \mathrm{CDCl}_{3}\right) \delta 179.0,131.0,126.7$ (q, $J$ 
$=276.6 \mathrm{~Hz}$ ), 124.9, 109.6 (two peaks overlap), 47.1, 30.3 (q, $J=29.3 \mathrm{~Hz}$ ), 23.7 (q, $J=2.9$ Hz). IR 1660 (m), 1482 (w), 1409 (w), 1394 (w), 1370 (w), 1338 (w), 1327 (w), 1316 (w), $1297(\mathrm{w}), 1280$ (w), 1255 (m), 1240 (w), 1221 (w), $1184(\mathrm{w}), 1136$ (m), $1079(\mathrm{w}), 1065$ (w), $1033(\mathrm{~m}), 1018$ (w), 910 (m), 766 (m), 729 (s). HRMS (ESI) calcd for $\mathrm{C}_{9} \mathrm{~F}_{3} \mathrm{H}_{11} \mathrm{NO}^{+}[\mathrm{M}+\mathrm{H}]^{+}$ 206.0787; found 206.0786.

\section{1-Methyl-1H-pyrrole-3-carbaldehyde (29)}

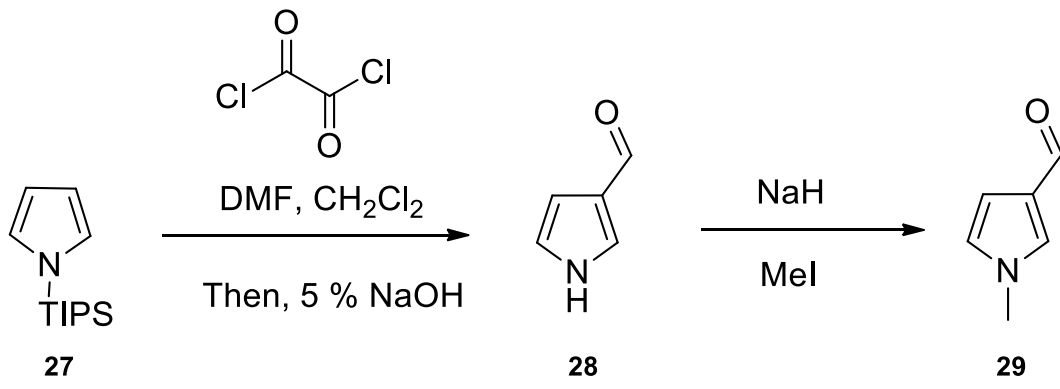

Following a reported procedure ${ }^{[8]}$, dry DMF $(1.88 \mathrm{~mL}, 24.3 \mathrm{mmol}, 1.2$ equiv) was added into a solution of oxalyl dichloride $(1.82 \mathrm{~mL}, 22.3 \mathrm{mmol}, 1.1$ equiv) in DCM (50 mL) slowly at 0 ${ }^{\circ} \mathrm{C}$. After 15 minutes, the solution turned cloudy. 27 (4.50 g, $20.2 \mathrm{mmol}, 1.0$ equiv) was added into the mixture slowly at $0{ }^{\circ} \mathrm{C}$, following by heating the solution at reflux during $1 \mathrm{~h}$. The solution was cooled down and filtrated. The filtrating cake was dissolved into $5 \% \mathrm{NaOH}$ solution $(200 \mathrm{~mL})$. The solution was kept stirring during 4 hours, and extracted by ether $(3 \mathrm{x}$ $50 \mathrm{~mL}$ ). After drying over $\mathrm{MgSO}_{4}$, filtrating and concentrating under vacuum, yellow oil 28 was obtained as a yellow oil $(735 \mathrm{mg}, 7.73 \mathrm{mmol}$, <38\%). The crude product was used without further purification.

A solution of $28(735 \mathrm{mg}, 7.73 \mathrm{mmol}, 1.0$ equiv) in THF ( $8 \mathrm{~mL})$ was added into a suspension of $\mathrm{NaH}(291 \mathrm{mg}, 8.50 \mathrm{mmol}, 1.1$ equiv) in THF ( $8 \mathrm{~mL})$. After 15 minutes, $\mathrm{CH}_{3} \mathrm{I}$ $(0.53 \mathrm{~mL}, 8.5 \mathrm{mmol}, 1.1$ equiv) was added into the solution. The reaction was quenched with sat $\mathrm{NH}_{4} \mathrm{Cl}$ after $4 \mathrm{~h}$. The aqueous and organic layers were separated and the aqueous layer was extracted with ether $(3 \times 10 \mathrm{~mL})$. After drying over $\mathrm{MgSO}_{4}$, filtrating and concentrating under vacuum, the crude product was purified by column chromatography (Pentane/EtOAc $=10: 1$ ) to afford 29 as brown oil $(610 \mathrm{mg}, 5.59 \mathrm{mmol}, 72 \%)$.

${ }^{1} \mathrm{H} \mathrm{NMR}\left(400 \mathrm{MHz}, \mathrm{CDCl}_{3}\right) \delta 9.66(\mathrm{~s}, 1 \mathrm{H}, \mathrm{CHO}), 7.20(\mathrm{t}, 1 \mathrm{H}, J=1.8 \mathrm{~Hz}$, pyrroleH), 6.59 (m, $1 \mathrm{H}$, pyrroleH), $6.55\left(\mathrm{dd}, 1 \mathrm{H}, J=2.9,1.8 \mathrm{~Hz}\right.$, pyrroleH), 3.66 (s, $\left.3 \mathrm{H}, \mathrm{CH}_{3}\right) .{ }^{13} \mathrm{C} \mathrm{NMR}$ $\left(101 \mathrm{MHz}, \mathrm{CDCl}_{3}\right) \delta 185.0,129.9,126.5,124.3,108.1,36.5$. Characterization data corresponded to the literature values ${ }^{[9]}$.

\section{2-(1-Methoxybut-3-yn-1-yl)-1-methyl-1H-pyrrole (6a)}

[8] B L. Bray, J M. Muchowaski, J. Org. Chem. 1988, 53, 6115.

[9] T Purkarthofer, K. Gruber, M. Fechter, H. Griengl,. Tetrahedron. 2005, 61, 7661. 


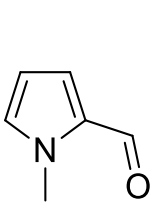

30

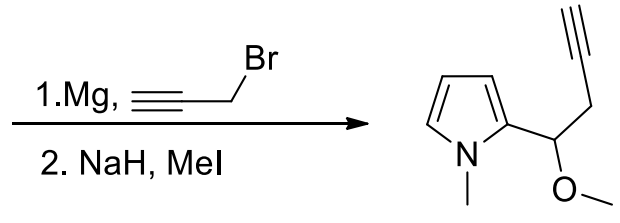

$6 a$

A $50 \mathrm{~mL}$ two-necked flask was charged with $\mathrm{Mg}$ (321 mg, $13.2 \mathrm{mmol}, 1.32$ equiv), $\mathrm{HgCl}_{2}$ ( $2.7 \mathrm{mg}, 0.10 \mathrm{mmol}, 0.01$ equiv) and dry diethyl ether $(30 \mathrm{~mL})$, then propargyl bromide was added dropwise ( $2.86 \mathrm{~g}, 12.0 \mathrm{mmol}, 1.20$ equiv). When the solution became homogeneous, 30 (1.09 g, $10.0 \mathrm{mmol}, 1.00$ equiv) was added dropwise. The reaction was quenched with sat $\mathrm{NH}_{4} \mathrm{Cl}$ solution $(30 \mathrm{~mL}$ ) when TLC (Pentane/EtOAc: 5/1) indicated that the aldehyde was completely consumed after 4 hours. The aqueous and organic layers were separated; the aqueous layer was extracted with diethyl ether $(3 \times 20 \mathrm{~mL})$. The combined organic layers were dried over $\mathrm{MgSO}_{4}$ and concentrated under vaccum to obtain $1.34 \mathrm{~g}$ crude product as brown oil. The crude product was used for the next step directly without further purification. Crude propargylic alcohol (1.34 g, $8.98 \mathrm{mmol}, 0.89$ equiv) in THF (18 mL) was added into a suspension of $\mathrm{NaH}$ (237 mg, $9.88 \mathrm{mmol}, 0.98$ equiv) in THF (18 mL). 15 minutes later, MeI ( $1.40 \mathrm{~g}, 9.88 \mathrm{mmol}, 1.10$ equiv) was added into the mixture. The reaction was quenched with sat $\mathrm{NH}_{4} \mathrm{Cl}$ after $4 \mathrm{~h}$. The aqueous and organic layers were separated and the aqueous layer was extracted with ether $(3 \times 10 \mathrm{~mL})$. After drying over $\mathrm{MgSO}_{4}$, filtrating and concentrating under vacuum, the crude product was purified by column chromatography (Pentane/EtOAc $=15: 1$, $\left.1 \% \mathrm{Et}_{3} \mathrm{~N}\right)$ to afford $\mathbf{6 a}$ as brown oil (1.00 g, $6.13 \mathrm{mmol}, 61 \%$ over two steps).

Rf (Pentane:EtOAc = $25 / 1): 0.6 .{ }^{1} \mathrm{H}$ NMR $\left(400 \mathrm{MHz}, \mathrm{CDCl}_{3}\right) \delta 6.62(\mathrm{~m}, 1 \mathrm{H}$, pyrroleH$)$, $6.18(\mathrm{dd}, 1 \mathrm{H}, J=3.6,1.8 \mathrm{~Hz}$, pyrroleH $), 6.10(\mathrm{dd}, 1 \mathrm{H}, J=3.6,2.7 \mathrm{~Hz}$, pyrroleH $), 4.58(\mathrm{t}, 1$ $\mathrm{H}, J=6.9 \mathrm{~Hz}, \mathrm{CHO}$ ), 3.68 (s, $\left.3 \mathrm{H}, \mathrm{NCH}_{3}\right), 3.27$ (s, $\left.3 \mathrm{H}, \mathrm{OCH}_{3}\right), 2.79$ (ddd, $2 \mathrm{H}, J=16.8,6.5$, $\left.2.7 \mathrm{~Hz}, \mathrm{CH}_{2}\right), 2.05(\mathrm{t}, 1 \mathrm{H}, \mathrm{J}=2.7 \mathrm{~Hz}$, alkyne $\mathrm{CH}) .{ }^{13} \mathrm{C} \mathrm{NMR}\left(101 \mathrm{MHz}, \mathrm{CDCl}_{3}\right) \delta 129.9$, 123.3, 108.5, 106.4, 80.7, 73.9, 69.8, 54.7, 34.0, 24.0. IR 2946 (m), 2866 (m), $2088(\mathrm{w}), 1707$ (s), $1502(\mathrm{w}), 1366(\mathrm{w}), 1274(\mathrm{w}), 1273(\mathrm{w}), 1272(\mathrm{w}), 1253(\mathrm{w}), 1205(\mathrm{~m}), 1175(\mathrm{w}), 998$ (w), $886(\mathrm{~m})$. HRMS (ESI) calcd for $\mathrm{C}_{10} \mathrm{H}_{13} \mathrm{NNaO}^{+}[\mathrm{M}+\mathrm{Na}]^{+}$186.0889; found 186.0895.

The protocol for the synthesis of $\mathbf{6 a}$ represents the typical procedure for the preparation of $\mathbf{6 b}$, 6c, 6d, 6e, 6f, 6j, 6k, 61, 6m, 9a, 9e, 9f, 9h, 9i, 9j.

\section{2-(1-Methoxybut-3-yn-1-yl)-1-(p-tolyl)-1H-pyrrole (6b)}

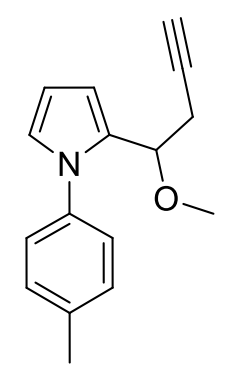

$6 \mathrm{~b}$

$1.0 \mathrm{~g}(5.4 \mathrm{mmol})$ of the corresponding aldehyde was used to obtain the crude propargylic alcohol (880 mg, $3.91 \mathrm{mmol}, 72 \%) .440 \mathrm{mg}(1.95 \mathrm{mmol})$ of crude propargylic alcohol was used for methylation. 2-(1-Methoxybut-3-yn-1-yl)-1(p-tolyl)-1H-pyrrole (6b) was obtained as a colorless oil $(398 \mathrm{mg}, 1.66 \mathrm{mmol}$, $62 \%$ over two steps).

Rf $($ Pentane / EtOAc = $15 / 1): 0.6 .{ }^{1} \mathrm{H}$ NMR $\left(400 \mathrm{MHz}, \mathrm{CDCl}_{3}\right) \delta 7.31(\mathrm{~m}, 4 \mathrm{H}$, benzene), 6.82 (dd, $1 \mathrm{H}, J=2.7,1.8 \mathrm{~Hz}$, pyrroleH), 6.43 (dd, $1 \mathrm{H}, J=3.6,1.8$ $\mathrm{Hz}$, pyrroleH), $6.32(\mathrm{~m}, 1 \mathrm{H}$, pyrroleH $), 4.44\left(\mathrm{t}, 1 \mathrm{H}, J=6.9 \mathrm{~Hz}, \mathrm{CHOCH}_{3}\right)$, $3.20\left(\mathrm{~s}, 3 \mathrm{H}, \mathrm{OCH}_{3}\right), 2.71$ (ddd, $\left.2 \mathrm{H}, J=15.2,7.0,2.7 \mathrm{~Hz}, \mathrm{CH}_{2}\right), 2.47$ (s, $3 \mathrm{H}$, tolyl $\mathrm{CH}_{3}$ ), 2.01 $(\mathrm{t}, 1 \mathrm{H}, J=2.7 \mathrm{~Hz}$, alkyne $C H) .{ }^{13} \mathrm{C}$ NMR $\left(101 \mathrm{MHz}, \mathrm{CDCl}_{3}\right) \delta 137.4,137.2,131.7,129.5$, 126.5, 123.4, 108.5, 108.0, 80.9, 73.0, 69.8, 55.3, 24.7, 21.0. IR 3303 (w), $2986(\mathrm{w}), 2928(\mathrm{w})$, 2902 (w), 2821 (w), 2246 (w), 2122 (w), 1518 (s), 1468 (m), 1432 (w), $1329(\mathrm{~m}), 1241(\mathrm{w})$, 
$1183(\mathrm{w}), 1150(\mathrm{w}), 1095(\mathrm{~s}), 1046(\mathrm{w}), 1034(\mathrm{w}), 974(\mathrm{w}), 941(\mathrm{w}), 912(\mathrm{~m}), 894(\mathrm{w}), 825(\mathrm{~s})$, $797(\mathrm{w}), 716$ (s), 687 (w), 672 (w), 645 (s), 636 (s), 625 (m), 613 (m). HRMS (ESI) calcd for $\mathrm{C}_{16} \mathrm{H}_{17} \mathrm{NaO}^{+}[\mathrm{M}+\mathrm{Na}]^{+}$262.1202; found 262.1198 .

\section{1-Benzyl-2-(1-methoxybut-3-yn-1-yl)-1H-pyrrole (6c)}

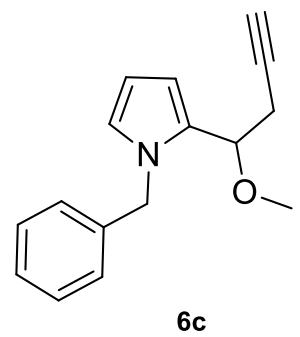

$690 \mathrm{mg}$ (3.73 mmol) of the corresponding aldehyde was used to obtain the crude propargylic alcohol (880 $\mathrm{mg}, 2.83 \mathrm{mmol}$, <76\%), which was used directly in the methylation step. 6c was obtained as a yellow oil $(678 \mathrm{mg}$, $2.83 \mathrm{mmol}, 76 \%$ over two steps).

Rf (Pentane / EtOAc = $25 / 1): 0.5 .{ }^{1} \mathrm{H}$ NMR $\left(400 \mathrm{MHz}, \mathrm{CDCl}_{3}\right) \delta 7.32(\mathrm{~m}$, $3 \mathrm{H}$, benzene), $7.09(\mathrm{~d}, 2 \mathrm{H}, J=7.0 \mathrm{~Hz}$, benzene), $6.72(\mathrm{dd}, 1 \mathrm{H}, J=2.6$, $1.7 \mathrm{~Hz}$, pyrroleH), $6.29(\mathrm{dd}, 1 \mathrm{H}, J=3.6,1.7 \mathrm{~Hz}$, pyrroleH), $6.21(\mathrm{~m}, 1 \mathrm{H}$, pyrroleH), $5.23\left(\mathrm{~s}, 2 \mathrm{H}, \mathrm{NCH}_{2}\right), 4.51\left(\mathrm{t}, 1 \mathrm{H}, J=6.8 \mathrm{~Hz}, \mathrm{CHOCH}_{3}\right), 3.17\left(\mathrm{~s}, 3 \mathrm{H}, \mathrm{OCH}_{3}\right), 2.69$ (ddd, $\left.2 \mathrm{H}, J=16.5,7.2,2.7 \mathrm{~Hz}, \mathrm{CH}_{2}\right), 2.02(\mathrm{t}, 1 \mathrm{H}, J=2.7 \mathrm{~Hz}$, alkyne $\mathrm{CH}) .{ }^{13} \mathrm{C}$ NMR $(101$ $\left.\mathrm{MHz}, \mathrm{CDCl}_{3}\right) \delta 138.0,130.1,128.3,127.0,126.2,122.8,108.5,107.0,80.6,73.5,69.9,54.5$, 50.2, 24.1. IR 2247 (w), 2123 (w), 1735 (w), $1497(w), 1482(w), 1455(w), 1357$ (w), 1299 (w), 1096 (m), 1078 (m), 909 (s), 728 (s). HRMS (ESI) calcd for $\mathrm{C}_{16} \mathrm{H}_{17} \mathrm{NNaO}^{+}[\mathrm{M}+\mathrm{Na}]^{+}$ 262.1202; found 262.1207.

\section{2-(1-Methoxybut-3-yn-1-yl)-1-(3-methoxypropyl)-1H-pyrrole (6d)}

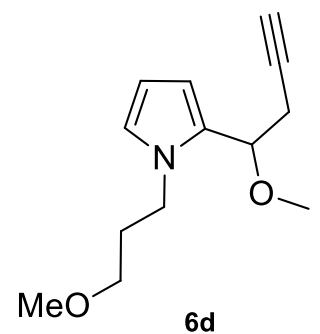

$573 \mathrm{mg}$ of 24 (3.73 mmol) was used to obtain the crude propargylic alcohol (490 mg, $2.21 \mathrm{mmol}$, <74\%), which was used directly in the methylation step. 6d was obtained as yellow oil (248 mg, $1.12 \mathrm{mmol}, 40 \%$ over two steps).

Rf $($ Pentane / EtOAc = $25 / 1): 0.6 .{ }^{1} \mathrm{H}$ NMR $\left(400 \mathrm{MHz}, \mathrm{CDCl}_{3}\right) \delta 6.66$ (dd, $1 \mathrm{H}, J=2.8,1.8 \mathrm{~Hz}$, pyrroleH), $6.15(\mathrm{~m}, 1 \mathrm{H}$, pyrroleH), 6.09 (dd, 1 $\mathrm{H}, J=3.5,2.8 \mathrm{~Hz}$, pyrroleH), $4.58\left(\mathrm{t}, 1 \mathrm{H}, J=6.9 \mathrm{~Hz}, \mathrm{CHOCH}_{3}\right), 4.03$ $\left(\mathrm{m}, 2 \mathrm{H}, \mathrm{NCH}_{2}\right), 3.33\left(\mathrm{~s}, 3 \mathrm{H}, \mathrm{CHOCH}_{3}\right), 3.32\left(\mathrm{~m}, 2 \mathrm{H}, \mathrm{CH}_{2} \mathrm{OCH}_{3}\right), 3.23\left(\mathrm{~s}, 3 \mathrm{H}, \mathrm{CH}_{2} \mathrm{OCH}_{3}\right)$, $2.77\left(\mathrm{ddd}, 2 \mathrm{H}, \mathrm{J}=16.2,6.7,2.7 \mathrm{~Hz}, \mathrm{CH}_{2}\right), 1.99\left(\mathrm{~m}, 3 \mathrm{H}\right.$, alkyne $\left.\mathrm{CH}+\mathrm{NCH}_{2} \mathrm{CH}_{2}\right) .{ }^{13} \mathrm{C} \mathrm{NMR}$ $\left(101 \mathrm{MHz}, \mathrm{CDCl}_{3}\right) \delta 129.8,122.0,107.8,106.8,80.8,73.1,69.9,68.9,58.3,54.4,43.3,31.3$, 23.9. IR 1664 (w), 1483 (w), 1458 (w), 1348 (w), 1297 (w), 1190 (w), $1116(\mathrm{~s}), 1075$ (s), 1029 (w), 914 (w), 833 (w), 717 (s). HRMS (ESI) calcd for $\mathrm{C}_{13} \mathrm{H}_{19} \mathrm{NaO}_{2}{ }^{+}[\mathrm{M}+\mathrm{Na}]^{+} 244.1308$; found 244.1306 .

\section{2-(1-Methoxybut-3-yn-1-yl)-1-(4,4,4-trifluorobutyl)-1H-pyrrole (6e)}

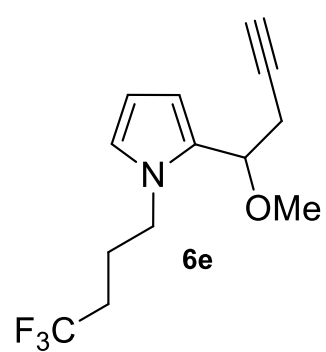

$720 \mathrm{mg}$ of 27 (3.51 mmol) was used to obtain the crude propargylic alcohol ( $850 \mathrm{mg}, 3.47 \mathrm{mmol}$, <99\%), which was used directly in the methylation step. 6e was obtained as yellow oil $(548 \mathrm{mg}, 2.11 \mathrm{mmol}$, $60 \%$ over two steps).

Rf (Pentane / EtOAc = $25 / 1): 0.4 .{ }^{1} \mathrm{H}$ NMR $\left(400 \mathrm{MHz}, \mathrm{CDCl}_{3}\right) \delta 6.67$ $(\mathrm{dd}, 1 \mathrm{H}, J=2.7,1.8 \mathrm{~Hz}$, pyrroleH), $6.21(\mathrm{dd}, 1 \mathrm{H}, J=3.6,1.8 \mathrm{~Hz}$, pyrroleH), $6.15(\mathrm{~m}, 1 \mathrm{H}$, pyrroleH $), 4.58\left(\mathrm{t}, 1 \mathrm{H}, J=7.0 \mathrm{~Hz}, \mathrm{CHOCH}_{3}\right)$, $4.03\left(\mathrm{~m}, 2 \mathrm{H}, \mathrm{NCH}_{2}\right.$ ), 3.25 (s, $3 \mathrm{OCH}_{3}$ ), 2.81 (ddd, $2 \mathrm{H}, J=16.8 \mathrm{~Hz}$, $\left.6.9 \mathrm{~Hz}, 2.7 \mathrm{~Hz}, \mathrm{CH}_{2}\right), 2.08\left(\mathrm{~m}, 4 \mathrm{H}, \mathrm{CH}_{2} \mathrm{CH}_{2} \mathrm{CF}_{3}\right), 2.03(\mathrm{t}, 1 \mathrm{H}, \mathrm{J}=2.7 \mathrm{~Hz}$, alkyne $\mathrm{CH}) .{ }^{13} \mathrm{C}$ NMR $\left(101 \mathrm{MHz}, \mathrm{CDCl}_{3}\right) \delta 129.8,126.8(\mathrm{q}, J=276.6 \mathrm{~Hz}), 121.8,108.7,107.4,80.5,73.3$, 70.1, 54.2, 45.3, 30.9 (q, $J=29.3 \mathrm{~Hz}), 23.9$ (q, $J=2.9$ Hz). 23.6. IR $3310(\mathrm{w}), 2945$ (w), 2338 (w), $1485(\mathrm{w}), 1455$ (w), 1393 (w), 1374 (w), 1318 (w), 1295 (m), 1255 (s), $1212(\mathrm{w}), 1135$ (s), 1119 (m), 1095 (m), 1080 (m), 1027 (m), 1009 (w), 913 (w), 833 (w), 795 (w), 720 (s). HRMS (ESI) calcd for $\mathrm{C}_{13} \mathrm{H}_{16} \mathrm{~F}_{3} \mathrm{NNaO}^{+}[\mathrm{M}+\mathrm{Na}]^{+} 282.1076$; found 282.1078. 


\section{2-(1-Methoxypent-3-yn-1-yl)-1-methyl-1H-pyrrole (6f)}

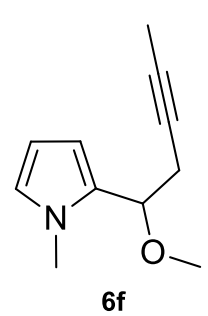

$1.0 \mathrm{~g}$ of 30 (5.4 mmol) was used to obtain crude propargylic alcohol $(880 \mathrm{mg}$, $3.91 \mathrm{mmol},<72 \%) .448 \mathrm{mg}$ of crude propargylic alcohol $(3.00 \mathrm{mmol}), \mathrm{NaH}$ (144 mg, $6.00 \mathrm{mmol}, 2$ equiv) and $\mathrm{CH}_{3} \mathrm{I}$ (1.06 g, $7.50 \mathrm{mmol}, 2.5$ equiv) were used for the methylation. $6 \mathbf{f}$ was obtained as yellow oil (384 mg, $1.83 \mathrm{mmol}, 48 \%$ over two steps).

Rf (Pentane / EtOAc = $15 / 1): 0.5 .{ }^{1} \mathrm{H} \mathrm{NMR}\left(400 \mathrm{MHz}, \mathrm{CDCl}_{3}\right) \delta 6.59(\mathrm{~m}, 1 \mathrm{H}$, pyrroleH), $6.15(\mathrm{dd}, 1 \mathrm{H}, J=3.6,1.8 \mathrm{~Hz}$, pyrrole $\mathrm{H}), 6.08(\mathrm{dd}, 1 \mathrm{H}, J=3.6,2.7$ $\mathrm{Hz}$, pyrroleH), $4.50\left(\mathrm{~m}, 1 \mathrm{H}, \mathrm{CHOCH}_{3}\right), 3.66\left(\mathrm{~s}, 3 \mathrm{H}, \mathrm{NCH}_{3}\right), 3.25\left(\mathrm{~s}, 3 \mathrm{H}, \mathrm{OCH}_{3}\right), 2.72(\mathrm{~m}, 2$ $\left.\mathrm{H}, \mathrm{CH}_{2}\right), 1.79$ (t, $\left.3 \mathrm{H}, J=2.6 \mathrm{~Hz}, \mathrm{CH}_{3}\right) .{ }^{13} \mathrm{C} \mathrm{NMR}\left(101 \mathrm{MHz}, \mathrm{CDCl}_{3}\right) \delta 130.4,123.1,108.2$, 106.4, 76.9, 75.4, 74.4, 54.6, 33.9, 24.3, 3.3. IR $2978(\mathrm{w}), 2937(\mathrm{w}), 2921(\mathrm{w}), 2869(\mathrm{w})$, $2821(\mathrm{w}), 1706(\mathrm{w}), 1494(\mathrm{w}), 1466(\mathrm{w}), 1465(\mathrm{w}), 1438(\mathrm{w}), 1421(\mathrm{w}), 1345(\mathrm{w}), 1302(\mathrm{~m})$, $1095(\mathrm{~s}), 1059(\mathrm{w}), 1003(\mathrm{w}), 955(\mathrm{w})$. HRMS (ESI) calcd for $\mathrm{C}_{11} \mathrm{H}_{15} \mathrm{NNaO}^{+}[\mathrm{M}+\mathrm{Na}]^{+}$ 200.1046; found 200.1047.

\section{2-(1-Methoxy-4-phenylbut-3-yn-1-yl)-1-methyl-1H-pyrrole (6g)}<smiles>C#CCC(OC)c1cccn1C</smiles>

$6 \mathbf{a}$<smiles>Ic1ccccc1</smiles>

31<smiles>COC(CC#Cc1ccccc1)c1cccn1C</smiles>

$6 \mathrm{~g}$

Iodobenzene (31) (179 mg, $0.880 \mathrm{mmol}, 1.1$ equiv) was added into a solution of $\mathbf{6 a}$ (130 $\mathrm{mg}$, $0.800 \mathrm{mmol}, 1.0$ equiv), $\mathrm{Pd}\left(\mathrm{PPh}_{3}\right) \mathrm{Cl}_{2}(6.3 \mathrm{mg}, 0.033 \mathrm{mmol}, 0.03$ equiv) and $\mathrm{CuI}(39 \mathrm{mg}$, $0.055 \mathrm{mmol}, 0.05$ equiv) in degased THF $(2 \mathrm{~mL})$ and $\mathrm{Et}_{3} \mathrm{~N}(2 \mathrm{~mL})$. The mixture was kept overnight at $60{ }^{\circ} \mathrm{C}$. The reaction was filtered through a plug of celite and the crude was purified by column chromatography (Pentane/EtOAc $=50: 1,1 \% \mathrm{Et}_{3} \mathrm{~N}$ ) to afford $\mathbf{6 g}$ as brown oil (236 mg, $0.986 \mathrm{mmol}, 90 \%$ ).

Rf (Pentane / EtOAc = 50 / 1): 0.3. ${ }^{1} \mathrm{H}$ NMR $\left(400 \mathrm{MHz}, \mathrm{CDCl}_{3}\right) \delta 7.41(\mathrm{~m}, 2 \mathrm{H}$, benzeneH $)$, $7.29(\mathrm{~m}, 3 \mathrm{H}$, benzeneH), $6.63(\mathrm{dd}, 1 \mathrm{H}, J=2.5,2.0 \mathrm{~Hz}$, pyrrole $\mathrm{H}), 6.24(\mathrm{~m}, 1 \mathrm{H}$, pyrroleH), $6.12\left(\mathrm{dd}, 1 \mathrm{H}, J=3.5,2.7 \mathrm{~Hz}\right.$, pyrroleH), $4.64\left(\mathrm{t}, 1 \mathrm{H}, J=6.9 \mathrm{~Hz}, \mathrm{CHOCH}_{3}\right), 3.70(\mathrm{~s}, 3 \mathrm{H}$, $\left.\mathrm{NCH}_{3}\right), 3.33\left(\mathrm{~s}, 3 \mathrm{H}, \mathrm{OCH}_{3}\right), 3.01\left(\mathrm{dd}, 2 \mathrm{H}, \mathrm{J}=16.8,6.6 \mathrm{~Hz}, \mathrm{CH}_{2}\right) .{ }^{13} \mathrm{C}$ NMR $(101 \mathrm{MHz}$, $\left.\mathrm{CDCl}_{3}\right) \delta 131.6,130.5,128.1,127.7,123.6,123.4,108.6,106.6,86.5,82.1,74.6,55.3,34.2$, 25.5. IR $2361(\mathrm{w}), 1491(\mathrm{~m}), 1465(\mathrm{w}), 1443(\mathrm{w}), 1417(\mathrm{w}), 1344(\mathrm{w}), 1300(\mathrm{w}), 1091(\mathrm{~s})$, 1061 (w), 950 (w), 910 (s), 758 (m), 729 (s). HRMS (ESI) calcd for $\mathrm{C}_{16} \mathrm{H}_{18} \mathrm{NO}^{+}[\mathrm{M}+\mathrm{H}]^{+}$ 240.1383 ; found 240.1388 .

The protocol for $\mathbf{6 g}$ represents the typical procedure for the synthesis of $6 \mathbf{h}, \mathbf{6 i}, \mathbf{9 c}$ and $9 \mathbf{d}$.

2-(1-Methoxy-4-(p-tolyl)but-3-yn-1-yl)-1-methyl-1H-pyrrole (6h) 
<smiles>C#CCC(OC)c1cccn1C</smiles>

$6 a$<smiles>Cc1ccc(I)cc1</smiles>

32<smiles>COC(CC#Cc1ccc(C)cc1)c1cccn1C</smiles>

$6 \mathrm{~h}$

Following the conditions described for $\mathbf{6 g}$, 6a (194 mg, $1.19 \mathrm{mmol})$ was used. The crude product was purified by flash column chromatography (Pentane/EtOAc $\left.=50: 1,1 \% \mathrm{Et}_{3} \mathrm{~N}\right)$ to afford $\mathbf{6 h}$ as yellow oil (267 $\mathrm{mg}, 1.05 \mathrm{mmol}, 88 \%$ )

Rf (Pentane / EtOAc = $50 / 1): 0.5 .{ }^{1} \mathrm{H}$ NMR $\left(400 \mathrm{MHz}, \mathrm{CDCl}_{3}\right) \delta 7.32(\mathrm{~d}, 2 \mathrm{H}, J=8.1 \mathrm{~Hz}$, benzeneH), $7.12(\mathrm{~m}, 2 \mathrm{H}$, benzeneH), $6.64(\mathrm{t}, 1 \mathrm{H}, J=2.0 \mathrm{~Hz}$, pyrroleH $), 6.25(\mathrm{dd}, 1 \mathrm{H}, J=$ 3.6, $1.8 \mathrm{~Hz}$, pyrroleH), $6.14(\mathrm{~m}, 1 \mathrm{H}$, pyrroleH $), 4.65\left(\mathrm{t}, 1 \mathrm{H}, J=6.9 \mathrm{~Hz}, \mathrm{CHOCH}_{3}\right), 3.71(\mathrm{~s}, 3$ $\left.\mathrm{H}, \mathrm{NCH}_{3}\right), 3.34\left(\mathrm{~s}, 3 \mathrm{H}, \mathrm{OCH}_{3}\right), 3.02\left(\mathrm{dd}, 2 \mathrm{H}, \mathrm{J}=16.8,6.6 \mathrm{~Hz}, \mathrm{CH}_{2}\right), 2.37\left(\mathrm{~s}, 3 \mathrm{H}, \mathrm{CH}_{3}\right) .{ }^{13} \mathrm{C}$ NMR $\left(101 \mathrm{MHz}, \mathrm{CDCl}_{3}\right) \delta 137.6,131.4,130.6,128.8,123.3,120.5,108.5,106.6,85.6,82.1$, 74.6, 55.2, 34.1, 25.5, 21.3. IR $2981(\mathrm{w}), 2927(\mathrm{~m}), 2362(\mathrm{w}), 1712(\mathrm{w}), 1508(\mathrm{~m}), 1496(\mathrm{w})$, 1445 (w), 1444 (w), 1345 (w), $1301(\mathrm{w}), 1267(\mathrm{w}), 1186(\mathrm{w}), 1093$ (s), $950(\mathrm{w}), 820$ (s), 786 (w), 717 (s). HRMS (ESI) calcd for $\mathrm{C}_{17} \mathrm{H}_{20} \mathrm{NO}^{+}[\mathrm{M}+\mathrm{H}]^{+} 254.1539$; found 254.1542.

\section{2-(4-(4-Bromophenyl)-1-methoxybut-3-yn-1-yl)-1-methyl-1H-pyrrole (6i)}<smiles>C#CCC(OC)c1cccn1C</smiles>

$6 a$<smiles>Brc1ccc(I)cc1</smiles>

33

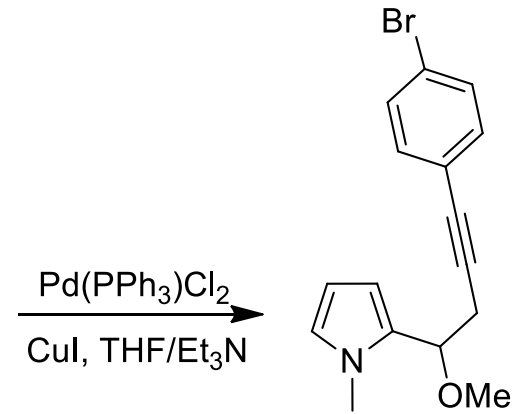

$6 \mathbf{i}$

Following the conditions described for $\mathbf{6 g}$, 6a $(163 \mathrm{mg}, 1.00 \mathrm{mmol})$ was used. The crude product was purified by flash column chromatography (Pentane/EtOAc $\left.=20: 1,1 \% \mathrm{Et}_{3} \mathrm{~N}\right)$ to afford 6i as yellow oil (260 $\mathrm{mg}, 0.817 \mathrm{mmol}, 82 \%$ )

$\operatorname{Rf}($ Pentane / EtOAc = $20 / 1): 0.2$

${ }^{1} \mathrm{H}$ NMR (400 MHz, $\left.\mathrm{CDCl}_{3}\right) \delta 7.31(\mathrm{~d}, 2 \mathrm{H}, J=8.5 \mathrm{~Hz}$, benzeneH), $7.15(\mathrm{~d}, 2 \mathrm{H}, J=8.5 \mathrm{~Hz}$ benzeneH), $6.52(\mathrm{t}, 1 \mathrm{H}, J=2.1 \mathrm{~Hz}$, pyrrole $H), 6.12(\mathrm{dd}, 1 \mathrm{H}, J=3.5,1.7 \mathrm{~Hz}$, pyrroleH), 6.01 $\left(\mathrm{m}, 1 \mathrm{H}\right.$, pyrroleH), $4.53\left(\mathrm{t}, 1 \mathrm{H}, J=6.9 \mathrm{~Hz}, \mathrm{CHOCH}_{3}\right), 3.59\left(\mathrm{~s}, 3 \mathrm{H}, \mathrm{NCH}_{3}\right), 3.21(\mathrm{~s}, 3 \mathrm{H}$, $\left.\mathrm{OCH}_{3}\right), 2.89\left(\mathrm{ddd}, 2 \mathrm{H}, \mathrm{J}=16.9,7.2,2.6 \mathrm{~Hz}, \mathrm{CH}_{2}\right) .{ }^{13} \mathrm{C} \mathrm{NMR}\left(101 \mathrm{MHz}, \mathrm{CDCl}_{3}\right) \delta 133.0$, $131.3,130.3,123.4,122.5,121.7,108.6,106.6,87.8,81.0,74.3,55.2,34.2,25.4$. IR $2981(\mathrm{~m})$, 2930 (m), 2907 (m), 2819 (w), 1488 (m), 1344 (w), 1300 (w), 1076 (s), 1008 (w), 990 (w), 
826 (s), 787 (w), 716 (s). HRMS (ESI) calcd for $\mathrm{C}_{16} \mathrm{H}_{17} \mathrm{BrNO}^{+}[\mathrm{M}+\mathrm{H}]^{+}$318.0488; found 318.0484 .

\section{2-(1-Methoxy-4-(naphthalen-1-yl)but-3-yn-1-yl)-1-methyl-1H-pyrrole (6j)}<smiles>C#CCC(OC)c1cccn1C</smiles>

$6 a$<smiles>Ic1cccc2ccccc12</smiles>

34

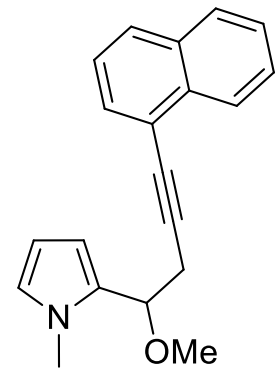

6j

Following the conditions described for $\mathbf{6 g}$, 6a $(163 \mathrm{mg}, 1.00 \mathrm{mmol})$ was used. The crude product was purified by flash column chromatography (Pentane/EtOAc $\left.=50: 1,1 \% \mathrm{Et}_{3} \mathrm{~N}\right)$ to afford $\mathbf{6 j}$ as yellow oil (269 $\mathrm{mg}, 0.930 \mathrm{mmol}, 93 \%$ )

Rf (Pentane / EtOAc = $50 / 1): 0.3 .{ }^{1} \mathrm{H}$ NMR $\left(400 \mathrm{MHz}, \mathrm{CDCl}_{3}\right) \delta 8.24(\mathrm{~d}, 1 \mathrm{H}, J=8.0 \mathrm{~Hz}$, benzeneH), $7.84(\mathrm{~m}, 2 \mathrm{H}$, benzeneH $), 7.67(\mathrm{~d}, 1 \mathrm{H}, J=6.8 \mathrm{~Hz}$, benzeneH), $7.56(\mathrm{~m}, 2 \mathrm{H}$, benzeneH), $7.43(\mathrm{~m}, 1 \mathrm{H}$, benzene $H), 6.69(\mathrm{~m}, 1 \mathrm{H}$, pyrrole $H), 6.37(\mathrm{dd}, 1 \mathrm{H}, J=3.5,1.7 \mathrm{~Hz}$, pyrroleH), $6.22(\mathrm{~m}, 1 \mathrm{H}$, pyrroleH $), 4.76\left(\mathrm{t}, 1 \mathrm{H}, J=7.1 \mathrm{~Hz}, \mathrm{CHOCH}_{3}\right), 3.74\left(\mathrm{~m}, 3 \mathrm{H}, \mathrm{NCH}_{3}\right)$, $3.39\left(\mathrm{~s}, 3 \mathrm{H}, \mathrm{OCH}_{3}\right), 3.21\left(\mathrm{dd}, 2 \mathrm{H}, J=16.8,6.6 \mathrm{~Hz}, \mathrm{CH}_{2}\right) .{ }^{13} \mathrm{C} \mathrm{NMR}\left(101 \mathrm{MHz}, \mathrm{CDCl}_{3}\right) \delta$ $133.4,133.0,130.4,129.9,128.0,128.0,126.5,126.3,126.1,125.0,123.4,121.2,108.9$, 106.7, 91.4, 80.2, 74.6, 55.3, 34.2, 25.8. IR 3053 (w), $2979(\mathrm{w}), 2931(\mathrm{~m}), 1738(\mathrm{w}), 1664(\mathrm{w})$, $1582(\mathrm{w}), 1497(\mathrm{~m}), 1466(\mathrm{w}), 1427(\mathrm{w}), 1405$ (w), 1343 (w), 1300 (w), $1198(\mathrm{w}), 1187(\mathrm{w})$, 1168 (w), 1162 (w), 1092 (s), 969 (w), 802 (s), 778 (s), 718 (s). HRMS (ESI) calcd for $\mathrm{C}_{20} \mathrm{H}_{20} \mathrm{NO}^{+}[\mathrm{M}+\mathrm{H}]^{+}$290.1539; found 290.1541.

\section{2-(1-Methoxy-2-methylbut-3-yn-1-yl)-1-methyl-1H-pyrrole (6k)}

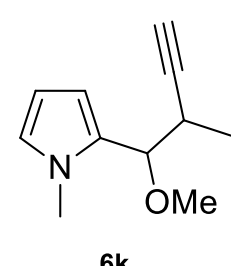

6k

$218 \mathrm{mg}$ of $\mathbf{3 0}$ (2.00 mmol, 1.0 equiv) and 3-bromobut-1-yne (319 mg, 2.40 mmol) were used to obtain the crude propargylic alcohol $(300 \mathrm{mg}, 1.84 \mathrm{mmol}$, $<92 \%$ ), which was used directly in the methylation step. 6k was obtained as transparent oil $(260 \mathrm{mg}, 1.47 \mathrm{mmol}, 74 \%$ over two steps as a mixture of diastereoisomers, the major one was characterized).

Rf (Pentane / EtOAc = $15 / 1): 0.5 .{ }^{1} \mathrm{H}$ NMR $\left(400 \mathrm{MHz}, \mathrm{CDCl}_{3}\right) \delta 6.58(\mathrm{~m}, 1 \mathrm{H}$, pyrroleH $)$, $6.11\left(\mathrm{~m}, 1 \mathrm{H}\right.$, pyrroleH), 6.07 (m, $1 \mathrm{H}$, pyrroleH), $4.22\left(\mathrm{~d}, 1 \mathrm{H}, J=8.9 \mathrm{~Hz}, \mathrm{CHOCH}_{3}\right), 3.66$ (s, $\left.3 \mathrm{H}, \mathrm{NCH}_{3}\right), 3.24\left(\mathrm{~s}, 3 \mathrm{H}, \mathrm{OCH}_{3}\right), 2.93\left(\mathrm{~m}, 1 \mathrm{H}, \mathrm{CHCH}_{3}\right), 2.15(\mathrm{~d}, 1 \mathrm{H}, J=2.4 \mathrm{~Hz}$, alkyne $\mathrm{CH}), 1.05\left(\mathrm{~d}, 3 \mathrm{H}, J=7.1 \mathrm{~Hz}, \mathrm{CH}_{3}\right) .{ }^{13} \mathrm{C} \mathrm{NMR}\left(101 \mathrm{MHz}, \mathrm{CDCl}_{3}\right) \delta 128.8,123.6,110.3$, 106.7, 86.7, 80.2, 69.2, 56.1, 34.5, 31.4, 17.7. IR 3291 (w), 3282 (w), 2979 (w), 2935 (w), $2901(\mathrm{w}), 2821(\mathrm{w}), 1493(\mathrm{w}), 1451(\mathrm{w}), 1417(\mathrm{w}), 1376(\mathrm{w}), 1298(\mathrm{~m}), 1204(\mathrm{w}), 1132(\mathrm{w})$, 1092 (s), 960 (w), 924 (w), $805(\mathrm{w}), 783$ (w), 715 (s), $688(\mathrm{~m}), 678(\mathrm{~m}), 651(\mathrm{~s}), 632(\mathrm{~m}), 615$ (m). HRMS (ESI) calcd for $\mathrm{C}_{11} \mathrm{H}_{16} \mathrm{NO}^{+}[\mathrm{M}+\mathrm{H}]^{+}$178.1226; found 178.1235.

\section{2-(2-Methoxypent-4-yn-2-yl)-1-methyl-1H-pyrrole (6l)}

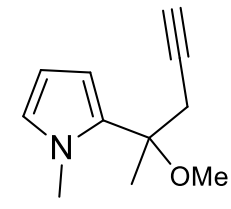

6I

$246 \mathrm{mg}$ of the corresponding ketone $(2.00 \mathrm{mmol})$ was used to obtain the crude propargylic alcohol (300 $\mathrm{mg}, 1.84 \mathrm{mmol}$, <92\%), which was used directly in 
the methylation step. 61 was obtained as yellow oil (230 mg, $1.30 \mathrm{mmol}, 65 \%$ over two steps). Rf (Pentane / EtOAc = $15 / 1): 0.6 .{ }^{1} \mathrm{H}$ NMR $\left(400 \mathrm{MHz}, \mathrm{CDCl}_{3}\right) \delta 6.59(\mathrm{~m}, 1 \mathrm{H}$, pyrroleH$)$, 6.07 (dd, $1 \mathrm{H}, J=3.7,1.9 \mathrm{~Hz}$, pyrroleH), 6.03 (dd, $1 \mathrm{H}, J=3.7,2.7 \mathrm{~Hz}$, pyrroleH), 3.76 (s, 3 $\left.\mathrm{H}, \mathrm{NCH}_{3}\right), 3.07\left(\mathrm{~s}, 3 \mathrm{H}, \mathrm{OCH}_{3}\right), 2.82\left(\mathrm{dd}, 2 \mathrm{H}, \mathrm{J}=16.0 \mathrm{~Hz}, 2.7 \mathrm{~Hz}, \mathrm{CH}_{2}\right), 2.05(\mathrm{t}, 1 \mathrm{H}, \mathrm{J}=2.7$ $\mathrm{Hz}$, alkyne $\mathrm{CH}), 1.70\left(\mathrm{~s}, 3 \mathrm{H}, \mathrm{CH}_{3}\right) .{ }^{13} \mathrm{C} \mathrm{NMR}\left(101 \mathrm{MHz}, \mathrm{CDCl}_{3}\right) \delta 132.3,124.6,109.5,106.0$, 80.2, 75.6, 70.7, 50.4, 35.3, 29.9, 23.8. IR 3304 (w), 3292 (w), $2983(\mathrm{~m}), 2942(\mathrm{~m}), 2867(\mathrm{w})$, $2824(\mathrm{w}), 2146(\mathrm{w}), 1653(\mathrm{w}), 1532(\mathrm{w}), 1512(\mathrm{w}), 1486(\mathrm{~m}), 1430(\mathrm{w}), 1411(\mathrm{w}), 1372(\mathrm{w})$, 1353 (w), $1311(\mathrm{~m}), 1294$ (m), 1262 (w), 1239 (w), 1203 (w), 1154 (w), 1099 (s), 1062 (s), $896(\mathrm{w}), 885(\mathrm{w}), 859(\mathrm{w}), 844(\mathrm{~m}), 822(\mathrm{w})$. HRMS (ESI) calcd for $\mathrm{C}_{11} \mathrm{H}_{15} \mathrm{NNaO}^{+}[\mathrm{M}+\mathrm{Na}]^{+}$ 200.1046; found 200.1046.

\section{2-(1-Methoxybut-3-yn-1-yl)-1-methyl-1H-indole (6m)}

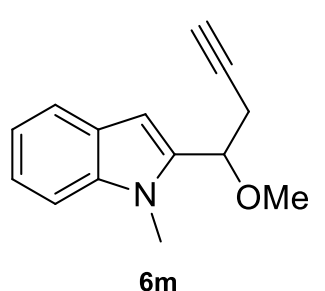

$478 \mathrm{mg}$ of the corresponding aldehyde $(3.00 \mathrm{mmol})$ was used to obtain the crude propargylic alcohol $(500 \mathrm{mg}, 2.51 \mathrm{mmol}$, <84\%), which was used directly in the methylation step. $6 \mathbf{m}$ was obtained as a yellow oil (450 mg, $2.11 \mathrm{mmol}, 70 \%$ over two steps).

Rf (Pentane / EtOAc = $25 / 1): 0.5 .{ }^{1} \mathrm{H}$ NMR $\left(400 \mathrm{MHz}, \mathrm{CDCl}_{3}\right) \delta 7.84$ $(\mathrm{d}, 1 \mathrm{H}, J=7.8 \mathrm{~Hz}$, benzeneH), 7.50-7.43 (m, $2 \mathrm{H}$, benzene $H), 7.35(\mathrm{~m}, 1$ $\mathrm{H}$, benzeneH), $6.73(\mathrm{~s}, 1 \mathrm{H}$, pyrroleH $), 4.89(\mathrm{t}, 1 \mathrm{H}, J=6.9 \mathrm{~Hz}$, $\left.\mathrm{CHOCH}_{3}\right), 3.91\left(\mathrm{~s}, 3 \mathrm{H}, \mathrm{NCH}_{3}\right), 3.47$ (s, $\left.3 \mathrm{H}, \mathrm{OCH}_{3}\right), 3.04$ (ddd, $2 \mathrm{H}, J=16.4,7.3,2.7 \mathrm{~Hz}$, $\left.\mathrm{CH}_{2}\right), 2.29$ (t, $1 \mathrm{H}, J=2.7 \mathrm{~Hz}$, alkyne $\left.\mathrm{CH}\right) .{ }^{13} \mathrm{C} \mathrm{NMR}\left(101 \mathrm{MHz}, \mathrm{CDCl}_{3}\right) \delta 137.9,137.0$, 126.9, 121.5, 120.4, 119.3, 108.8, 101.6, 80.2, 74.7, 70.3, 55.2, 29.9, 24.4. IR 3667 (w), 3479 (w), 2992 (s), 2961 (m), 2906 (m), 1402 (w), 1248 (w), 1236 (w), 1072 (s), 1034 (m), 901 (w), 866 (w). HRMS (ESI) calcd for $\mathrm{C}_{14} \mathrm{H}_{15} \mathrm{NO}[\mathrm{M}]^{+} 214.1226$; found 214.1228.

\section{5-(1-Methoxybut-3-yn-1-yl)-4-methyl-4H-thieno[3,2-b]pyrrole (6n)}

$250 \mathrm{mg}$ of the corresponding aldehyde $(1.51 \mathrm{mmol})$ was used to obtain the

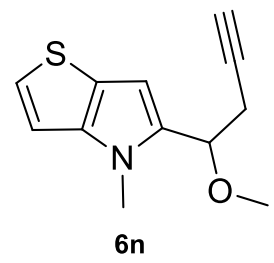
crude propargylic alcohol $(250 \mathrm{mg}, 1.22 \mathrm{mmol},<81 \%) .123 \mathrm{mg}$ of crude propargylic alcohol $(0.599 \mathrm{mmol})$ was used for methylation. 6n was obtained as a yellow oil (93 mg, $0.42 \mathrm{mmol}, 57 \%$ over two steps).

$\operatorname{Rf}($ Pentane / EtOAc = $15 / 1): 0.5 .{ }^{1} \mathrm{H}$ NMR $\left(400 \mathrm{MHz}, \mathrm{CDCl}_{3}\right) \delta 7.10(\mathrm{~d}$, $1 \mathrm{H}, J=5.3 \mathrm{~Hz}$, thiopheneH), $6.94(\mathrm{~d}, 1 \mathrm{H}, J=5.3$, thiopheneH), 6.44 (s, 1 $\mathrm{H}$, pyrroleH), $4.64\left(\mathrm{t}, 1 \mathrm{H}, \mathrm{J}=7.1 \mathrm{~Hz}, \mathrm{CHOCH}_{3}\right), 3.81\left(\mathrm{~s}, 3 \mathrm{H}, \mathrm{NCH}_{3}\right), 3.32$ (s, $\left.3 \mathrm{H}, \mathrm{OCH}_{3}\right), 2.84\left(\mathrm{ddd}, 2 \mathrm{H}, J=15.2,7.3,2.7 \mathrm{~Hz}, \mathrm{CH}_{2}\right), 2.06(\mathrm{t}, 1 \mathrm{H}, J=2.7 \mathrm{~Hz}$, alkyne $\mathrm{CH}) .{ }^{13} \mathrm{C}$ NMR $\left(101 \mathrm{MHz}, \mathrm{CDCl}_{3}\right) \delta 142.0,135.3,123.3,121.4,109.8,100.9,80.5,75.1,70.2$, 55.4, 32.6, 24.7. IR 3285 (w), 2934 (w), 2821 (w), 1530 (w), 1399 (w), $1354(w), 1319(w)$, $1187(\mathrm{w}), 1135$ (w), $1095(\mathrm{~m}), 1085(\mathrm{~m}), 1006(\mathrm{w}), 840(\mathrm{w}), 769$ (w), $714(\mathrm{~s}), 687(\mathrm{~m}), 677$ (s), $663(\mathrm{~s}), 655(\mathrm{~s}), 613(\mathrm{w})$. HRMS (ESI) calcd for $\mathrm{C}_{12} \mathrm{H}_{14} \mathrm{NOS}^{+}[\mathrm{M}+\mathrm{H}]^{+} 220.0791$; found 220.0786 .

\section{3-(1-Methoxybut-3-yn-1-yl)-1-methyl-1H-pyrrole (9a)}

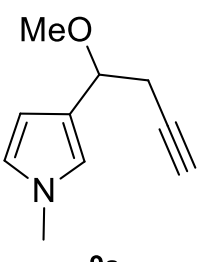

$610 \mathrm{mg}$ of 29 (5.59 mmol) was used to obtain the crude propargylic alcohol (600 mg, $4.02 \mathrm{mmol},<72 \%$ ), which was used directly in the methylation step. 9a was obtained as yellow oil (480 mg, $2.94 \mathrm{mmol}, 53 \%$ over two steps).

Rf (Pentane / EtOAc = $15 / 1): 0.6 .{ }^{1} \mathrm{H}$ NMR $\left(400 \mathrm{MHz}, \mathrm{CDCl}_{3}\right) \delta 6.60(\mathrm{~s}, 1 \mathrm{H}$, pyrroleH ), $6.54(\mathrm{t}, 1 \mathrm{H}, J=2.5 \mathrm{~Hz}$, pyrroleH), $6.11(\mathrm{~m}, 1 \mathrm{H}$, pyrroleH $), 4.28(\mathrm{t}$,

$\left.1 \mathrm{H}, J=6.5 \mathrm{~Hz}, \mathrm{CHOCH}_{3}\right), 3.59\left(\mathrm{~s}, 3 \mathrm{H}, \mathrm{NCH}_{3}\right), 3.27\left(\mathrm{~s}, 3 \mathrm{H}, \mathrm{OCH}_{3}\right), 2.64$ (ddd, $\left.2 \mathrm{H}, J=16.4,6.2,2.7 \mathrm{~Hz}, \mathrm{CH}_{2}\right), 1.97(\mathrm{t}, 1 \mathrm{H}, J=2.6 \mathrm{~Hz}$, alkyne $\mathrm{CH}) .{ }^{13} \mathrm{C} \mathrm{NMR}(101 \mathrm{MHz}$, $\left.\mathrm{CDCl}_{3}\right) \delta 122.8,121.4,119.6,106.3,81.4,75.5,69.0,55.5,35.5,26.6$. IR $3303(\mathrm{w}), 2939(\mathrm{~m})$, 
$2868(\mathrm{w}), 2820(\mathrm{w}), 1558(\mathrm{w}), 1509(\mathrm{w}), 1466(\mathrm{w}), 1424(\mathrm{w}), 1319(\mathrm{w}), 1270(\mathrm{w}), 1165(\mathrm{w})$, 1164 (w), 1099 (s), 1005 (w), 822 (w), 787 (w), 740 (m), 706 (w). HRMS (ESI) calcd for $\mathrm{C}_{10} \mathrm{H}_{13} \mathrm{NNaO}^{+}[\mathrm{M}+\mathrm{Na}]^{+}$186.0889; found 186.0895.

\section{3-(1-Methoxypent-3-yn-1-yl)-1-methyl-1H-pyrrole (9b)}
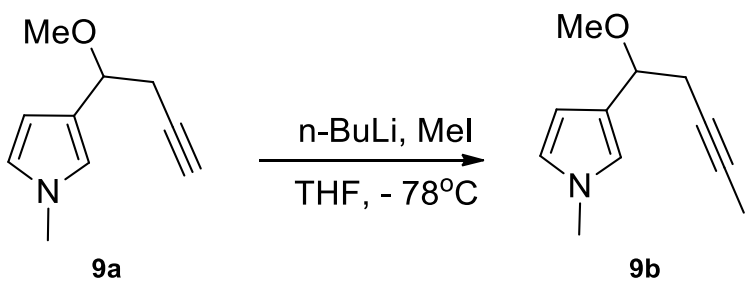

BuLi (0.386 mL, $0.965 \mathrm{mmol}, 1.05$ equiv) was added to a solution of 9a (150 mg, 0.919 mmol, 1.0 equiv) in dry THF $(3 \mathrm{~mL})$ at $-78{ }^{\circ} \mathrm{C}$. The solution was kept during 30 minutes at $78{ }^{\circ} \mathrm{C}$ and 15 minutes at room temperature. Then the solution was re-cooled down to $-78{ }^{\circ} \mathrm{C}$, MeI (272 mg, $1.92 \mathrm{mmol}, 1.0$ equiv) was added dropwise. The reaction was quenched with a sat $\mathrm{NH}_{4} \mathrm{Cl}$ solution $(30 \mathrm{~mL}) 2$ hours later. The aqueous and organic layers were separated and the aqueous layer was extracted with ether $(3 \times 10 \mathrm{~mL})$. After drying over $\mathrm{MgSO}_{4}$ and concentrating under vacuum, the crude product was purified by column chromatography $\left(\right.$ Pentane/EtOAc $\left.=25: 1,1 \% \mathrm{Et}_{3} \mathrm{~N}\right)$ to afford $9 \mathrm{~b}$ as brown oil $(89 \mathrm{mg}, 0.50 \mathrm{mmol}, 55 \%)$

Rf (Pentane/EtOAc= 25:1): 0.3. ${ }^{1} \mathrm{H}$ NMR $\left(400 \mathrm{MHz} \mathrm{CDCl}_{3}\right) \delta 6.58(\mathrm{t}, 1 \mathrm{H}, J=1.9 \mathrm{~Hz}$, pyrroleH), $6.54(\mathrm{t}, 1 \mathrm{H}, J=2.5 \mathrm{~Hz}$, pyrrole $\mathrm{H}), 6.11(\mathrm{~m}, 1 \mathrm{H}$, pyrroleH $), 4.22(\mathrm{t}, 1 \mathrm{H}, J=6.3$ $\left.\mathrm{Hz}, \mathrm{CHOCH}_{3}\right), 3.62\left(\mathrm{~s}, 3 \mathrm{H}, \mathrm{NCH}_{3}\right), 3.28\left(\mathrm{~s}, 3 \mathrm{H}, \mathrm{OCH}_{3}\right), 2.67-2.50\left(\mathrm{~m}, 2 \mathrm{H}, \mathrm{CH}_{2}\right), 1.78$ (t, 3 $\mathrm{H}, J=2.5 \mathrm{~Hz}$, alkyne $\left.\mathrm{CH}_{3}\right) .{ }^{13} \mathrm{C} \mathrm{NMR}\left(101 \mathrm{MHz}, \mathrm{CDCl}_{3}\right) \delta 123.6,121.6,119.8,106.6,76.4$, 76.4, 76.3, 56.0, 35.9, 27.3, 3.5. IR 3667 (w), 3522 (w), 3512 (w), 3489 (w), 3479 (w), 2992 (s), 2961 (m), 2960 (m), 2906 (m), 1402 (w), 1248 (w), 1072 (s), 1034 (m), 900 (w), 865 (w), 746 (w). HRMS (ESI) calcd for $\mathrm{C}_{11} \mathrm{H}_{15} \mathrm{NNaO}^{+}[\mathrm{M}+\mathrm{Na}]^{+}$200.1046; found 200.1047.

\section{3-(1-Methoxy-4-phenylbut-3-yn-1-yl)-1-methyl-1H-pyrrole (9c)}

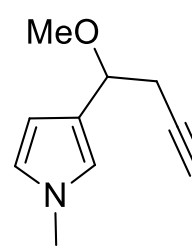

$9 a$<smiles>Ic1ccccc1</smiles>

31

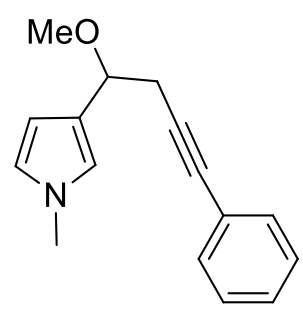

9c

Following the conditions described for $6 \mathrm{~g}$, 9a $(289 \mathrm{mg}, 1.77 \mathrm{mmol})$ was used. The crude product was purified by flash column chromatography (Pentane/EtOAc $\left.=50: 1,1 \% \mathrm{Et}_{3} \mathrm{~N}\right)$ to afford 9c as yellow oil (334 $\mathrm{mg}, 1.40 \mathrm{mmol}, 79 \%)$.

Rf $($ Pentane / EtOAc = $50 / 1): 0.4 .{ }^{1} \mathrm{H}$ NMR $\left(400 \mathrm{MHz}, \mathrm{CDCl}_{3}\right) \delta$ 7.50-7.47 (m, $2 \mathrm{H}$, benzeneH), 7.34-7.31 (m, $3 \mathrm{H}$, benzene $H), 6.69(\mathrm{t}, 1 \mathrm{H}, J=1.8 \mathrm{~Hz}$, pyrroleH), $6.62(\mathrm{t}, 1 \mathrm{H}, J$ $=2.4 \mathrm{~Hz}$, pyrroleH $), 6.26(\mathrm{~m}, 1 \mathrm{H}$, pyrroleH $), 4.45\left(\mathrm{t}, 1 \mathrm{H}, J=6.4 \mathrm{~Hz}, \mathrm{CHOCH}_{3}\right), 3.63(\mathrm{~s}, 3 \mathrm{H}$, $\left.\mathrm{NCH}_{3}\right), 3.41\left(\mathrm{~s}, 3 \mathrm{H}, \mathrm{OCH}_{3}\right), 2.96\left(\mathrm{dd}, 2 \mathrm{H}, \mathrm{J}=16.2,6.7 \mathrm{~Hz}, \mathrm{CH}_{2}\right) .{ }^{13} \mathrm{C}$ NMR $(101 \mathrm{MHz}$, $\left.\mathrm{CDCl}_{3}\right) \delta 131.3,127.8,127.2,123.8,123.4,121.6,119.7,106.6,87.5,81.4,76.2,56.0,35.7$, 28.1. IR 2936 (w), 2935 (w), 2243 (w), 1508 (w), 1495 (w), 1163 (w), 1096 (m), 909 (s), 785 (w), 729 (s). HRMS (ESI) calcd for $\mathrm{C}_{16} \mathrm{H}_{17} \mathrm{NO}^{+}[\mathrm{M}+]$ 262.1202; found 262.1207. 


\section{3-(1-Methoxy-4-(naphthalen-1-yl)but-3-yn-1-yl)-1-methyl-1H-pyrrole (9d)}<smiles>C#CCC(OC)c1ccn(C)c1</smiles>

9a<smiles>Ic1cccc2ccccc12</smiles>

33

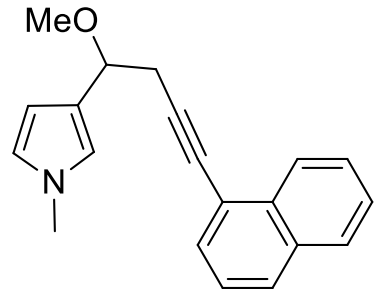

9d

Following the conditions described for $\mathbf{6 g}$, 9a $(163 \mathrm{mg}, 1.00 \mathrm{mmol})$ was used. The crude product was purified by flash column chromatography (Pentane/EtOAc $\left.=50: 1,1 \% \mathrm{Et}_{3} \mathrm{~N}\right)$ to afford 9d as yellow oil (210 $\mathrm{mg}, 0.730 \mathrm{mmol}, 73 \%)$.

Rf (Pentane / EtOAc = 50 / 1): 0.4. ${ }^{1} \mathrm{H}$ NMR (400 MHz, $\left.\mathrm{CDCl}_{3}\right) \delta$ 8.11-8.08 (m, $1 \mathrm{H}$, benzeneH), 7.71-7.69 (m, $1 \mathrm{H}$, benzeneH), $7.65(\mathrm{~d}, 1 \mathrm{H}, J=8.3 \mathrm{~Hz}$, benzeneH $), 7.51(\mathrm{~d}, 1 \mathrm{H}, J$ = 7.1 Hz, benzeneH $), 7.41-7.37(\mathrm{~m}, 2 \mathrm{H}$, benzeneH $), 7.27(\mathrm{~m}, 1 \mathrm{H}$, benzeneH $), 6.58(\mathrm{~s}, 1 \mathrm{H}$, pyrroleH), $6.50(\mathrm{t}, 1 \mathrm{H}, J=2.3 \mathrm{~Hz}$, pyrrole $\mathrm{H}), 6.14(\mathrm{~m}, 1 \mathrm{H}$, pyrroleH $), 4.36(\mathrm{t}, 1 \mathrm{H}, J=6.6$ $\left.\mathrm{Hz}, \mathrm{CHOCH}_{3}\right), 3.52\left(\mathrm{~s}, 3 \mathrm{H}, \mathrm{NCH}_{3}\right), 3.28\left(\mathrm{~s}, 3 \mathrm{H}, \mathrm{OCH}_{3}\right), 2.94(\mathrm{dd}, 2 \mathrm{H}, J=16.6,6.8 \mathrm{~Hz}$, $\left.\mathrm{CH}_{2}\right) .{ }^{13} \mathrm{C}$ NMR $\left(101 \mathrm{MHz}, \mathrm{CDCl}_{3}\right) \delta 133.6,133.1,129.8,128.0,127.9,126.5,126.2,126.1$, 125.1, 123.6, 121.9, 121.6, 120.2, 106.9, 92.6, 79.7, 76.5, 56.3, 36.1, 28.4. IR 3052 (w), 2975 (w), $1735(\mathrm{w}), 1583(\mathrm{w}), 1555(\mathrm{w}), 1507(\mathrm{w}), 1218(\mathrm{w}), 1163(\mathrm{~m}), 1094(\mathrm{~s}), 1006(\mathrm{w}), 962$ (w), $914(\mathrm{w}), 799$ (s), 776 (s), 703 (w). HRMS (ESI) calcd for $\mathrm{C}_{20} \mathrm{H}_{20} \mathrm{NO}^{+}[\mathrm{M}+]$ 312.1359; found 312.1356 .

\section{3-(2-Methoxypent-4-yn-2-yl)-1-methyl-1H-pyrrole (9e)}

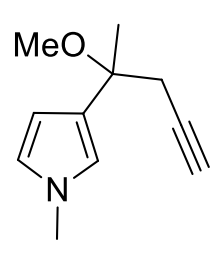

$9 e$

$616 \mathrm{mg}$ of the corresponding ketone $(5.00 \mathrm{mmol})$ was used to obtain the crude propargylic alcohol $(555 \mathrm{mg}, 3.40 \mathrm{mmol},<68 \%) .326 \mathrm{mg}$ of crude propargylic alcohol $(2.00 \mathrm{mmol})$ was used for methylation. 9e was obtained as a transparent oil (297 mg, $1.68 \mathrm{mmol}, 57 \%$ over two steps).

Rf (Pentane / EtOAc = $15 / 1): 0.6 .{ }^{1} \mathrm{H}$ NMR $\left(400 \mathrm{MHz}, \mathrm{CDCl}_{3}\right) \delta 6.55(\mathrm{~d}, 2 \mathrm{H}$, $J=2.3 \mathrm{~Hz}$, pyrroleH), $6.10\left(\mathrm{t}, 1 \mathrm{H}, J=2.3 \mathrm{~Hz}\right.$, pyrroleH), $3.62\left(\mathrm{~s}, 3 \mathrm{H}, \mathrm{NCH}_{3}\right)$, 3.09 (s, $\left.3 \mathrm{H}, \mathrm{OCH}_{3}\right), 2.68\left(\mathrm{dd}, 2 \mathrm{H}, J=16.0 \mathrm{~Hz}, 2.7 \mathrm{~Hz}, \mathrm{CH}_{2}\right), 2.00(\mathrm{t}, 1 \mathrm{H}, J=2.7 \mathrm{~Hz}$, alkyne $\mathrm{CH}), 1.60\left(\mathrm{~s}, 3 \mathrm{H}, \mathrm{CH}_{3}\right) .{ }^{13} \mathrm{C} \mathrm{NMR}\left(101 \mathrm{MHz}, \mathrm{CDCl}_{3}\right) \delta 126.3,121.6,119.5,106.8,81.3,74.6$, 70.0, 50.1, 35.9, 31.8, 23.5. IR $3686(\mathrm{w}), 3674(\mathrm{w}), 3296(\mathrm{w}), 3285(\mathrm{w}), 2987(\mathrm{~s}), 2941(\mathrm{~m})$, 2940 (m), 2910 (m), 2902 (s), 2822 (w), 1556 (w), 1509 (w), 1473 (w), 1456 (w), 1420 (w), 1407 (w), 1394 (w), 1378 (w), 1258 (w), 1251 (w), 1243 (w), 1233 (w), $1210(\mathrm{w}), 1191$ (w), 1098 (s), 1092 (s), 1067 (s), 1059 (s), 1017 (w), 787 (w), 744 (m), 699 (w), 682 (w), 660 (s), $652(\mathrm{~m}), 644(\mathrm{~s}), 633(\mathrm{~m}), 622(\mathrm{w}), 606(\mathrm{w})$. HRMS (ESI) calcd for $\mathrm{C}_{11} \mathrm{H}_{15} \mathrm{NNaO}^{+}[\mathrm{M}+\mathrm{Na}]^{+}$ 200.1046; found 200.1039.

\section{3-(1-Methoxy-1-phenylbut-3-yn-1-yl)-1-methyl-1H-pyrrole (9f)}

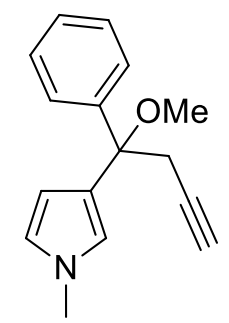

$9 f$

$460 \mathrm{mg}$ of the corresponding ketone $(2.48 \mathrm{mmol})$ was used to obtain the crude propargylic alcohol (500 mg, $2.20 \mathrm{mmol},<89 \%)$. $250 \mathrm{mg}$ of crude propargylic alcohol $(1.11 \mathrm{mmol})$ was used for methylation. 9f was obtained as yellow oil (106 mg, $0.443 \mathrm{mmol}, 36 \%$ over two steps).

$\operatorname{Rf}($ Pentane / EtOAc = $25 / 1): 0.8 .{ }^{1} \mathrm{H}$ NMR $\left(400 \mathrm{MHz}, \mathrm{CDCl}_{3}\right) \delta 7.51(\mathrm{~m}, 2$ $\mathrm{H}$, benzene $\mathrm{H}), 7.33(\mathrm{~m}, 2 \mathrm{H}$, benzene $H), 7.26(\mathrm{~m}, 1 \mathrm{H}$, benzene $H), 6.56(\mathrm{~m}, 2$ $\mathrm{H}$, pyrroleH), $6.04(\mathrm{dd}, 1 \mathrm{H}, J=2.6,2.0 \mathrm{~Hz}$, pyrrole $\mathrm{H}), 3.63\left(\mathrm{~s}, 3 \mathrm{H}, \mathrm{NCH}_{3}\right)$, $3.18\left(\mathrm{~s}, 3 \mathrm{H}, \mathrm{OCH}_{3}\right), 3.08\left(\mathrm{dd}, 2 \mathrm{H}, J=16.0 \mathrm{~Hz}, 2.7 \mathrm{~Hz}, \mathrm{CH}_{2}\right), 1.93(\mathrm{t}, 1 \mathrm{H}, J=$ $2.7 \mathrm{~Hz}$, alkyne $\mathrm{CH}) .{ }^{13} \mathrm{C} \mathrm{NMR}\left(101 \mathrm{MHz}, \mathrm{CDCl}_{3}\right) \delta 144.0,127.5,127.1,126.8$, 
125.5, 121.6, 121.3, 108.6, 80.8, 79.5, 70.8, 51.0, 36.2, 30.8. IR $3288(\mathrm{w}), 3287(\mathrm{w}), 3057(\mathrm{w})$, 2938 (w), 2918 (w), 2823 (w), $1544(\mathrm{w}), 1509(\mathrm{w}), 1492(\mathrm{w}), 1472(\mathrm{w}), 1447(\mathrm{w}), 1422(\mathrm{w})$, 1263 (w), 1200 (w), 1166 (m), 1089 (s), 1033 (w), 1015 (w), 864 (w), 785 (m), 765 (m), 707 (s). HRMS (ESI) calcd for $\mathrm{C}_{16} \mathrm{H}_{18} \mathrm{NO}^{+}[\mathrm{M}+\mathrm{H}]^{+} 240.1383$; found 240.1378 .

\section{3-(2-Methoxyhex-4-yn-2-yl)-1-methyl-1H-pyrrole (9g)}

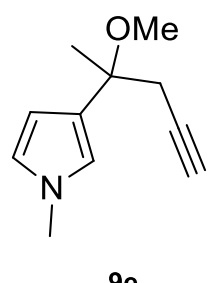

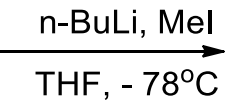

$9 e$

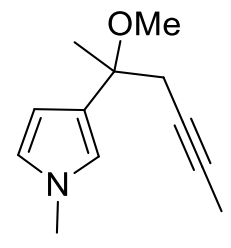

$9 \mathrm{~g}$

Starting from 9e (340 mg, $1.92 \mathrm{mmol}, 1.0$ equiv) and following the conditions described for 9b, the crude product was purified by flash column chromatography (Pentane/EtOAc $=25: 1$, $\left.1 \% \mathrm{Et}_{3} \mathrm{~N}\right)$ to afford $9 \mathrm{~g}$ as yellow oil $(192 \mathrm{mg}, 1.00 \mathrm{mmol}, 52 \%)$

Rf (Pentane/EtOAc= 25:1): 0.5. ${ }^{1} \mathrm{H}$ NMR $\left(400 \mathrm{MHz} \mathrm{CDCl}_{3}\right) \delta 6.54(\mathrm{~m}, 2 \mathrm{H}$, pyrroleH $), 6.10$ (m, $1 \mathrm{H}$, pyrroleH), 3.63 (s, $\left.3 \mathrm{H}, \mathrm{NCH}_{3}\right), 3.09\left(\mathrm{~s}, 3 \mathrm{H}, \mathrm{OCH}_{3}\right), 2.64\left(\mathrm{~m}, 2 \mathrm{H}, \mathrm{CH}_{2}\right), 1.80(\mathrm{t}, 3 \mathrm{H}$, $J=2.6 \mathrm{~Hz}$, alkyne $\left.\mathrm{CH}_{3}\right), 1.56\left(\mathrm{~s}, 3 \mathrm{H}, \mathrm{CH}_{3} \mathrm{COCH}_{3}\right) .{ }^{13} \mathrm{C} \mathrm{NMR}\left(101 \mathrm{MHz}, \mathrm{CDCl}_{3}\right) \delta 127.1$, 121.6, 119.7, 107.1, 77.4, 76.0, 75.1, 50.4, 36.2, 32.1, 23.9, 3.8. IR 2979 (m), 2933 (w), 2910 (w), $2820(\mathrm{w}), 1552(\mathrm{w}), 1509(\mathrm{~m}), 1484(\mathrm{w}), 1423(\mathrm{~m}), 1371(\mathrm{~m}), 1307(\mathrm{w}), 1262(\mathrm{~m}), 1237$ (w), 1208 (m), 1191 (m), 1152 (w), 1093 (s), 1069 (m), 965 (w), 914 (m), 832 (w), 814 (w), 783 (s), 755 (w), 737 (m), 705 (w). HRMS (ESI) calcd for $\mathrm{C}_{12} \mathrm{H}_{17} \mathrm{NNaO}^{+}[\mathrm{M}+\mathrm{Na}]^{+}$214.1202; found 214.1200.

\section{Methylpent-4-yn-2-yl)-1-methyl-1H-pyrrole (9h)}

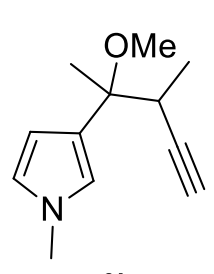

$616 \mathrm{mg}$ of the corresponding ketone $(5.00 \mathrm{mmol})$ and 3-bromobut-1-yne $(864$ $\mathrm{mg}, 6.50 \mathrm{mmol})$ were used to obtain the crude propargylic alcohol $(777 \mathrm{mg}$, $4.76 \mathrm{mmol},<95 \%)$, which was used directly in the methylation step. 9h was obtained as yellow oil $(551 \mathrm{mg}, 2.88 \mathrm{mmol}, 58 \%$ over two steps, $\mathrm{dr}=2: 1$, obtained by integration of the ${ }^{1} \mathrm{H}$ NMR peaks at 6.11 and 6.04).

$9 \mathrm{~h}$

Rf $($ Pentane / EtOAc = $25 / 1): 0.5 .{ }^{1} \mathrm{H}$ NMR $\left(400 \mathrm{MHz}, \mathrm{CDCl}_{3}\right) \delta 6.57(\mathrm{t}, 1 \mathrm{H}$, $J=2.1 \mathrm{~Hz}$, pyrroleH major diastereoisomer), $6.54(\mathrm{t}, 1.5 \mathrm{H}, J=2.1 \mathrm{~Hz}$, pyrroleH major and minor diastereoisomer), 6.48 (t, $0.5 \mathrm{H}, J=2.1 \mathrm{~Hz}$, pyrrole $H$ minor diastereoisomer), 6.11 (dd, $1 \mathrm{H}, J=2.7,1.8 \mathrm{~Hz}$, pyrroleH major diastereoisomer), 6.04 (dd, $0.5 \mathrm{H}, J=2.7,1.8 \mathrm{~Hz}$, pyrroleH minor diastereoisomer), $3.62\left(\mathrm{~s}, 3 \mathrm{H}, \mathrm{NCH}_{3}\right.$ major diastereoisomer), $3.60(\mathrm{~s}, 1 \mathrm{H}$, $\mathrm{NCH}_{3}$ minor diastereoisomer), 3.12 (s, $1 \mathrm{H}, \mathrm{OCH}_{3}$ minor diastereoisomer), 3.08 (s, $3 \mathrm{H}, \mathrm{OCH}_{3}$ major diastereoisomer), 2.89 (m, $1.5 \mathrm{H}, \mathrm{CH}_{3} \mathrm{CH}$ major and minor diastereoisomer), 2.11 (d, $0.5 \mathrm{H}, J=2.5 \mathrm{~Hz}$, alkyne $C H$ minor diastereoisomer), 2.01 (d, $1 \mathrm{H}, J=2.5 \mathrm{~Hz}$, alkyne $C H$ major diastereoisomer), 1.53 (two s, $4.5 \mathrm{H}, \mathrm{CH}_{3} \mathrm{OMe}$ major and minor diastereoisomer), 1.23 $\left(\mathrm{d}, 3 \mathrm{H}, \mathrm{J}=7.0 \mathrm{~Hz}, \mathrm{CH}_{3} \mathrm{CH}\right.$ major diastereoisomer), $1.03\left(\mathrm{~d}, 1.5 \mathrm{H}, J=7.0 \mathrm{~Hz}, \mathrm{CH}_{3} \mathrm{CH}\right.$ minor diastereoisomer). ${ }^{13} \mathrm{C}$ NMR $\left(101 \mathrm{MHz}, \mathrm{CDCl}_{3}\right) \delta 126.0,124.9,121.4,121.1,120.4,120.3$, $107.5,107.4,87.2,86.9,77.9,77.2,69.6,69.5,50.1,50.0,37.5,36.8,36.0,35.9,19.6,18.2$, 16.6, 15.6. IR 3308 (w), 2983 (w), 2982 (w), 2940 (w), 1508 (w), 1374 (w), 1219 (w), 1196 (w), $1089(w), 1074(w), 909$ (s), $780(w), 729$ (s), 706 (w). HRMS (ESI) calcd for $\mathrm{C}_{12} \mathrm{H}_{17} \mathrm{NNaO}^{+}[\mathrm{M}+\mathrm{Na}]^{+}$214.1202; found 214.1204.

\section{3-(1-Methoxybut-3-yn-1-yl)-1-methyl-1H-indole (9i)}




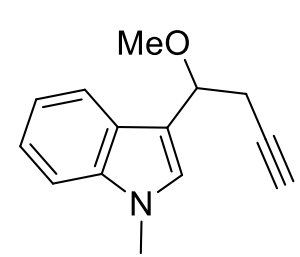

$9 \mathbf{i}$

$970 \mathrm{mg}$ of the corresponding aldehyde $(6.09 \mathrm{mmol})$ was used to obtain the crude propargylic alcohol (1.03 g, $5.14 \mathrm{mmol},<84 \%) .398 \mathrm{mg}$ of crude propargylic alcohol $(2.00 \mathrm{mmol})$ was used for methylation. 9i was obtained as a yellow oil (372 $\mathrm{mg}, 1.74 \mathrm{mmol}, 73 \%$ over two steps).

Rf (Pentane / EtOAc = $15 / 1): 0.2 .{ }^{1} \mathrm{H}$ NMR $\left(400 \mathrm{MHz}, \mathrm{CDCl}_{3}\right) \delta 7.85(\mathrm{~m}$, $1 \mathrm{H}$, benzeneH), 7.40-7.32 (m, $2 \mathrm{H}$, benzeneH), $7.23(\mathrm{~m}, 1 \mathrm{H}$, benzeneH), $7.14(\mathrm{~s}, 1 \mathrm{H}$, pyrroleH $), 4.79\left(\mathrm{t}, 1 \mathrm{H}, J=6.5 \mathrm{~Hz}, \mathrm{CHOCH}_{3}\right), 3.80(\mathrm{~s}, 3 \mathrm{H}$, $\left.\mathrm{NCH}_{3}\right), 3.42\left(\mathrm{~s}, 3 \mathrm{H}, \mathrm{OCH}_{3}\right), 2.95\left(\mathrm{ddd}, 2 \mathrm{H}, \mathrm{J}=16.8,6.3,2.7 \mathrm{~Hz}, \mathrm{CH}_{2}\right), 2.11(\mathrm{t}, 1 \mathrm{H}, \mathrm{J}=2.6$ $\mathrm{Hz}$, alkyne $\mathrm{CH}) .{ }^{13} \mathrm{C} \mathrm{NMR}\left(101 \mathrm{MHz}, \mathrm{CDCl}_{3}\right) \delta 137.0,127.2,126.2,121.6,119.5,119.1$, 113.5, 109.2, 81.6, 75.7, 69.6, 56.2, 32.5, 26.7. IR 3308 (w), 2341 (w), 1473 (w), 1378 (w), $1331(\mathrm{w}), 1243$ (w), $1096(\mathrm{~m}), 1068$ (w), 907 (s), 726 (s), 685 (w), 672 (w), $660(\mathrm{~m}), 634(\mathrm{~m})$. HRMS (ESI) calcd for $\mathrm{C}_{14} \mathrm{H}_{15} \mathrm{NNaO}^{+}[\mathrm{M}+\mathrm{Na}]^{+} 236.1046$; found 236.1048.

\section{5-methoxy-3-(1-methoxybut-3-yn-1-yl)-1-methyl-1H-indole (9j)}

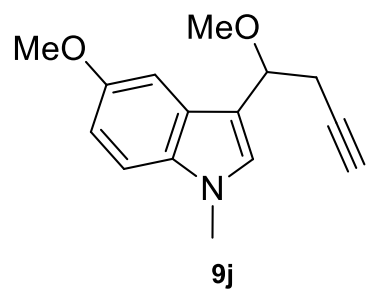

$250 \mathrm{mg}$ of the corresponding aldehyde $(1.32 \mathrm{mmol})$ was used to obtain the crude propargylic alcohol (280 mg, $1.22 \mathrm{mmol},<92 \%)$, which was used directly in the methylation step. $\mathbf{9 j}$ was obtained as a yellow oil (264 mg, $1.09 \mathrm{mmol}, 83 \%$ over two step).

Rf (Pentane / EtOAc = $25 / 1): 0.3 .{ }^{1} \mathrm{H}$ NMR $\left(400 \mathrm{MHz}, \mathrm{CDCl}_{3}\right) \delta$ 7.21-7.18 (m, 2H, benzeneH), 7.04 (s, $1 \mathrm{H}$, pyrroleH), 6.91 (dd, $1 \mathrm{H}, J$ $=8.8,2.5 \mathrm{~Hz}$, benzeneH), $4.65\left(\mathrm{t}, 1 \mathrm{H}, J=6.6 \mathrm{~Hz}, \mathrm{CHOCH}_{3}\right), 3.86\left(\mathrm{~s}, 3 \mathrm{H}, N C H_{3}\right), 3.75(\mathrm{~s}, 3$ $\left.\mathrm{H}, \mathrm{OCH}_{3}\right), 3.33(\mathrm{~s}, 3 \mathrm{H}, \mathrm{CHOCH}), 2.84\left(\mathrm{ddd}, 2 \mathrm{H}, J=16.4,6.1,2.7 \mathrm{~Hz}, \mathrm{CH}_{2}\right), 2.02(\mathrm{t}, 1 \mathrm{H}, J$ $=2.6 \mathrm{~Hz}$, alkyne $\mathrm{CH}) .{ }^{13} \mathrm{C} \mathrm{NMR}\left(101 \mathrm{MHz}, \mathrm{CDCl}_{3}\right) \delta 153.9,132.6,127.9,126.6,113.1$, 112.1, 110.1, 101.5, 81.8, 75.9, 69.6, 56.3, 55.9, 32.9, 26.7. IR 3291 (w), $3284(w), 2989(w)$, $2975(\mathrm{w}), 2935$ (w), 2852 (w), 1623 (w), 1578 (w), 1548 (w), $1491(\mathrm{~s}), 1456(\mathrm{~m}), 1425(\mathrm{~m})$, 1325 (w), 1267 (w), 1224 (s), 1174 (w), 1138 (w), 1123 (w), 1096 (s), 1065 (m), 1035 (w), 912 (w), 875 (w), 819 (w), 797 (m), 734 (m). HRMS (ESI) calcd for $\mathrm{C}_{15} \mathrm{H}_{17} \mathrm{NNaO}_{2}{ }^{+}[\mathrm{M}+\mathrm{Na}]^{+}$ 266.1151 ; found 266.1162 . 


\section{Cyclization-alkynylation domino process.}

\section{Optimization of the reaction:}

A solution of $6 \mathbf{a}(0.1 \mathrm{mmol}, 1.0$ equiv) in solvent $(0.5 \mathrm{~mL})$ was added into a solution of catalyst ( $0.03 \mathrm{mmol}, 0.1$ equiv), base $(0.060 \mathrm{mmol}, 2.0$ equiv) and hypervalent iodine reagent ( $0.600 \mathrm{mmol}, 2$ equiv) in solvent $(0.5 \mathrm{~mL})$. The reaction was stopped after $72 \mathrm{~h}$. The solvent was removed under vaccum, and the crude product was purified by column chromatography (Pentane/DCM=15:1) directly without any further work-up.

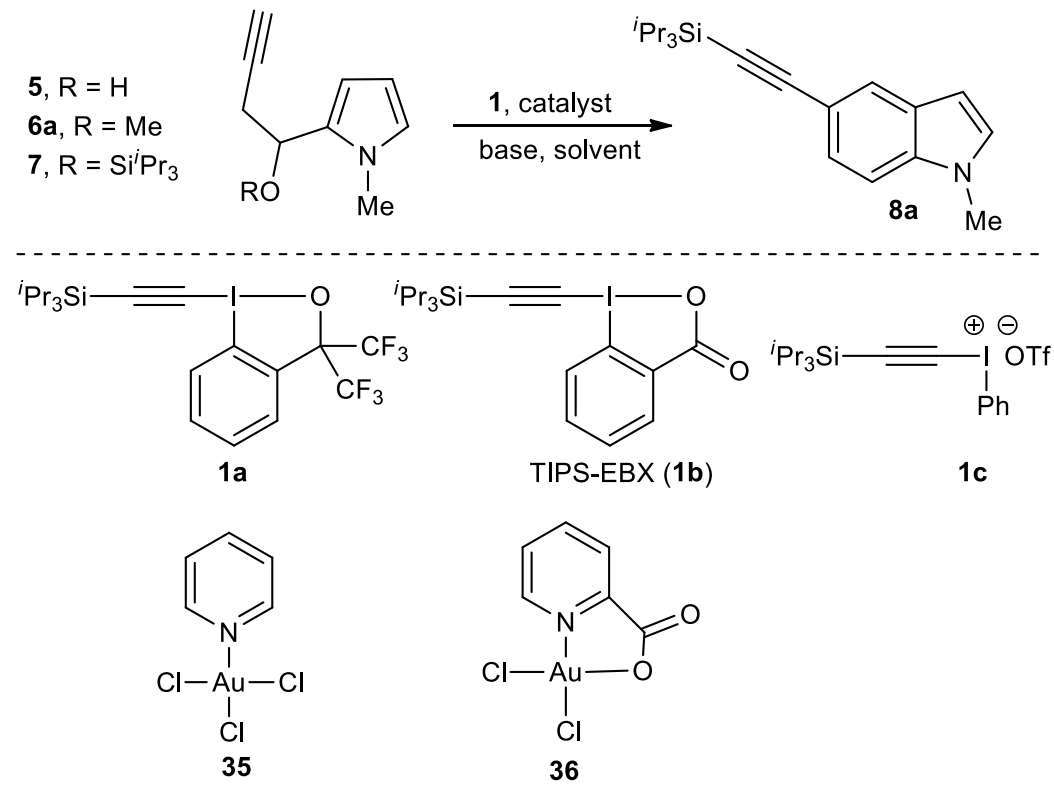

\begin{tabular}{ccccccc}
\hline Entry & Pyrrole & Cat & Reagent & Base & Solvent & Yield \\
\hline 1 & $\mathbf{5}$ & $\mathrm{PPh}_{3} \mathrm{AuCl} / \mathrm{AgOTf}$ & $\mathbf{1 a}$ & $\mathrm{Na}_{2} \mathrm{CO}_{3}$ & $\mathrm{CH}_{3} \mathrm{CN}$ & $<5 \%$ \\
\hline 2 & $\mathbf{5}$ & $\mathrm{AuCl}_{3} / \mathrm{AgOTf}$ & $\mathbf{1 a}$ & $\mathrm{Na}_{2} \mathrm{CO}_{3}$ & $\mathrm{CH}_{3} \mathrm{CN}$ & $<5 \%$ \\
\hline 3 & $\mathbf{5}$ & $\mathrm{AuCl}_{3}$ & $\mathbf{1 a}$ & $\mathrm{Na}_{2} \mathrm{CO}_{3}$ & $\mathrm{CH}_{3} \mathrm{CN}$ & $<5 \%$ \\
\hline 4 & $\mathbf{5}$ & $\mathrm{AuCl}_{3}{ }^{\dagger}$ & $\mathbf{1 a}$ & $\mathrm{Na}_{2} \mathrm{CO}_{3}$ & $\mathrm{CH}_{3} \mathrm{CN}$ & $<5 \%$ \\
\hline 5 & $\mathbf{5}$ & ${\mathrm{Au}(\mathrm{OAc})_{3}{ }^{\dagger}}$ & $\mathbf{1 a}$ & $\mathrm{Na}_{2} \mathrm{CO}_{3}$ & $\mathrm{CH}_{3} \mathrm{CN}$ & $<5 \%$ \\
\hline 6 & $\mathbf{5}$ & $\mathbf{3 5}{ }^{\dagger}$ & $\mathbf{1 a}$ & $\mathrm{Na}_{2} \mathrm{CO}_{3}$ & $\mathrm{CH}_{3} \mathrm{CN}$ & $<5 \%$ \\
\hline 7 & $\mathbf{5}$ & $\mathbf{3 6}{ }^{\dagger}$ & $\mathbf{1 a}$ & $\mathrm{Na}_{2} \mathrm{CO}_{3}$ & $\mathrm{CH}_{3} \mathrm{CN}$ & $<5 \%$ \\
\hline 9 & $\mathbf{5}$ & $\mathrm{PtCl}_{2}$ & $\mathbf{1 a}$ & $\mathrm{Na}_{2} \mathrm{CO}_{3}$ & $\mathrm{CH}_{3} \mathrm{CN}$ & $13 \%$ \\
& $\mathbf{5}$ & $\mathrm{PtCl}_{2} / \mathrm{AgOTf}$ & $\mathbf{1 a}$ & $\mathrm{Na}_{2} \mathrm{CO}_{3}$ & $\mathrm{CH}_{3} \mathrm{CN}$ & $<5 \%$
\end{tabular}




\begin{tabular}{|c|c|c|c|c|c|c|}
\hline 10 & 5 & $\mathrm{PtCl}_{2} /$ bipyridine & $1 a$ & $\mathrm{Na}_{2} \mathrm{CO}_{3}$ & $\mathrm{CH}_{3} \mathrm{CN}$ & $<5 \%$ \\
\hline 11 & 5 & $\mathrm{Pt}\left(\mathrm{NH}_{3}\right)_{2} \mathrm{Cl}_{2}$ & $1 a$ & $\mathrm{Na}_{2} \mathrm{CO}_{3}$ & $\mathrm{CH}_{3} \mathrm{CN}$ & $<5 \%$ \\
\hline 12 & 5 & $\mathrm{PtCl}_{2}(\mathrm{COD})$ & $1 a$ & $\mathrm{Na}_{2} \mathrm{CO}_{3}$ & $\mathrm{CH}_{3} \mathrm{CN}$ & $<5 \%$ \\
\hline 13 & 5 & $\mathrm{PtCl}_{2}$ & $1 a$ & 2.6-lutidine & $\mathrm{CH}_{3} \mathrm{CN}$ & $13 \%$ \\
\hline 14 & 5 & $\mathrm{PtCl}_{2}$ & $1 a$ & $\mathrm{Na}_{3} \mathrm{PO}_{4}$ & $\mathrm{CH}_{3} \mathrm{CN}$ & $15 \%$ \\
\hline 15 & 5 & $\mathrm{PtCl}_{2}$ & $1 a$ & $\mathrm{KHCO}_{3}$ & $\mathrm{CH}_{3} \mathrm{CN}$ & $<5 \%$ \\
\hline 16 & 5 & $\mathrm{PtCl}_{2}$ & $1 a$ & $\mathrm{NaOAc}$ & $\mathrm{CH}_{3} \mathrm{CN}$ & $<5 \%$ \\
\hline 17 & 5 & $\mathrm{PtCl}_{2}$ & $1 a$ & $\mathrm{NaHCO}_{3}$ & $\mathrm{CH}_{3} \mathrm{CN}$ & $40 \%$ \\
\hline 18 & 5 & $\mathrm{PtCl}_{2}$ & $1 a$ & $\mathrm{NaHCO}_{3}$ & Toluene & $30 \%$ \\
\hline 19 & 5 & $\mathrm{PtCl}_{2}$ & $1 a$ & $\mathrm{NaHCO}_{3}$ & THF & $21 \%$ \\
\hline 20 & 5 & $\mathrm{PtCl}_{2}$ & $1 a$ & $\mathrm{NaHCO}_{3}$ & $\mathrm{MeOH}$ & $14 \%$ \\
\hline 21 & 5 & $\mathrm{PtCl}_{2}$ & $1 b$ & $\mathrm{NaHCO}_{3}$ & $\mathrm{CH}_{3} \mathrm{CN}$ & $27 \%$ \\
\hline 22 & 5 & $\mathrm{PtCl}_{2}$ & $1 c$ & $\mathrm{NaHCO}_{3}$ & $\mathrm{CH}_{3} \mathrm{CN}$ & $<5 \%$ \\
\hline 23 & 5 & $\mathrm{PtCl}_{4}$ & $1 a$ & $\mathrm{NaHCO}_{3}$ & $\mathrm{CH}_{3} \mathrm{CN}$ & $<5 \%$ \\
\hline 24 & 5 & $\mathrm{Pt}\left(\mathrm{PPh}_{3}\right)_{4}$ & $1 a$ & $\mathrm{NaHCO}_{3}$ & $\mathrm{CH}_{3} \mathrm{CN}$ & $<5 \%$ \\
\hline 25 & $6 a$ & $\mathrm{PtCl}_{2}$ & $1 a$ & $\mathrm{NaHCO}_{3}$ & $\mathrm{CH}_{3} \mathrm{CN}$ & $91 \% \ddagger$ \\
\hline 26 & 7 & $\mathrm{PtCl}_{2}$ & $1 a$ & $\mathrm{NaHCO}_{3}$ & $\mathrm{CH}_{3} \mathrm{CN}$ & $80 \%$ \\
\hline 27 & 7 & $(\mathrm{IPr}) \mathrm{AuCl} / \mathrm{AgNTf}_{2}$ & $1 a$ & $\mathrm{NaHCO}_{3}$ & $\mathrm{CH}_{3} \mathrm{CN}$ & $<5 \%$ \\
\hline 28 & $6 a$ & $\mathrm{PtBr}_{2}$ & $1 a$ & $\mathrm{NaHCO}_{3}$ & $\mathrm{CH}_{3} \mathrm{CN}$ & $98 \% \ddagger$ \\
\hline 29 & $6 a$ & $\mathrm{Ptl}_{2}$ & $1 a$ & $\mathrm{NaHCO}_{3}$ & $\mathrm{CH}_{3} \mathrm{CN}$ & $<5 \%$ \\
\hline 30 & $6 a$ & $\mathrm{PtBr}_{2}$ & $1 a$ & $\mathrm{NaHCO}_{3}$ & $\begin{array}{c}\mathrm{CH}_{3} \mathrm{CN} / \mathrm{C}_{6} \mathrm{H}_{5} \mathrm{Cl} \\
(\mathrm{v} / \mathrm{v}=1 / 5)\end{array}$ & $45 \%$ \\
\hline 31 & $6 a$ & $\mathrm{PtBr}_{2}$ & $1 a$ & $\mathrm{NaHCO}_{3}$ & $\begin{array}{c}\mathrm{CH}_{3} \mathrm{CN} / \mathrm{THF} \\
(\mathrm{v} / \mathrm{v}=1 / 5)\end{array}$ & $96 \%$ \\
\hline 32 & $6 a$ & $\mathrm{PtBr} 2$ & $1 a$ & $\mathrm{NaHCO}_{3}$ & $\begin{array}{l}\mathrm{CH}_{3} \mathrm{CN} / \mathrm{THF} \\
(\mathrm{v} / \mathrm{v}=1 / 10)\end{array}$ & $93 \%$ \\
\hline
\end{tabular}




\section{General procedure for cyclization-alkynvlation domino process.}

A solution of 6 or $9(0.30 \mathrm{mmol}, 1.0$ equiv) in THF $(1.5 \mathrm{~mL})$ was added into a solution of $\mathrm{PtBr}_{2}$ (11 mg, $0.030 \mathrm{mmol}, 0.1$ equiv), $\mathrm{NaHCO}_{3}(50 \mathrm{mg}, 0.060 \mathrm{mmol}, 2.0$ equiv) and 1[(triisopropylsilyl)ethynyl]-3,3-bis(trifluoromethyl)-3(1H)-1,2-benziodoxole (1a) (330 mg, $0.600 \mathrm{mmol}, 2$ equiv) in THF $(1.0 \mathrm{~mL})$ and $\mathrm{CH}_{3} \mathrm{CN}(0.5 \mathrm{~mL})$. The reaction was stopped after 72h-120h. The solution was removed under vaccum, and the crude product was purified by column chromatography (Pentane/DCM) directly without any further work-up.

\section{1-Methyl-5-((triisopropylsilyl)ethynyl)-1H-indole (8a)}<smiles>C#CCC(OC)c1cccn1C</smiles>

$6 a$

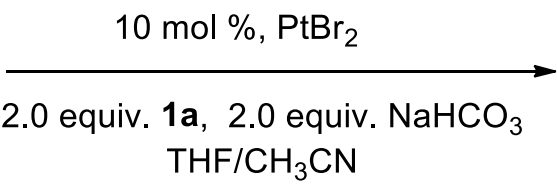

$\mathrm{THF} / \mathrm{CH}_{3} \mathrm{CN}$

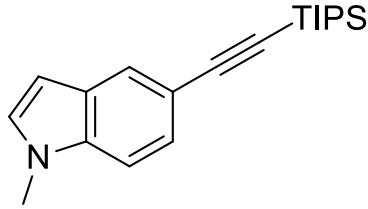

$8 \mathbf{a}$

Starting from 6a (49 $\mathrm{mg}, 0.30 \mathrm{mmol}$ ), the crude product was purified by column chromatography (Pentane/DCM=15:1) to afford 8a as yellow oil (79 $\mathrm{mg}, 0.25 \mathrm{mmol}, 84 \%)$

Rf (Pentane / DCM= $15 / 1): 0.5 .{ }^{1} \mathrm{H}$ NMR (400 MHz, $\left.\mathrm{CDCl}_{3}\right) \delta 7.79(\mathrm{~d}, 1 \mathrm{H}, J=0.7 \mathrm{~Hz}$, benzeneH), $7.34(\mathrm{dd}, 1 \mathrm{H}, J=8.5,1.4 \mathrm{~Hz}$, benzeneH), $7.23(\mathrm{~d}, 1 \mathrm{H}, J=8.5 \mathrm{~Hz}$, benzeneH), $7.05(\mathrm{~d}, 1 \mathrm{H}, J=3.1 \mathrm{~Hz}$, pyrroleH), $6.45(\mathrm{dd}, 1 \mathrm{H}, J=3.1,0.7 \mathrm{~Hz}$, pyrroleH), $3.78(\mathrm{~s}, 3 \mathrm{H}$, $\left.\mathrm{CH}_{3}\right), 1.23(\mathrm{~m}, 21 \mathrm{H}, \mathrm{TIPS}) .{ }^{13} \mathrm{C} \mathrm{NMR}\left(101 \mathrm{MHz}, \mathrm{CDCl}_{3}\right) \delta 136.3,129.7,128.1,125.5,125.2$, 114.1, 109.1, 109.0, 101.2, 87.0, 32.8, 18.7, 11.4. IR 2941 (m), $2863(\mathrm{~m}), 2145(\mathrm{~m}), 1616(\mathrm{w})$, $1514(\mathrm{w}), 1487(\mathrm{~m}), 1464(\mathrm{~m}), 1423(\mathrm{w}), 1381(\mathrm{w}), 1333(\mathrm{w}), 1283(\mathrm{w}), 1147(\mathrm{w}), 1078(\mathrm{w})$, 1014 (w), 996 (w), 945 (w), 919 (w), 884 (s), 820 (s), 802 (m), 717 (s), 710 (s). HRMS (ESI) calcd for $\mathrm{C}_{20} \mathrm{H}_{30} \mathrm{NSi}^{+}[\mathrm{M}+\mathrm{H}]^{+}$312.2142; found 312.2145.

Confirmation of the structure of 1-methyl-5-((triisopropylsilyl)ethynyl)-1H-indole (8a) by Sonogashira coupling

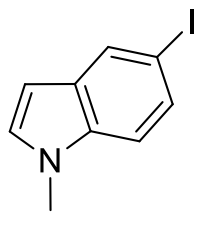

37

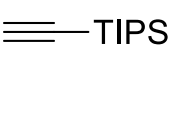

38<smiles>Cn1ccc2cc(C#C[In]S)ccc21</smiles>

$8 \mathbf{a}$

37 (219 mg, $1.20 \mathrm{mmol}, 1.2$ equiv) was added into a solution of $\mathbf{3 6}$ (242 $\mathrm{mg}, 1.00 \mathrm{mmol}, 1.1$ equiv), $\mathrm{Pd}\left(\mathrm{PPh}_{3}\right) \mathrm{Cl}_{2}$ (35 mg, $0.050 \mathrm{mmol}, 0.05$ equiv) and $\mathrm{CuI}$ (38 mg, $0.20 \mathrm{mmol}, 0.2$ equiv) in degazed dioxane $(2 \mathrm{~mL})$. The mixture was kept overnight at $80{ }^{\circ} \mathrm{C}$. The reaction was filtered through a plug of celite and the crude was purified by column chromatography (Pentane/DCM=15:1) to afford $\mathbf{8 a}$ as yellow oil (290 $\mathrm{mg}, 0.930 \mathrm{mmol}, 90 \%)$.

Rf (Pentane / DCM= $15 / 1): 0.5 .{ }^{1} \mathrm{H}$ NMR (400 MHz, $\left.\mathrm{CDCl}_{3}\right) \delta 7.86(\mathrm{~d}, 1 \mathrm{H}, J=0.7 \mathrm{~Hz}$, benzeneH), $7.39(\mathrm{dd}, 1 \mathrm{H}, J=8.5,1.4 \mathrm{~Hz}$, benzeneH), $7.24(\mathrm{~d}, 1 \mathrm{H}, J=8.5 \mathrm{~Hz}$, benzeneH), $7.06(\mathrm{~d}, 1 \mathrm{H}, J=3.1 \mathrm{~Hz}$, pyrroleH), 6.49 (dd, $1 \mathrm{H}, J=3.1,0.7 \mathrm{~Hz}$, pyrroleH), 3.77 (s, $3 \mathrm{H}$, 
$\left.\mathrm{CH}_{3}\right), 1.23(\mathrm{~m}, 21 \mathrm{H}, \mathrm{TIPS}) .{ }^{13} \mathrm{C} \mathrm{NMR}\left(101 \mathrm{MHz}, \mathrm{CDCl}_{3}\right) \delta 136.3,129.7,128.1,125.5,125.2$, 114.1, 109.1, 109.0, 101.2, 87.0, 32.8, 18.7, 11.4. IR 2941 (m), $2863(\mathrm{~m}), 2145$ (m), $1616(\mathrm{w})$, $1514(\mathrm{w}), 1487(\mathrm{~m}), 1464(\mathrm{~m}), 1423(\mathrm{w}), 1381(\mathrm{w}), 1333(\mathrm{w}), 1283(\mathrm{w}), 1147(\mathrm{w}), 1078(\mathrm{w})$, $1014(\mathrm{w}), 996$ (w), 945 (w), $919(\mathrm{w}), 884$ (s), 820 (s), 802 (m), 717 (s), 710 (s). HRMS (ESI) calcd for $\mathrm{C}_{20} \mathrm{H}_{30} \mathrm{NSi}^{+}[\mathrm{M}+\mathrm{H}]^{+}$312.2142; found 312.2150.

\section{1-(p-Tolyl)-5-((triisopropylsilyl)ethynyl)-1H-indole (8b)}<smiles>C#CCC(OC)c1cccn1-c1ccc(C)cc1</smiles>

$6 b$

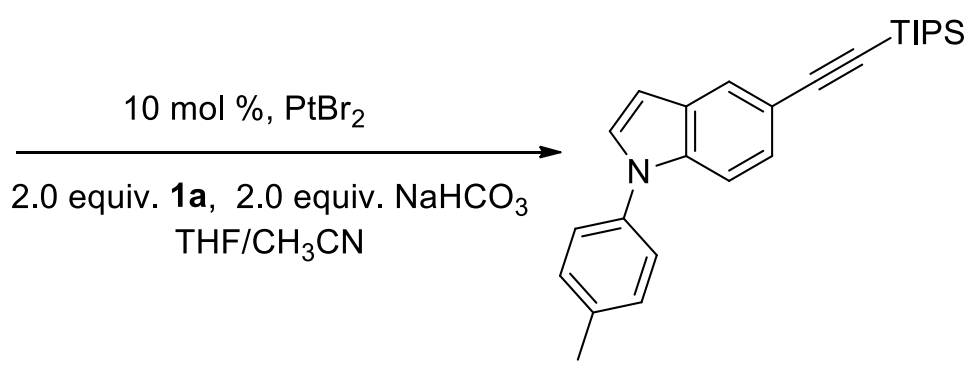

8b

Starting from $\mathbf{6 b}(72 \mathrm{mg}, 0.30 \mathrm{mmol})$, the crude product was purified by column chromatography (Pentane/DCM=15:1) to afford $\mathbf{8 b}$ was obtained as yellow oil (94 mg, 0.24 mmol, $81 \%$ ).

Rf (Pentane / DCM= $15 / 1): 0.5 .{ }^{1} \mathrm{H}$ NMR (400 MHz, $\left.\mathrm{CDCl}_{3}\right) \delta 7.85(\mathrm{~d}, 1 \mathrm{H}, J=0.8 \mathrm{~Hz}$, benzeneH), $7.42(\mathrm{~m}, 1 \mathrm{H}$, benzeneH $), 7.35(\mathrm{~m}, 4 \mathrm{H}$, benzeneH $), 7.31(\mathrm{~d}, 2 \mathrm{H}, J=3.0 \mathrm{~Hz}$, benzene + pyrroleH), $6.63(\mathrm{~d}, 1 \mathrm{H}, J=3.0 \mathrm{~Hz}$, pyrroleH $), 2.44\left(\mathrm{~s}, 3 \mathrm{H}, \mathrm{CH}_{3}\right), 1.15(\mathrm{~m}, 21 \mathrm{H}$, TIPS). ${ }^{13} \mathrm{C}$ NMR $\left(101 \mathrm{MHz}, \mathrm{CDCl}_{3}\right) \delta 136.9,136.6,135.6,130.2,129.0,128.9,126.2,125.4$, 124.3, 115.1, 110.3, 108.7, 103.3, 87.5, 21.1, 18.7, 11.4. IR 2944 (w), 2865 (w), 2149 (w), $1611(\mathrm{w}), 1520(\mathrm{~m}), 1471(\mathrm{~m}), 1336(\mathrm{w}), 1222(\mathrm{w}), 884(\mathrm{w}), 806(\mathrm{w}), 762(\mathrm{w}), 743(\mathrm{w}), 721$ $(\mathrm{w}), 680(\mathrm{~m}), 660(\mathrm{w}), 647(\mathrm{w}), 631(\mathrm{w}), 618(\mathrm{w})$. HRMS (ESI) calcd for $\mathrm{C}_{26} \mathrm{H}_{34} \mathrm{NSi}^{+}[\mathrm{M}+\mathrm{H}]^{+}$ 388.2455; found 388.2460.

\section{1-Benzyl-5-((triisopropylsilyl)ethynyl)-1H-indole (8c)}

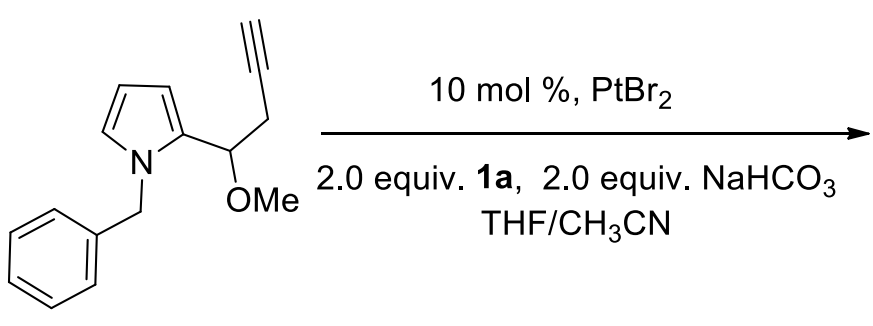

6c

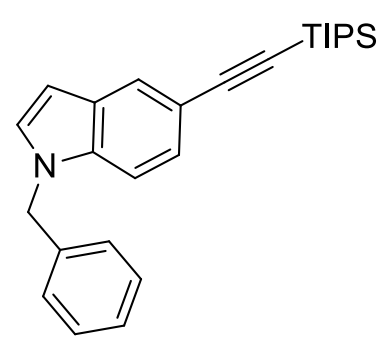

8c

Starting from 6c $(76 \mathrm{mg}, 0.30 \mathrm{mmol})$, the crude product was purified by column chromatography (Pentane/DCM= 30:1) to afford 8c as yellow oil (96 mg, $0.25 \mathrm{mmol}, 83 \%)$.

Rf (Pentane / DCM= 30 / 1): 0.4. ${ }^{1} \mathrm{H}$ NMR $\left(400 \mathrm{MHz}, \mathrm{CDCl}_{3}\right) \delta 7.84(\mathrm{dd}, 1 \mathrm{H}, J=1.4,0.6 \mathrm{~Hz}$, benzeneH), $7.30(\mathrm{~m}, 4 \mathrm{H}$, benzeneH), $7.19(\mathrm{~d}, 1 \mathrm{H}, J=8.5 \mathrm{~Hz}$, benzeneH), $7.14(\mathrm{~d}, 1 \mathrm{H}, J=$ $3.1 \mathrm{~Hz}$, pyrroleH), 7.07 (m, $2 \mathrm{H}$, benzeneH), 6.53 (dd, $1 \mathrm{H}, J=3.1,0.6 \mathrm{~Hz}$, pyrroleH), 5.31 (s, $\left.2 \mathrm{H}, \mathrm{CH}_{2}\right), 1.17(\mathrm{~m}, 21 \mathrm{H}, \mathrm{TIPS}) .{ }^{13} \mathrm{C} \mathrm{NMR}\left(101 \mathrm{MHz}, \mathrm{CDCl}_{3}\right) \delta 137.2,135.9,129.2,128.8$, $128.5,127.7,126.6,125.8,125.3,114.5,109.6,108.9,102.0,87.2,50.2,18.7,11.4$. IR 2958 
(m), 2942 (s), 2928 (m), 2892 (m), 2865 (s), 2148 (m), 1667 (w), 1512 (w), 1496 (w), 1480 (m), 1464 (m), 1457 (m), $1391(\mathrm{w}), 1356$ (w), $1331(\mathrm{w}), 1265$ (m), 1234 (w), 1215 (w), 1077 (w), 1016 (w), 998 (w), 946 (w), $884(\mathrm{~m}), 824$ (m), 803 (w), 777 (w), $761(\mathrm{~m}), 741$ (s), 731 (s). HRMS (ESI) calcd for $\mathrm{C}_{26} \mathrm{H}_{34} \mathrm{NSi}^{+}[\mathrm{M}+\mathrm{H}]^{+} 388.2455$; found 388.2460.

\section{1-(3-Methoxypropyl)-5-((triisopropylsilyl)ethynyl)-1H-indole (8d)}

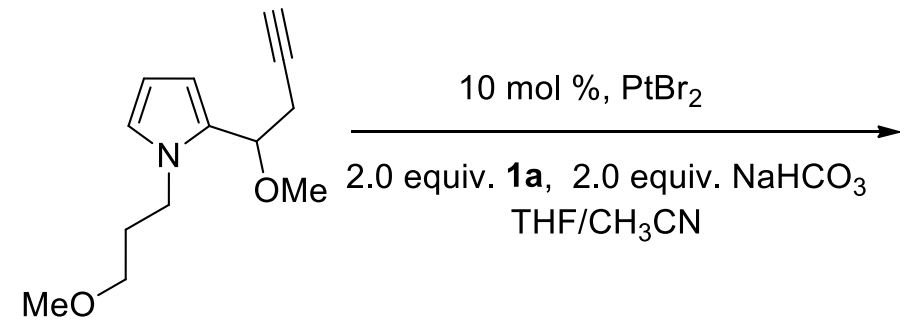

$6 d$

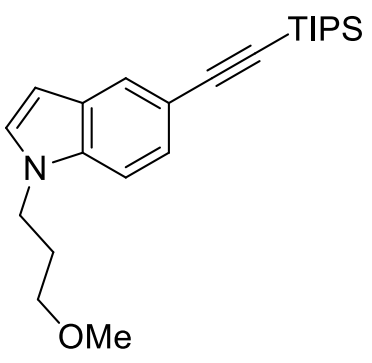

8d

Starting from 6d $(66 \mathrm{mg}, 0.30 \mathrm{mmol})$, the crude product was purified by column chromatography (Pentane/DCM=10:1) to afford $\mathbf{8 d}$ as yellow oil (89 $\mathrm{mg}, 0.24 \mathrm{mmol}, 80 \%)$.

Rf (Pentane / DCM= $10 /$ 1): 0.1. ${ }^{1} \mathrm{H}$ NMR (400 MHz, $\left.\mathrm{CDCl}_{3}\right) \delta 7.81(\mathrm{~d}, 1 \mathrm{H}, J=0.6 \mathrm{~Hz}$, benzeneH), $7.34(\mathrm{dd}, 1 \mathrm{H}, J=8.5 \mathrm{~Hz}, 1.5 \mathrm{~Hz}$, benzeneH), $7.28(\mathrm{~d}, 1 \mathrm{H}, J=8.5 \mathrm{~Hz}$, benzeneH), $7.10(\mathrm{~d}, 1 \mathrm{H}, J=3.1 \mathrm{~Hz}$, pyrroleH), $6.47(\mathrm{dd}, 1 \mathrm{H}, J=3.1 \mathrm{~Hz}, 0.7 \mathrm{~Hz}$, pyrroleH), $4.25(\mathrm{t}, 1 \mathrm{H}$, $\left.J=6.7 \mathrm{~Hz}, \mathrm{NCH}_{2}\right), 3.32\left(\mathrm{~s}, 3 \mathrm{H}, \mathrm{OCH}_{3}\right), 3.24\left(\mathrm{t}, 2 \mathrm{H}, J=5.8 \mathrm{~Hz}, \mathrm{CH}_{2} \mathrm{OCH}_{3}\right), 2.04(\mathrm{~m}, 2 \mathrm{H}$, $\left.\mathrm{CH}_{2}\right), 1.17$ (m, $\left.21 \mathrm{H}, \mathrm{TIPS}\right) .{ }^{13} \mathrm{C} \mathrm{NMR}\left(101 \mathrm{MHz}, \mathrm{CDCl}_{3}\right) \delta 135.7,128.9,128.2,125.5,125.3$, 114.1, 109.2, 109.0, 101.4, 87.0, 68.8, 58.6, 42.9, 30.3, 18.7, 11.4. IR 2942 (s), $2890(\mathrm{~m})$, 2865 (s), 2812 (w), 2147 (s), 1513 (w), 1482 (s), 1463 (m), 1392 (w), 1352 (w), 1332 (w), $1254(\mathrm{w}), 1237$ (w), 1207 (w), 1121 (s), 1076 (w), 1015 (w), 996 (w), $946(\mathrm{w}), 915$ (w), 884 (s), $821(\mathrm{~s}), 804(\mathrm{~m}), 763(\mathrm{w}), 722(\mathrm{~m})$. HRMS (ESI) calcd for $\mathrm{C}_{23} \mathrm{H}_{36} \mathrm{NOSi}^{+}[\mathrm{M}+\mathrm{H}]^{+}$ 370.2561 ; found 370.2552 .

\section{1-(4,4,4-Trifluorobutyl)-5-((triisopropylsilyl)ethynyl)-1H-indole (8e)}
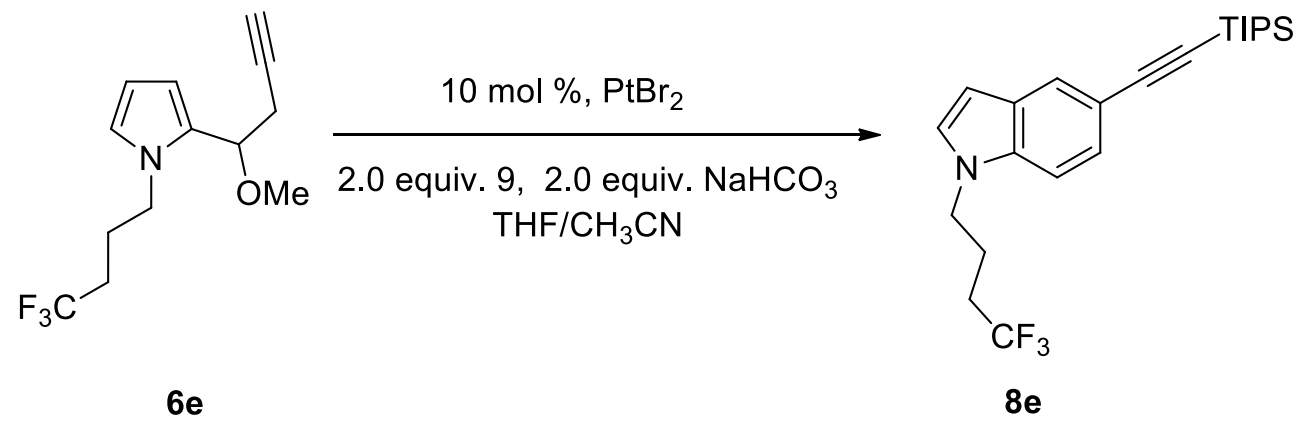

Starting from 6e $(83 \mathrm{mg}, 0.30 \mathrm{mmol})$, the crude product was purified by column chromatography (Pentane/DCM= 30:1) to afford 8e as yellow oil (100 mg, $0.250 \mathrm{mmol}, 82 \%)$.

Rf (Pentane / DCM= $30 / 1): 0.2 .{ }^{1} \mathrm{H}$ NMR (400 MHz, $\left.\mathrm{CDCl}_{3}\right) \delta 7.82(\mathrm{~m}, 1 \mathrm{H}$, benzeneH), $7.36(\mathrm{dd}, 1 \mathrm{H}, J=8.5,1.4 \mathrm{~Hz}$, benzeneH), $7.22(\mathrm{~d}, 1 \mathrm{H}, J=8.5 \mathrm{~Hz}$, benzeneH), $7.08(\mathrm{~d}, 1 \mathrm{H}, J$ $=3.1 \mathrm{~Hz}$, pyrroleH), $6.50(\mathrm{~d}, 1 \mathrm{H}, J=3.1 \mathrm{~Hz}$, pyrroleH $), 4.20\left(\mathrm{t}, 3 \mathrm{H}, J=6.5 \mathrm{~Hz}, \mathrm{NCH}_{2}\right), 2.07$ $\left(\mathrm{m}, 4 \mathrm{H}, \mathrm{CH}_{2} \mathrm{CH}_{2} \mathrm{CF}_{3}\right), 1.15(\mathrm{~m}, 21 \mathrm{H}, \mathrm{TIPS}) .{ }^{13} \mathrm{C} \mathrm{NMR}\left(101 \mathrm{MHz}, \mathrm{CDCl}_{3}\right) \delta 135.5,128.4$, $128.3,126.8(\mathrm{q}, J=275.8 \mathrm{~Hz}) 125.8,125.5,114.6,108.9,108.7,102.1,87.4,44.9,31.0(\mathrm{q}, J$ = 29.3 Hz), 22.9 (q, $J=2.9 \mathrm{~Hz}), 18.7$, 11.4. IR $2943(\mathrm{~m}), 2869(\mathrm{~m}), 2235(\mathrm{w}), 1687(\mathrm{w}), 1675$ 
(w), $1674(\mathrm{w}), 1617(\mathrm{w}), 1466(\mathrm{w}), 1466(\mathrm{w}), 1325(\mathrm{~s}), 1168(\mathrm{~m}), 1101(\mathrm{~s}), 1067(\mathrm{~s}), 1012(\mathrm{~m})$, $927(\mathrm{w}), 883(\mathrm{~m}), 840(\mathrm{~m}), 809$ (w), 735 (s). HRMS (ESI) calcd for $\mathrm{C}_{23} \mathrm{~F}_{3} \mathrm{H}_{33} \mathrm{NSi}^{+}[\mathrm{M}+\mathrm{H}]^{+}$ 408.2329; found 408.2338.

\section{1,4-Dimethyl-5-((triisopropylsilyl)ethynyl)-1H-indole (8f)}<smiles>CC#CCC(OC)c1cccn1C</smiles>

$6 f$

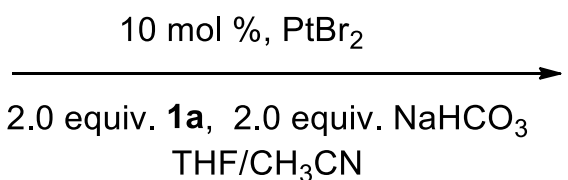
$\mathrm{THF} / \mathrm{CH}_{3} \mathrm{CN}$

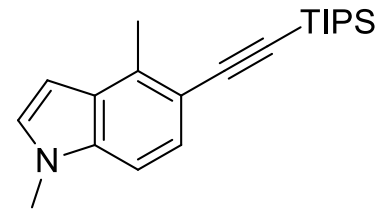

$8 f$

Starting from 6f $(53 \mathrm{mg}, 0.30 \mathrm{mmol})$, the crude product was purified by column chromatography (Pentane/DCM=20:1) to afford $8 \mathbf{f}$ as yellow oil (68 $\mathrm{mg}, 0.21 \mathrm{mmol}, 70 \%)$.

Rf (Pentane / DCM= $20 / 1): 0.4 .{ }^{1} \mathrm{H}$ NMR (400 MHz, $\left.\mathrm{CDCl}_{3}\right) \delta 7.36(\mathrm{~d}, 1 \mathrm{H}, J=8.5 \mathrm{~Hz}$, benzeneH), $7.10(\mathrm{~d}, 1 \mathrm{H}, J=8.5 \mathrm{~Hz}$, benzeneH), $7.05(\mathrm{~d}, 1 \mathrm{H}, J=3.1 \mathrm{~Hz}$, pyrroleH), $6.52(\mathrm{~d}$, $1 \mathrm{H}, J=3.1$, pyrroleH), $3.77\left(\mathrm{~s}, 3 \mathrm{H}, \mathrm{NCH}_{3}\right), 2.71\left(\mathrm{~s}, 3 \mathrm{H}, \mathrm{CH}_{3}\right), 1.19$ (s, $21 \mathrm{H}$, TIPS). ${ }^{13} \mathrm{C}$ NMR (101 MHz, $\left.\mathrm{CDCl}_{3}\right) \delta 136.0,133.9,128.9,128.3,126.2,113.5,107.6,106.7,100.2,91.2$, 32.9, 18.8, 17.2, 11.5. IR 2941 (s), 2925 (m), 2891 (m), 2863 (s), 2144 (s), 1517 (w), 1483 (m), 1463 (m), $1421(\mathrm{w}), 1379$ (w), 1366 (w), 1335 (w), 1283 (m), 1236 (w), 1152 (w), 1090 (w), $1071(\mathrm{w}), 1014(\mathrm{w}), 996$ (w), 911 (m), 882 (s), 816 (s). HRMS (ESI) calcd for $\mathrm{C}_{21} \mathrm{H}_{32} \mathrm{NSi}^{+}[\mathrm{M}+\mathrm{H}]^{+}$326.2299; found 326.2301.

\section{1-Methyl-4-phenyl-5-((triisopropylsilyl)ethynyl)-1H-indole (8g)}<smiles>COC(CC#Cc1ccccc1)c1cccn1C</smiles>

$6 \mathrm{~g}$

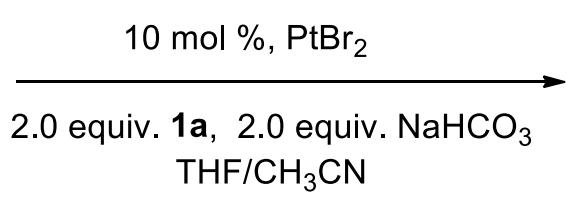

$$
\mathrm{THF} / \mathrm{CH}_{3} \mathrm{CN}
$$<smiles>Cn1ccc2c(-c3ccccc3)c(C#C[In]S)ccc21</smiles>

$8 \mathrm{~g}$

Starting from $\mathbf{6 g}(72 \mathrm{mg}, 0.30 \mathrm{mmol})$, the crude product was purified by column chromatography (Pentane/DCM= 50:1) to afford $\mathbf{8 g}$ as yellow oil $(65 \mathrm{mg}, 0.17 \mathrm{mmol}, 56 \%)$. The ratio of regioisomers $(\mathbf{8 g}: \mathbf{1 0 c}=4: 1)$ was determined on the ${ }^{1} \mathrm{H}$ NMR spectrum of the crude reaction mixture (Comparing two peaks at $\delta 6.51$ and $\delta 6.39$ ).

Rf (Pentane / DCM= 50 / 1): 0.5. ${ }^{1} \mathrm{H}$ NMR (400 MHz, $\left.\mathrm{CDCl}_{3}\right) \delta 7.53(\mathrm{~d}, 1 \mathrm{H}, J=8.2 \mathrm{~Hz}$, benzeneH), $7.38(\mathrm{~m}, 5 \mathrm{H}$, benzeneH $), 7.30(\mathrm{~d}, 1 \mathrm{H}, J=8.2 \mathrm{~Hz}$, benzeneH $), 6.97(\mathrm{~d}, 1 \mathrm{H}, J=$ $3.1 \mathrm{~Hz}$, pyrroleH), $6.49\left(\mathrm{~d}, 1 \mathrm{H}, J=3.1 \mathrm{~Hz}\right.$, benzeneH), $3.16\left(\mathrm{~s}, 3 \mathrm{H}, \mathrm{NCH}_{3}\right), 0.92(\mathrm{~m}, 21 \mathrm{H}$, TIPS). ${ }^{13} \mathrm{C}$ NMR $\left(101 \mathrm{MHz}, \mathrm{CDCl}_{3}\right) \delta 138.4,133.8,132.2,130.7,129.7,129.5,127.5,127.4$, 123.9, 119.8, 117.0, 107.5, 101.1, 92.0, 36.6, 18.6, 11.2. IR $2942(\mathrm{~m}), 2891(\mathrm{w}), 2864(\mathrm{~m})$, $2145(\mathrm{w}), 1519(\mathrm{w}), 1464(\mathrm{w}), 1314(\mathrm{w}), 1095(\mathrm{w}), 1017(\mathrm{w}), 997(\mathrm{w}), 962(\mathrm{w}), 884(\mathrm{w}), 814$ (w), 761 (w), $712(\mathrm{~m})$. HRMS (ESI) calcd for $\mathrm{C}_{26} \mathrm{H}_{33} \mathrm{NSi}[\mathrm{M}]^{+}$387.2382; found 387.2376. 


\section{1-Methyl-4-(p-tolyl)-5-((triisopropylsilyl)ethynyl)-1H-indole $(8 \mathrm{~h})$}<smiles>COC(CC#Cc1ccc(C)cc1)c1cccn1C</smiles>

$6 \mathrm{~h}$

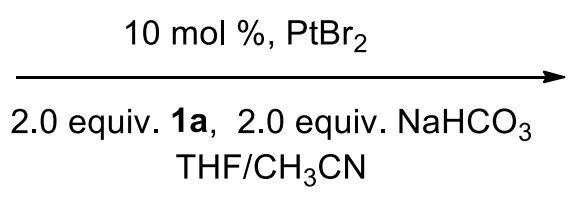

$\mathrm{THF} / \mathrm{CH}_{3} \mathrm{CN}$

Starting from $6 \mathrm{~h}(76 \mathrm{mg}, 0.30 \mathrm{mmol})$, the crude product was purified by column chromatography (Pentane/DCM=15:1) to afford $\mathbf{8 h}$ as yellow oil (63 $\mathrm{mg}, 0.16 \mathrm{mmol}, 52 \%)$. The ratio of regioisomeres $(\mathbf{8 h}: \mathbf{1 0 k}=3: 1)$ were determined by the spectra of crude product. The ratio of regioisomers was determined on the ${ }^{1} \mathrm{H}$ NMR spectrum of the crude reaction mixture (Comparing two peaks at $\delta 6.42$ and $\delta 6.30$ )..

Rf (Pentane / DCM= $15 / 1): 0.4 .{ }^{1} \mathrm{H}$ NMR (400 MHz, $\left.\mathrm{CDCl}_{3}\right) \delta 7.47$ (d, $1 \mathrm{H}, J=8.2 \mathrm{~Hz}$, benzeneH), $7.24(\mathrm{~m}, 3 \mathrm{H}$, benzeneH $), 7.14(\mathrm{~m}, 2 \mathrm{H}$, benzeneH $), 6.93(\mathrm{~d}, 1 \mathrm{H}, J=3.1 \mathrm{~Hz}$, pyrroleH), $6.44(\mathrm{~d}, 1 \mathrm{H}, J=3.1 \mathrm{~Hz}$, pyrrole $\mathrm{H}), 3.16\left(\mathrm{~s}, 3 \mathrm{H}, \mathrm{NCH}_{3}\right), 2.36$ (s, $\left.3 \mathrm{H}, \mathrm{CH}_{3}\right), 0.89$ $\left(\mathrm{m}, 21 \mathrm{H}\right.$, TIPS). ${ }^{13} \mathrm{C} \mathrm{NMR}\left(101 \mathrm{MHz}, \mathrm{CDCl}_{3}\right) \delta 136.9,135.4,133.9,132.1,130.5,129.8$, 129.5, 128.2, 123.8, 119.6, 117.2, 107.6, 101.1, 91.7, 36.6, 21.2, 18.5, 11.3. IR $3298(\mathrm{w})$, $2984(\mathrm{w}), 2938$ (m), 2868 (w), 2826 (w), 1713 (w), 1459 (w), 1105 (s), 1009 (w), 760 (m). HRMS (ESI) calcd for $\mathrm{C}_{27} \mathrm{H}_{36} \mathrm{NSi}^{+}[\mathrm{M}+\mathrm{H}]^{+}$402.2612; found 402.2605.

\section{4-(4-Bromophenyl)-1-methyl-5-((triisopropylsilyl)ethynyl)-1H-indole (8i)}<smiles>COC(CC#Cc1ccc(Br)cc1)c1cccn1C</smiles>

$6 \mathbf{i}$

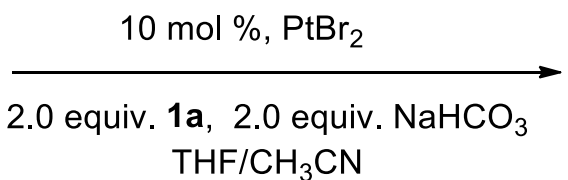
$\mathrm{THF} / \mathrm{CH}_{3} \mathrm{CN}$<smiles>Cn1ccc2c(-c3ccc(Br)cc3)c(C#C[In]S)ccc21</smiles>

8i

Starting from $\mathbf{6 i}(95 \mathrm{mg}, 0.30 \mathrm{mmol}$ ), the crude product was purified by column chromatography (Pentane/DCM=30:1) to afford 8i as yellow oil (62 $\mathrm{mg}, 0.14 \mathrm{mmol}, 44 \%$ ). The ratio of regioisomeres $(\mathbf{8 i : 1 0 1}=2.5: 1)$ was determined on the ${ }^{1} \mathrm{H}$ NMR spectrum of the crude reaction mixture (Comparing the two peaks at $\delta 6.49$ and $\delta 6.30$ ).

Rf (Pentane / DCM= $30 / 1): 0.3 .{ }^{1} \mathrm{H}$ NMR $\left(400 \mathrm{MHz}, \mathrm{CDCl}_{3}\right) \delta 7.53(\mathrm{~d}, 3 \mathrm{H}, J=8.2 \mathrm{~Hz}, \mathrm{Ar}$ H), 7.29 (dd, $3 \mathrm{H}, J=8.4,1.5 \mathrm{~Hz}, \operatorname{Ar~H}), 6.98(\mathrm{~d}, 1 \mathrm{H}, J=3.1 \mathrm{~Hz}, \operatorname{Ar~H}), 6.49$ (d, $1 \mathrm{H}, J=3.1$ $\mathrm{Hz}, \mathrm{Ar} \mathrm{H}), 3.21$ (s, $\left.3 \mathrm{H}, \mathrm{NCH}_{3}\right), 0.95$ (m, $\left.21 \mathrm{H}, \mathrm{TIPS}\right) .{ }^{13} \mathrm{C} \mathrm{NMR}\left(101 \mathrm{MHz}, \mathrm{CDCl}_{3}\right) \delta 137.6$, 
133.6, 132.4, 132.3, 130.8, 129.7, 128.1, 123.9, 121.8, 120.2, 117.1, 107.1, 101.3, 92.6, 36.8, 18.5, 11.2. IR 2945 (s), $2891(w), 2864$ (s), 2390 (w), 2358 (w), 2342 (w), 2297 (w), 2143 (w), $1516(\mathrm{w}), 1464$ (m), 1316 (w), 1097 (w), 1072 (w), 1016 (w), 963 (w), 915 (w), $885(\mathrm{w}), 819$ (s), 730 (s), 717 (m). HRMS (ESI) calcd for $\mathrm{C}_{26} \mathrm{H}_{33} \mathrm{BrNSi}^{+}[\mathrm{M}+\mathrm{H}]^{+}$466.1560; found 466.1556 .

\section{1-Methyl-4-(naphthalen-1-yl)-5-((triisopropylsilyl)ethynyl)-1H-indole (8j)}<smiles>COC(CC#Cc1cccc2ccccc12)c1cccn1C</smiles>

6j

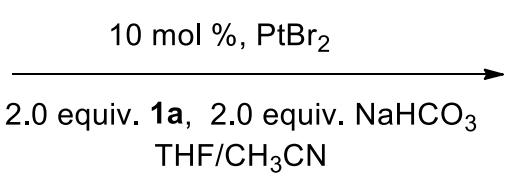

8j<smiles>Cn1ccc2c(-c3cccc4ccccc34)c(C#C[In]S)ccc21</smiles><smiles>CC(F)C#Cc1ccc2ccn(C)c2c1-c1cccc2ccccc12</smiles>

$10 d$

Starting from $\mathbf{6 i}(87 \mathrm{mg}, 0.30 \mathrm{mmol})$, the crude product was purified by column chromatography (Pentane/DCM=10:1) to afford $\mathbf{8 j}$ as yellow oil (41 mg, $0.094 \mathrm{mmol}, 31 \%$ ) and 10d as yellow oil (41 mg, $0.094 \mathrm{mmol}, 31 \%)$.

\section{$8 \mathbf{j}$}

Rf (Pentane / DCM= 15 / 1): 0.6. ${ }^{1} \mathrm{H}$ NMR (400 MHz, $\left.\mathrm{CDCl}_{3}\right) \delta$ 7.78-7.74 (m, $2 \mathrm{H}$, benzeneH $)$, 7.44-7.38 (m, $4 \mathrm{H}$, benzeneH $), 7.31(\mathrm{~m}, 1 \mathrm{H}$, benzeneH $), 7.24-7.18(\mathrm{~m}, 2 \mathrm{H}$, benzeneH), $6.88(\mathrm{~d}, 1 \mathrm{H}, J=3.1 \mathrm{~Hz}$, pyrroleH $), 5.88(\mathrm{~d}, 1 \mathrm{H}, J=3.1 \mathrm{~Hz}$, pyrroleH $), 3.74(\mathrm{~s}, 3$ $\left.\mathrm{H}, \mathrm{NCH}_{3}\right), 0.65-0.60(\mathrm{~m}, 21 \mathrm{H}, \mathrm{TIPS}) .{ }^{13} \mathrm{C} \mathrm{NMR}\left(101 \mathrm{MHz}, \mathrm{CDCl}_{3}\right) \delta 137.4,136.8,136.1$, 133.7, 132.2, 129.5, 129.1, 127.9, 127.5, 127.4, 126.6, 126.3, 125.5, 125.3, 125.2, 114.6, 108.4, 107.3, 101.5, 90.9, 33.0, 18.3, 11.0. IR 2944 (m), 2892 (w), 2891 (w), 2865 (w), 2864 (w), 2362 (s), 2361 (s), 2148 (w), 1465 (w), 911 (w), 887 (w), 886 (w), 820 (w), 800 (w), 777 (w), $734(\mathrm{~m})$. HRMS (ESI) calcd for $\mathrm{C}_{30} \mathrm{H}_{35} \mathrm{NSi}^{+}[\mathrm{M}]^{+}$438.2612; found 438.2605.

\section{0d}

Rf (Pentane / DCM= 15 / 1): 0.5. ${ }^{1} \mathrm{H}$ NMR (400 MHz, $\left.\mathrm{CDCl}_{3}\right) \delta$ 7.89-7.86 (m, $2 \mathrm{H}$, benzeneH), $7.62(\mathrm{~d}, 1 \mathrm{H}, J=8.2 \mathrm{~Hz}$, benzeneH $), 7.53-7.49(\mathrm{~m}, 2 \mathrm{H}$, benzeneH $), 7.45-7.30$ $(\mathrm{m}, 4 \mathrm{H}$, benzeneH $), 6.93(\mathrm{~d}, 1 \mathrm{H}, J=3.1 \mathrm{~Hz}$, pyrroleH $), 6.53(\mathrm{~d}, 1 \mathrm{H}, J=3.1 \mathrm{~Hz}$, pyrroleH), $2.88\left(\mathrm{~s}, 3 \mathrm{H}, \mathrm{NCH}_{3}\right), 0.70(\mathrm{~m}, 21 \mathrm{H}, \mathrm{TIPS}) .{ }^{13} \mathrm{C} \mathrm{NMR}\left(101 \mathrm{MHz}, \mathrm{CDCl}_{3}\right) \delta 136.1$, 134.6, 133.6, 133.4, 132.0, 129.5, 128.4, 128.0, 127.9, 127.5, 126.6, 126.1, 125.7, 124.9, 123.9, 120.1, 117.8, 107.2, 101.1, 91.8, 35.6, 18.3, 11.0. IR $3055(\mathrm{w}), 2942(\mathrm{~s}), 2892(\mathrm{~m})$, 2863 (s), 2145 (m), 1514 (w), $1464(\mathrm{~m}), 1410(\mathrm{w}), 1409$ (w), $1387(\mathrm{w}), 1314(\mathrm{w}), 1234(\mathrm{w})$, 1082 (w), 1016 (w), 996 (w), 956 (m), 911 (m), $884(\mathrm{~m}), 804$ (s), 778 (m), 732 (s), 716 (s). HRMS (ESI) calcd for $\mathrm{C}_{30} \mathrm{H}_{35} \mathrm{NSi}^{+}[\mathrm{M}]^{+} 438.2612$; found 438.2608 .

\section{1,6-Dimethyl-5-((triisopropylsilyl)ethynyl)-1H-indole (8k)}


<smiles>C#CC(C)C(OC)c1cccn1C</smiles>

$6 \mathrm{k}$

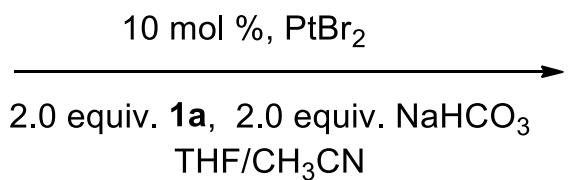

$\mathrm{THF} / \mathrm{CH}_{3} \mathrm{CN}$

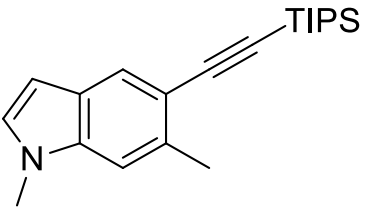

$8 \mathbf{k}$

Starting from 6k $(52 \mathrm{mg}, 0.30 \mathrm{mmol})$, the crude product was purified by column chromatography (Pentane/DCM= 15:1) to afford $\mathbf{8 k}$ as yellow oil (73 $\mathrm{mg}, 0.22 \mathrm{mmol}$, $75 \%)^{[10]}$

Rf (Pentane / DCM= 15 / 1): 0.4. ${ }^{1} \mathrm{H}$ NMR (400 MHz, $\left.\mathrm{CDCl}_{3}\right) \delta 7.79(\mathrm{~s}, 1 \mathrm{H}$, benzeneH), 7.13 (s, $1 \mathrm{H}$, benzeneH), $6.98(\mathrm{~d}, 1 \mathrm{H}, J=3.1 \mathrm{~Hz}$, pyrroleH), $6.40(\mathrm{dd}, 1 \mathrm{H}, J=3.1,0.7 \mathrm{~Hz}$, pyrroleH), $3.74\left(\mathrm{~s}, 3 \mathrm{H}, \mathrm{NCH}_{3}\right), 2.60\left(\mathrm{~s}, 3 \mathrm{H}, \mathrm{CH}_{3}\right), 1.16(\mathrm{~m}, 21 \mathrm{H}, \mathrm{TIPS}) .{ }^{13} \mathrm{C} \mathrm{NMR}(101$ $\left.\mathrm{MHz} \mathrm{CDCl}_{3}\right) \delta 136.8,133.7,129.1,126.3,125.5,114.9,109.5,107.6,100.9,91.0,32.8,21.6$, 18.8, 11.5. IR 2943 (s), $2893(\mathrm{~m}), 2865$ (s), 2143 (s), $1622(\mathrm{w}), 1514(\mathrm{w}), 1477(\mathrm{~m}), 1425(\mathrm{w})$, $1377(\mathrm{w}), 1326(\mathrm{w}), 1271(\mathrm{w}), 1238(\mathrm{w}), 1076(\mathrm{w}), 1013(\mathrm{w}), 997(\mathrm{w}), 913(\mathrm{w}), 885(\mathrm{~m}), 845$ (m), $818(\mathrm{w}), 741(\mathrm{w}), 720(\mathrm{~m})$. HRMS (ESI) calcd for $\mathrm{C}_{21} \mathrm{H}_{32} \mathrm{NSi}^{+}[\mathrm{M}+\mathrm{H}]^{+}$326.2299; found 326.2303 .

\section{1,4-Dimethyl-6-((triisopropylsilyl)ethynyl)-1H-indole (10e)}<smiles>C#CCC(C)(OC)c1cccn1C</smiles>

6l

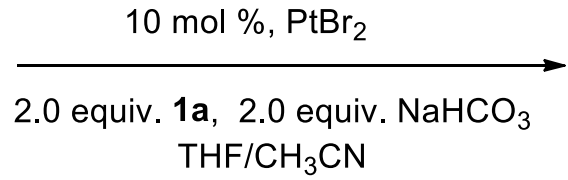

$\mathrm{THF} / \mathrm{CH}_{3} \mathrm{CN}$

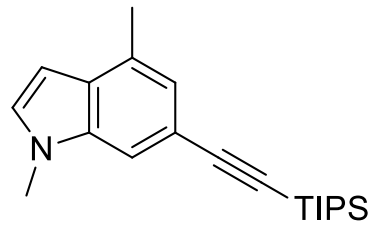

$10 \mathrm{e}$

Starting from 61 (52 $\mathrm{mg}, 0.30 \mathrm{mmol})$, the crude product was purified by column chromatography (Pentane/DCM= 20:1) to afford 10e as yellow oil (53 mg, $0.16 \mathrm{mmol}, 54 \%)$.

Rf (Pentane / DCM= 20 / 1): 0.5. ${ }^{1} \mathrm{H}$ NMR (400 MHz, $\left.\mathrm{CDCl}_{3}\right) \delta 7.33$ (s, $1 \mathrm{H}$, benzeneH), 7.08 $(\mathrm{d}, 1 \mathrm{H}, J=3.1 \mathrm{~Hz}$, pyrroleH), $7.04(\mathrm{~s}, 1 \mathrm{H}$, benzeneH), $6.46(\mathrm{dd}, 1 \mathrm{H}, J=3.1,0.8 \mathrm{~Hz}$, pyrroleH), $3.78\left(\mathrm{~s}, 3 \mathrm{H}, \mathrm{NCH}_{3}\right), 2.51\left(\mathrm{~s}, 3 \mathrm{H}, \mathrm{CH}_{3}\right), 1.16(\mathrm{~s}, 21 \mathrm{H}, \mathrm{TIPS}) .{ }^{13} \mathrm{C}$ NMR $(101 \mathrm{MHz}$, $\left.\mathrm{CDCl}_{3}\right) \delta 135.9,130.1,129.6,128.7,123.4,116.2,110.9,109.2,99.8,87.8,33.0,18.8,18.5$, 11.5. IR 2957 (w), 2942 (m), 2927 (w), 2889 (w), 2865 (m), $2144(\mathrm{w}), 1510(\mathrm{w}), 1463(\mathrm{w})$, $1299(\mathrm{w}), 884(\mathrm{w}), 767(\mathrm{w}), 740$ (w), $710(\mathrm{~m}), 682(\mathrm{~m}), 671(\mathrm{~m}), 659(\mathrm{w}), 648(\mathrm{w}), 637(\mathrm{~s})$, 617 (w). HRMS (ESI) calcd for $\mathrm{C}_{21} \mathrm{H}_{32} \mathrm{NSi}^{+}[\mathrm{M}+\mathrm{H}]^{+}$326.2299; found 326.2302.

\section{9-Methyl-3-((triisopropylsilyl)ethynyl)-9H-carbazole (8m)}<smiles>C#CCC(OC)c1cc2ccccc2n1C</smiles>

$6 m$

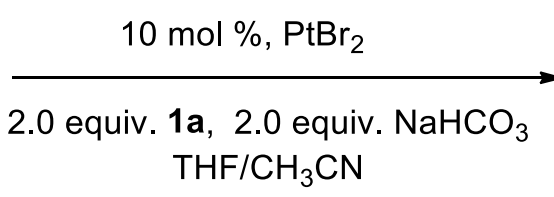

$\mathrm{THF} / \mathrm{CH}_{3} \mathrm{CN}$<smiles>Cn1c2ccccc2c2cc(C#C[TlH]S)ccc21</smiles>

$8 m$

[10] The product contains about $5 \%$ impurity. 
Starting from $6 \mathrm{~m}(64 \mathrm{mg}, 0.30 \mathrm{mmol})$, the crude product was purified by column chromatography (Pentane/DCM= 50:1) to afford $\mathbf{8 m}$ as yellow oil ( $82 \mathrm{mg}, 0.23 \mathrm{mmol}, 76 \%$ ).

Rf (Pentane / DCM= $50 / 1): 0.3 .{ }^{1} \mathrm{H}$ NMR (400 MHz, $\left.\mathrm{CDCl}_{3}\right) \delta 8.25(\mathrm{~m}, 1 \mathrm{H}$, benzeneH), $8.10(\mathrm{~d}, 1 \mathrm{H}, J=7.8 \mathrm{~Hz}$, benzeneH), $7.62(\mathrm{dd}, 1 \mathrm{H}, J=1.5,8.4 \mathrm{~Hz}$, benzeneH), $7.51(\mathrm{~m}, 1 \mathrm{H}$, benzeneH), $7.40(\mathrm{~m}, 1 \mathrm{H}$, benzeneH), 7.31-7.28 (m, $2 \mathrm{H}$, benzeneH $), 3.83\left(\mathrm{~s}, 3 \mathrm{H}, \mathrm{NCH}_{3}\right), 1.22$ (s, $21 \mathrm{H}$, TIPS). ${ }^{13} \mathrm{C}$ NMR $\left(101 \mathrm{MHz}, \mathrm{CDCl}_{3}\right) \delta 141.3,140.5,129.8,126.1,124.4,122.6$, 122.3, 120.5, 119.3, 113.6, 108.7, 108.6, 108.2, 87.7, 29.1, 18.8, 11.5. IR $2944(\mathrm{w}), 2891(\mathrm{w})$, $2890(\mathrm{w}), 2866$ (w), 2865 (w), 2148 (w), 2147 (w), 1474 (w), 1249 (w), 907 (s), 730 (s). HRMS (ESI) calcd for $\mathrm{C}_{24} \mathrm{H}_{32} \mathrm{NSi}^{+}[\mathrm{M}]^{+}$362.2299; found 362.2291.

\section{4-Methyl-7-((triisopropylsilyl)ethynyl)-4H-thieno[3,2-b]indole (8n)}<smiles>C#CCC(OC)c1cc2sccc2n1C</smiles>

$6 n$

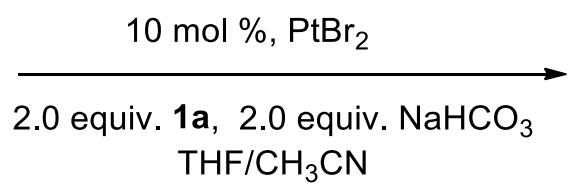

$\mathrm{THF} / \mathrm{CH}_{3} \mathrm{CN}$

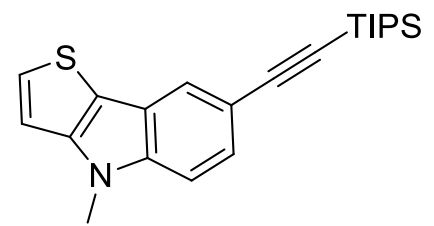

$8 n$

Starting from 6n $(66 \mathrm{mg}, 0.30 \mathrm{mmol})$, the crude product was purified by column chromatography (Pentane/DCM= 20:1) to afford $\mathbf{8 n}$ as yellow oil ( $86 \mathrm{mg}, 0.23 \mathrm{mmol}, 78 \%$ ).

Rf (Pentane / DCM= 20 / 1): 0.4. ${ }^{1} \mathrm{H}$ NMR (400 MHz, $\left.\mathrm{CDCl}_{3}\right) \delta 7.92$ (dd, $1 \mathrm{H}, J=1.4,0.4 \mathrm{~Hz}$, benzeneH), $7.43(\mathrm{dd}, 1 \mathrm{H}, J=8.5,1.5 \mathrm{~Hz}$, benzeneH $), 7.40(\mathrm{~d}, 1 \mathrm{H}, J=5.2 \mathrm{~Hz}$, thiophenH), $7.28(\mathrm{dd}, 1 \mathrm{H}, J=8.5,0.4 \mathrm{~Hz}$, benzeneH), $7.06(\mathrm{~d}, 1 \mathrm{H}, J=5.2 \mathrm{~Hz}$, thiophenH), $3.87(\mathrm{~s}, 3 \mathrm{H}$, $\left.\mathrm{NCH}_{3}\right), 1.17$ (s, $\left.21 \mathrm{H}, \mathrm{TIPS}\right) .{ }^{13} \mathrm{C} \mathrm{NMR}\left(101 \mathrm{MHz}, \mathrm{CDCl}_{3}\right) \delta 146.5,141.5,127.4,126.5,123.0$, 121.4, 115.8, 113.9, 110.0, 109.4, 108.6, 87.7, 31.3, 18.8, 11.5. IR 3298 (w), 2979 (w), 2934 (w), $2914(\mathrm{w}), 1509(\mathrm{w}), 1422(\mathrm{w}), 1374(\mathrm{w}), 1256(\mathrm{w}), 1177(\mathrm{w}), 936(\mathrm{w}), 786(\mathrm{~m}), 738(\mathrm{w})$, $706(\mathrm{~m}), 695(\mathrm{~m}), 681(\mathrm{~m}), 653(\mathrm{~m}), 646(\mathrm{~s}), 623(\mathrm{~m}), 607(\mathrm{~m})$. HRMS (ESI) calcd for $\mathrm{C}_{22} \mathrm{H}_{30} \mathrm{NSSi}^{+}[\mathrm{M}+\mathrm{H}]^{+}$368.1863; found 368.1861.

\section{1-Methyl-6-((triisopropylsilyl)ethynyl)-1H-indole (10a)}

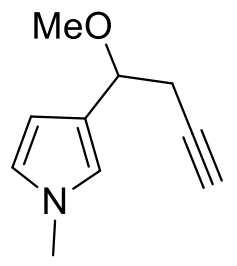

$9 a$

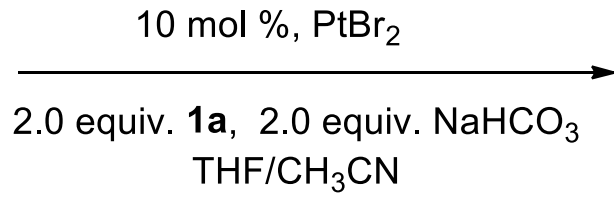

$\mathrm{THF} / \mathrm{CH}_{3} \mathrm{CN}$

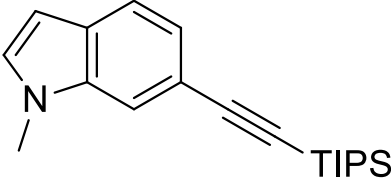

$10 a$

Starting from 9a $(49 \mathrm{mg}, 0.30 \mathrm{mmol})$, the crude product was purified by column chromatography (Pentane/DCM= 20:1) to afford 10a as yellow oil (72 mg, $0.23 \mathrm{mmol}, 77 \%)$.

Rf (Pentane / DCM= $20 /$ 1): 0.4. ${ }^{1} \mathrm{H}$ NMR (400 MHz, $\left.\mathrm{CDCl}_{3}\right) \delta 7.56(\mathrm{~d}, 1 \mathrm{H}, J=8.2$, benzeneH), $7.49(\mathrm{~s}, 1 \mathrm{H}$, benzeneH), $7.26(\mathrm{dd}, 2 \mathrm{H}, J=8.2,1.2 \mathrm{~Hz}$, benzeneH), $7.09(\mathrm{~d}, 1 \mathrm{H}, J$ $=3.0 \mathrm{~Hz}$, pyrroleH $), 6.50(\mathrm{~d}, 1 \mathrm{H}, J=3.0 \mathrm{~Hz}$, pyrroleH $), 3.79\left(\mathrm{~s}, 3 \mathrm{H}, N_{C H}\right), 1.17(\mathrm{~m}, 21 \mathrm{H}$, TIPS). ${ }^{13} \mathrm{C}$ NMR $\left(101 \mathrm{MHz}, \mathrm{CDCl}_{3}\right) \delta 136.1,130.2,128.6,123.5,120.6,116.1,113.1,109.0$, 101.2, 88.2, 32.9, 18.7, 11.4. IR 2943 (s), 2893 (m), 2865 (s), 2151 (m), 1511 (w), 1466 (s), 
$1421(\mathrm{w}), 1344$ (w), 1245 (w), 1076 (w), 1012 (w), 944 (m), 885 (m), 857 (w), 816 (m), 763 (w), 714 (s). HRMS (ESI) calcd for $\mathrm{C}_{20} \mathrm{H}_{30} \mathrm{NSi}^{+}[\mathrm{M}+\mathrm{H}]^{+} 312.2142$; found 312.2141.

\section{1,7-Dimethyl-6-((triisopropylsilyl)ethynyl)-1H-indole (10b)}

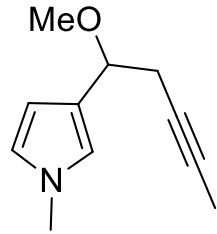

$9 b$

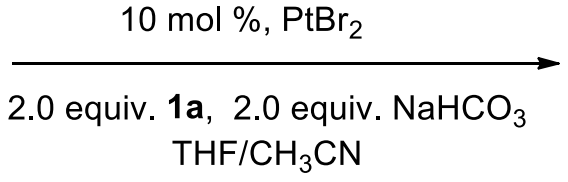

$\mathrm{THF} / \mathrm{CH}_{3} \mathrm{CN}$

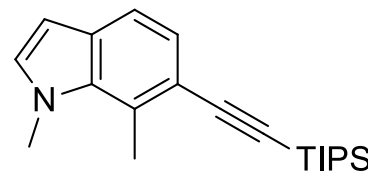

$10 \mathrm{~b}$

Starting from 9b $(52 \mathrm{mg}, 0.30 \mathrm{mmol})$, the crude product was purified by column chromatography (Pentane/DCM= 20:1) to afford $\mathbf{1 0 b}$ as yellow oil (48 mg, $0.15 \mathrm{mmol}, 49 \%$ ).

Rf (Pentane / DCM= $50 / 1): 0.3 .{ }^{1} \mathrm{H}$ NMR (400 MHz, $\left.\mathrm{CDCl}_{3}\right) \delta 7.35(\mathrm{~d}, 1 \mathrm{H}, J=8.2 \mathrm{~Hz}$, benzeneH), $7.20(\mathrm{~d}, 1 \mathrm{H}, J=8.2 \mathrm{~Hz}$, benzeneH), $6.97(\mathrm{~d}, 1 \mathrm{H}, J=3.1 \mathrm{~Hz}$, pyrroleH), 6.39 (d, $1 \mathrm{H}, \mathrm{J}=3.1 \mathrm{~Hz}$, pyrroleH), $4.06\left(\mathrm{~s}, 3 \mathrm{H}, \mathrm{NCH}_{3}\right), 2.95$ (s, $\left.3 \mathrm{H}, \mathrm{CH}_{3}\right), 1.16\left(\mathrm{~s}, 21 \mathrm{H}\right.$, TIPS). ${ }^{13} \mathrm{C}$ NMR $\left(101 \mathrm{MHz}, \mathrm{CDCl}_{3}\right) \delta 135.1,132.1,129.8,124.5,124.4,118.5,117.2,107.8,101.2,92.5$, 37.4, 18.8, 16.9, 11.5. IR 2942 (s), 2892 (m), 2865 (s), 2147 (m), 1515 (w), 1464 (m), 1313 (w), $1312(\mathrm{w}), 1001(\mathrm{w}), 911(\mathrm{~m}), 887(\mathrm{~m}), 886(\mathrm{~m}), 813(\mathrm{~m}), 730(\mathrm{~s})$. HRMS (ESI) calcd for $\mathrm{C}_{21} \mathrm{H}_{32} \mathrm{NSi}^{+}[\mathrm{M}]^{+}$326.2299; found 326.2295.

\section{1-Methyl-7-phenyl-6-((triisopropylsilyl)ethynyl)-1H-indole (10c)}

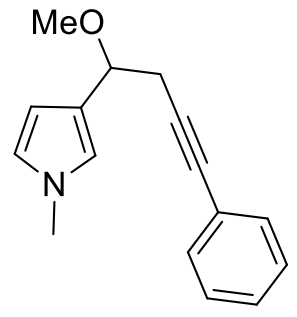

9c

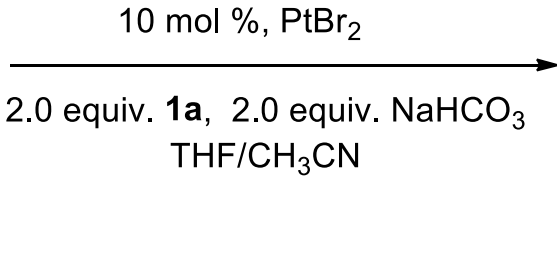

$\mathrm{THF} / \mathrm{CH}_{3} \mathrm{CN}$<smiles>Cn1ccc2ccc(C#CC(F)F)c(-c3ccccc3)c21</smiles>

$10 c$

Starting from 9c $(72 \mathrm{mg}, 0.30 \mathrm{mmol})$, the crude product was purified by column chromatography (Pentane/DCM= 20:1) to afford 10c as yellow oil $(62 \mathrm{mg}, 0.16 \mathrm{mmol}, 53 \%)$.

${ }^{1} \mathrm{H}$ NMR (400 MHz, $\left.\mathrm{CDCl}_{3}\right) \delta 7.53(\mathrm{~d}, 1 \mathrm{H}, J=8.2 \mathrm{~Hz}$, benzeneH), 7.42-7.35 (m, $5 \mathrm{H}$, benzeneH), 7.31 (d, $1 \mathrm{H}, J=8.2 \mathrm{~Hz}$, benzeneH), 6.97 (d, $1 \mathrm{H}, J=3.1 \mathrm{~Hz}$, pyrroleH), 6.49 (d, $1 \mathrm{H}, J=3.1 \mathrm{~Hz}$, pyrroleH), $3.16\left(\mathrm{~s}, 3 \mathrm{H}, \mathrm{NCH}_{3}\right), 0.93$ (m, $\left.21 \mathrm{H}, T I P S\right) .{ }^{13} \mathrm{C}$ NMR $(101 \mathrm{MHz}$, $\left.\mathrm{CDCl}_{3}\right) \delta 138.4,133.8,132.2,130.7,129.7,129.5,127.5,127.4,123.9,119.8,117.0,107.5$, 101.1, 92.0, 36.6, 18.6, 11.2. IR 2946 (w), 2866 (w), 2146 (w), 1465 (w), 1295 (w), $1273(\mathrm{w})$, $1203(\mathrm{~m}), 965$ (m), 906 (w), 883 (w), 814 (w), 763 (w), 712 (w). HRMS (ESI) calcd for $\mathrm{C}_{26} \mathrm{H}_{33} \mathrm{NSi}^{+}[\mathrm{M}]^{+}$388.2455; found 388.2457.

1-Methyl-7-(naphthalen-1-yl)-6-((triisopropylsilyl)ethynyl)-1H-indole (10d) 


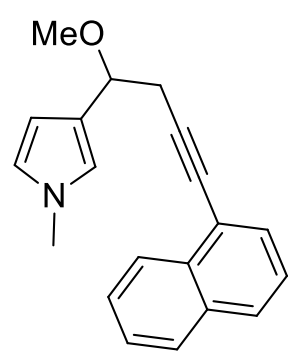

9d

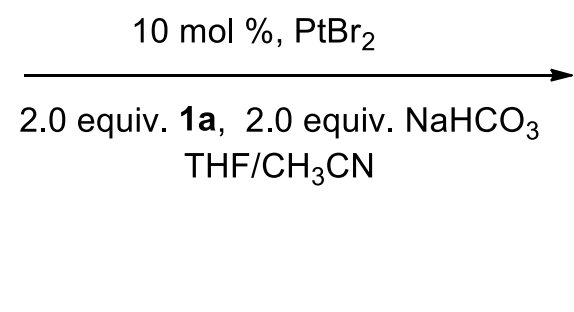

1a, 2.0 equiv. $\mathrm{NaHCO}_{3}$

$\mathrm{HF} / \mathrm{CH}_{3} \mathrm{CN}$

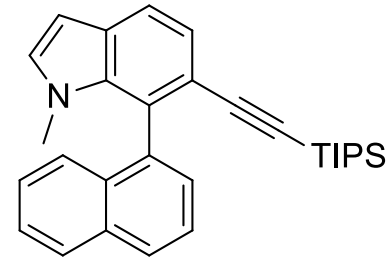

$10 d$

Starting from 9d $(87 \mathrm{mg}, 0.30 \mathrm{mmol})$, the crude product was purified by column chromatography (Pentane/DCM= 20:1) to afford 10d as yellow oil (62 mg, $0.14 \mathrm{mmol}, 47 \%)$.

Charactization datas corresonds to the one obtained starting from $\mathbf{6 i}$.

\section{1,4-Dimethyl-6-((triisopropylsilyl)ethynyl)-1H-indole (10e)}

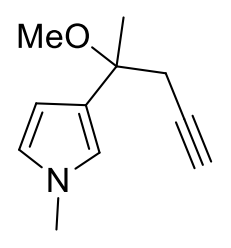

$9 e$

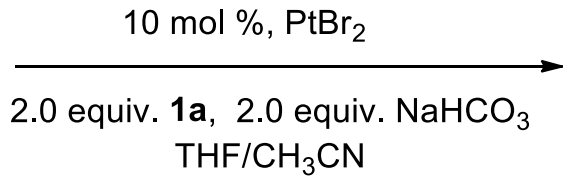

$\mathrm{THF} / \mathrm{CH}_{3} \mathrm{CN}$

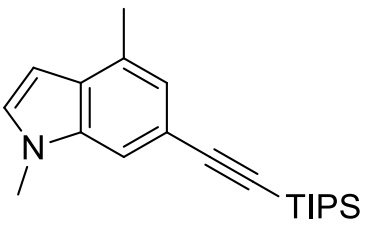

$10 \mathrm{e}$

Starting from 9e $(52 \mathrm{mg}, 0.30 \mathrm{mmol})$, the crude product was purified by column chromatography (Pentane/DCM= 20:1) to afford 10e as yellow oil (53 mg, $0.16 \mathrm{mmol}, 54 \%)$.

Charactization datas corresponds to the one obtained starting from $\mathbf{6 k}$.

\section{1-Methyl-4-phenyl-6-((triisopropylsilyl)ethynyl)-1H-indole (10f)}<smiles>C#CCC(C)(c1ccccc1)c1ccn(C)c1</smiles>

$9 f$

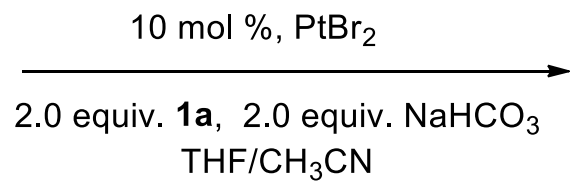

$\mathrm{THF} / \mathrm{CH}_{3} \mathrm{CN}$

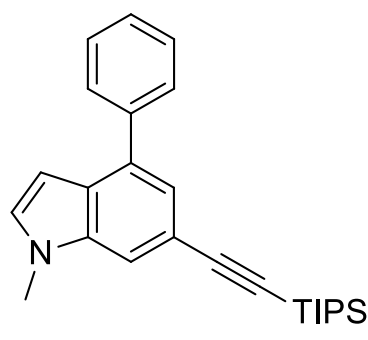

$10 f$

Starting from 9f $(72 \mathrm{mg}, 0.30 \mathrm{mmol})$, the crude product was purified by column chromatography (Pentane/DCM=15:1) to afford 10f as yellow oil (75 mg, $0.19 \mathrm{mmol}, 65 \%)$.

Rf (Pentane / DCM= 15 / 1): 0.4. ${ }^{1} \mathrm{H}$ NMR (400 MHz, $\left.\mathrm{CDCl}_{3}\right) \delta 7.70(\mathrm{dd}, 2 \mathrm{H}, J=8.4,1.2 \mathrm{~Hz}$, benzeneH), $7.49(\mathrm{~m}, 3 \mathrm{H}$, benzeneH $), 7.39(\mathrm{~m}, 1 \mathrm{H}$, benzeneH $), 7.33(\mathrm{~d}, 1 \mathrm{H}, J=1.2 \mathrm{~Hz}$, benzeneH), $7.14(\mathrm{~d}, 1 \mathrm{H}, J=3.1 \mathrm{~Hz}$, pyrroleH), $6.62(\mathrm{dd}, 1 \mathrm{H}, J=3.1,0.8 \mathrm{~Hz}$, pyrroleH), 3.83 $\left(\mathrm{s}, 3 \mathrm{H}, \mathrm{NCH}_{3}\right), 1.18$ (s, $\left.21 \mathrm{H}, \mathrm{TIPS}\right) .{ }^{13} \mathrm{C} \mathrm{NMR}\left(101 \mathrm{MHz}, \mathrm{CDCl}_{3}\right) \delta 140.5,136.7,134.4$, $130.5,128.7,128.5,127.2,127.0,123.3,116.5,112.2,108.8,100.9,88.4,33.1,18.8,11.5$. IR 2942 (s), 2891 (w), 2864 (s), 2148 (m), 1511 (m), 1499 (w), 1462 (m), $1431(\mathrm{w}), 1327(\mathrm{w})$, 1295 (w), 1264 (w), $1212(\mathrm{w}), 1091(\mathrm{w}), 1030(\mathrm{w}), 1017(\mathrm{w}), 971(\mathrm{~m}), 920(\mathrm{w}), 883(\mathrm{~m}), 850$ $(\mathrm{w}), 781(\mathrm{~m}), 761(\mathrm{w}), 729(\mathrm{w}), 711(\mathrm{~m})$. HRMS (ESI) calcd for $\mathrm{C}_{26} \mathrm{H}_{34} \mathrm{NSi}^{+}[\mathrm{M}+\mathrm{H}]^{+}$ 388.2455; found 388.2466. 


\section{1,4,7-Trimethyl-6-((triisopropylsilyl)ethynyl)-1H-indole (10g)}<smiles>CC#CCC(C)(OC)c1ccn(C)c1</smiles>

$9 \mathrm{~g}$

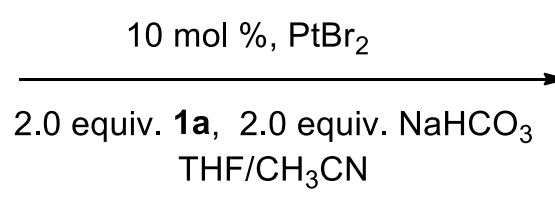

$\mathrm{THF} / \mathrm{CH}_{3} \mathrm{CN}$<smiles>Cc1cc(C#C[In])c(C)c2c1ccn2C</smiles>

$10 \mathrm{~g}$

Starting from 9g $(57 \mathrm{mg}, 0.30 \mathrm{mmol})$, the crude product was purified by column chromatography (Pentane/DCM= 20:1) to afford $\mathbf{1 0 g}$ as yellow oil (45 mg, $0.13 \mathrm{mmol}, 44 \%$ ).

Rf (Pentane / DCM= $20 /$ 1): 0.2. ${ }^{1} \mathrm{H}$ NMR (400 MHz, $\left.\mathrm{CDCl}_{3}\right) \delta 7.03(\mathrm{~s}, 1 \mathrm{H}$, benzeneH), 6.97 $\left(\mathrm{d}, 1 \mathrm{H}, J=3.1 \mathrm{~Hz}\right.$, pyrroleH), $6.41\left(\mathrm{~d}, 1 \mathrm{H}, J=3.1 \mathrm{~Hz}\right.$, pyrroleH), 4.05 (s, $\left.3 \mathrm{H}, \mathrm{NCH}_{3}\right), 2.92$ (s, $\left.3 \mathrm{H}, \mathrm{CH}_{3}\right), 2.46\left(\mathrm{~s}, 3 \mathrm{H}, \mathrm{CH}_{3}\right), 1.17(\mathrm{~s}, 21 \mathrm{H}, \mathrm{TIPS}) .{ }^{13} \mathrm{C} \mathrm{NMR}\left(101 \mathrm{MHz}, \mathrm{CDCl}_{3}\right) \delta 134.8$, 131.5, 129.9, 127.6, 124.1, 122.0, 117.0, 108.0, 99.6, 92.1, 37.3, 18.8, 18.1, 16.6, 11.5. IR 2958 (s), 2943 (s), 2926 (s), 2892 (m), 2865 (s), 2142 (s), 1517 (w), 1496 (w), 1463 (m), 1384 (w), 1346 (w), 1303 (w), $1091(\mathrm{w}), 1065$ (w), 1008 (w), 921 (w), 885 (s), 864 (w), 772 (w), 744 (w), 709 (s). HRMS (ESI) calcd for $\mathrm{C}_{22} \mathrm{H}_{34} \mathrm{NSS}^{+}[\mathrm{M}+\mathrm{H}]^{+}$340.2455; found 340.2460.

\section{1,4,5-Trimethyl-6-((triisopropylsilyl)ethynyl)-1H-indole (10h)}<smiles>C#CC(C)C(C)(OC)c1ccn(C)c1</smiles>

9h

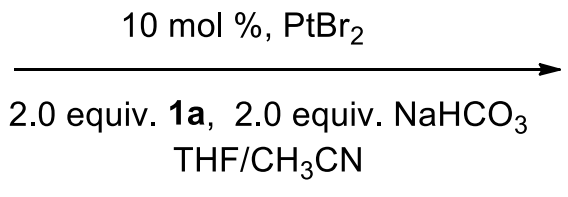

$\mathrm{THF} / \mathrm{CH}_{3} \mathrm{CN}$

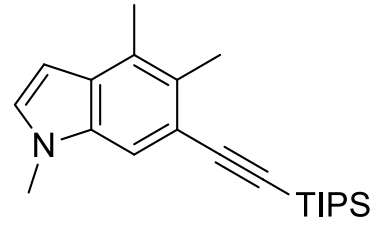

$10 \mathrm{~h}$

Starting from $9 \mathrm{~h}(57 \mathrm{mg}, 0.30 \mathrm{mmol})$, the crude product was purified by column chromatography (Pentane/DCM=30:1) to afford 10h as yellow oil (35 $\mathrm{mg}, 0.10 \mathrm{mmol}, 34 \%$ ).

Rf (Pentane / DCM= 30 / 1): 0.2. ${ }^{1} \mathrm{H}$ NMR (400 MHz, $\left.\mathrm{CDCl}_{3}\right) \delta 7.36$ (s, $1 \mathrm{H}$, benzeneH), 7.04 (d, $1 \mathrm{H}, J=3.1 \mathrm{~Hz}$, pyrroleH), 6.42 (dd, $1 \mathrm{H}, J=3.1,0.8 \mathrm{~Hz}$, pyrroleH), $3.75\left(\mathrm{~s}, 3 \mathrm{H}, \mathrm{NCH}_{3}\right)$, $2.52\left(\mathrm{~s}, 3 \mathrm{H}, \mathrm{CH}_{3}\right), 2.45\left(\mathrm{~s}, 3 \mathrm{H}, \mathrm{CH}_{3}\right), 1.16$ (s, $21 \mathrm{H}$, TIPS). ${ }^{13} \mathrm{C}$ NMR $\left(201 \mathrm{MHz}, \mathrm{CDCl}_{3}\right) \delta$ 134.2, 129.6, 129.5, 128.2, 127.8, 117.2, 111.3, 108.5, 99.5, 91.5, 32.9, 18.8, 17.0, 15.9, 11.5. HRMS IR 2942 (m), 2899 (w), 2140 (w), 1510 (w), 1283 (w), 1085 (w), 997 (w), 912 (w), $885(\mathrm{w}), 849(\mathrm{w}), 714(\mathrm{w})$. (ESI) calcd for $\mathrm{C}_{22} \mathrm{H}_{34} \mathrm{NSi}^{+}[\mathrm{M}+\mathrm{H}]^{+} 340.2455$; found 340.2462.

\section{9-Methyl-2-((triisopropylsilyl)ethynyl)-9H-carbazole (10i)}<smiles>C#CCC(OC)c1cn(C)c2ccccc12</smiles>

$9 \mathbf{i}$

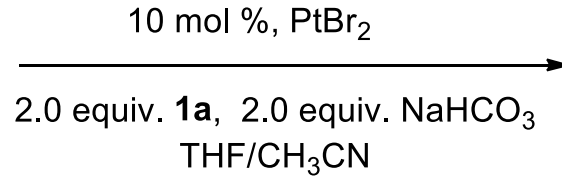

$\mathrm{THF} / \mathrm{CH}_{3} \mathrm{CN}$

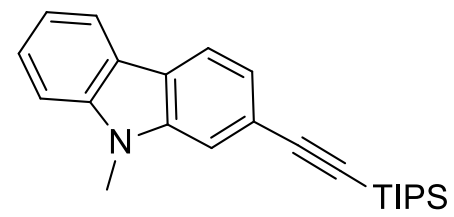

$10 \mathrm{i}$

Starting from 9i $(64 \mathrm{mg}, 0.30 \mathrm{mmol})$, the crude product was purified by column chromatography (Pentane/DCM= 30:1) to afford 10i as yellow oil (63 mg, $0.17 \mathrm{mmol}, 58 \%)$. 
Rf (Pentane / DCM= $30 / 1): 0.4 .{ }^{1} \mathrm{H}$ NMR (400 MHz, $\left.\mathrm{CDCl}_{3}\right) \delta 8.08(\mathrm{~d}, 1 \mathrm{H}, J=7.8 \mathrm{~Hz}$, benzeneH), $8.00(\mathrm{~d}, 1 \mathrm{H}, J=8.0 \mathrm{~Hz}$, benzeneH $), 7.53(\mathrm{~s}, 1 \mathrm{H}$, benzeneH $), 7.50(\mathrm{~m}, 1 \mathrm{H}$, benzeneH), $7.39(\mathrm{~m}, 2 \mathrm{H}$, benzeneH $), 7.25(\mathrm{~m}, 1 \mathrm{H}$, benzene $H), 3.84\left(\mathrm{~s}, 3 \mathrm{H}, \mathrm{NCH}_{3}\right), 1.20(\mathrm{~s}$, $21 \mathrm{H}$, TIPS $).{ }^{13} \mathrm{C}$ NMR $\left(101 \mathrm{MHz}, \mathrm{CDCl}_{3}\right) \delta 141.6,140.5,126.1,123.1,122.8,122.4,120.5$, 120.3, 120.0, 119.1, 112.1, 108.5, 108.4, 89.7, 29.1, 18.8, 11.4. IR 2957 (w), 2891 (w), 2865 (w), $2150(\mathrm{w}), 1601(\mathrm{w}), 1498(\mathrm{w}), 1324(\mathrm{~m}), 1248(\mathrm{w}), 1119(\mathrm{w}), 1018(\mathrm{w}), 940(\mathrm{w}), 909(\mathrm{~m})$, $885(\mathrm{w}), 851(\mathrm{w}), 816(\mathrm{w}), 766(\mathrm{w}), 741$ (s), $725(\mathrm{~m})$. HRMS (ESI) calcd for $\mathrm{C}_{24} \mathrm{H}_{32} \mathrm{NSi}^{+}$ $[\mathrm{M}+\mathrm{H}]^{+}$362.2299; found 362.2307.

\section{6-Methoxy-9-methyl-2-((triisopropylsilyl)ethynyl)-9H-carbazole (10j)}<smiles>C#CCC(OC)c1cn(C)c2ccc(OC)cc12</smiles>

9j

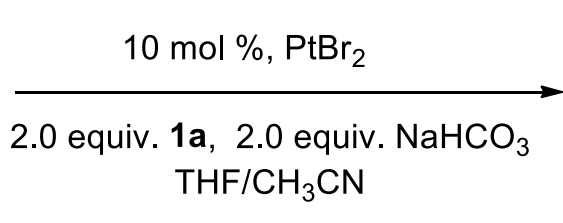

$\mathrm{THF} / \mathrm{CH}_{3} \mathrm{CN}$

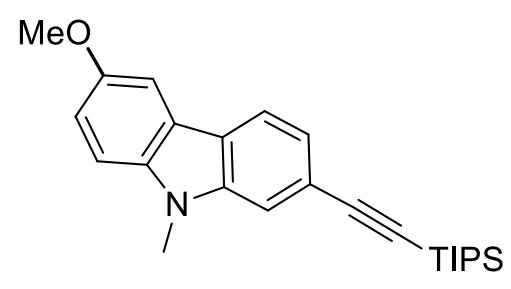

10j

Starting from 9j (73 $\mathrm{mg}, 0.30 \mathrm{mmol})$, the crude product was purified by column chromatography (Pentane/DCM= 30:1) to afford 10j was obtained as yellow oil (75 mg, 0.19 mmol, 64\%).

Rf (Pentane / ether= $30 / 1): 0.5 .{ }^{1} \mathrm{H}$ NMR $\left(400 \mathrm{MHz} \mathrm{CDCl}_{3}\right) \delta 7.95(\mathrm{dd}, 1 \mathrm{H}, J=8.0,0.6 \mathrm{~Hz}$, benzeneH), $7.55(\mathrm{~d}, 1 \mathrm{H}, J=2.4 \mathrm{~Hz}$, benzeneH), $7.49(\mathrm{~d}, 1 \mathrm{H}, J=0.4 \mathrm{~Hz}$, benzeneH), $7.31(\mathrm{~m}$, $2 \mathrm{H}$, benzeneH), $7.13\left(\mathrm{dd}, 1 \mathrm{H}, J=8.8,2.5 \mathrm{~Hz}\right.$, benzeneH), $3.93\left(\mathrm{~s}, 3 \mathrm{H}, \mathrm{NCH}_{3}\right), 3.82(\mathrm{~s}, 3 \mathrm{H}$, $\left.\mathrm{OCH}_{3}\right), 1.18(\mathrm{~m}, 21 \mathrm{H}, \mathrm{TIPS}) .{ }^{13} \mathrm{C} \mathrm{NMR}\left(101 \mathrm{MHz}, \mathrm{CDCl}_{3}\right) \delta 153.8,140.9,136.8,122.7$, 122.6, 120.2, 119.9, 115.5, 112.2, 109.3, 108.5, 103.3, 89.7, 77.2, 56.1, 29.7, 18.8, 11.4. IR 2960 (w), 2186 (w), 1607 (w), 1509 (s), 1463 (w), 1438 (w), 1295 (m), 1250 (s), 1176 (m), 1034 (m), 833 (s), 709 (w). HRMS (ESI) calcd for $\mathrm{C}_{25} \mathrm{H}_{34} \mathrm{NOSi}^{+}[\mathrm{M}+\mathrm{H}]^{+}$392.2404; found 392.2400 . 


\section{Further functionalization:}

\section{5-Ethynyl-1-methyl-1H-indole (39)}

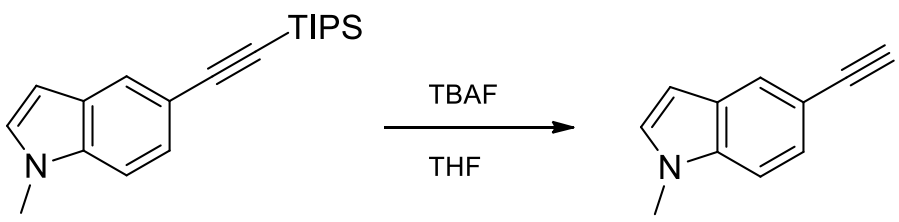

$8 \mathbf{a}$

39

TBAF ( $0.424 \mathrm{~mL}, 0.424 \mathrm{mmol}, 1.2$ equiv) was added slowly into a solution of $\mathbf{8 a}(110 \mathrm{mg}$, $0.353 \mathrm{mmol}, 1.0$ equiv) in THF ( $4 \mathrm{~mL})$ at $0{ }^{\circ} \mathrm{C}$. The reaction was quenched with sat $\mathrm{NH}_{4} \mathrm{Cl}$ solution $(20 \mathrm{~mL})$ after $30 \mathrm{~min}$. The aqueous and organic layers were separated and the aqueous layer was extracted with ether $(3 \times 10 \mathrm{~mL})$. After drying over $\mathrm{MgSO}_{4}$ and concentrating under vacuum, the crude product was purified by column chromatography (Pentane/DCM=10:1) to afford 39 as yellow oil (43 $\mathrm{mg}, 0.28 \mathrm{mmol}, 78 \%$ ).

Rf (Pentane / DCM= $10 / 1): 0.4 .{ }^{1} \mathrm{H}$ NMR (400 MHz, $\left.\mathrm{CDCl}_{3}\right) \delta 7.85$ (s, $1 \mathrm{H}$, benzeneH), 7.38 (dd, $1 \mathrm{H}, J=1.3,8.4 \mathrm{~Hz}$, benzeneH), $7.26(\mathrm{~d}, 1 \mathrm{H}, J=8.4 \mathrm{~Hz}$, benzeneH), 7.07 (d, $1 \mathrm{H}, J=$ $3.1 \mathrm{~Hz}$, pyrroleH), $6.50\left(\mathrm{~d}, 1 \mathrm{H}, J=3.1 \mathrm{~Hz}\right.$, pyrroleH), 3.77 (s, $\left.3 \mathrm{H}, \mathrm{NCH}_{3}\right), 3.04(\mathrm{~s}, 1 \mathrm{H}$, Alkyne H). ${ }^{13} \mathrm{C}$ NMR $\left(101 \mathrm{MHz}, \mathrm{CDCl}_{3}\right) \delta 136.5,129.8,128.1,125.4,113.6,112.5,109.2$, 101.2, 85.4, 74.6, 32.8. IR 1484 (w), 1334 (w), 1245 (w), 886 (w), 805 (w), 728 (w). HRMS (ESI) calcd for $\mathrm{C}_{11} \mathrm{H}_{9} \mathrm{~N}[\mathrm{M}+]$ 156.0808; found 156.0809.

\section{5-Ethynyl-1-methyl-3-((triisopropylsilyl)ethynyl)-1H-indole (11)}

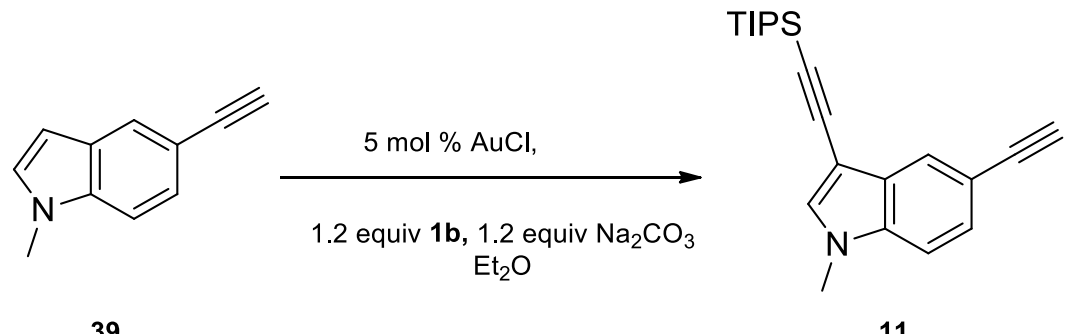

39

11

Gold(I) chloride (3.7 mg, $0.016 \mathrm{mmol}, 0.050$ equiv) was added into a solution of 39 ( $0.050 \mathrm{~g}$, $0.32 \mathrm{mmol}, 1.0$ equiv), $1 \mathbf{b}(0.166 \mathrm{~g}, 0.387 \mathrm{mmol}, 1.2$ equiv), sodium bicarbonate (0.032 $\mathrm{g}$, 0.39 mmol, 1.2 equiv) in $\mathrm{Et}_{2} \mathrm{O}(8 \mathrm{~mL})$. The solvent was removed under vaccum, and the crude product was purified by column chromatography (Pentane/EtOAc $=15: 1)$ directly without any further work-up. 11 was obtained as yellow oil $(68 \mathrm{mg}, 0.20 \mathrm{mmol}, 63 \%, 90 \%$ based on the unconsumed starting material determined from the ${ }^{1} \mathrm{H}$ NMR spectra of crude product)

Rf (Pentane/EtOAc= 15:1): 0.3. ${ }^{1} \mathrm{H}$ NMR $\left(400 \mathrm{MHz}, \mathrm{CDCl}_{3}\right) \delta 7.87(\mathrm{~s}, 1 \mathrm{H}$, benzeneH $), 7.38$ (dd, $1 \mathrm{H}, J=1.5,8.5 \mathrm{~Hz}$, benzeneH), 7.29 (s, $1 \mathrm{H}$, pyrroleH), $7.23(\mathrm{~d}, 1 \mathrm{H}, J=8.5 \mathrm{~Hz}$, benzeneH), 3.75 (s, $\left.3 \mathrm{H}, \mathrm{NCH}_{3}\right), 3.05$ (s, $1 \mathrm{H}$, Alkyne $\left.\mathrm{H}\right), 1.17$ (s, $\left.21 \mathrm{H}, \mathrm{TIPS}\right) .{ }^{13} \mathrm{C} \mathrm{NMR}(101$ $\left.\mathrm{MHz}, \mathrm{CDCl}_{3}\right) \delta 135.8,133.7,129.2,126.6,124.7,113.7,109.6,99.5,98.0,92.2,85.0,75.3$, 33.1, 18.8, 11.4. IR 3311 (w), 3296 (w), 2945 (s), 2893 (w), 2865 (s), 2148 (s), 1479 (m), 
1468 (m), 1374 (m), 1249 (w), 1017 (w), 997 (w), 910 (w), 888 (s), 804 (m), 731 (s). HRMS (ESI) calcd for $\mathrm{C}_{22} \mathrm{H}_{29} \mathrm{NSi}^{+}[\mathrm{M}]^{+} 336.2142$; found 336.2147.

\section{6-Ethynyl-1-methyl-7-(naphthalen-1-yl)-1H-indole (40)}<smiles>Cn1ccc2ccc(C#CC(F)F)c(-c3cccc4ccccc34)c21</smiles>

10d

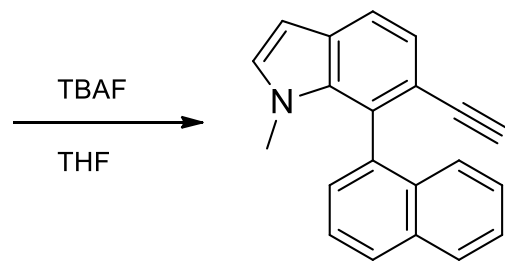

40

TBAF ( $0.18 \mathrm{~mL}, 0.18 \mathrm{mmol}, 1.2$ equiv) was added slowly into a solution of $\mathbf{1 0 d}(65 \mathrm{mg}, 0.15$ mmol, 1.0 equiv) in THF $(3 \mathrm{~mL})$ at $0{ }^{\circ} \mathrm{C}$. The reaction was quenched with a sat $\mathrm{NH}_{4} \mathrm{Cl}$ solution $(20 \mathrm{~mL})$ after $30 \mathrm{~min}$. The aqueous and organic layers were separated and the aqueous layer was extracted with ether $(3 \times 10 \mathrm{~mL})$. After drying over $\mathrm{MgSO}_{4}$ and concentrating under vacuum, the crude product was purified by column chromatography (Pentane/DCM=10:1) to afford $\mathbf{4 0}$ as a yellow oil (35 $\mathrm{mg}, 0.12 \mathrm{mmol}, 84 \%$ ).

Rf (Pentane / DCM= 10 / 1): 0.4. ${ }^{1} \mathrm{H}$ NMR (400 MHz, $\left.\mathrm{CDCl}_{3}\right) \delta$ 7.97-7.93 (m, $2 \mathrm{H}$, benzeneH), $7.66(\mathrm{~d}, 1 \mathrm{H}, J=8.2 \mathrm{~Hz}$, benzeneH $), 7.60-7.54(\mathrm{~m}, 2 \mathrm{H}$, benzeneH $), 7.49(\mathrm{~m}, 1 \mathrm{H}$, benzeneH), 7.42-7.34 (m, $3 \mathrm{H}$, benzeneH), $6.95(\mathrm{~d}, 1 \mathrm{H}, J=3.1 \mathrm{~Hz}$, pyrrole $H), 6.56(\mathrm{~d}, 1 \mathrm{H}, J$ $=3.1 \mathrm{~Hz}$, pyrroleH), $2.85\left(\mathrm{~s}, 3 \mathrm{H}, \mathrm{NCH}_{3}\right), 2.65(\mathrm{~s}, 1 \mathrm{H}$, Alkyne $H) .{ }^{13} \mathrm{C}$ NMR $(101 \mathrm{MHz}$, $\left.\mathrm{CDCl}_{3}\right) \delta 135.7,134.5,133.4,133.1,132.3,129.8,128.4,128.2,128.1,127.6,126.3,125.9$, 124.9, 124.0, 120.3, 116.1, 101.1, 83.9, 78.6, 35.5 (one carbon signal not resolved). IR 3305 (w), $1325(\mathrm{w}), 1324(\mathrm{w}), 1315(\mathrm{w}), 907$ (s), $815(\mathrm{w}), 806(\mathrm{w}), 779(\mathrm{w}), 728(\mathrm{~s})$. HRMS (ESI) calcd for $\mathrm{C}_{21} \mathrm{H}_{15} \mathrm{~N}^{+}[\mathrm{M}]^{+} 282.1277$; found 282.1284.

\section{1-Methyl-1H-phenanthro[3,4-g]indole (12)}

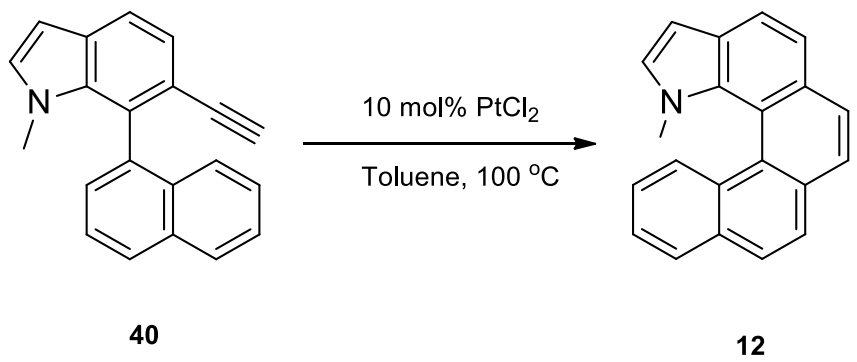

$\mathrm{PtCl}_{2}$ (3.3 mg, $0.012 \mathrm{mmol}, 0.1$ equiv) was added into a solution of $\mathbf{4 0}$ (35 $\mathrm{mg}, 0.12 \mathrm{mmol}$, 1.0 equiv) in toluene $(4.8 \mathrm{~mL})$. The mixture was kept at $100{ }^{\circ} \mathrm{C}$ overnight. The crude product was purified by column chromatography (Pentane/DCM=10:1) directly to afford 12 as a yellow oil (26 mg, $0.092 \mathrm{mmol}, 74 \%)$.

Rf (Pentane / DCM= 10 / 1): 0.4. ${ }^{1} \mathrm{H}$ NMR (400 MHz, $\left.\mathrm{CDCl}_{3}\right) \delta$ 8.31-8.29 (m, $1 \mathrm{H}$, benzeneH), 7.99-7.97 (m, $2 \mathrm{H}$, benzeneH), 7.92-7.90 (m, $3 \mathrm{H}$, benzeneH), $7.76(\mathrm{~d}, 1 \mathrm{H}, J=8.3$ $\mathrm{Hz}$, benzeneH), $7.68(\mathrm{~d}, 1 \mathrm{H}, J=8.3 \mathrm{~Hz}$, benzeneH $), 7.52(\mathrm{~m}, 1 \mathrm{H}$, benzeneH $), 7.40(\mathrm{~m}, 1 \mathrm{H}$, benzeneH), 7.07 (d, $1 \mathrm{H}, J=3.1 \mathrm{~Hz}$, pyrroleH), $6.81(\mathrm{~d}, 1 \mathrm{H}, J=3.1 \mathrm{~Hz}$, pyrroleH), 2.96 (s, 3 $\left.\mathrm{H}, \mathrm{NCH}_{3}\right) .{ }^{13} \mathrm{C}$ NMR $\left(101 \mathrm{MHz}, \mathrm{CDCl}_{3}\right) \delta 134.5,131.6,131.4,131.0,131.0,130.3,128.3$, $128.0,127.9,126.7,126.2,125.9,125.6,125.2,124.8,124.2,121.2,120.6,116.6,103.3,37.2$. IR $3052(\mathrm{w}), 1344(\mathrm{w}), 1265(\mathrm{~m}), 840(\mathrm{w}), 819(\mathrm{w}), 790(\mathrm{w}), 773(\mathrm{w}), 734(\mathrm{~s}), 705$ (m).HRMS (ESI) calcd for $\mathrm{C}_{21} \mathrm{H}_{15} \mathrm{~N}^{+}[\mathrm{M}]^{+}$282.1277; found 282.1280. 
6. Spectra for new comprounds 
solvent: $\langle\mathrm{CDCl} 3>$

Frequency. $400.13 \mathrm{MHz}$

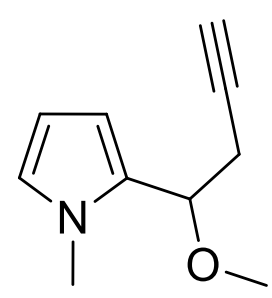

$6 a$

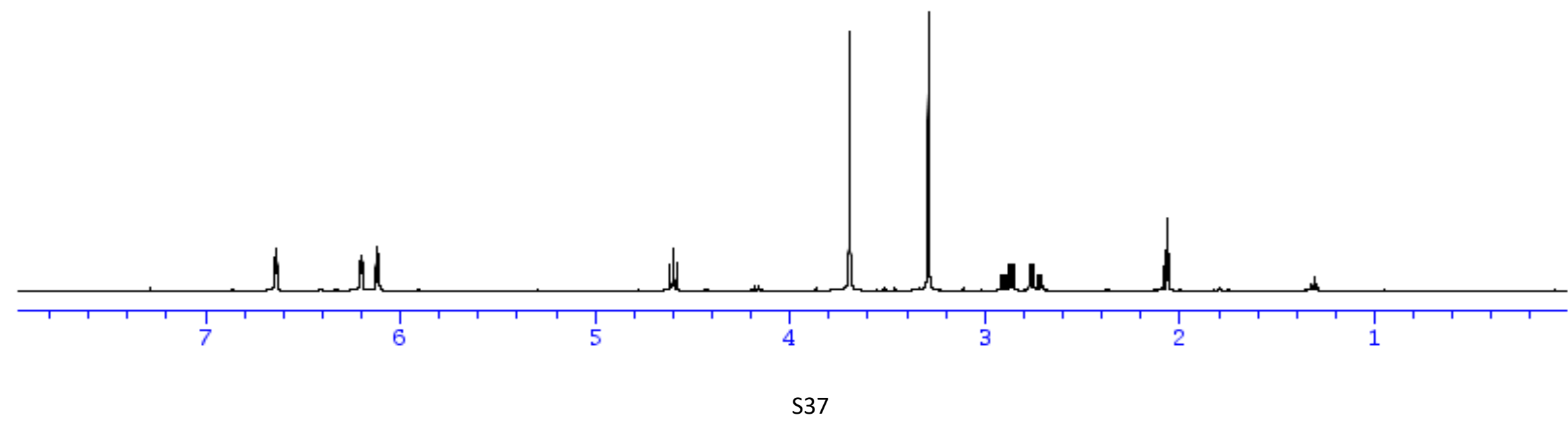


solvent: $\langle\mathrm{CDCl} 3\rangle$

Frequency. 100.612769M Hz

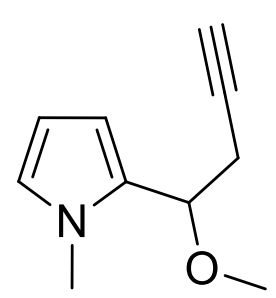

$6 a$

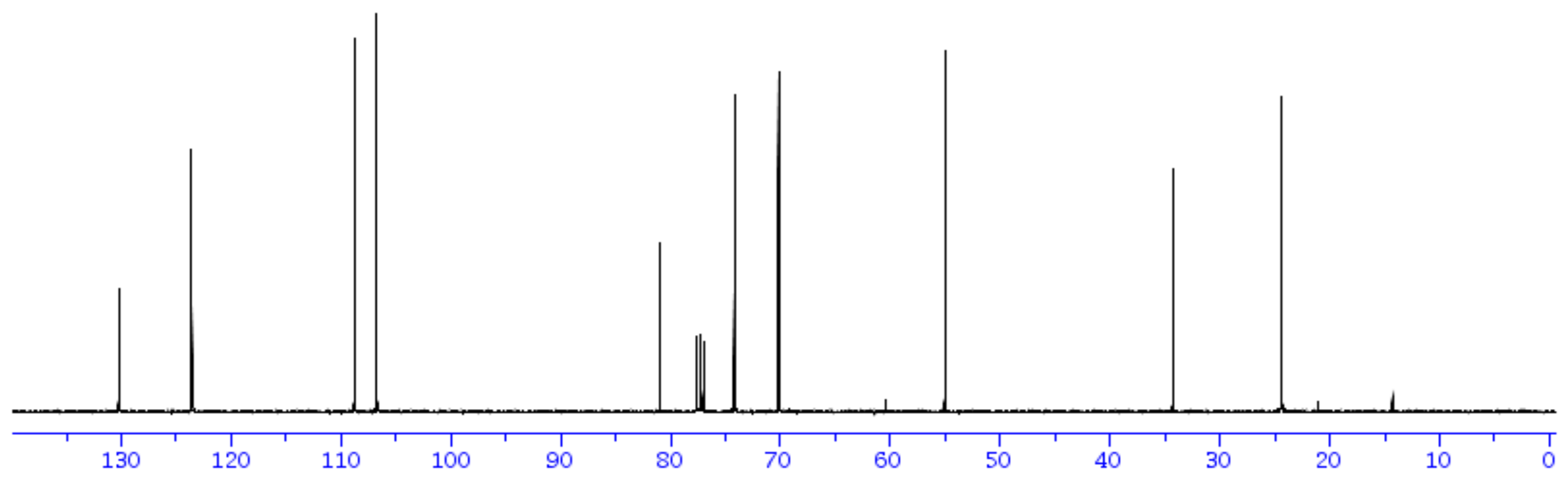




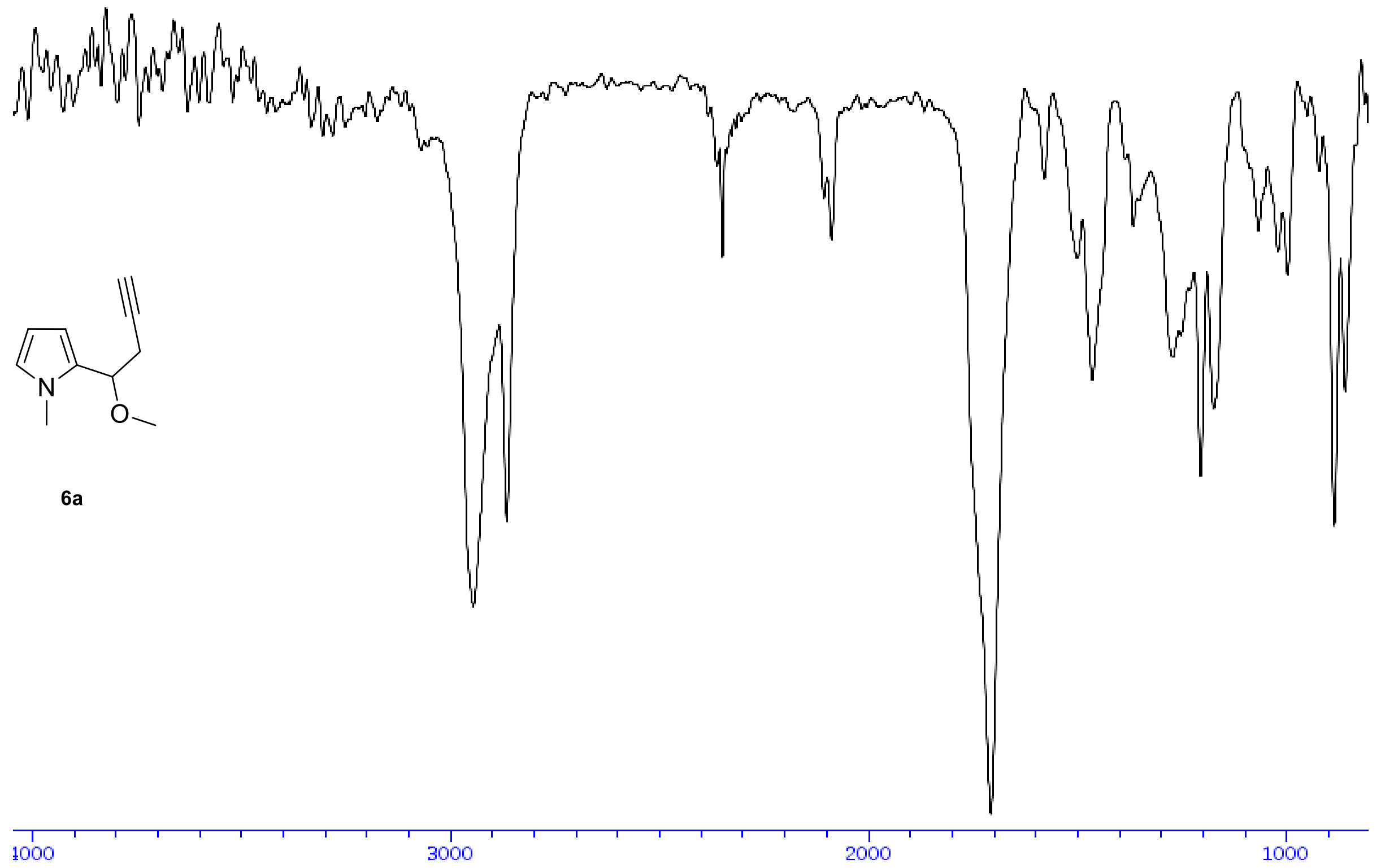

S39 


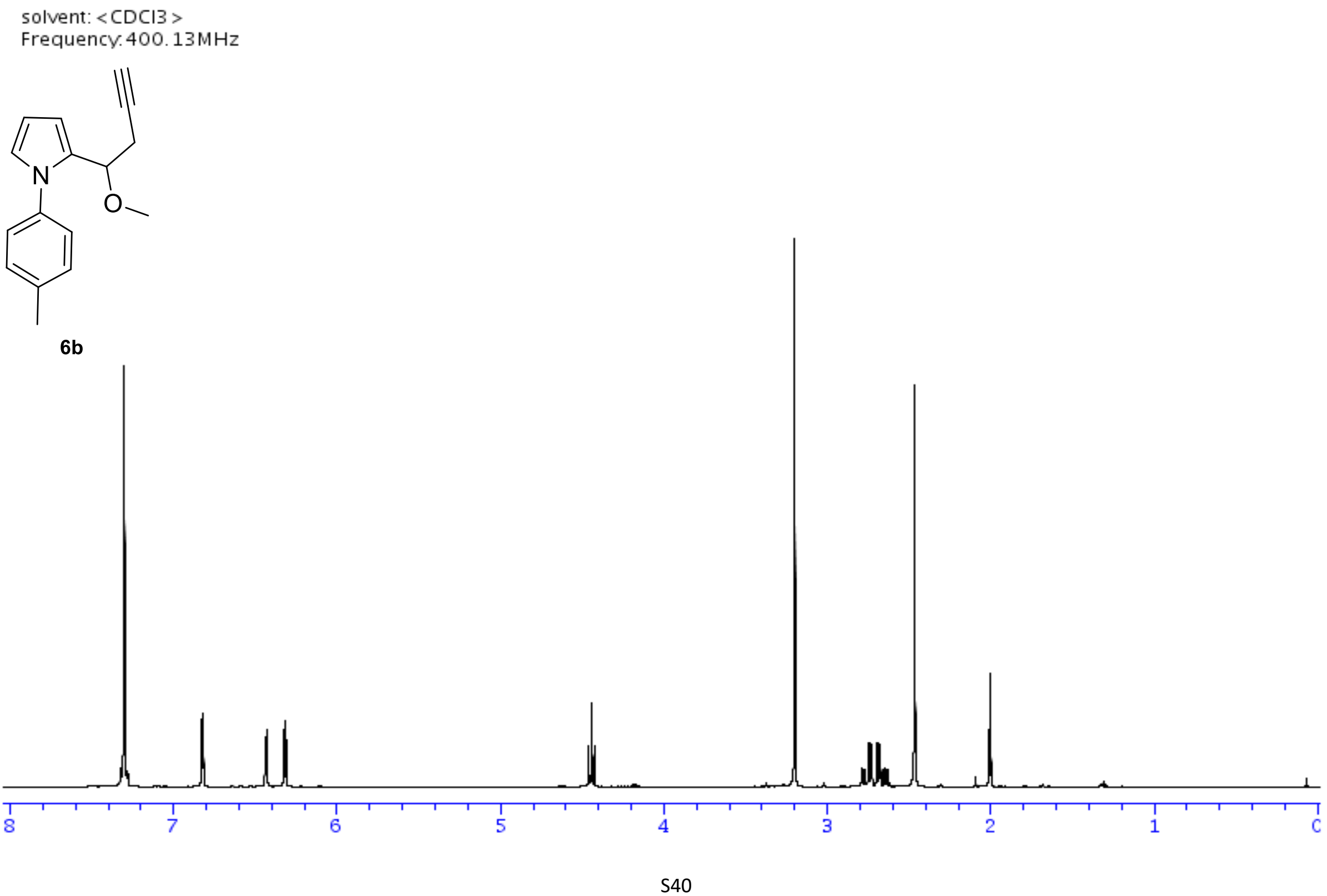


solvent: $\langle\mathrm{CDCl} 3\rangle$

Frequency. $100.612769 \mathrm{MHz}$
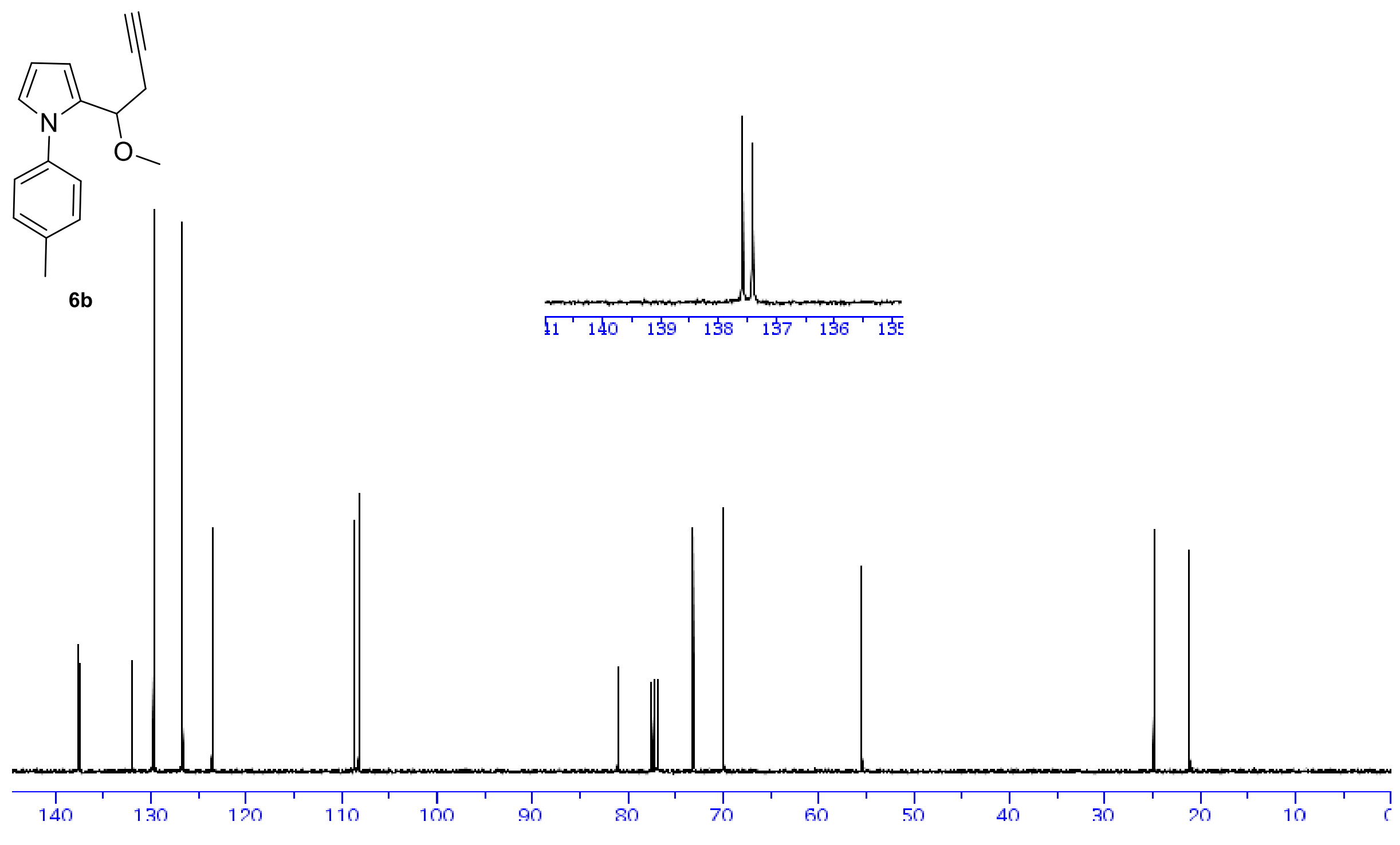


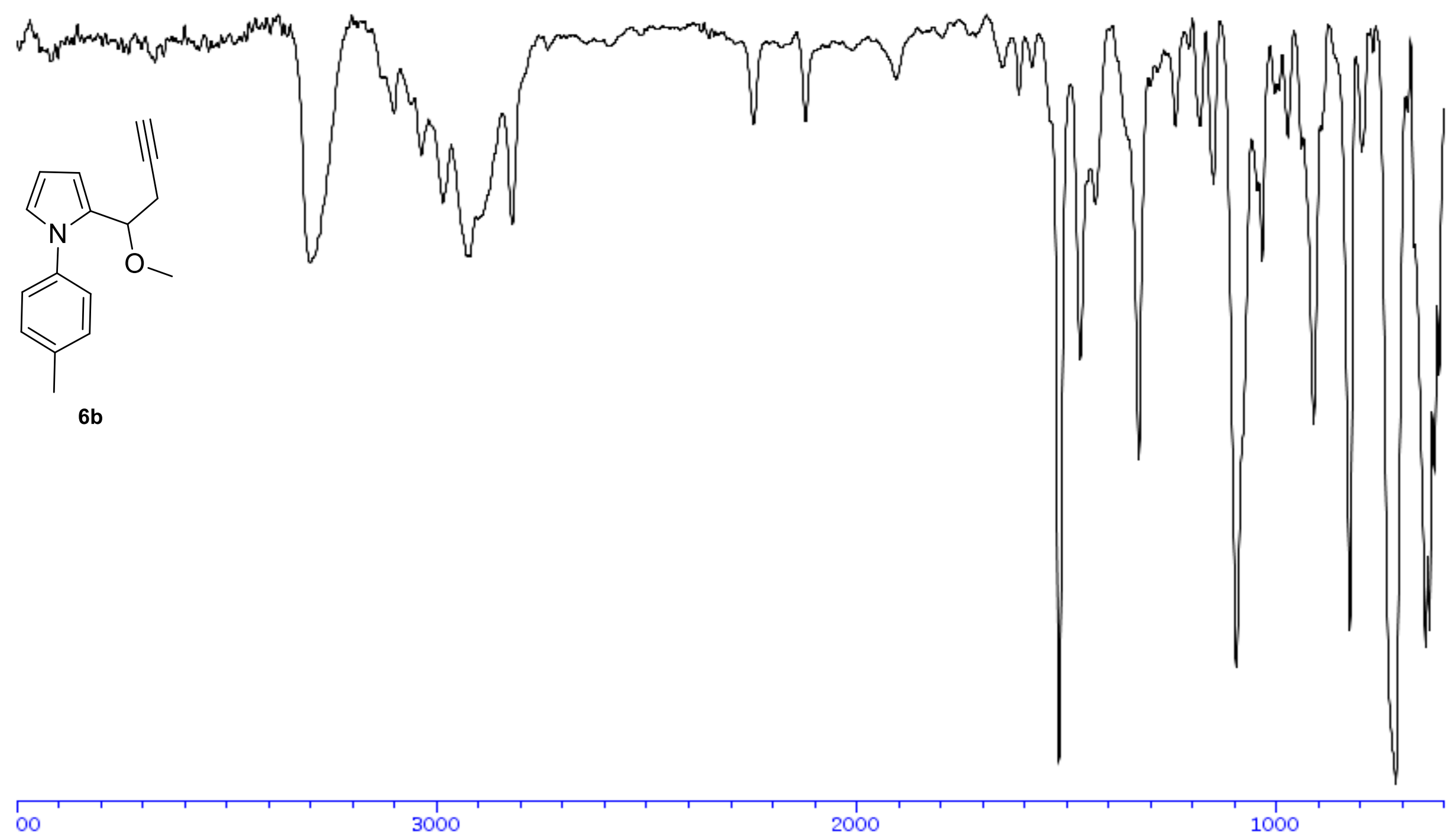


solvent: $\langle\mathrm{CDCl} 3\rangle$

Frequency. 400.13 MHz

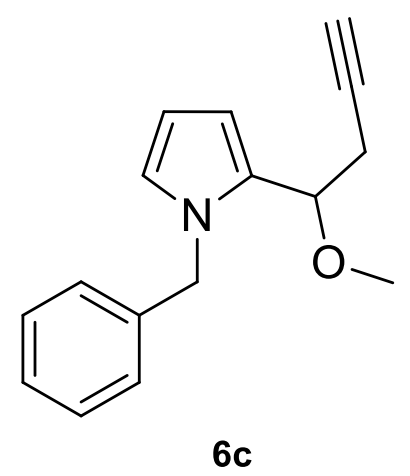

$6 c$

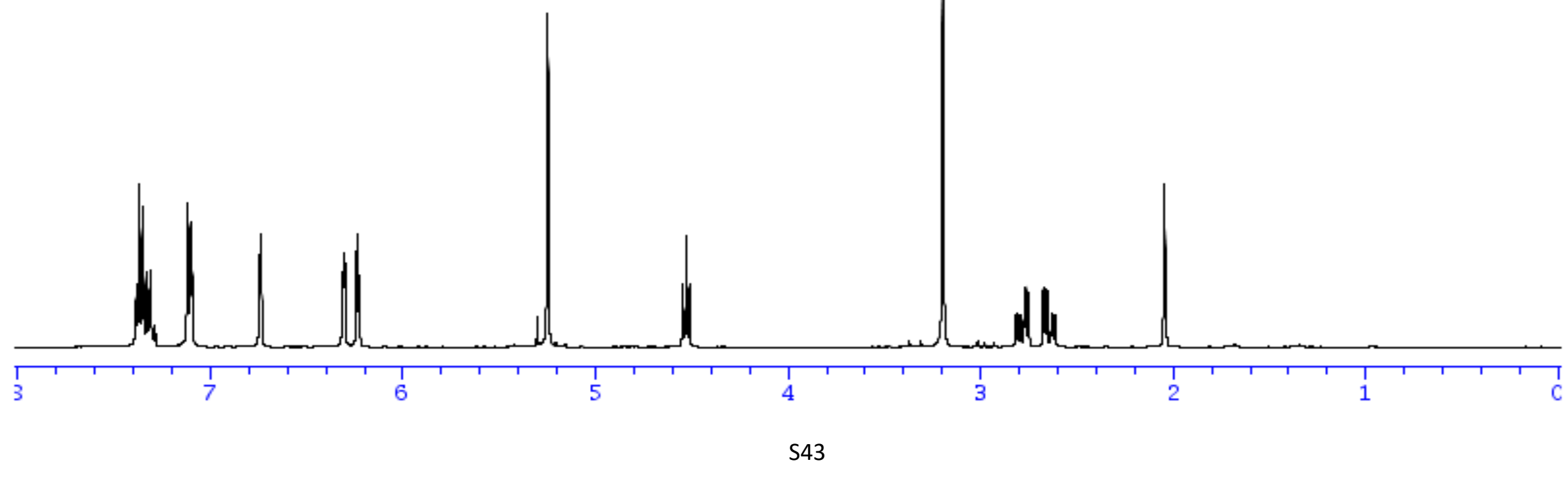


solvent: $<\mathrm{CDCI}$ >

Frequency. 100.612769M Hz
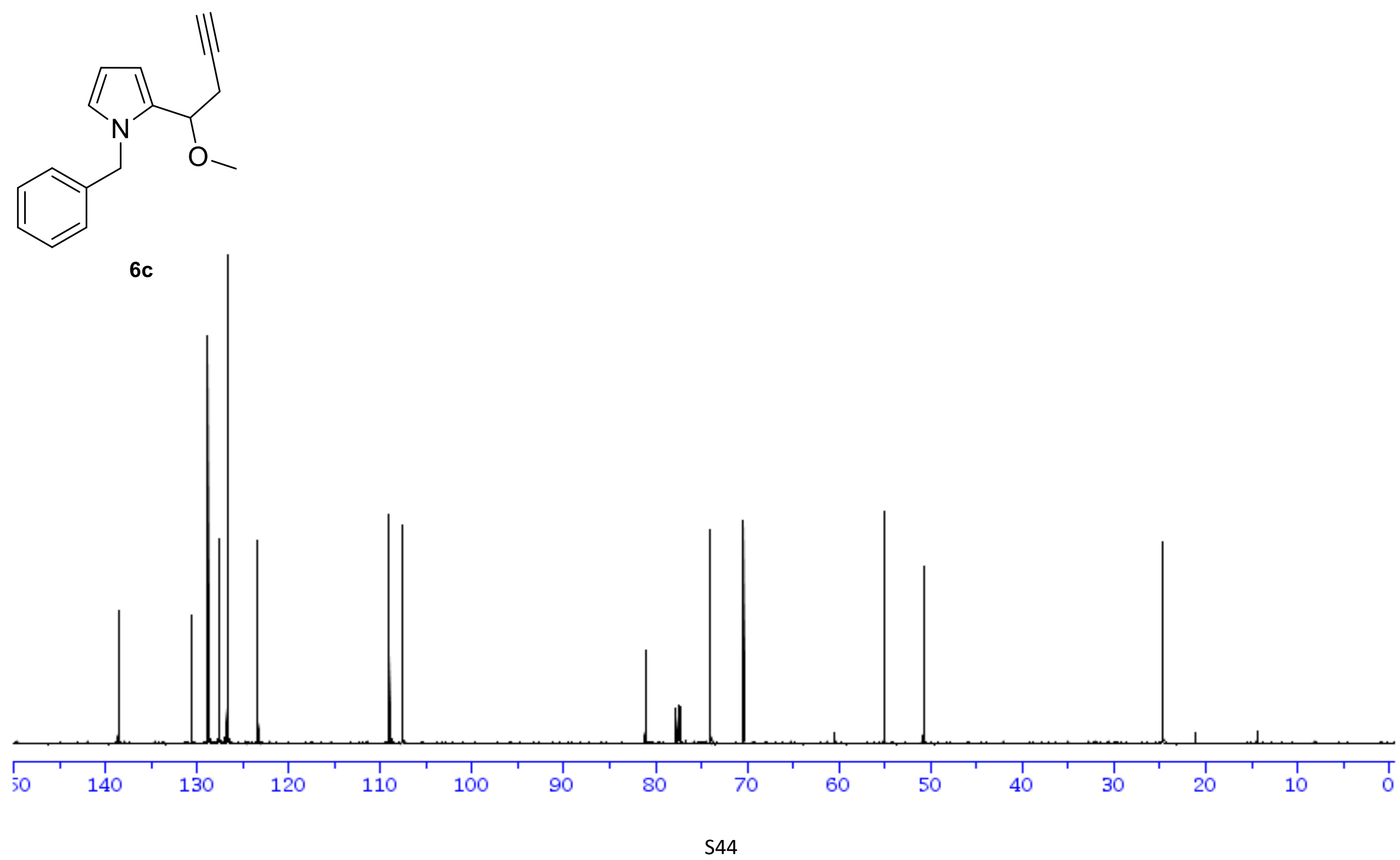


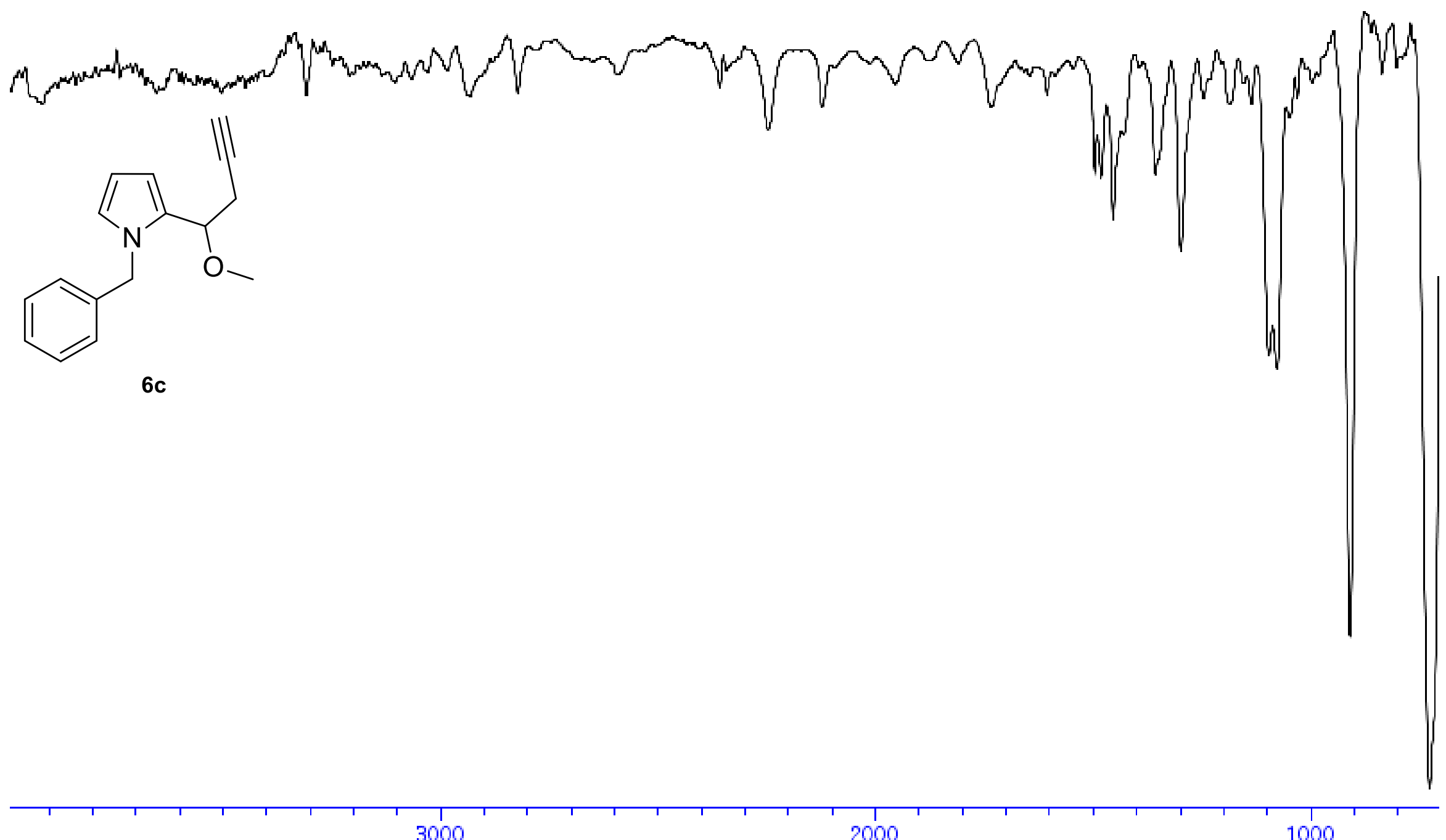

S45 
solvent: $\langle\mathrm{CDCI} 3\rangle$

Frequency. 400.13 MHz

-

$6 d$

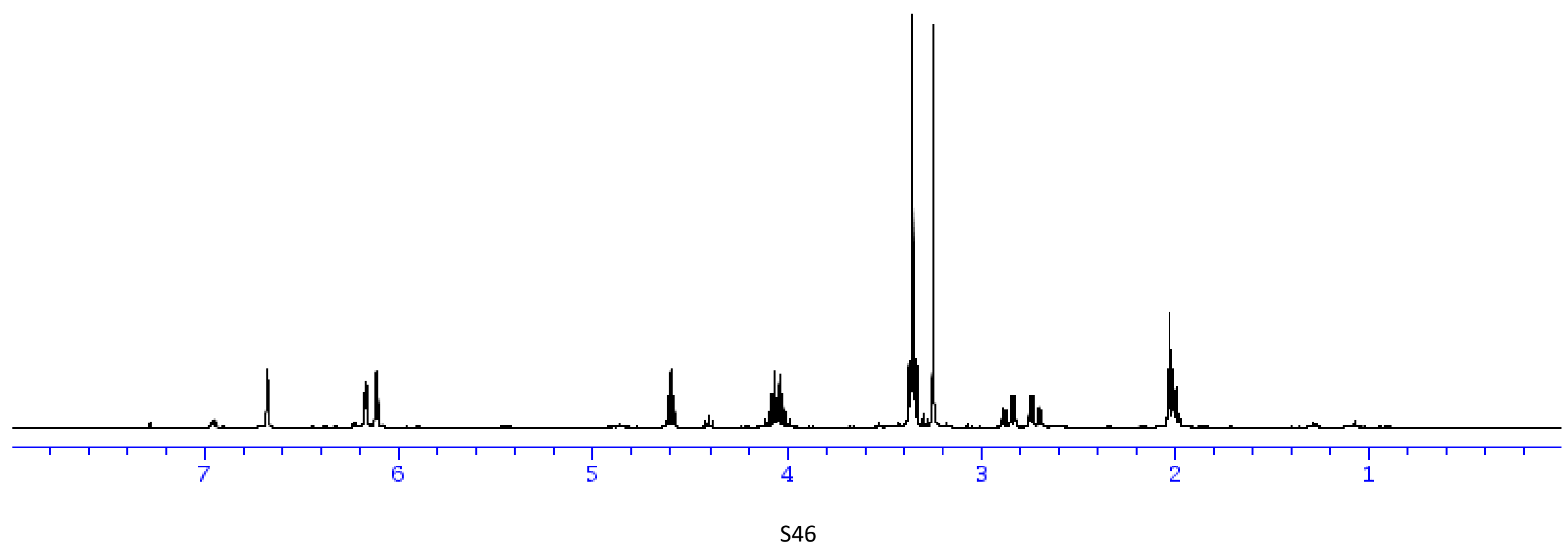


solvent: $\langle\mathrm{CDCI} 3>$

Frequency. 100.612769M Hz

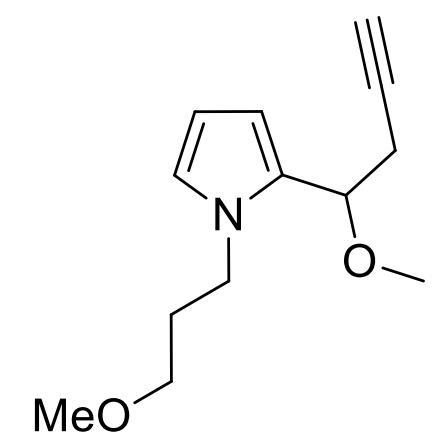

$6 d$

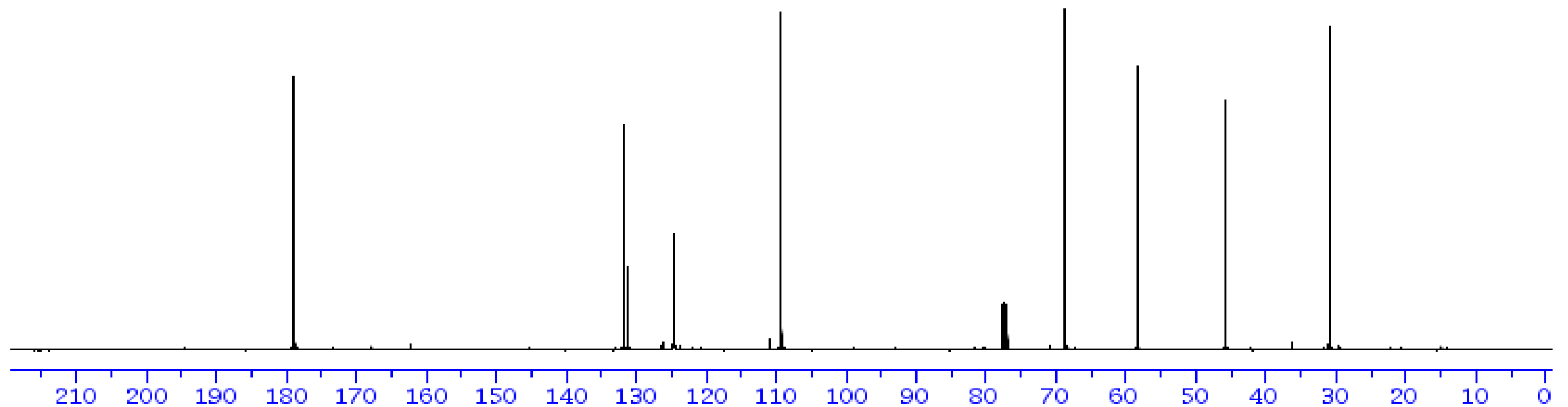




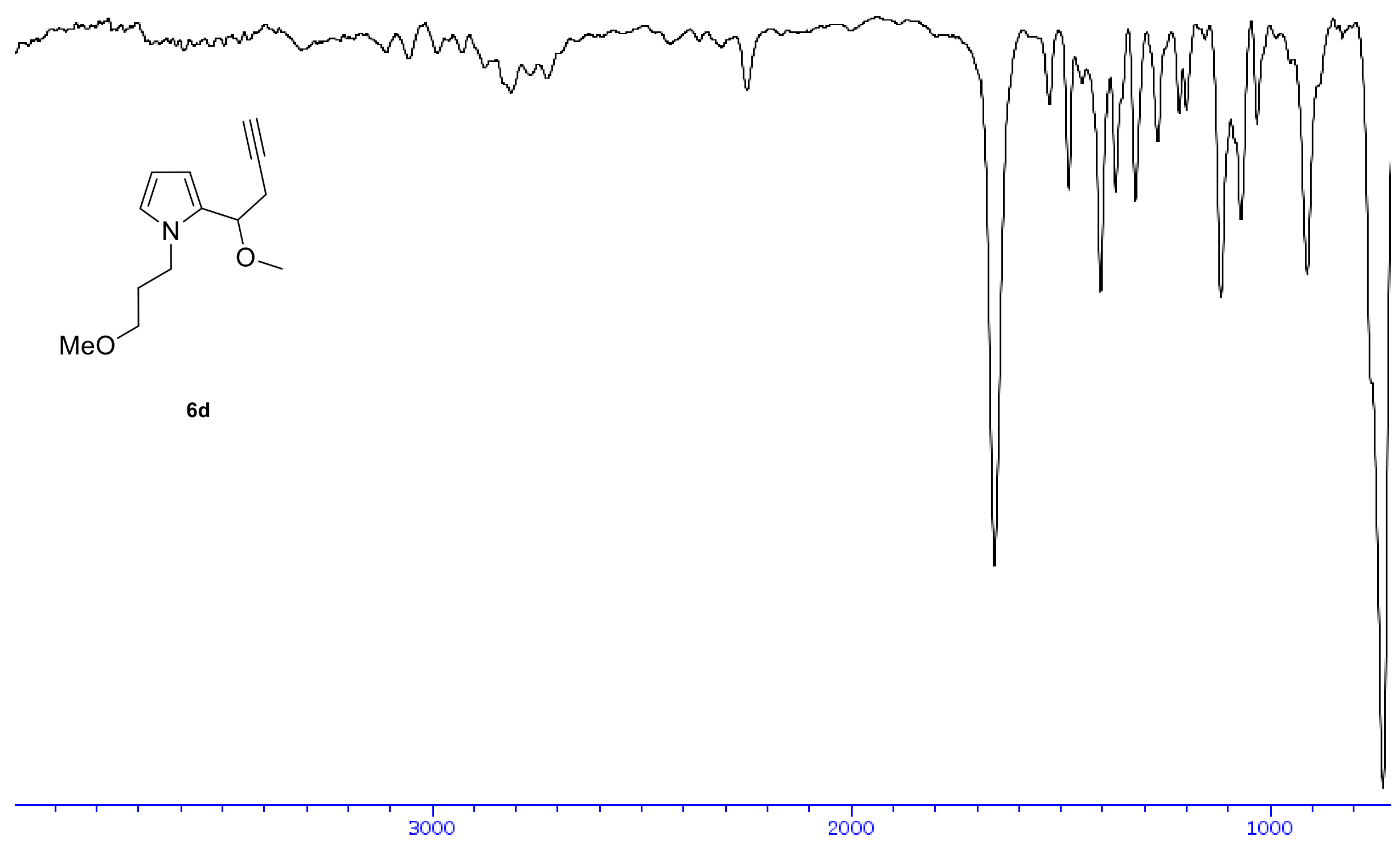


solvent: $<C D C I 3>$

Frequency. $400.13 \mathrm{MHz}$

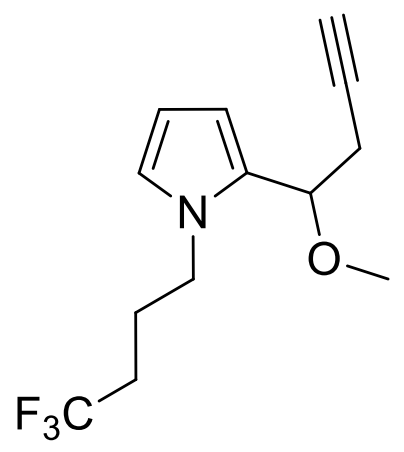

$6 e$

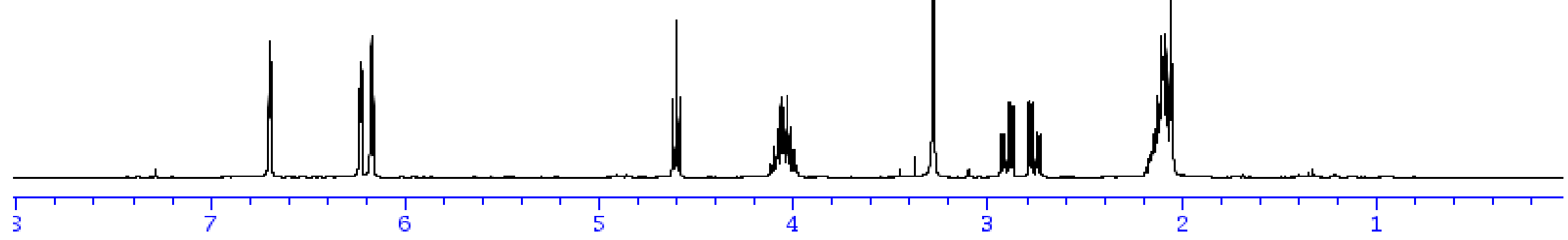

S49 
solvent: $\langle\mathrm{CDCI} 3>$

Frequency. 100.612769M Hz
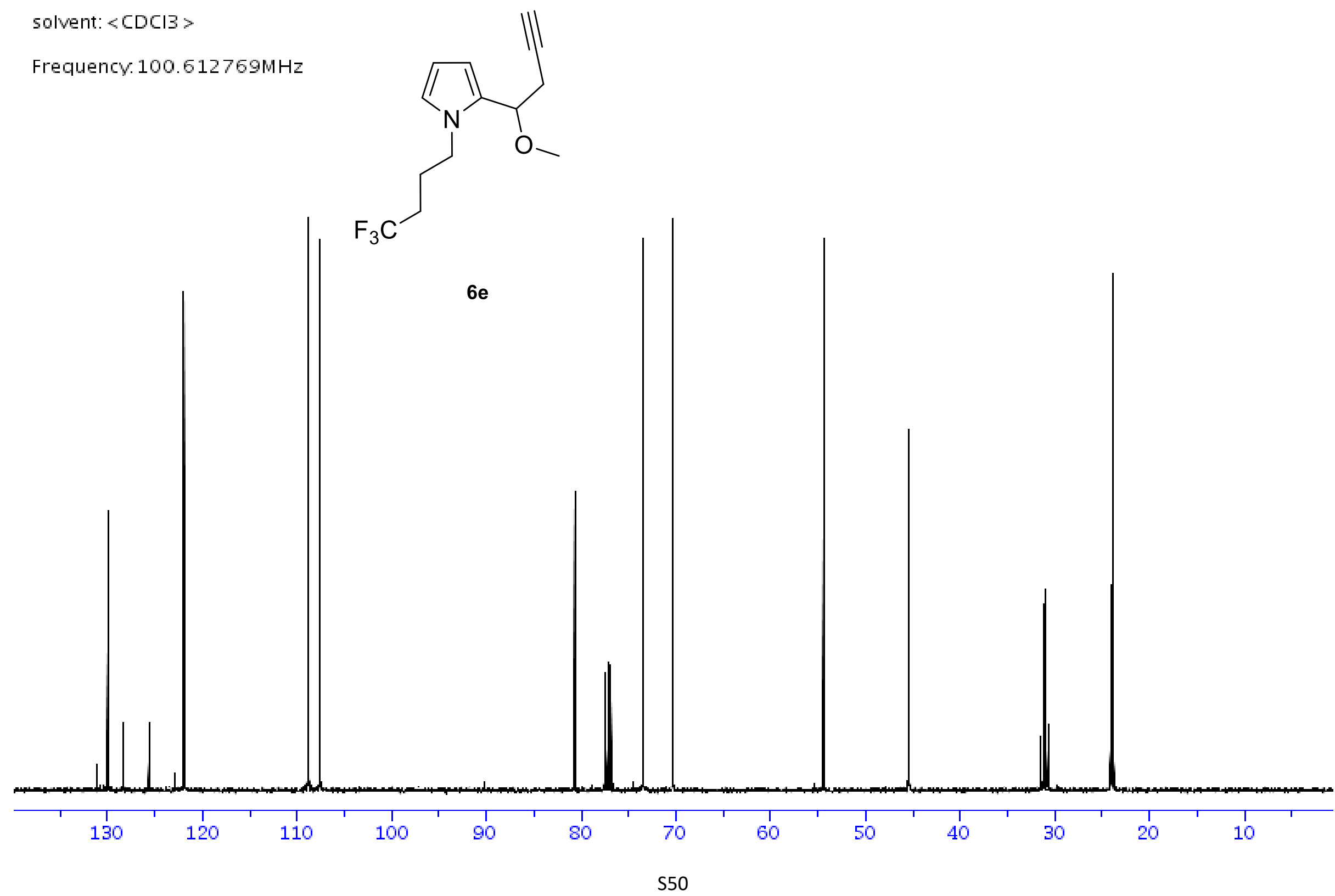


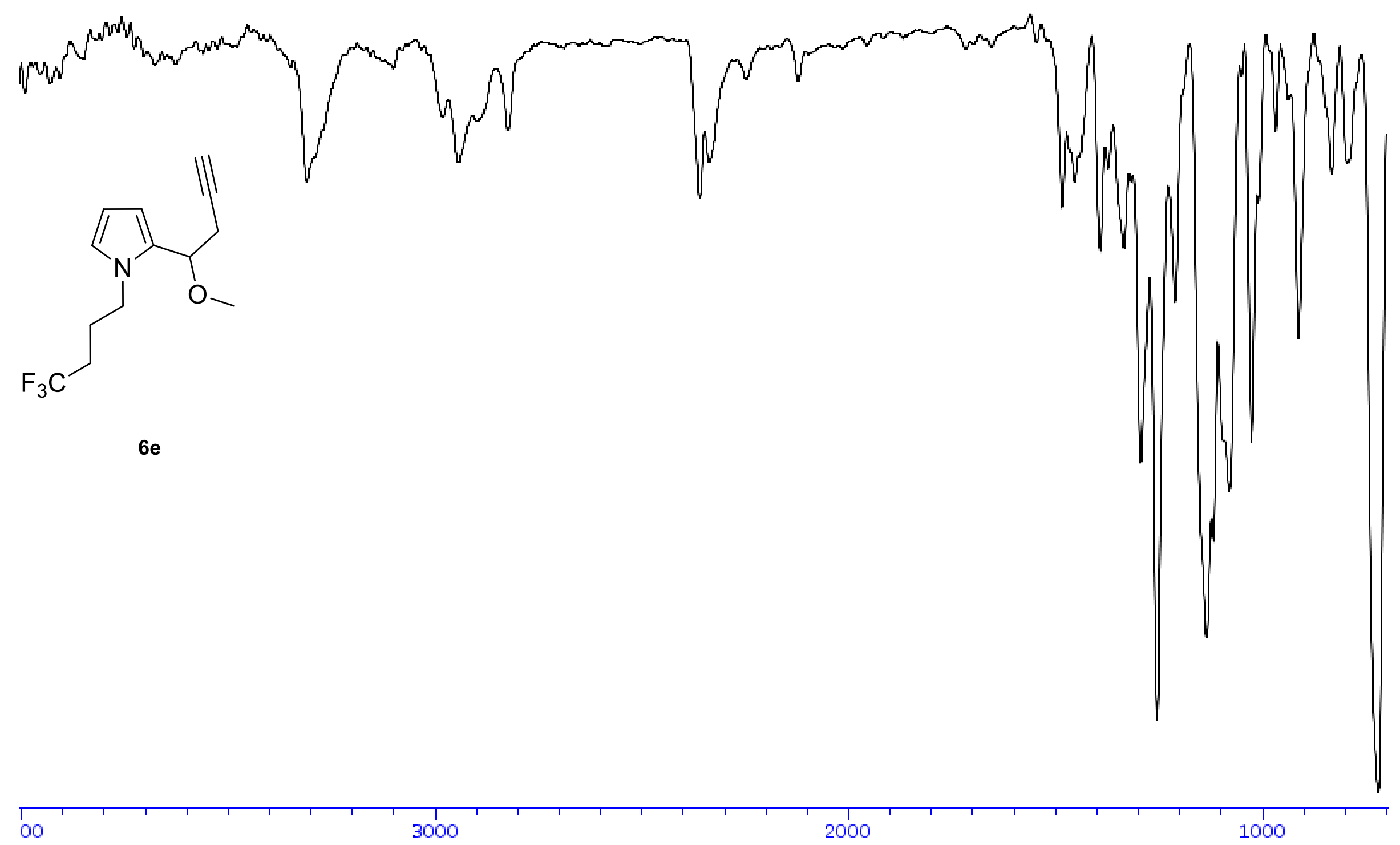




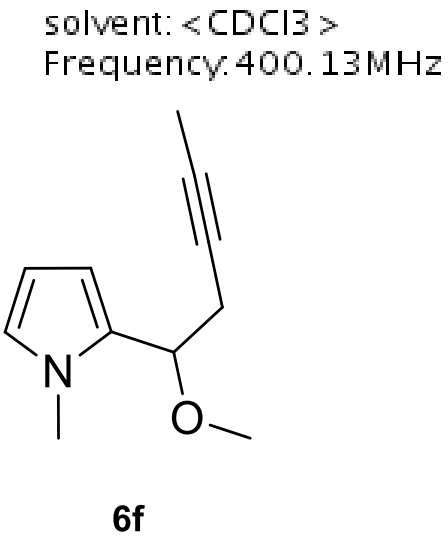

$6 f$

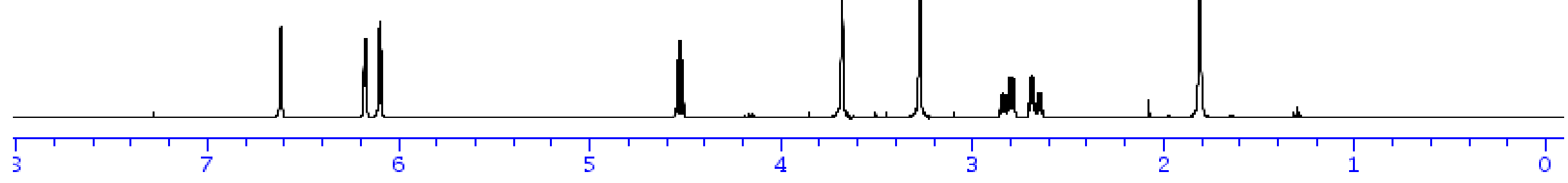

S52 
solvent: $\langle\mathrm{CDCl} 3\rangle$

Frequency. 100.612769M Hz

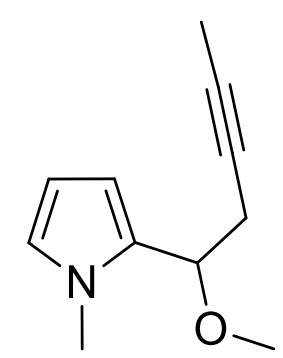

$6 f$
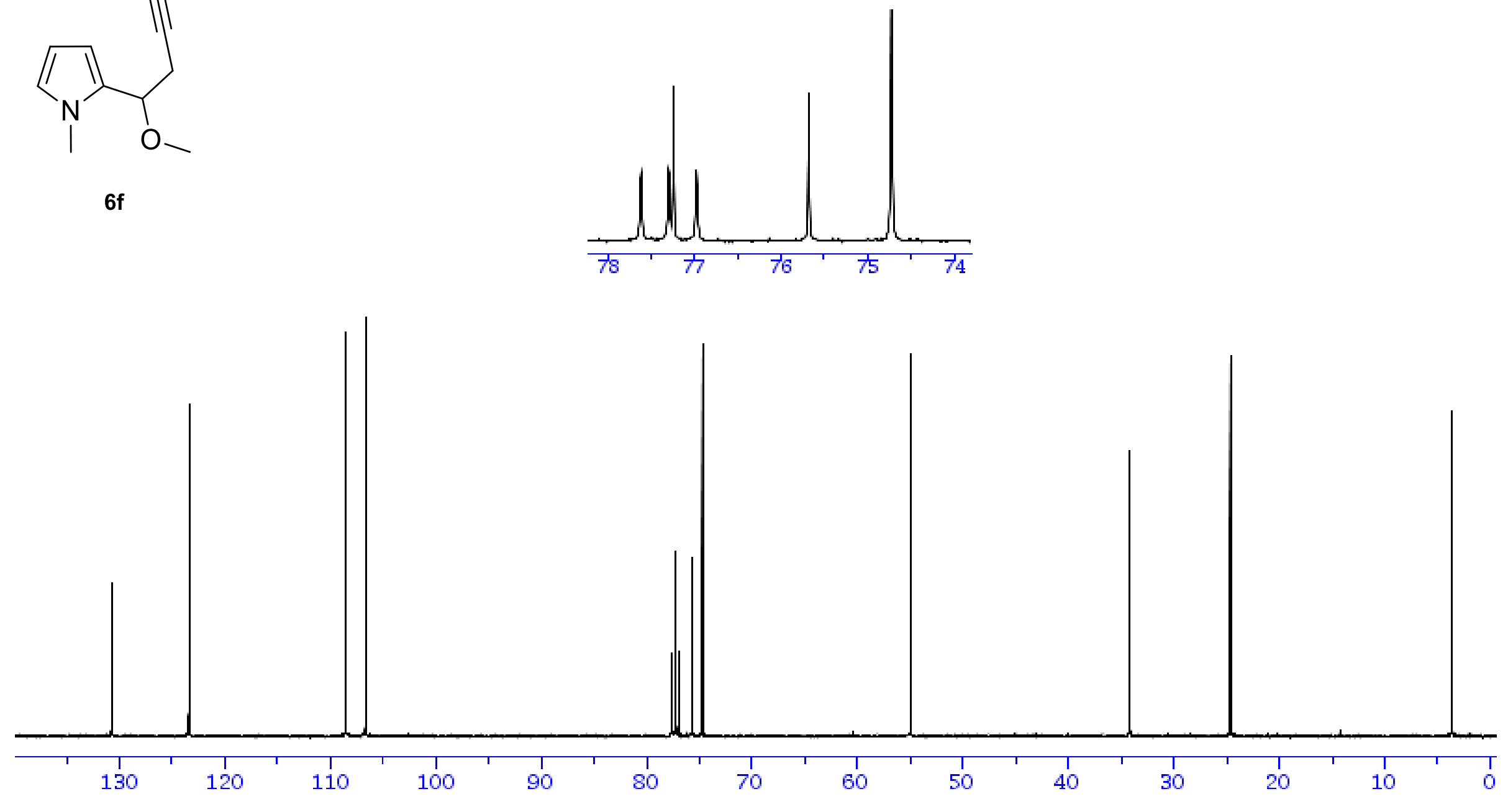


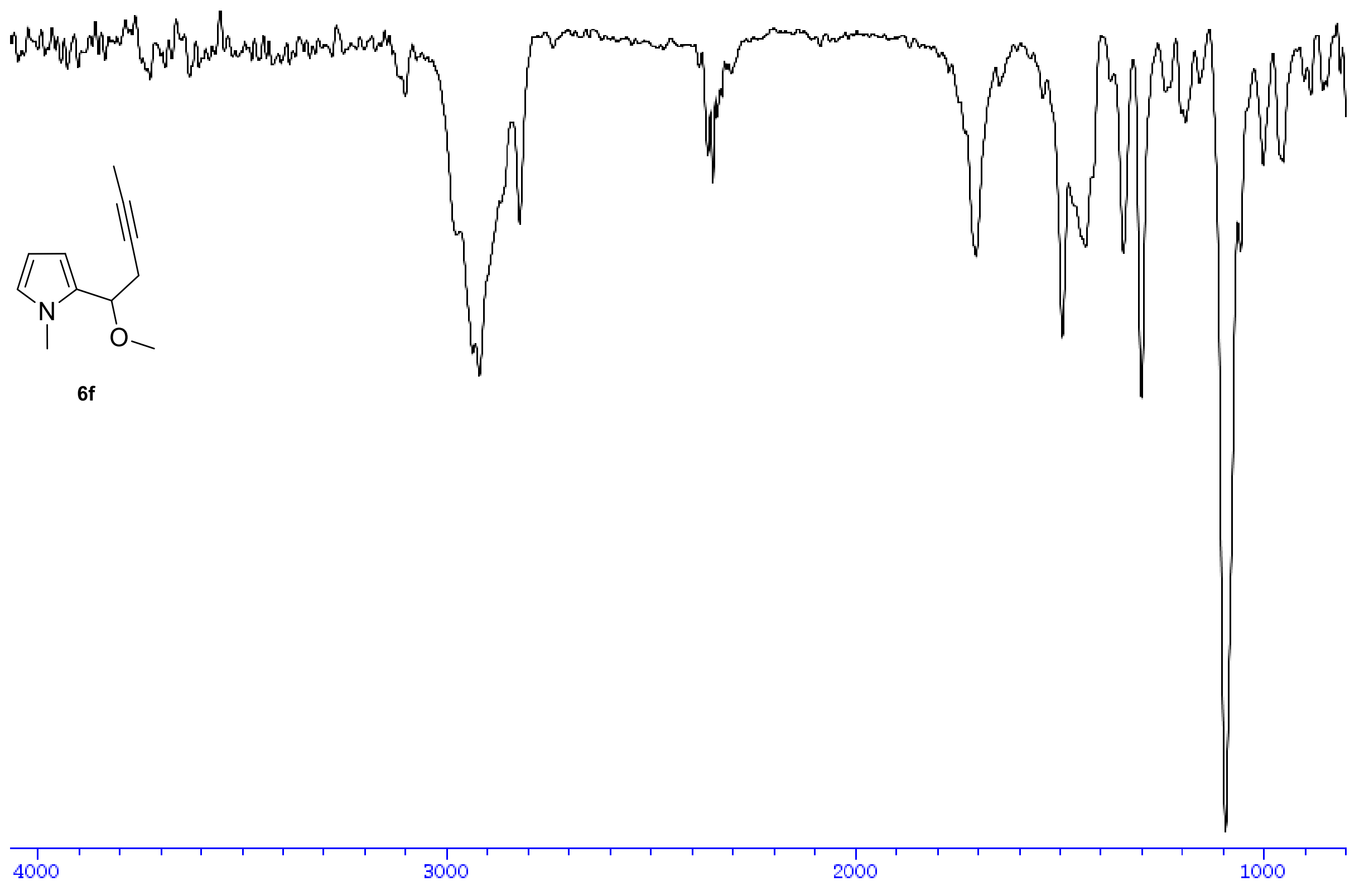

S54 
solvent: $\langle\mathrm{CDCI} 3\rangle$

Frequency. $400.13 \mathrm{MHz}$

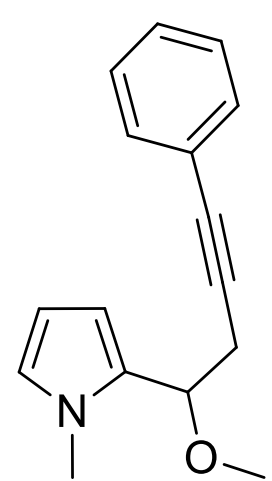

$6 g$

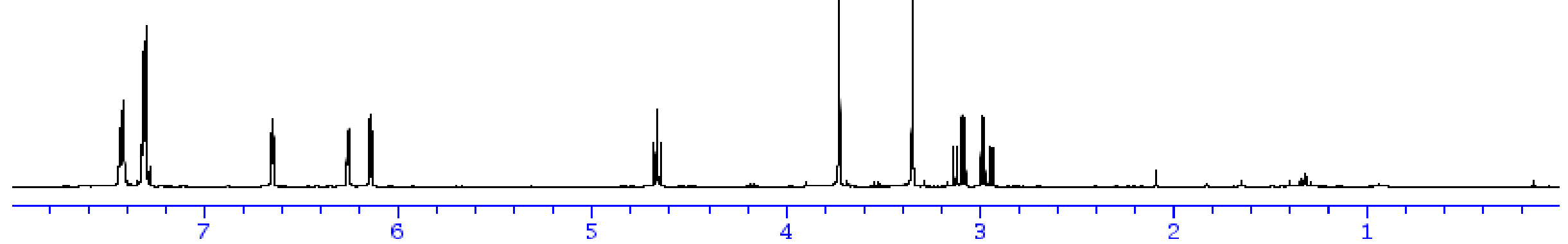


solvent: $<\mathrm{CDCI} 3>$

Frequency. $100.612769 \mathrm{MHz}$

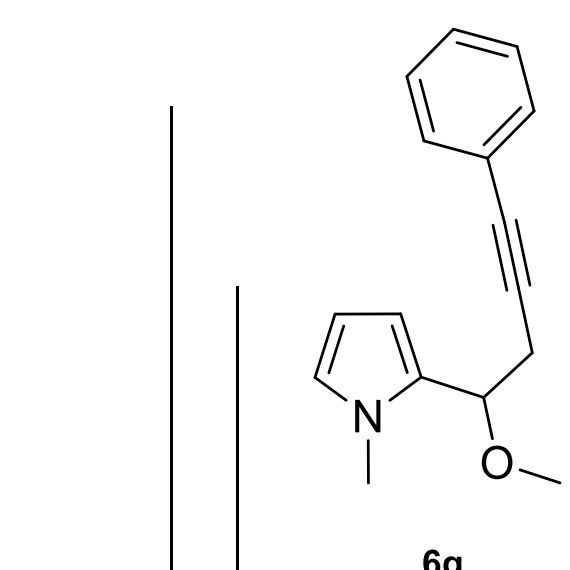

$6 g$
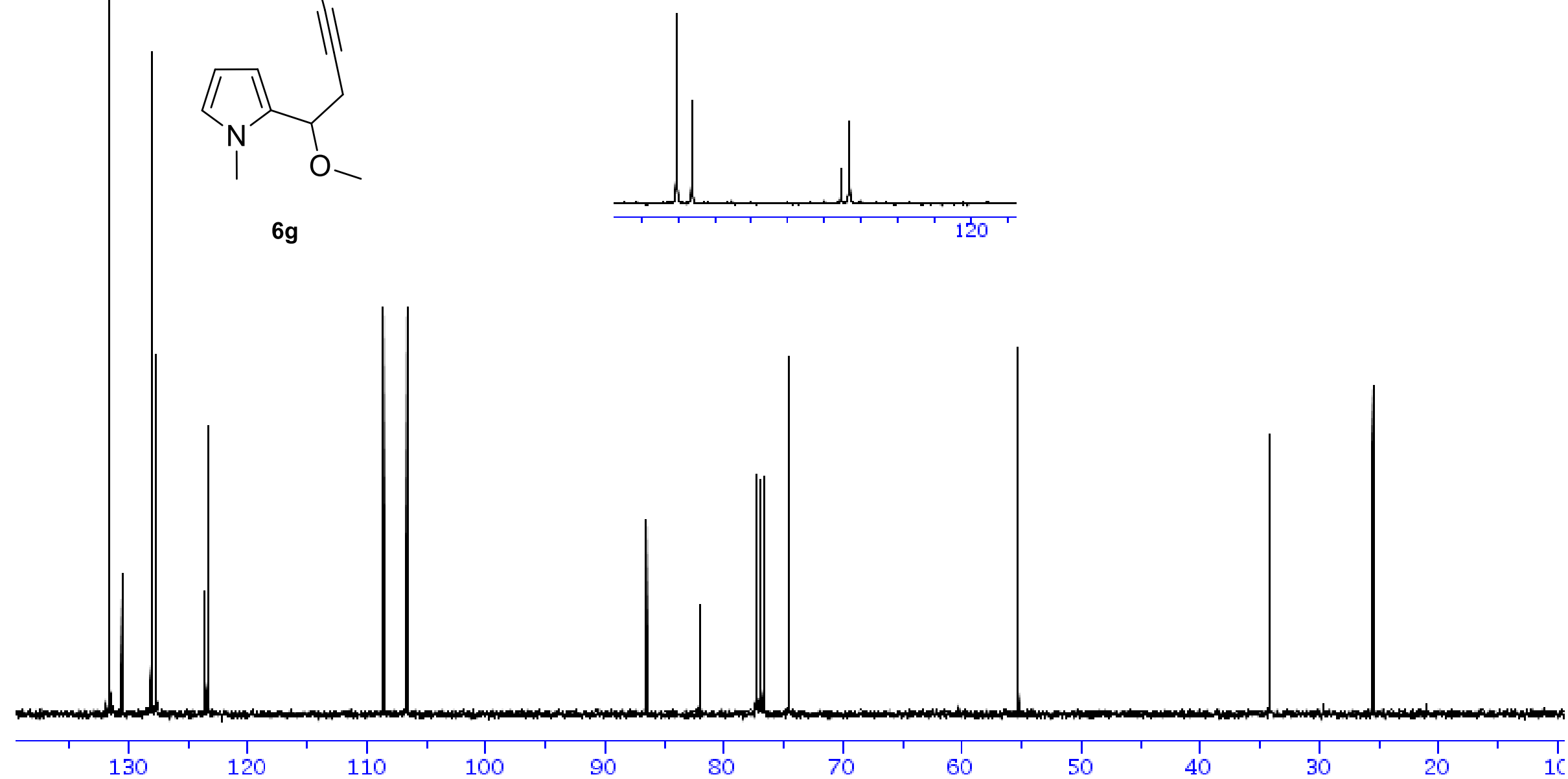


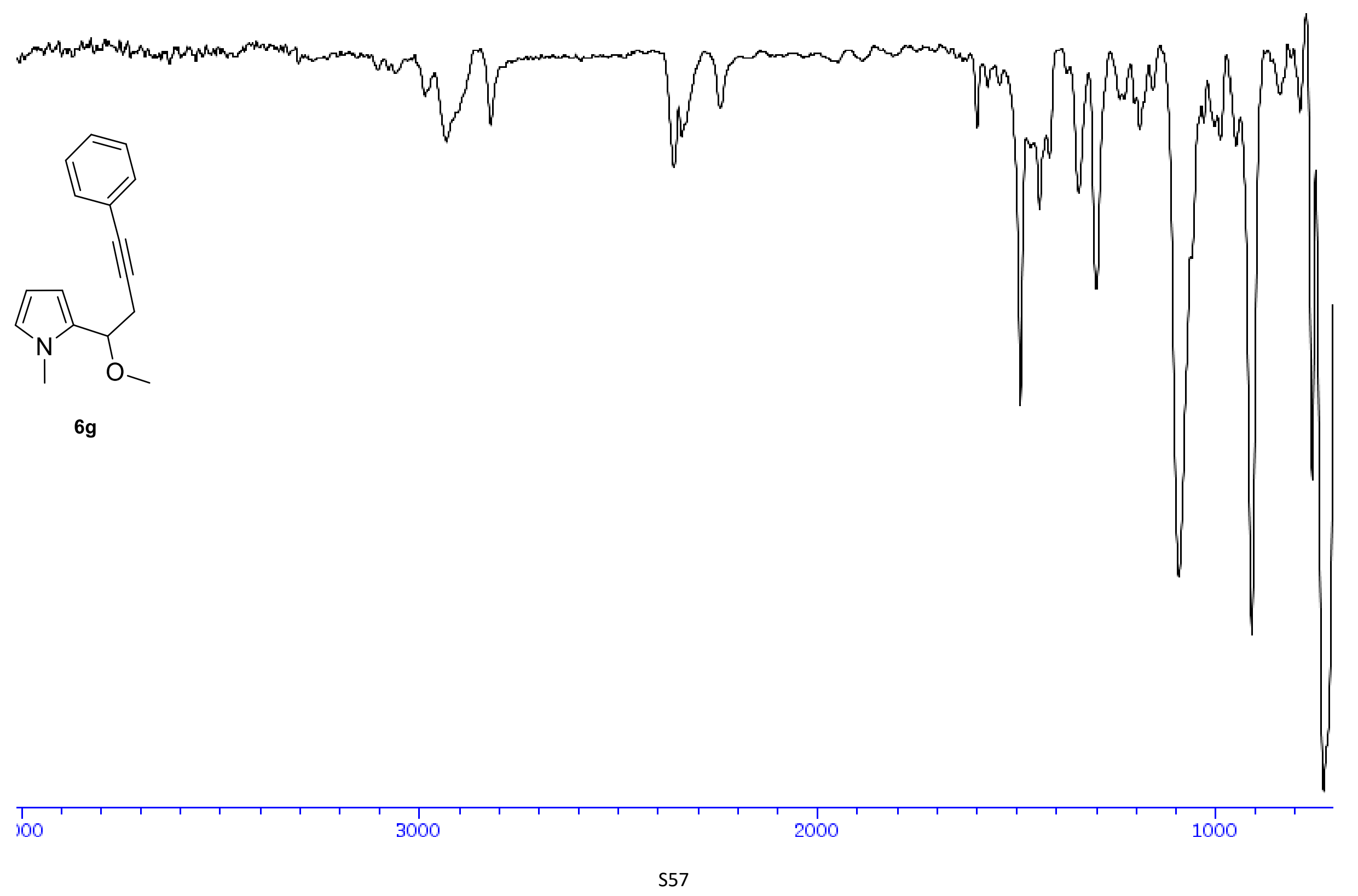


solvent: $<\mathrm{CDCI} 3>$

Frequency. $400.13 \mathrm{MHz}$
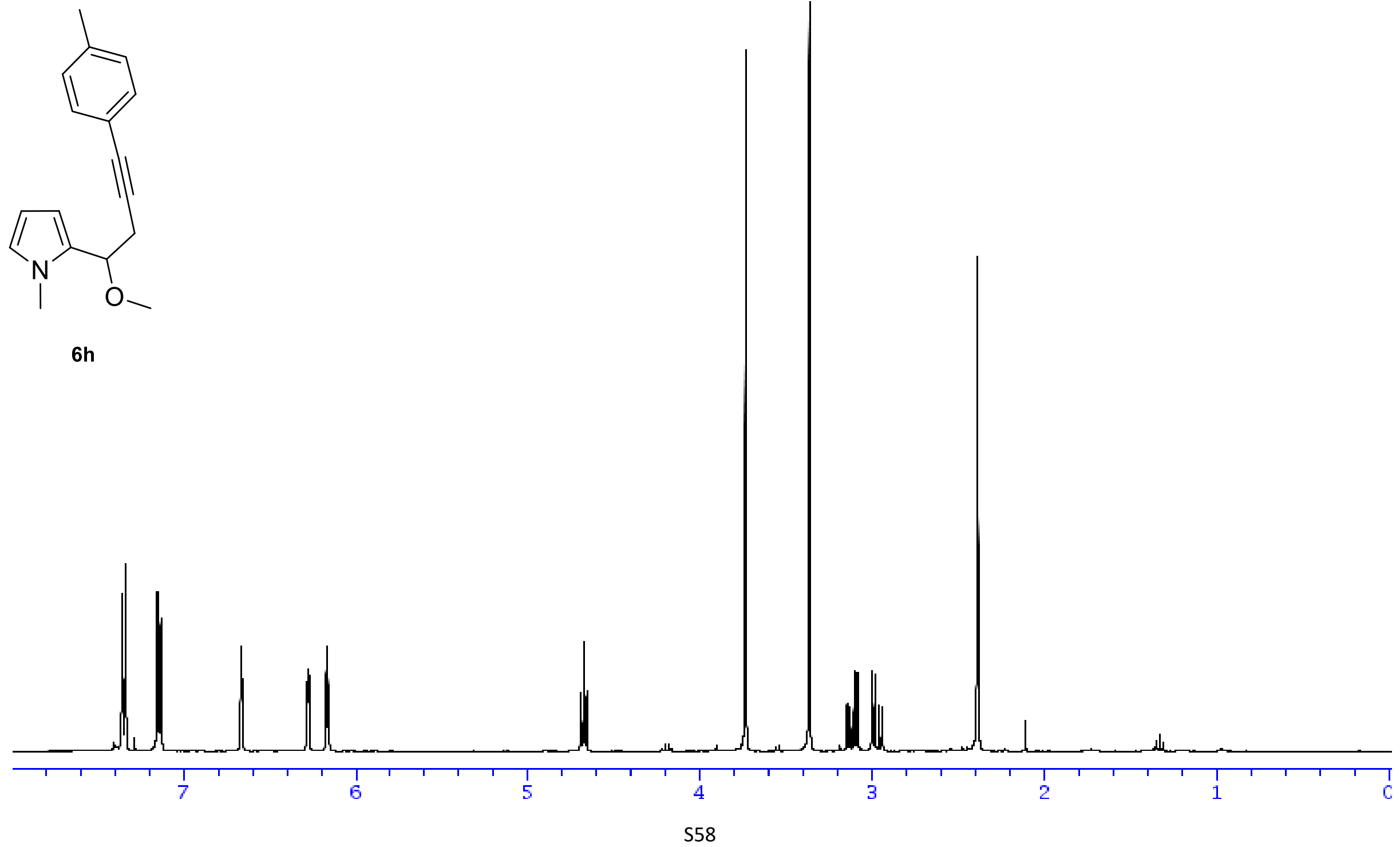
solvent: $\langle\mathrm{CDCl} 3\rangle$

Frequency. $100.612769 \mathrm{MHz}$

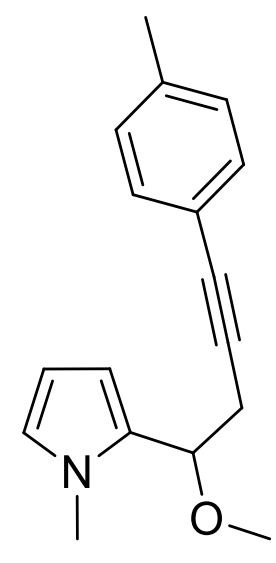

$6 h$

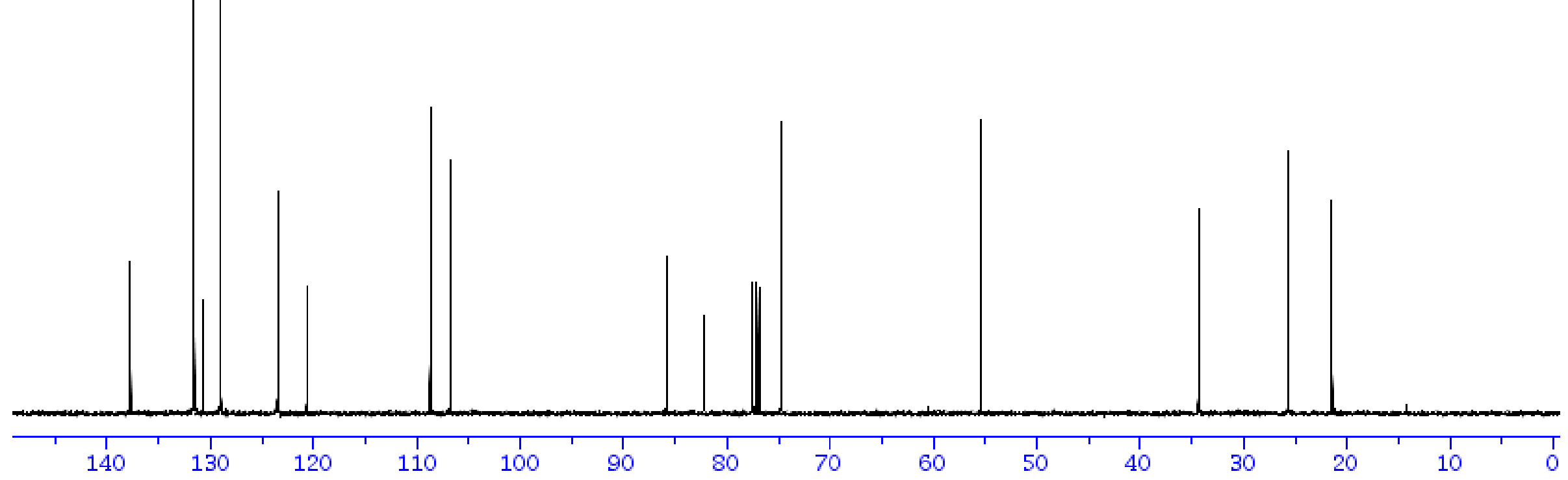




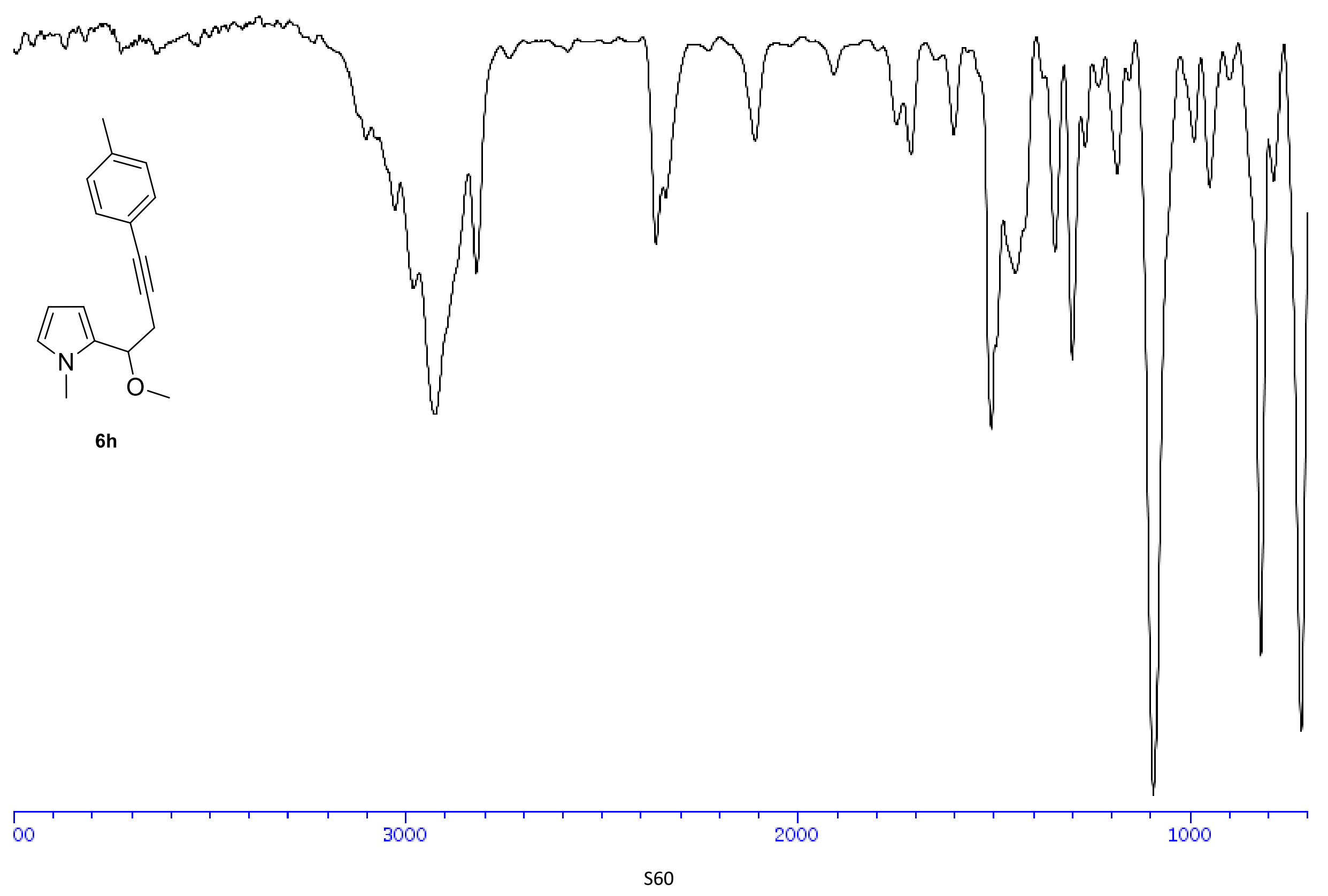


solvent:<CDCI3>

Frequency: $400.13 \mathrm{MHz}$

OMe

6i

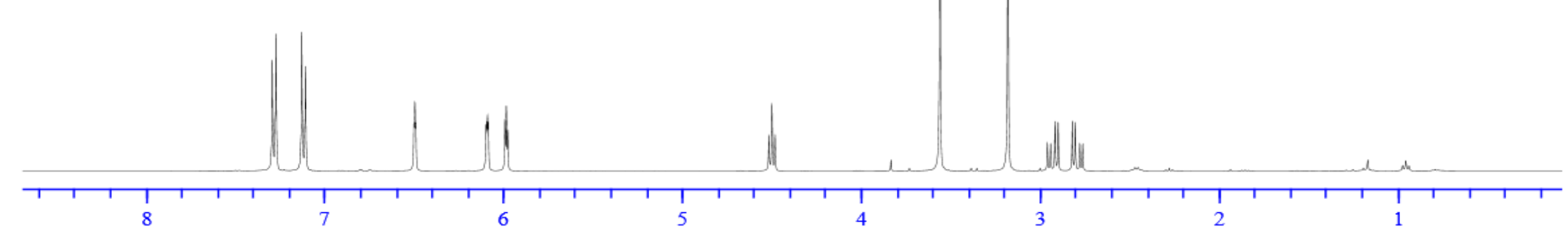


solvent:<CDCI3>

Frequency: $100.612769 \mathrm{MHz}$
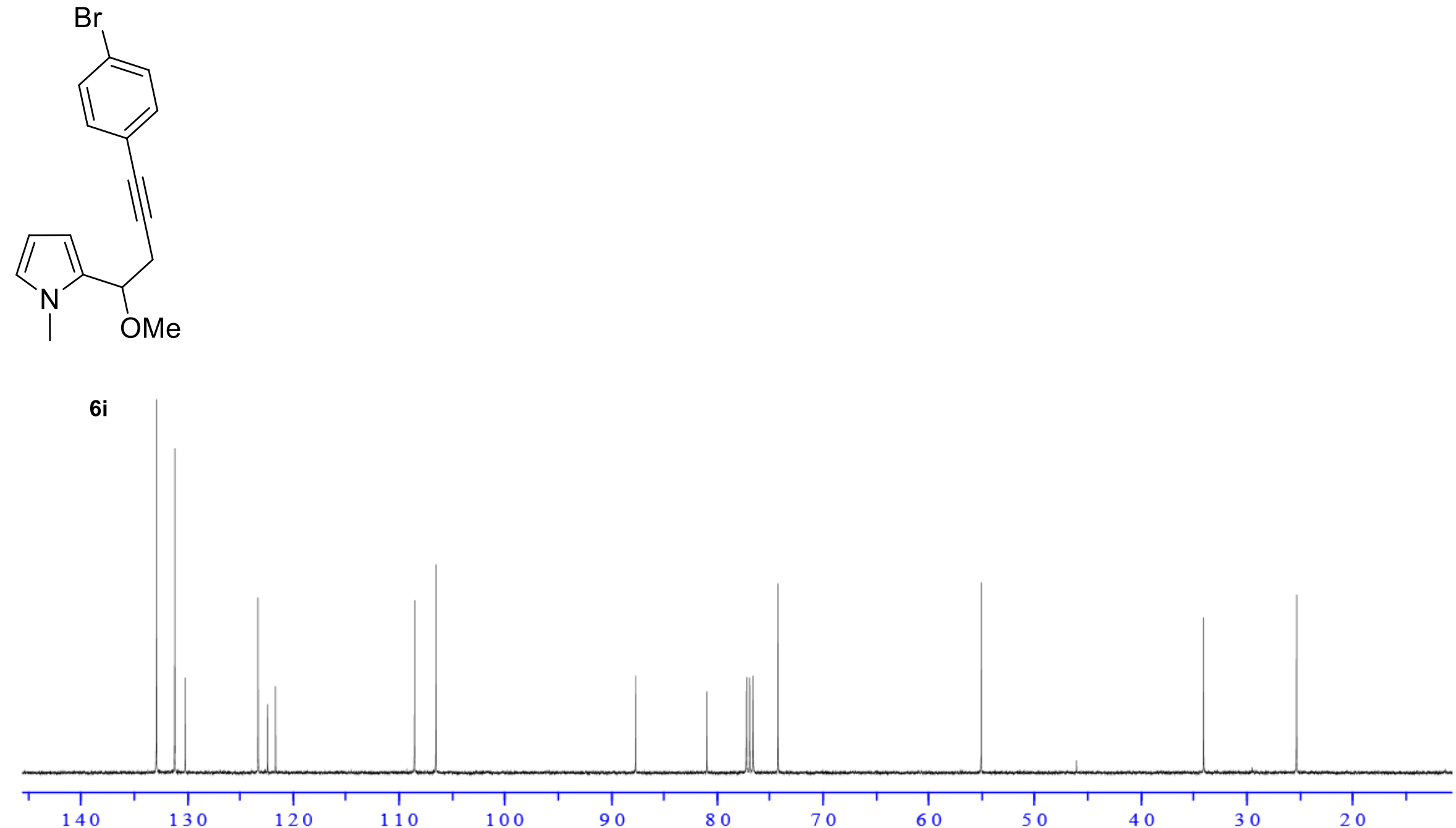


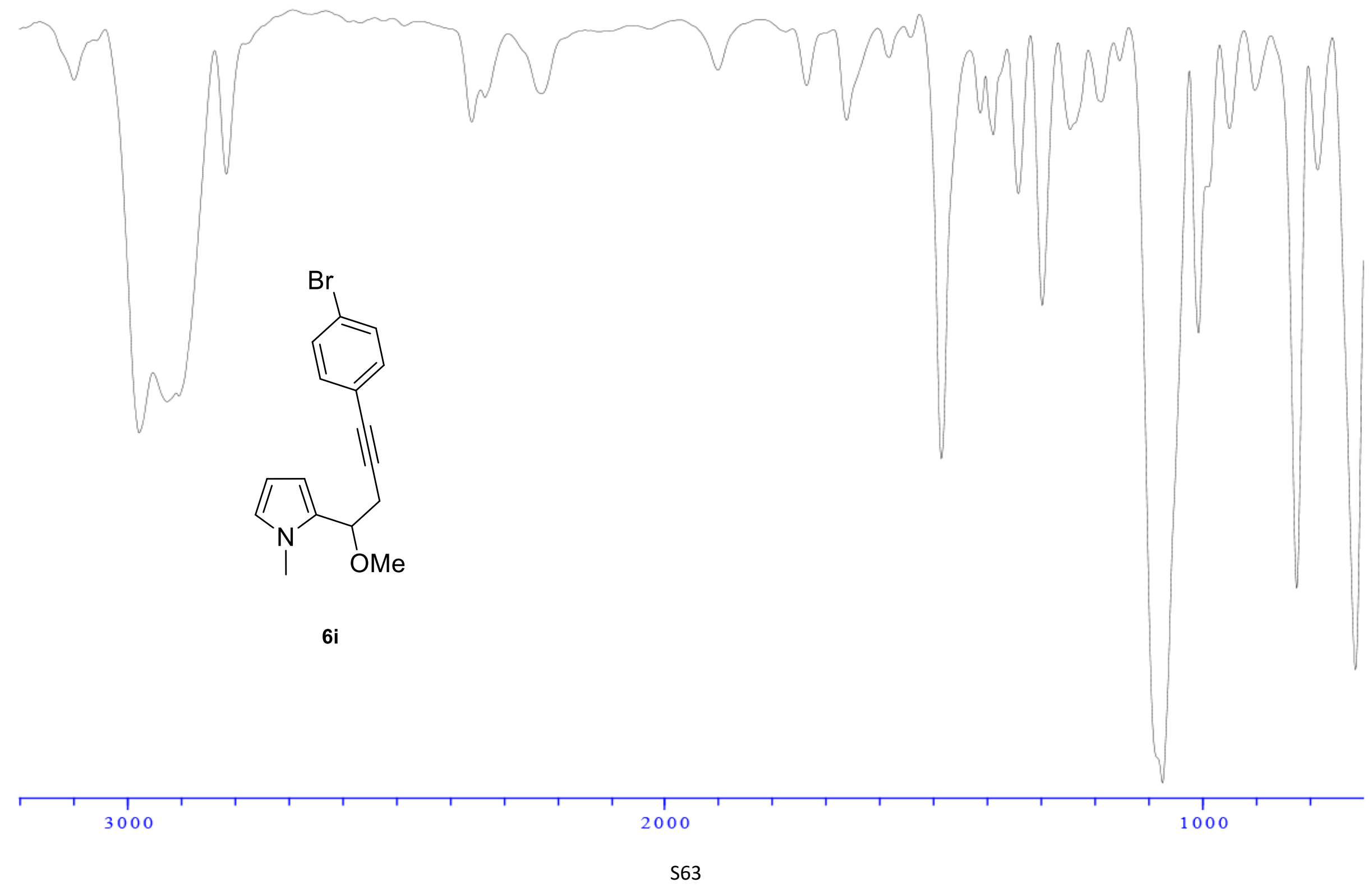


solvent: $\langle\mathrm{CDCI} 3\rangle$

Frequency. $400.13 \mathrm{MHz}$

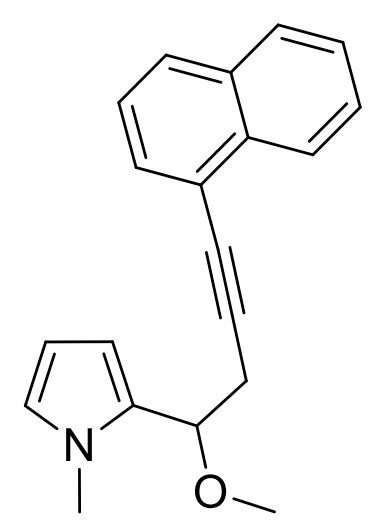

6j

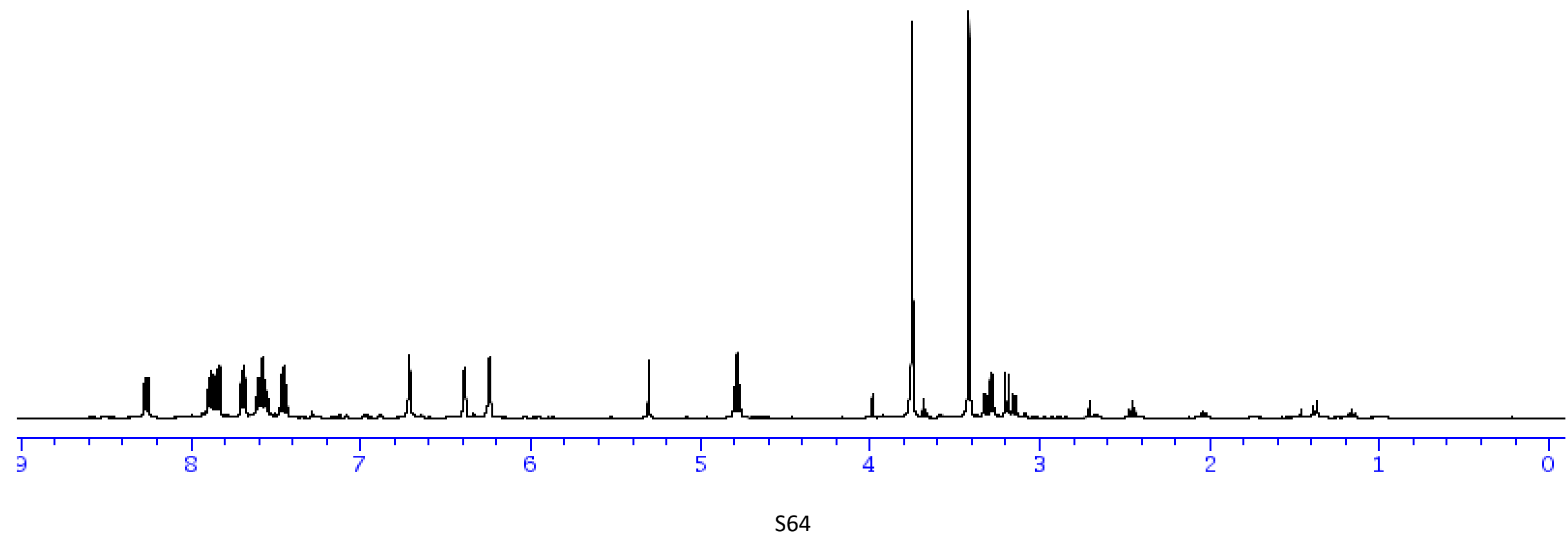


solvent: $<\mathrm{CDCI}$ >

Frequency $100.612769 \mathrm{MHz}$
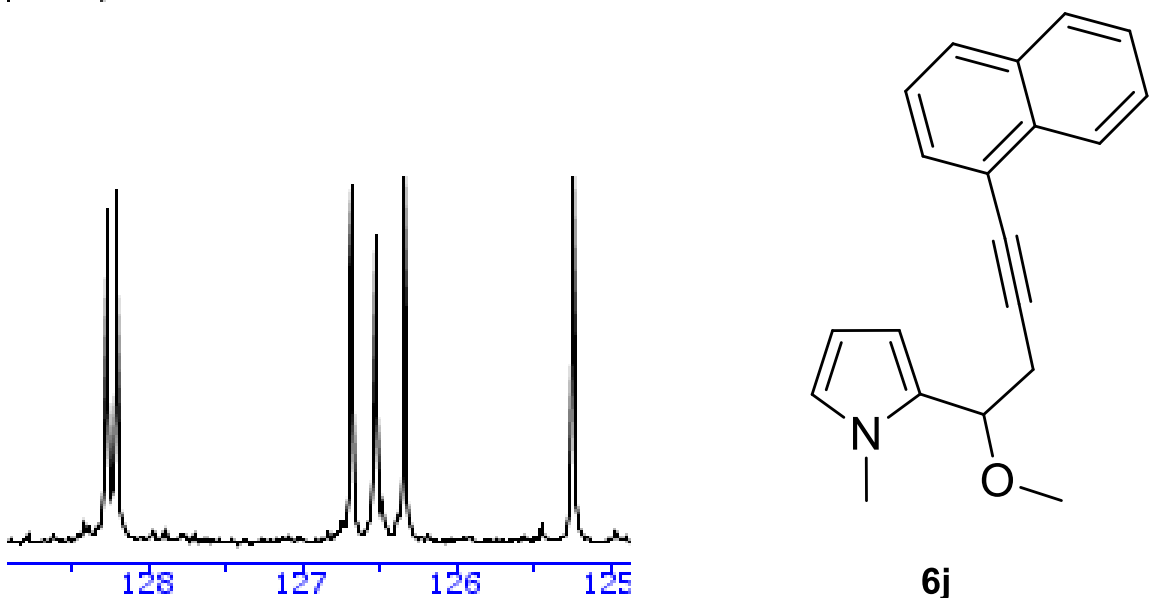

6j

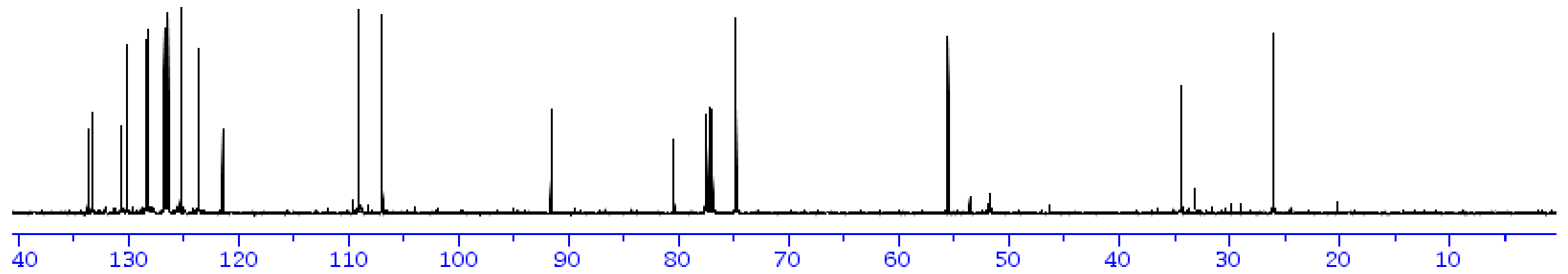




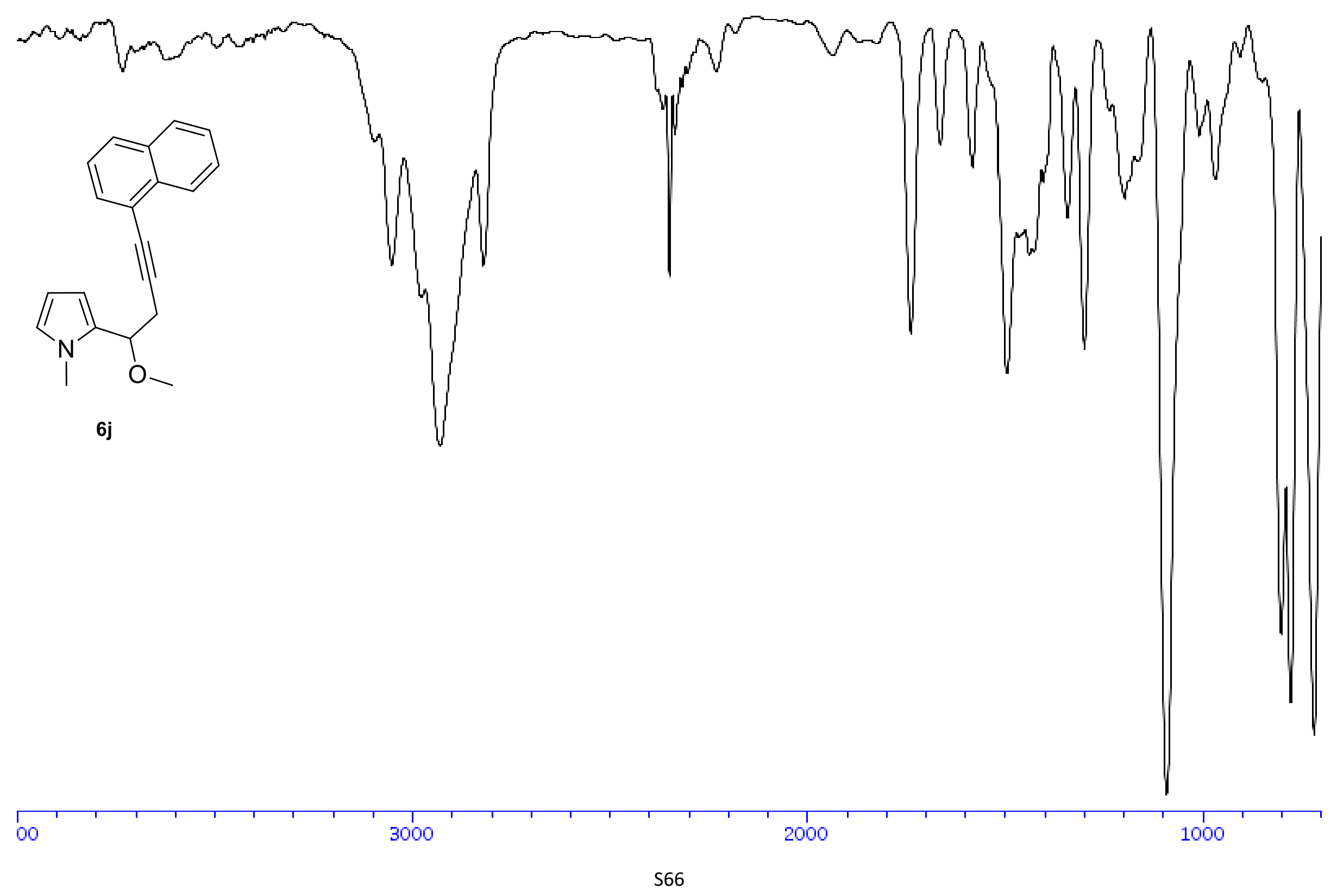


solvent: $\langle\mathrm{CDCI} 3\rangle$

Frequency. $400.13 \mathrm{MHz}$

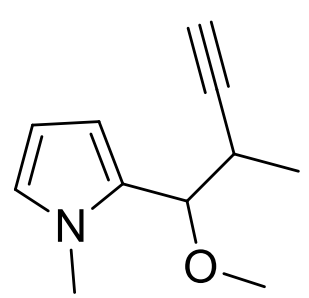

$6 k$

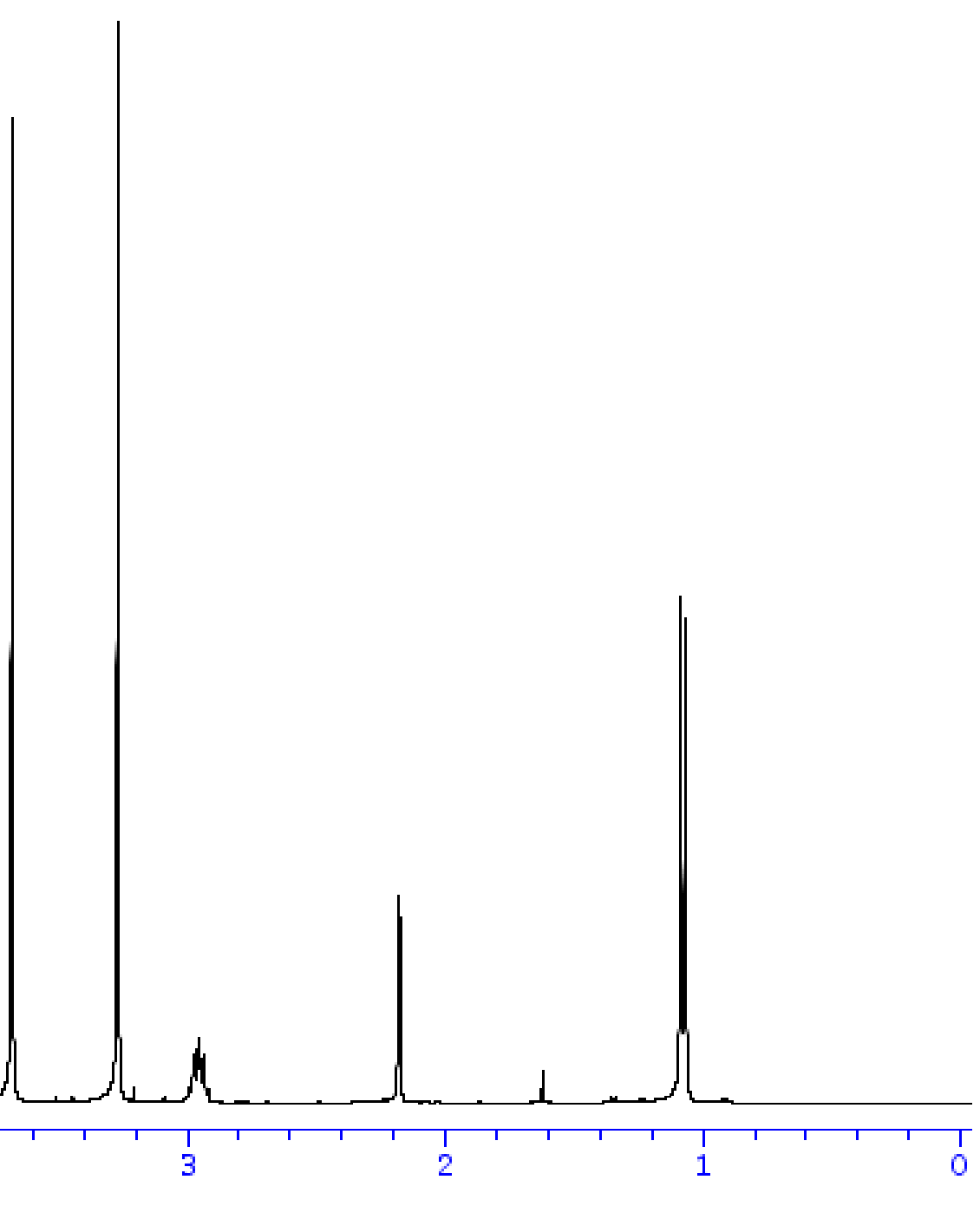


solvent: $\langle\mathrm{CDCI} 3\rangle$

Frequency. 100.612769M Hz

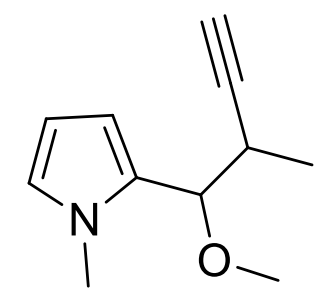

$6 k$

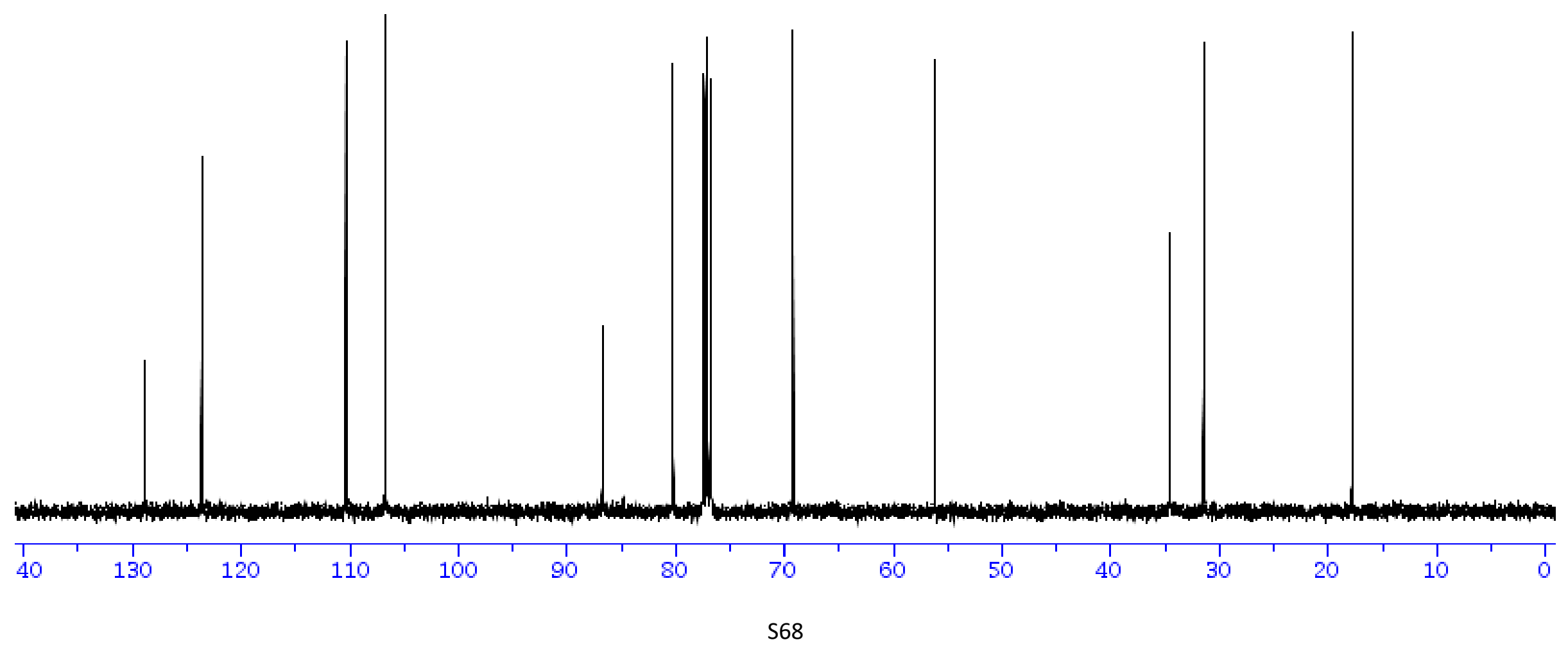




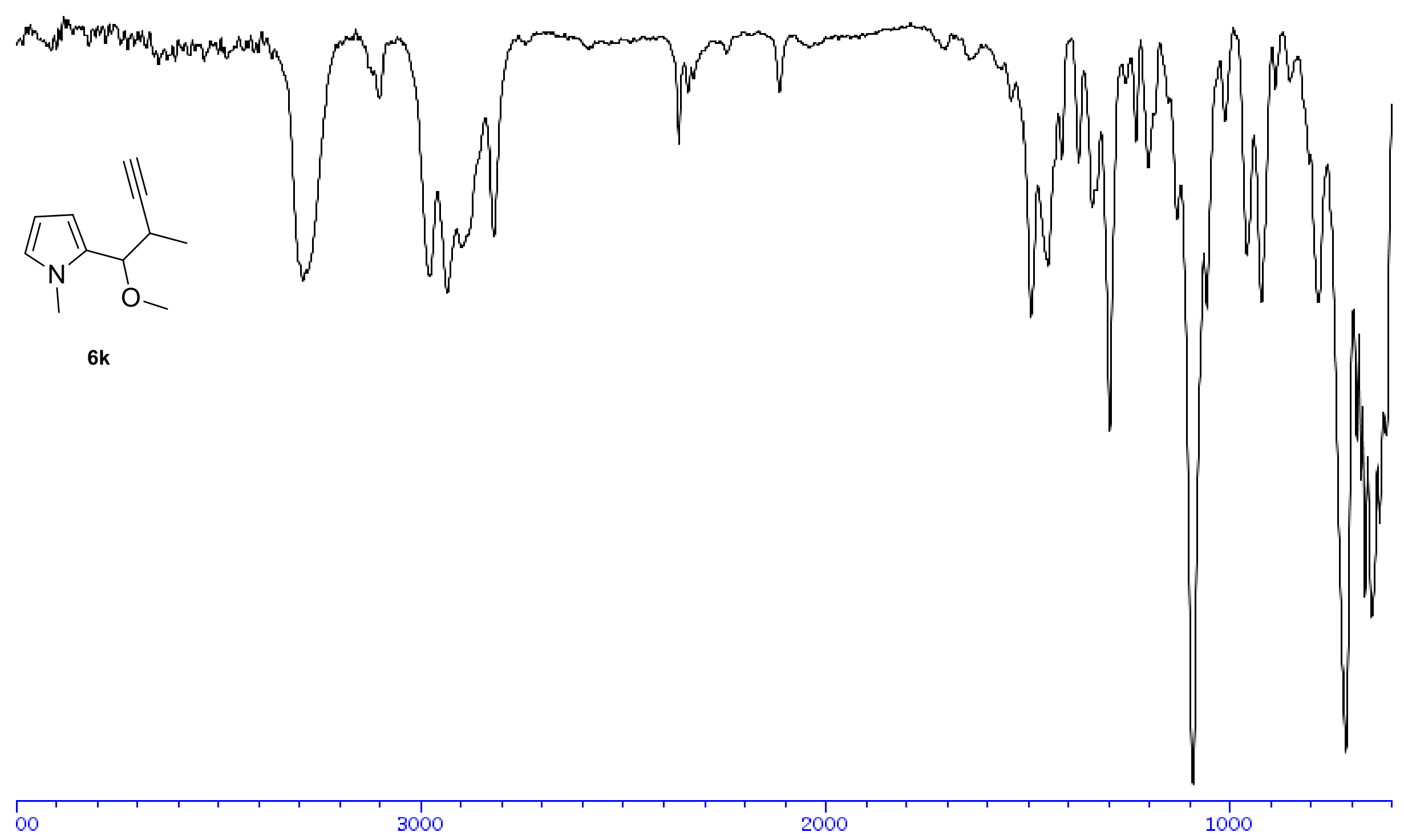


solvent: $<\mathrm{CDCI} 3>$

Frequency. $400.13 \mathrm{MHz}$

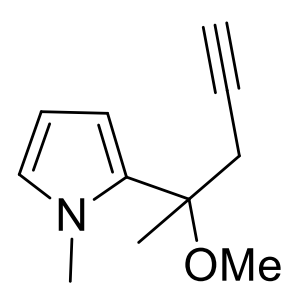

61

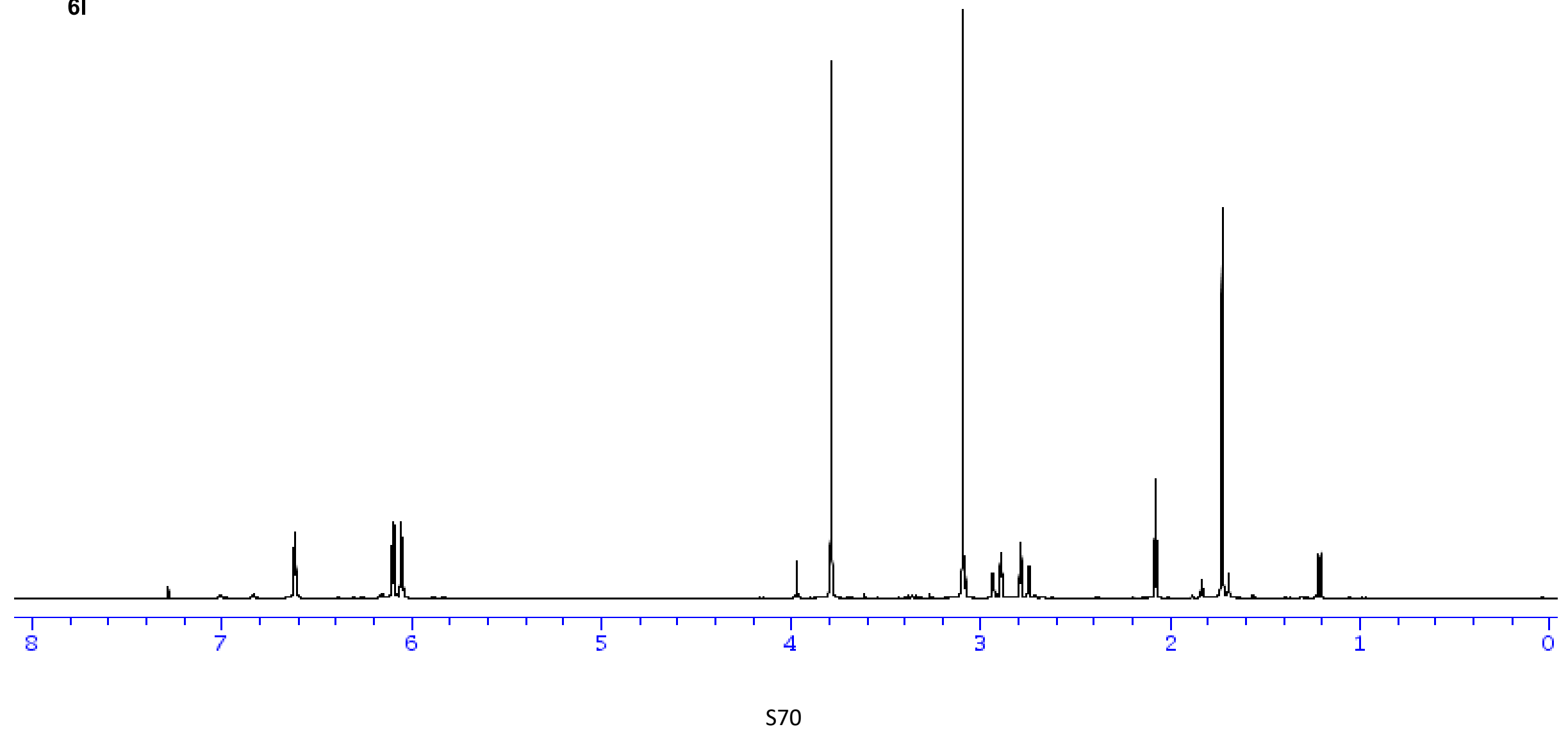


solvent: $<C D C I 3>$

Frequency. 100.612769MHz

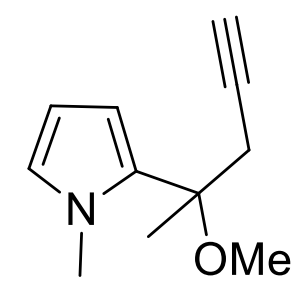

6I

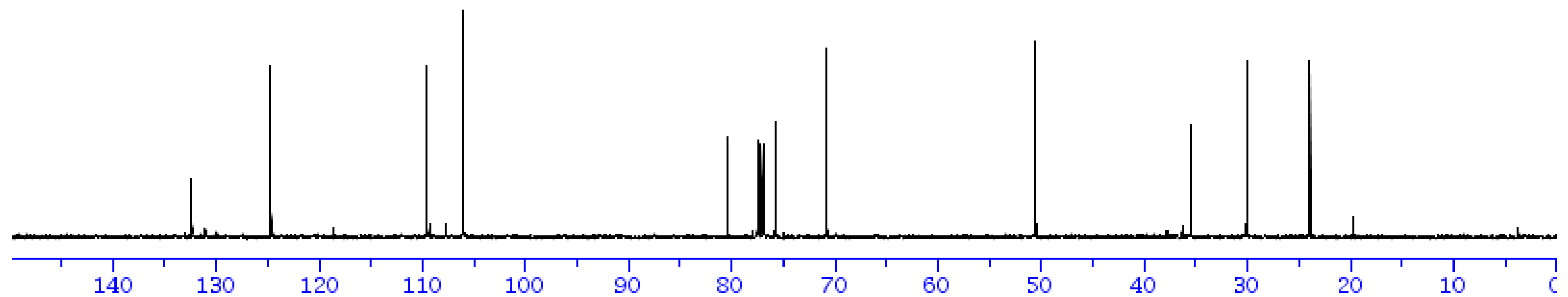




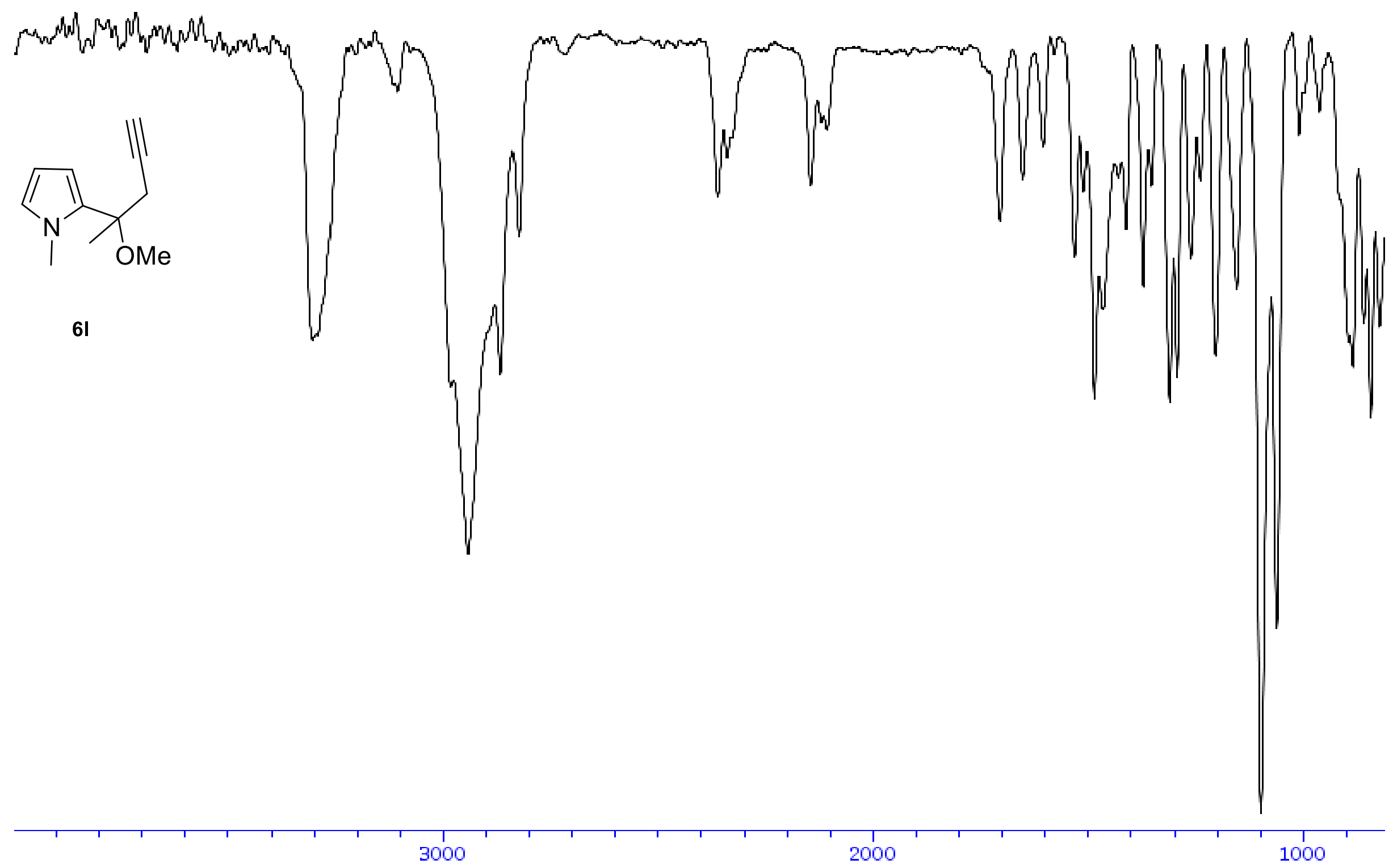


solvent: $<\mathrm{CDCI}>$

Frequency. 400. $13 \mathrm{MHz}$

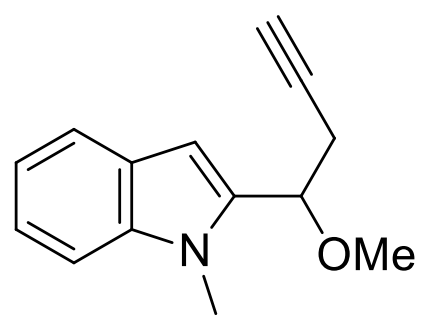

$6 m$

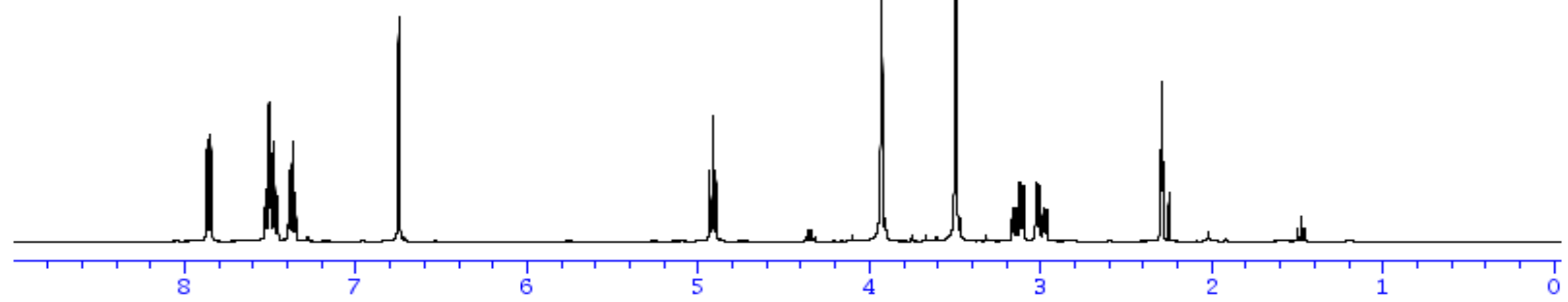

S73 
solvent: $<\mathrm{CDCI} 3>$

Frequency. $100.612769 \mathrm{MHz}$

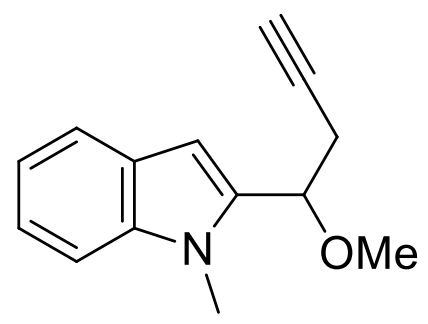

$6 m$

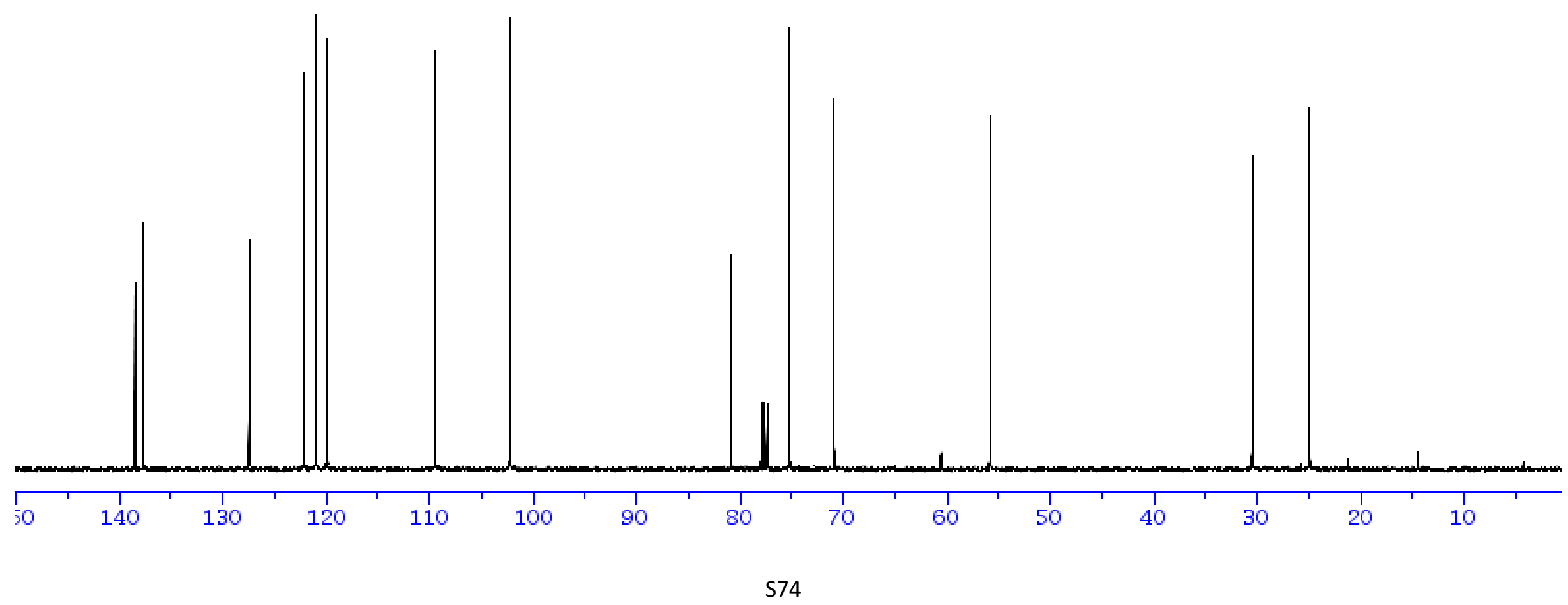




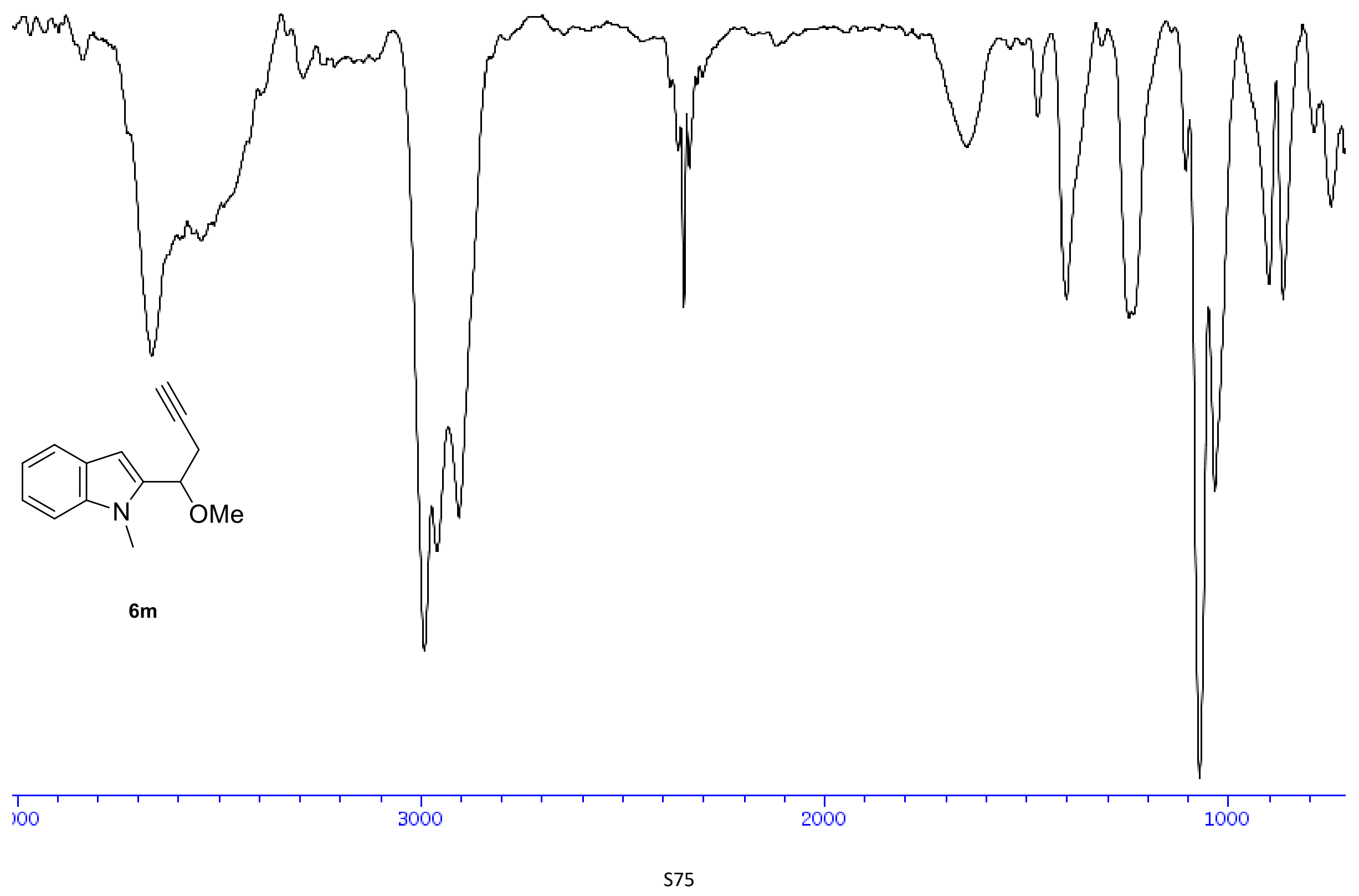


solvent: $<\mathrm{CDCI} 3>$

Frequency. $400.13 \mathrm{MHz}$

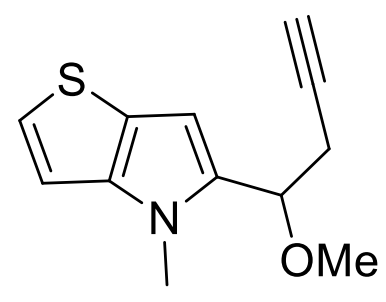

$6 n$

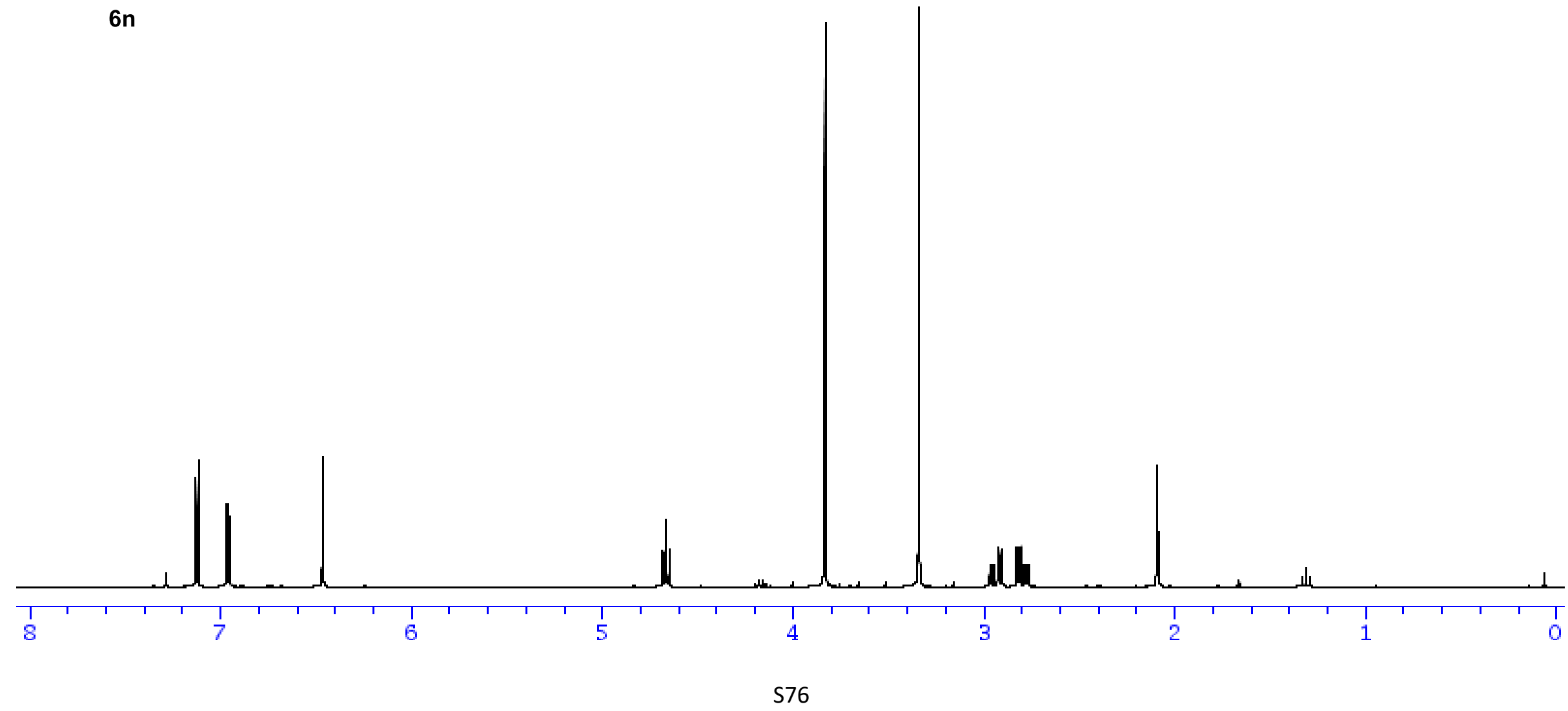


solvent: $\angle \mathrm{CDCI} 3>$

Frequency. $100.612769 \mathrm{MHz}$

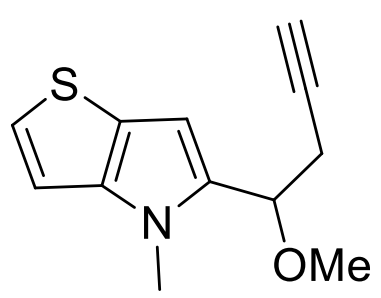

$6 n$

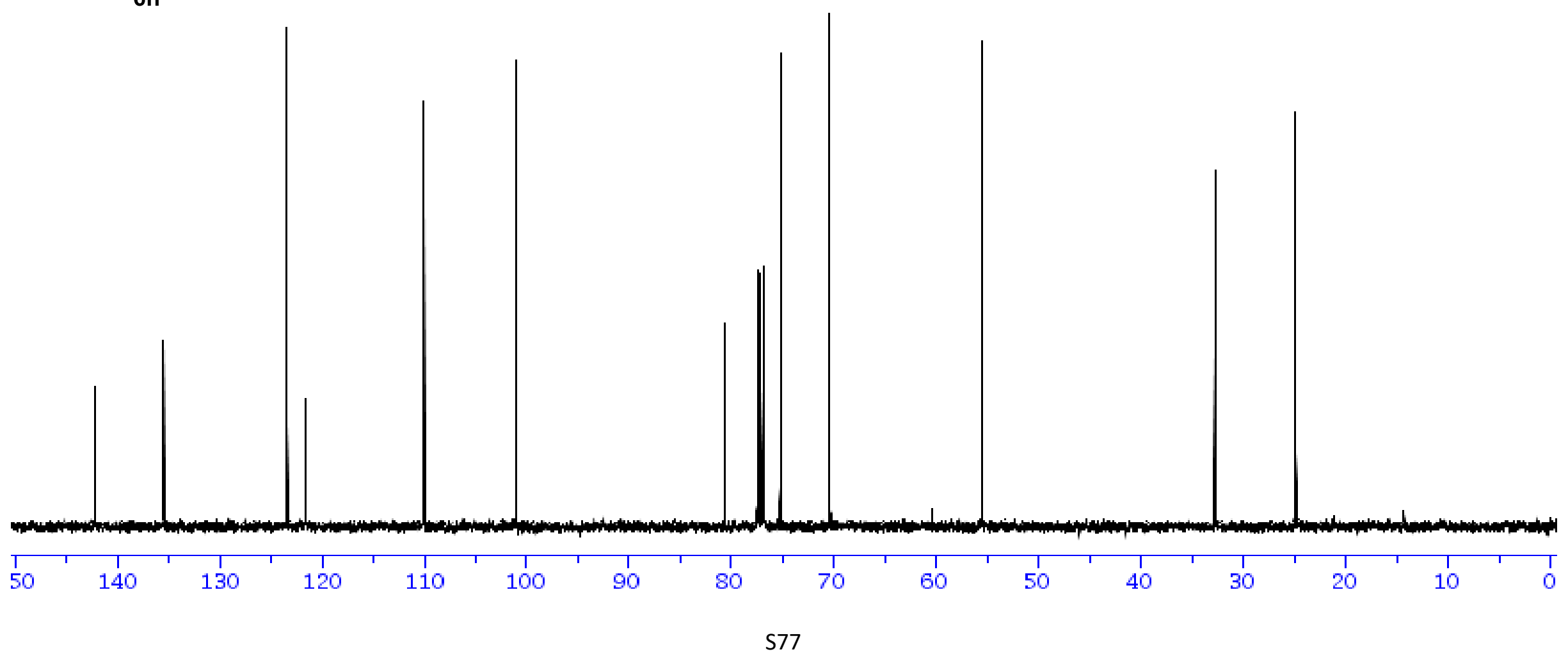




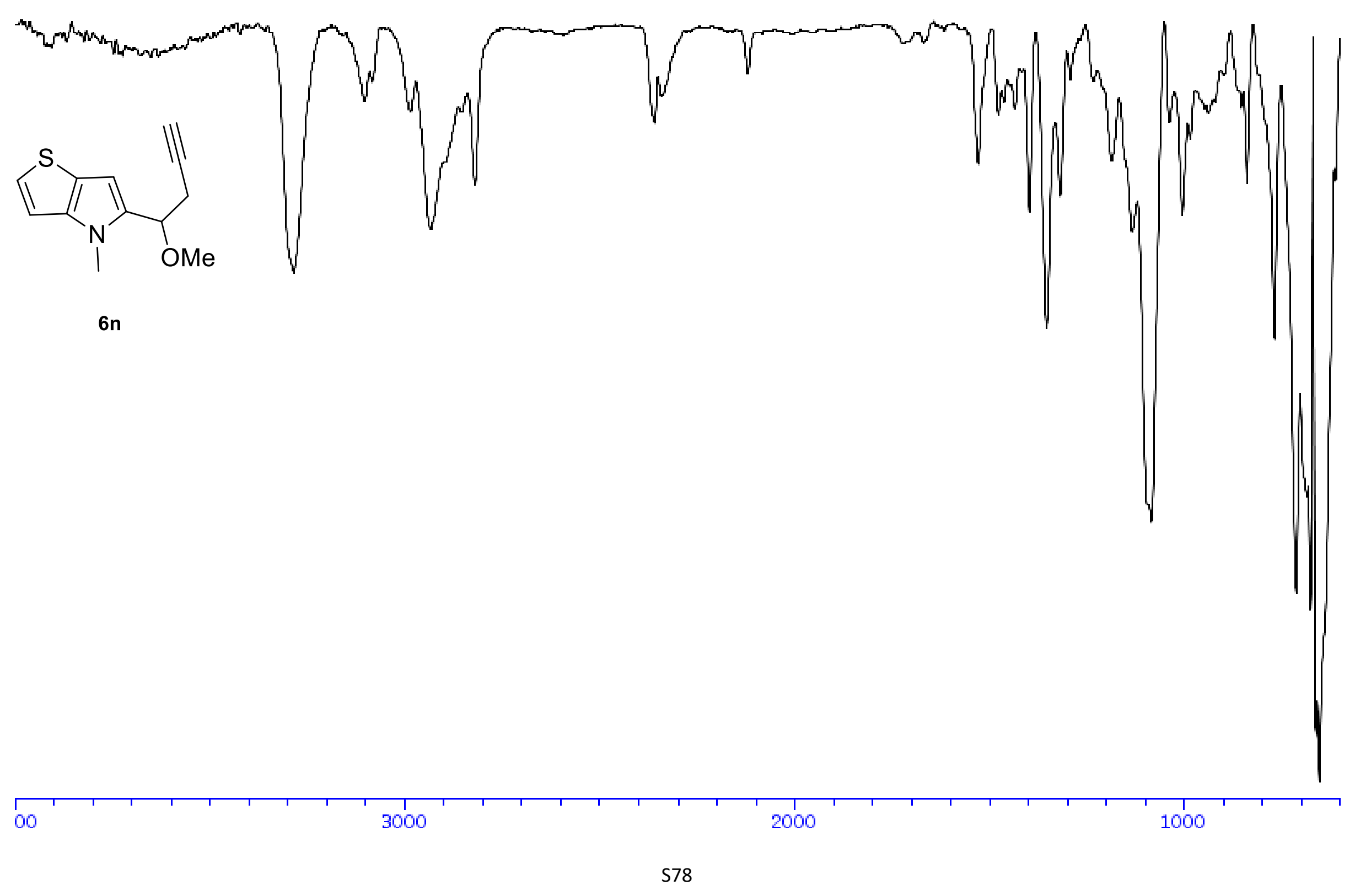


solvent: $<\mathrm{CDCI}$ >

Frequency. $400.13 \mathrm{MHz}$<smiles>C#CCC(OC)c1ccn(C)c1</smiles>

$9 a$

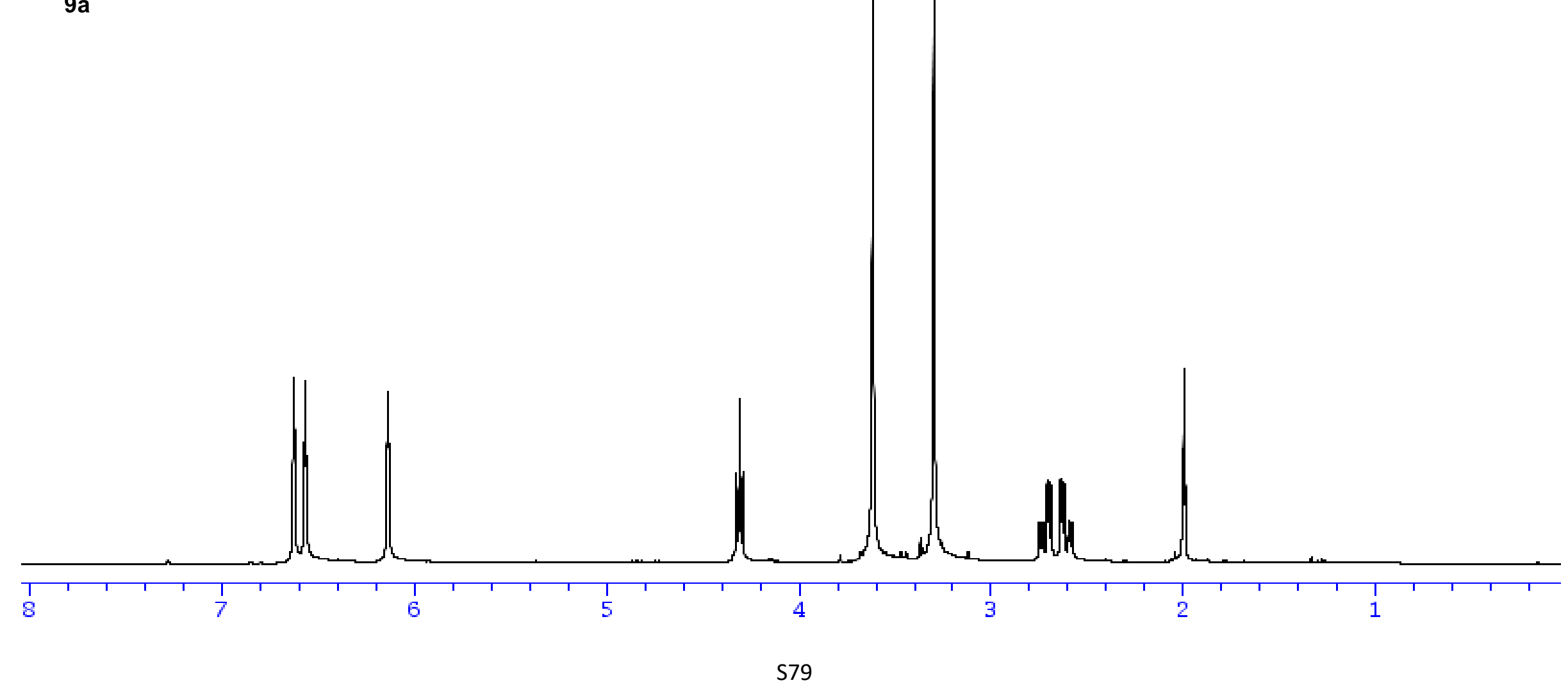


solvent: $\langle\mathrm{CDCl} 3>$

Frequency. 100.612769M Hz

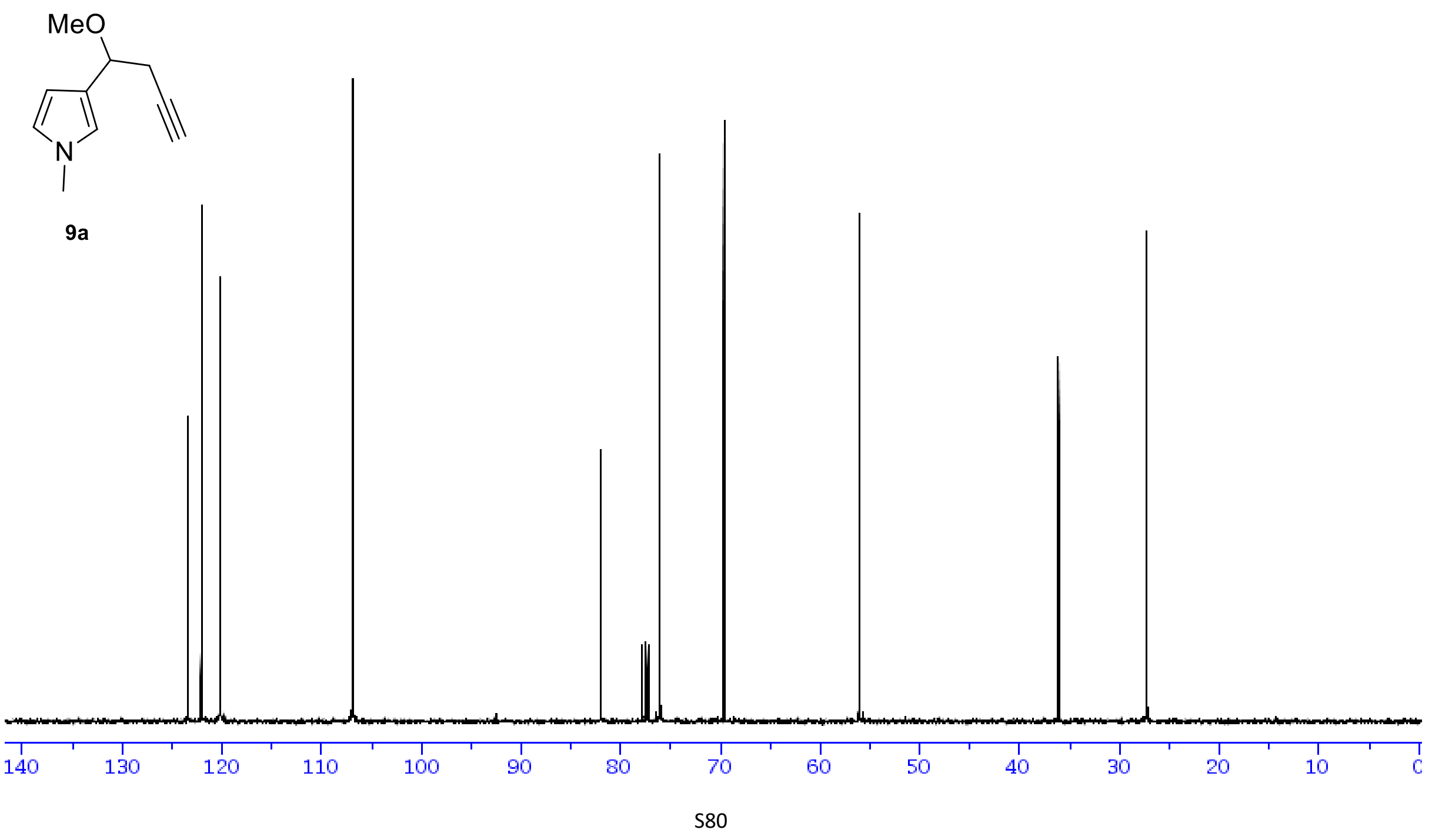




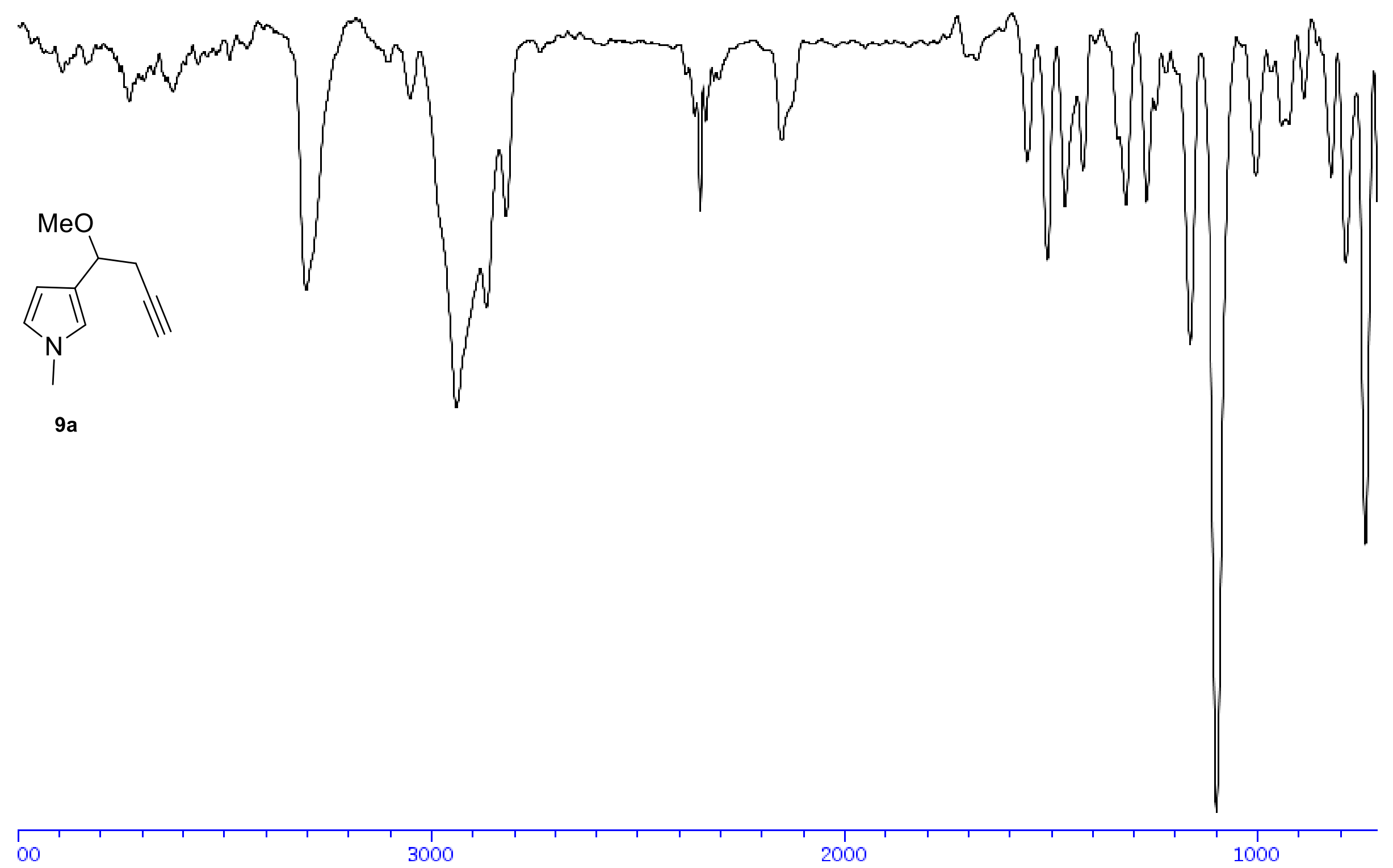


solvent: $\langle\mathrm{CDCI} 3\rangle$

Frequency. $400.13 \mathrm{MHz}$

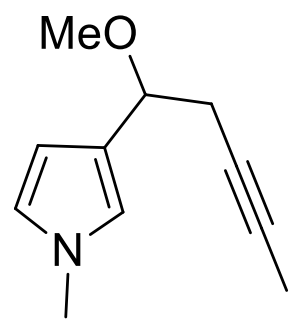

$9 b$

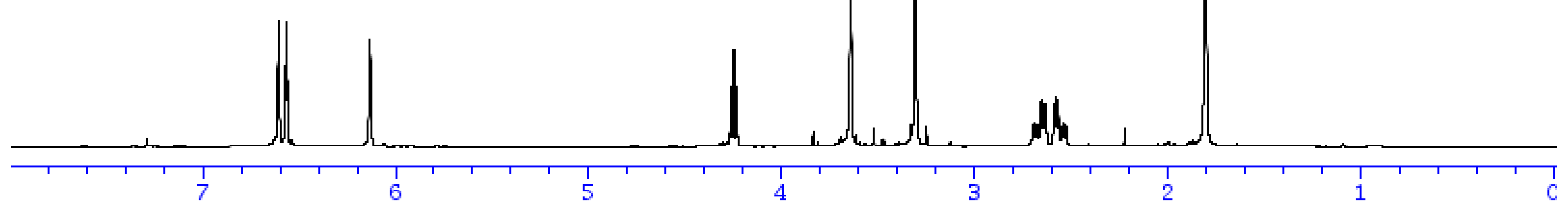

S82 
solvent: $<\mathrm{CDCI} 3>$

Frequency. $100.612769 \mathrm{MHz}$

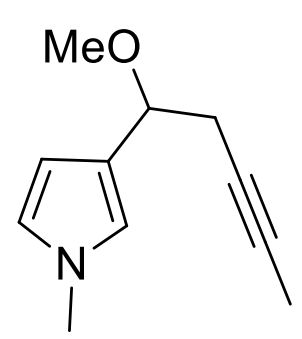

$9 b$

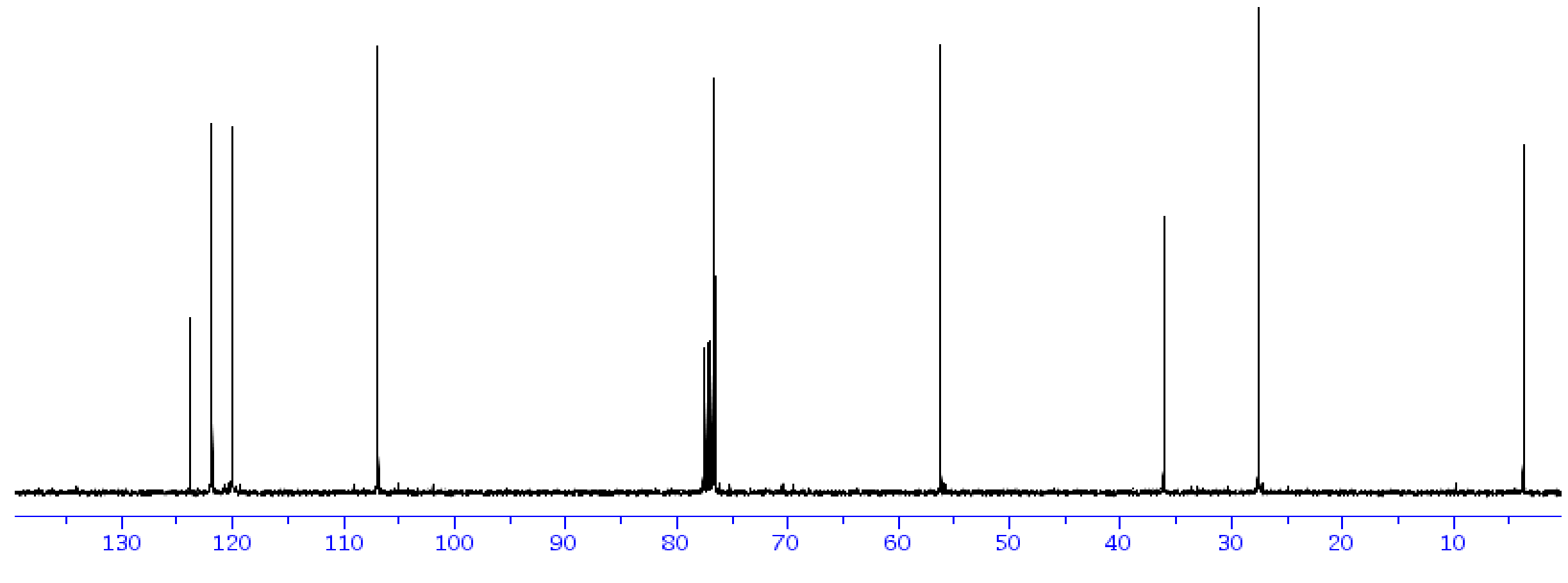




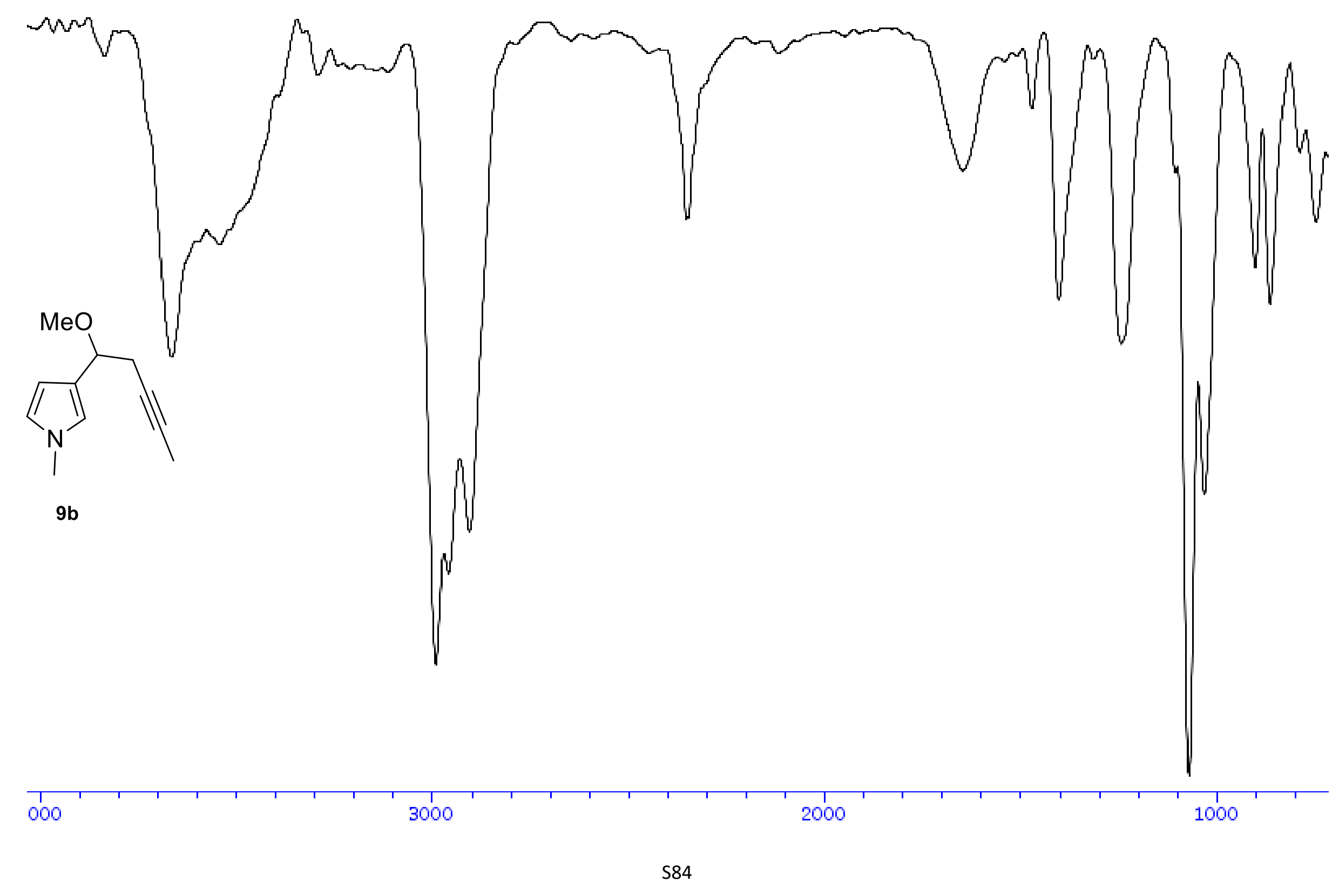


solvent: $\langle\mathrm{CDCI} 3>$

Frequency. 400.13 MHz

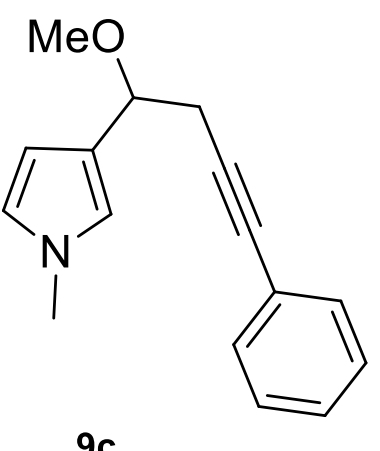

9c

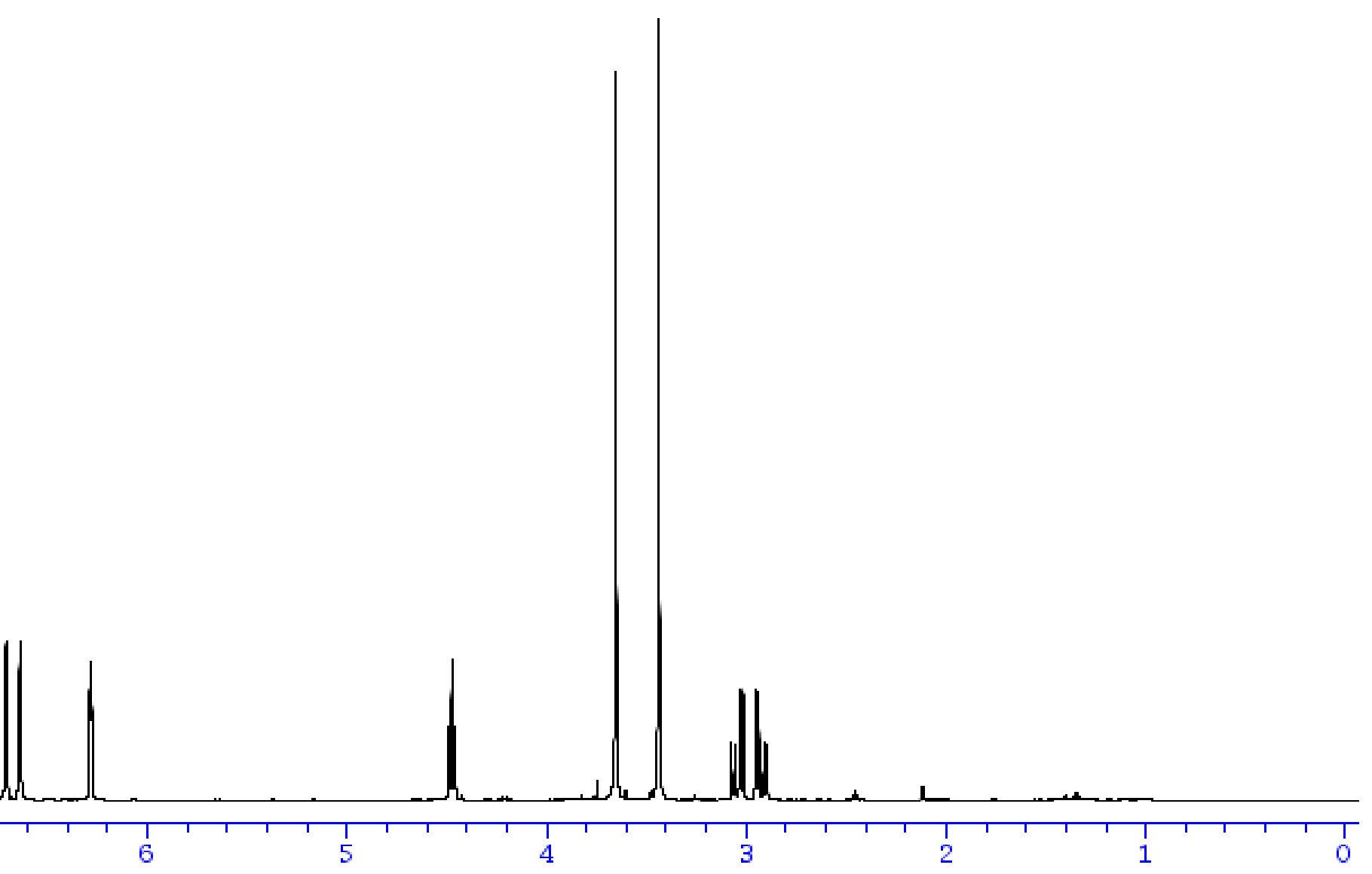


solvent: $\angle \mathrm{CDCl} 3\rangle$

Frequency. $100.612769 \mathrm{MHz}$

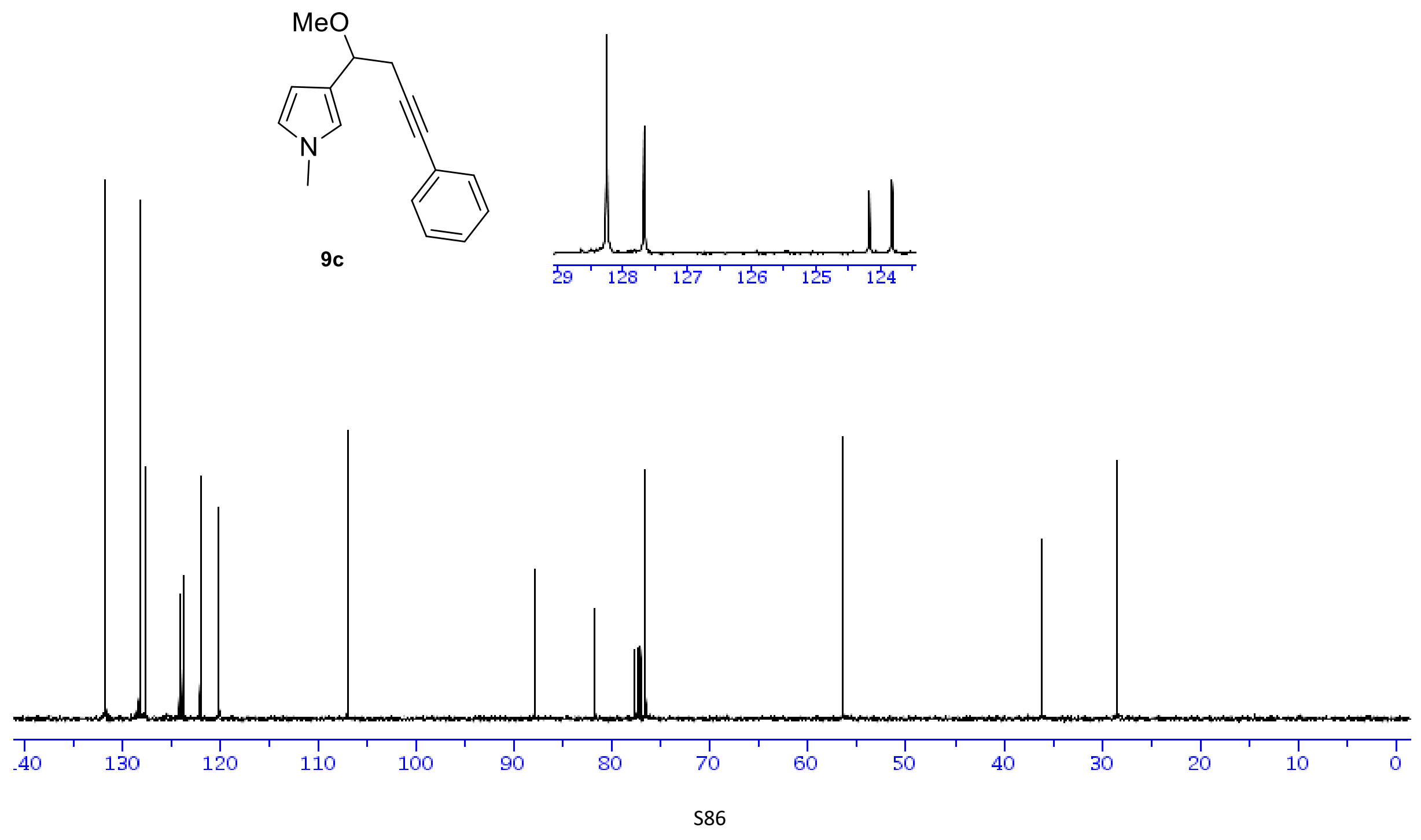




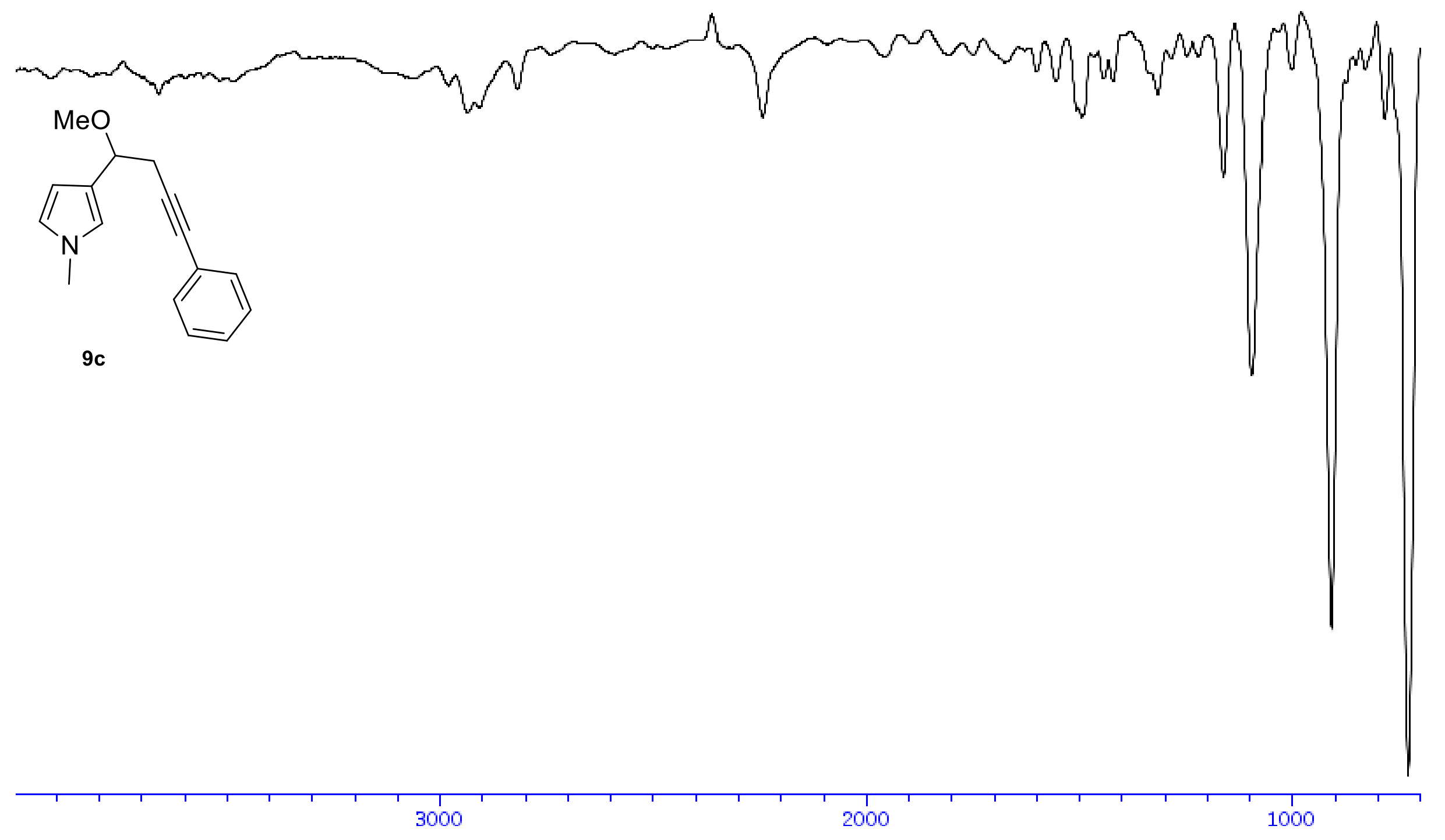


solvent: $\langle\mathrm{CDCI} 3\rangle$

Frequency. $400.13 \mathrm{MHz}$
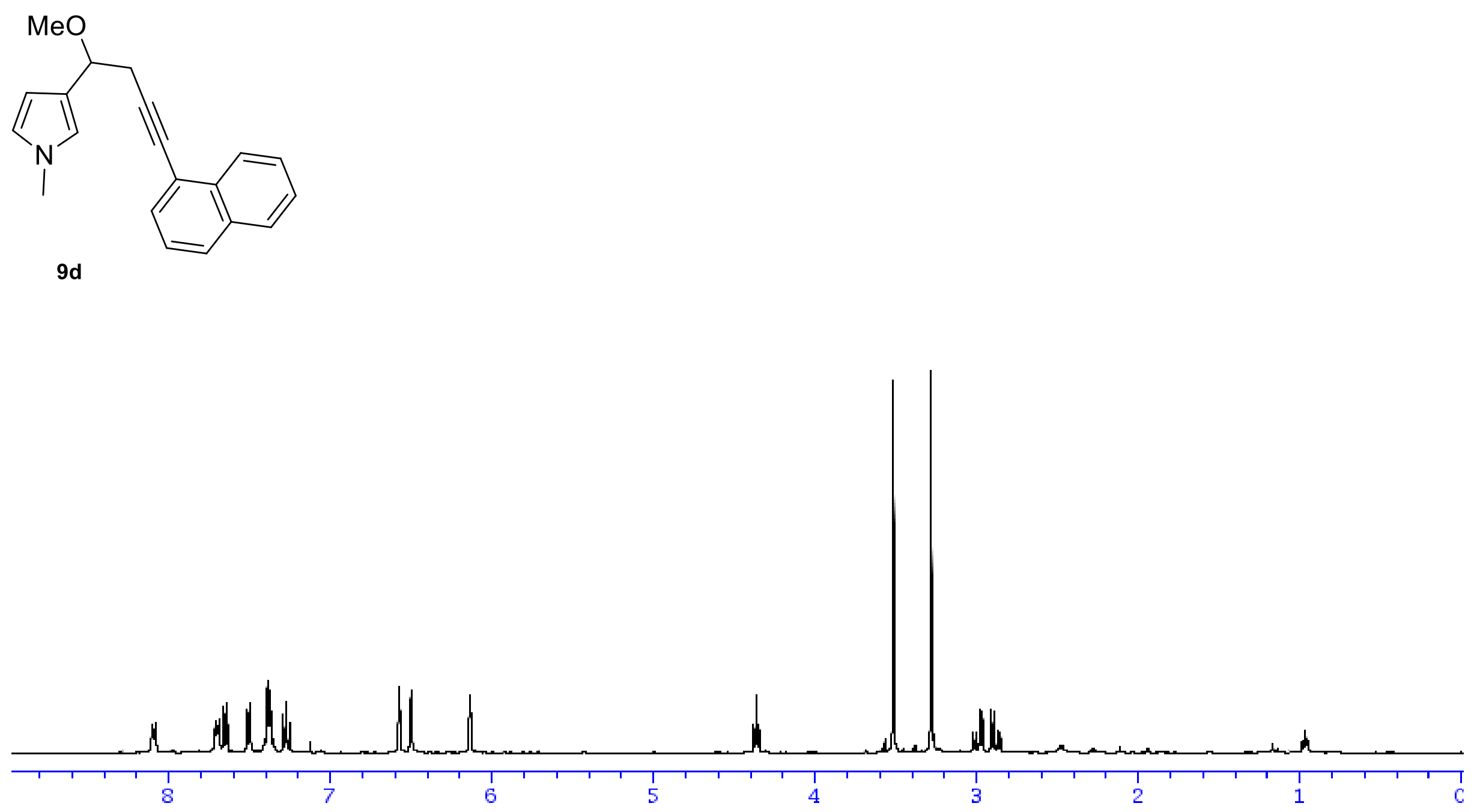
solvent: $\langle\mathrm{CDCI}>$

Frequency. $100.612769 \mathrm{MHz}$
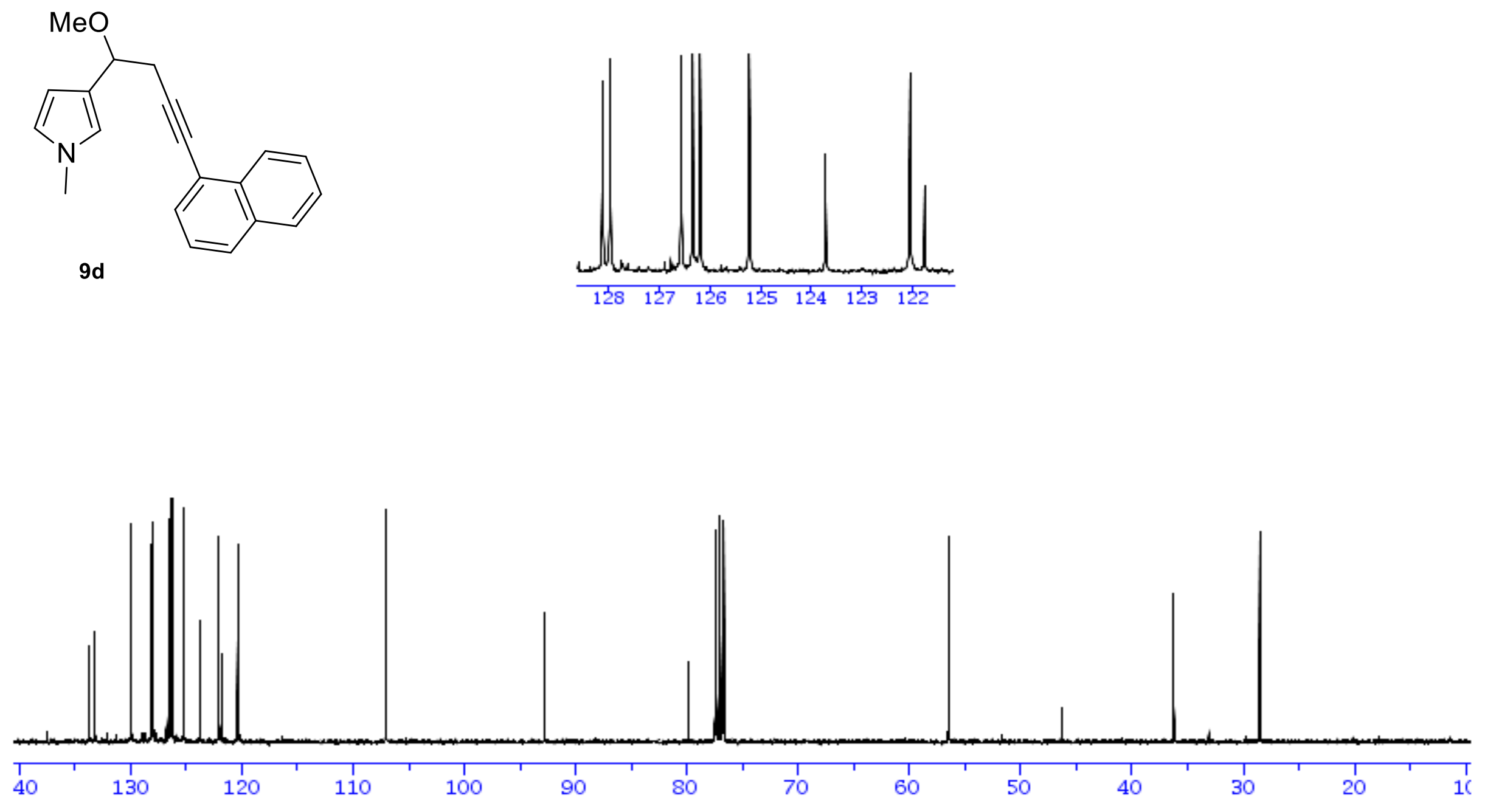


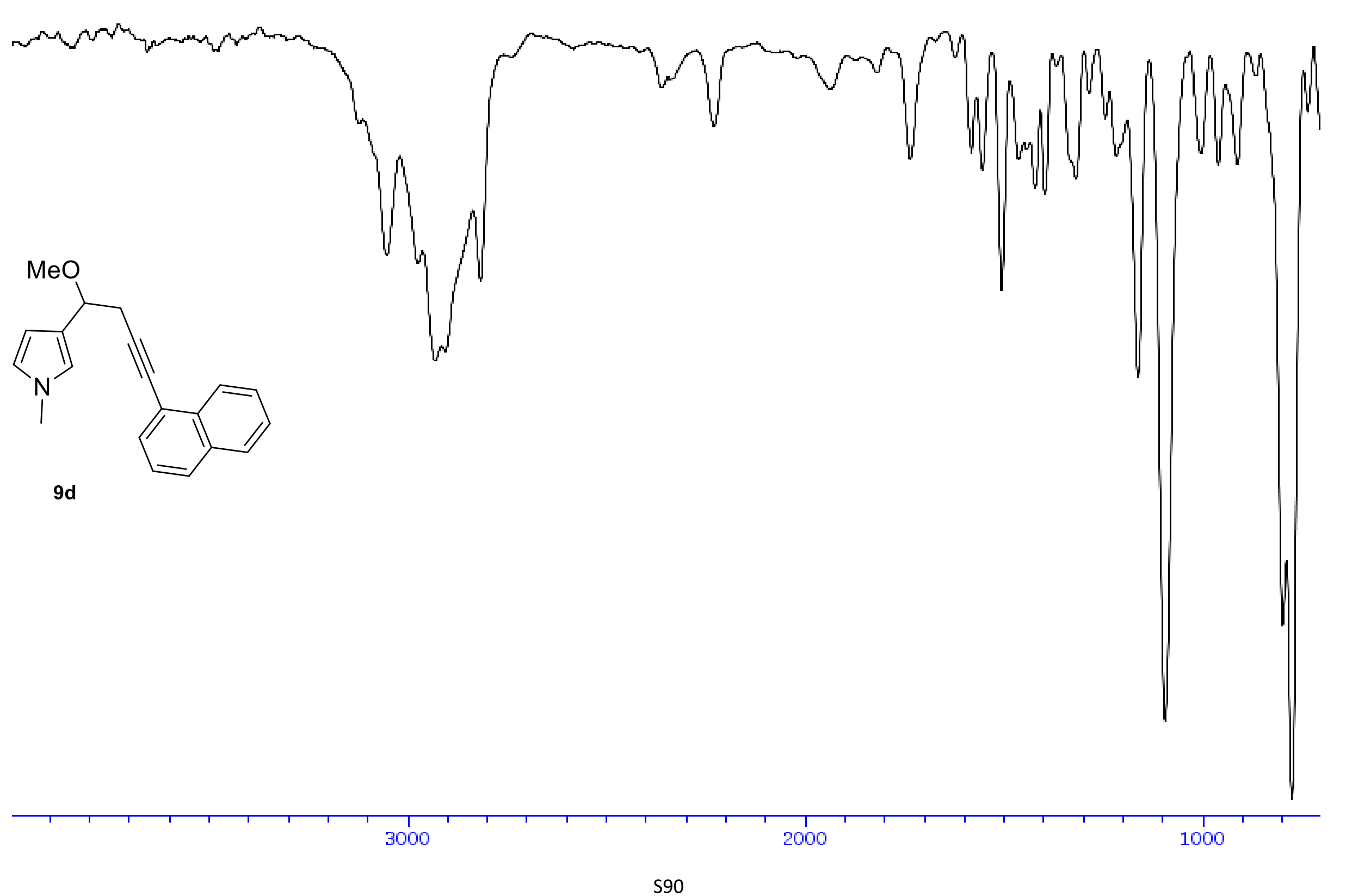


solvent: $<\mathrm{CDCI} 3>$

Frequency. $400.13 \mathrm{MHz}$

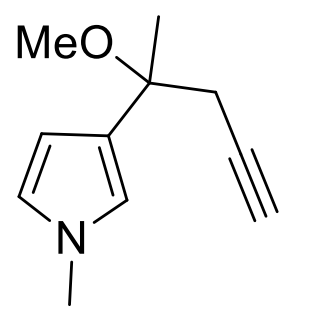

$9 e$

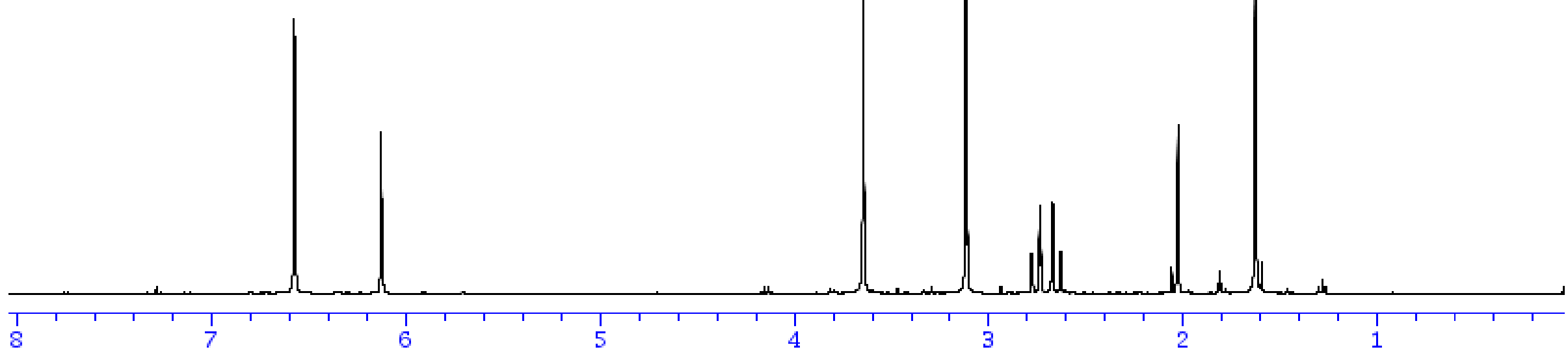

S91 
solvent: $\langle\mathrm{CDCI} 3\rangle$

Frequency. $100.612769 \mathrm{MHz}$

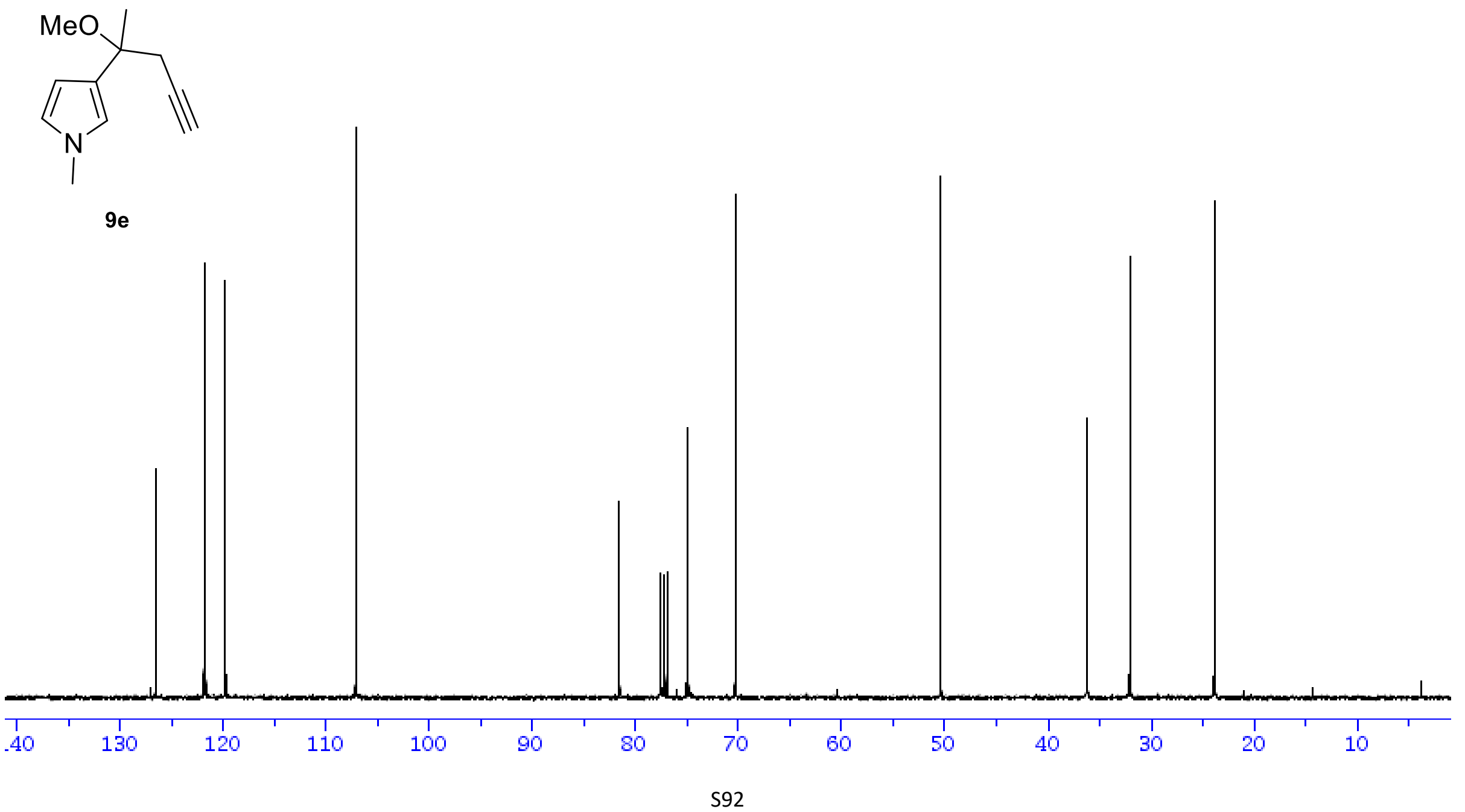



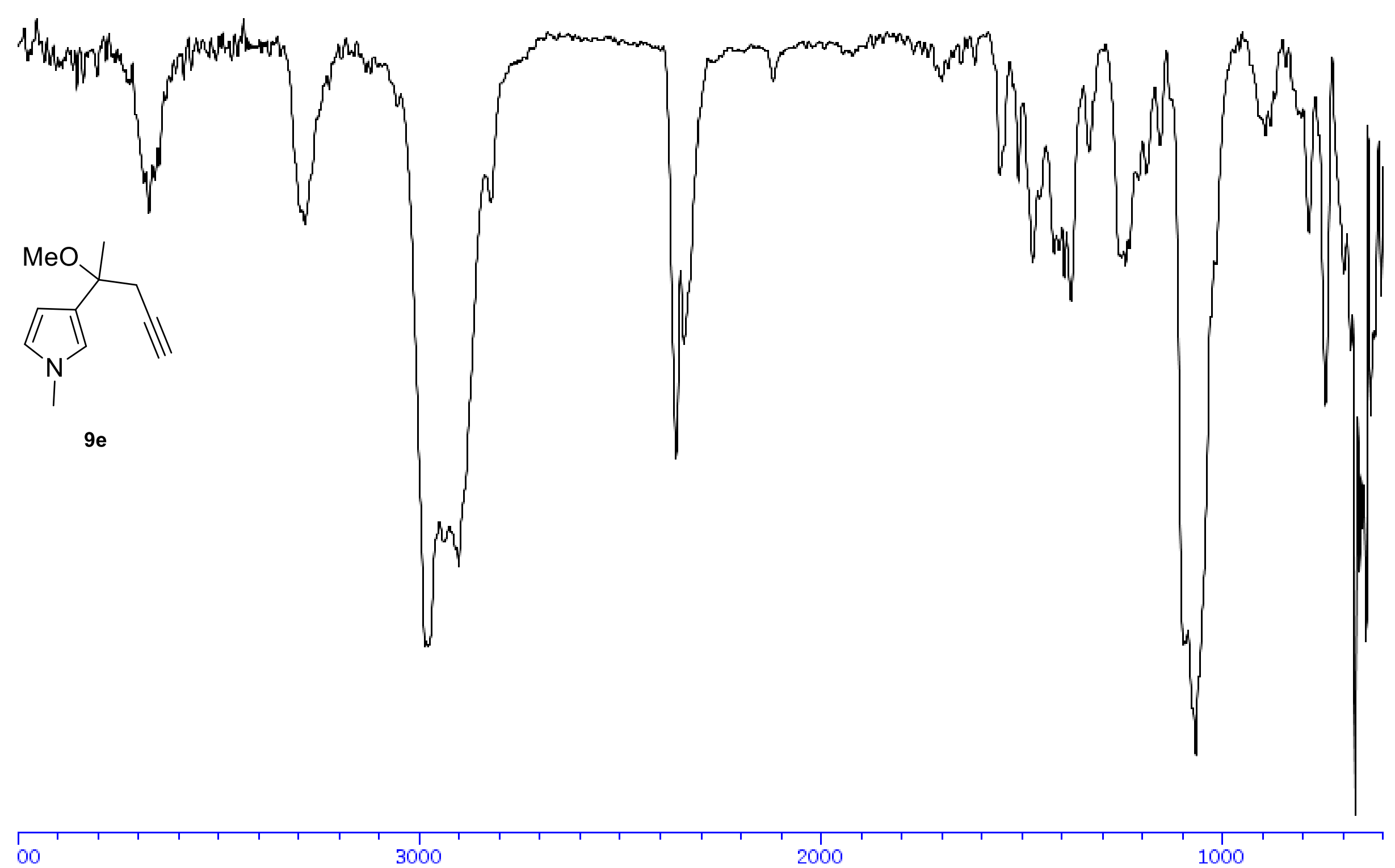

2000

1000 
solvent: $<\mathrm{CDCI} 3>$

Frequency. $400.13 \mathrm{MHz}$
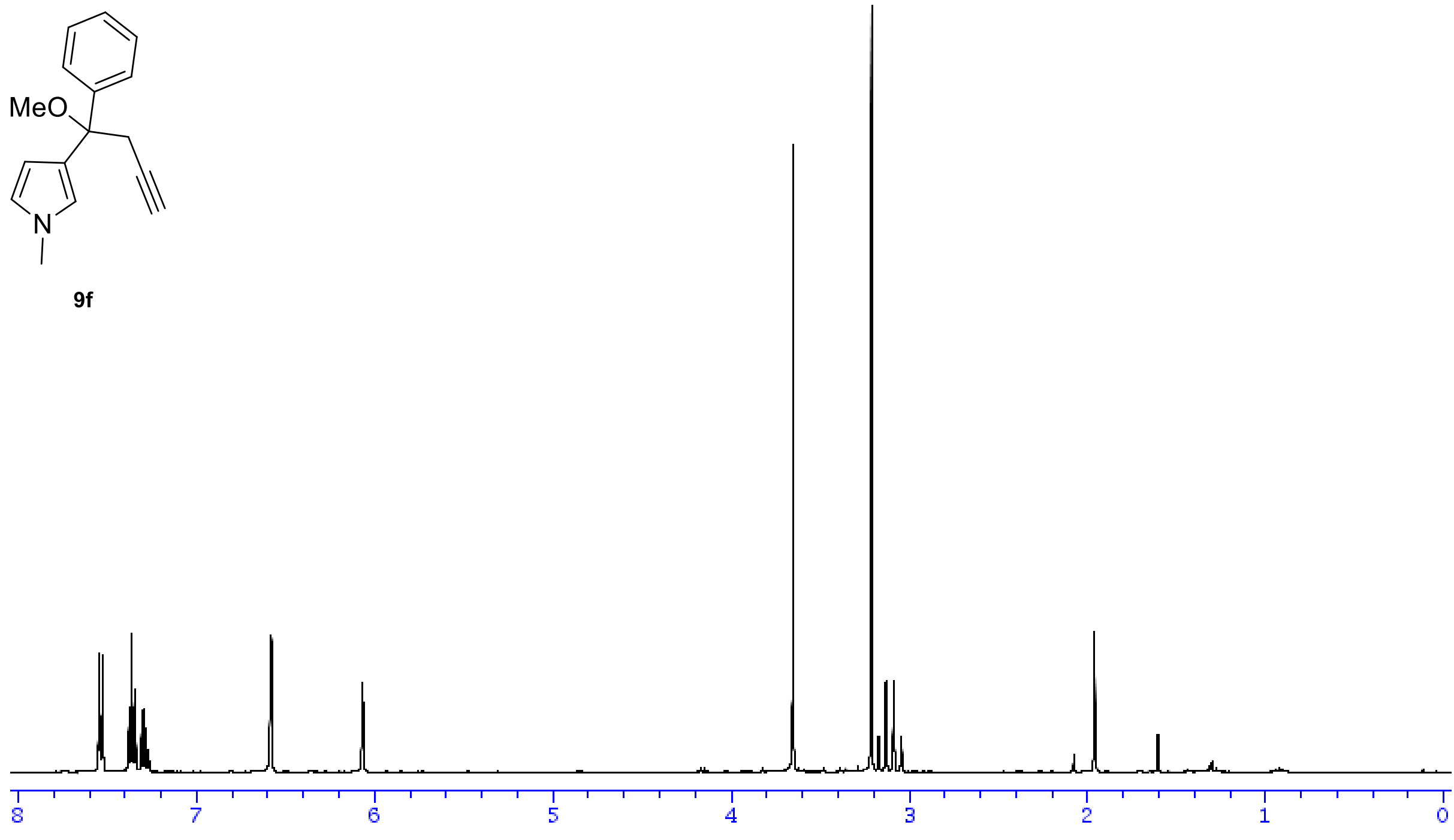

S94 
solvent: $\langle\mathrm{CDCl} 3\rangle$

Frequency. $100.612769 \mathrm{MHz}$

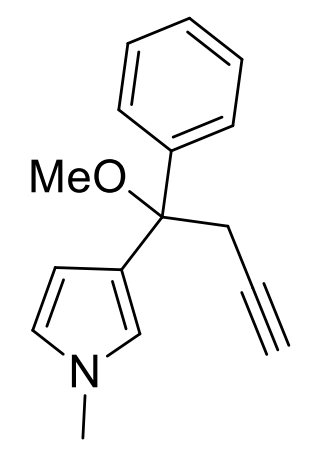

9f

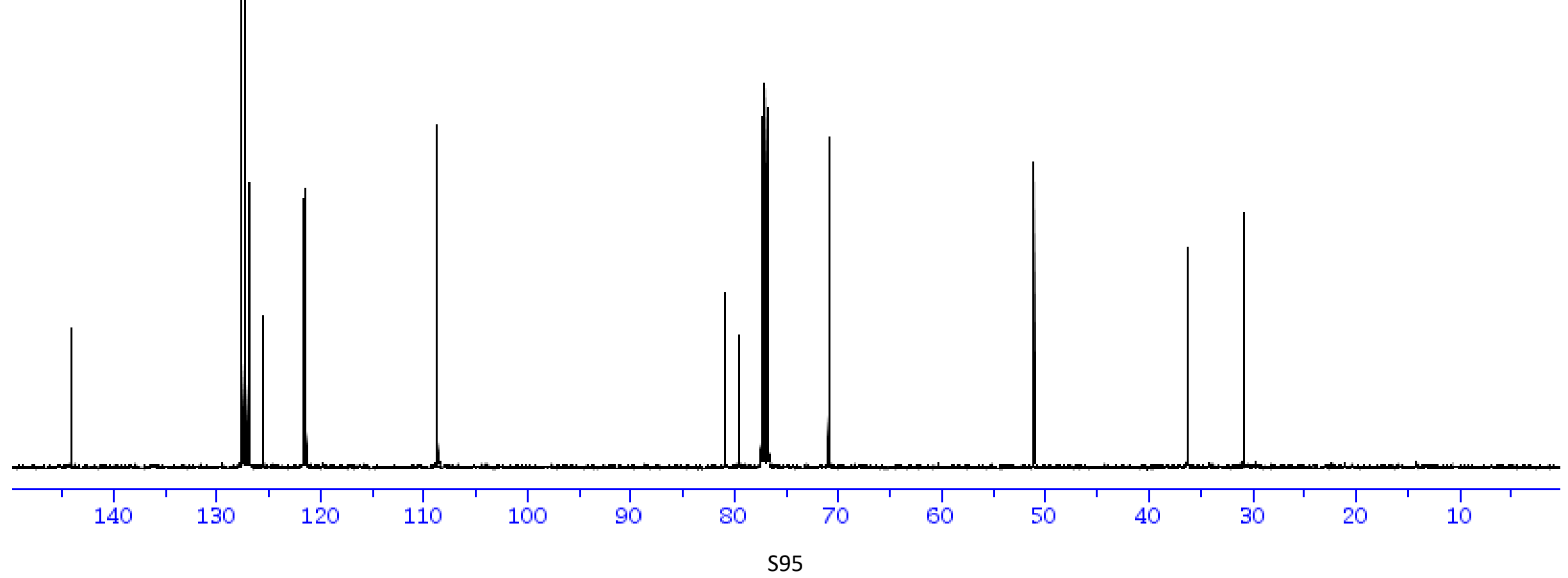



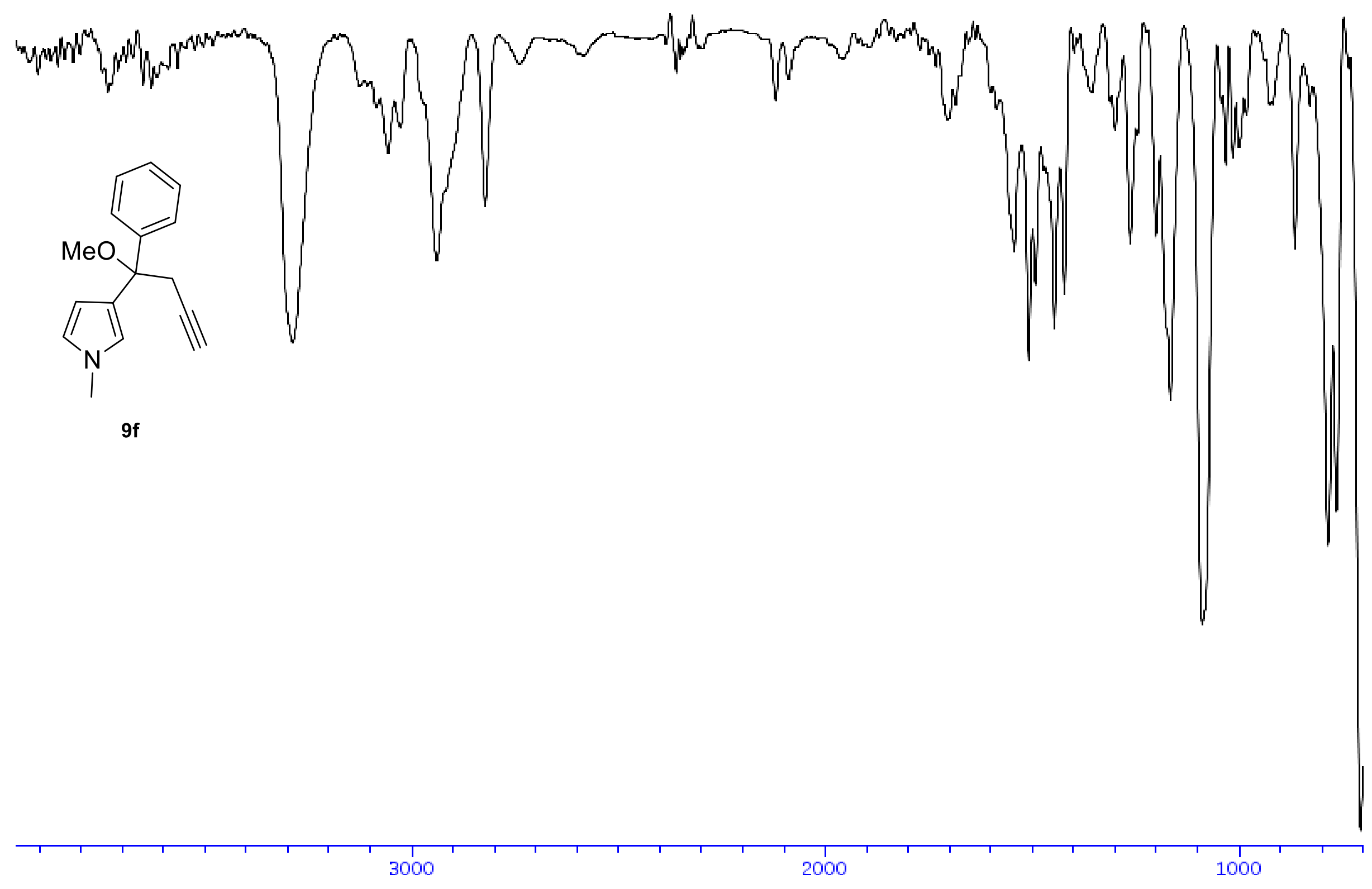
solvent: $\angle \mathrm{CDCl} 3\rangle$

Frequency. $400.13 \mathrm{MHz}$

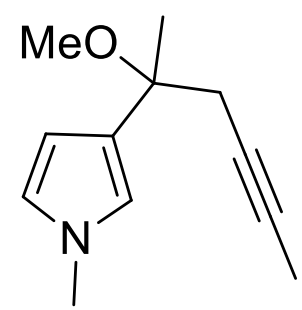

$9 g$

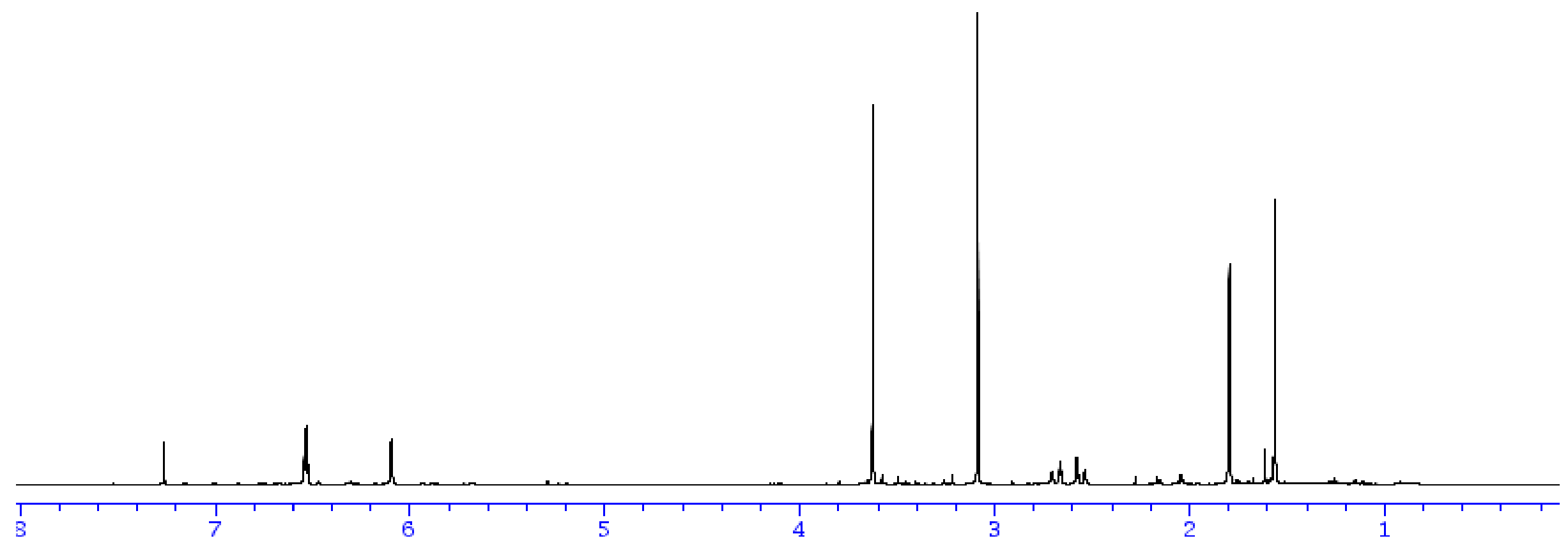


solvent: $\langle\mathrm{CDCI} 3\rangle$

Frequency. $100.612769 \mathrm{MHz}$

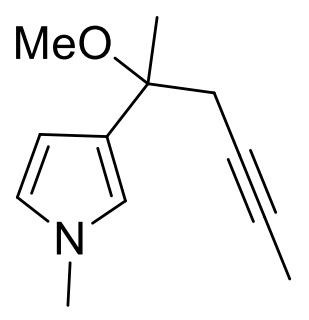

$9 g$
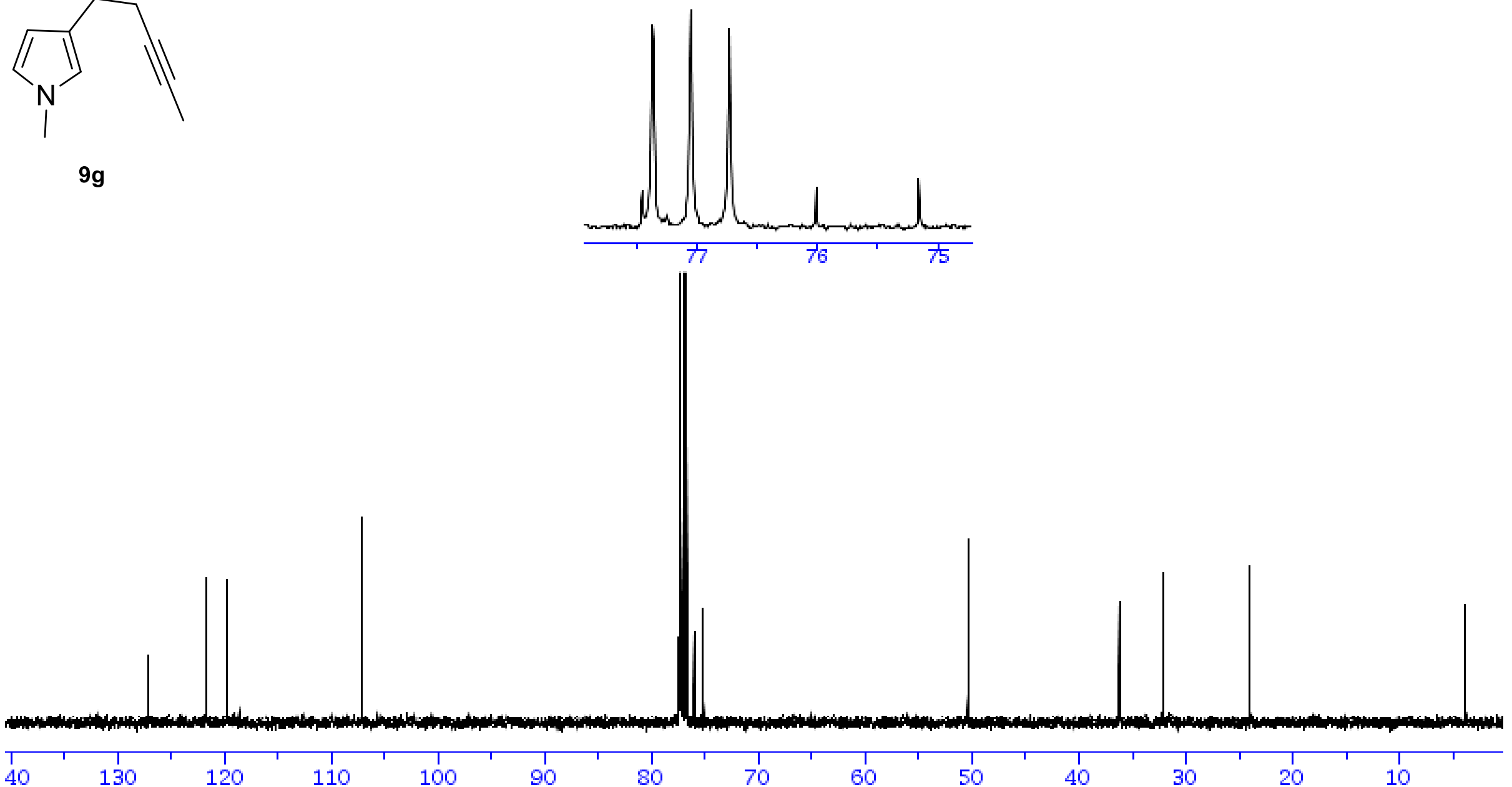


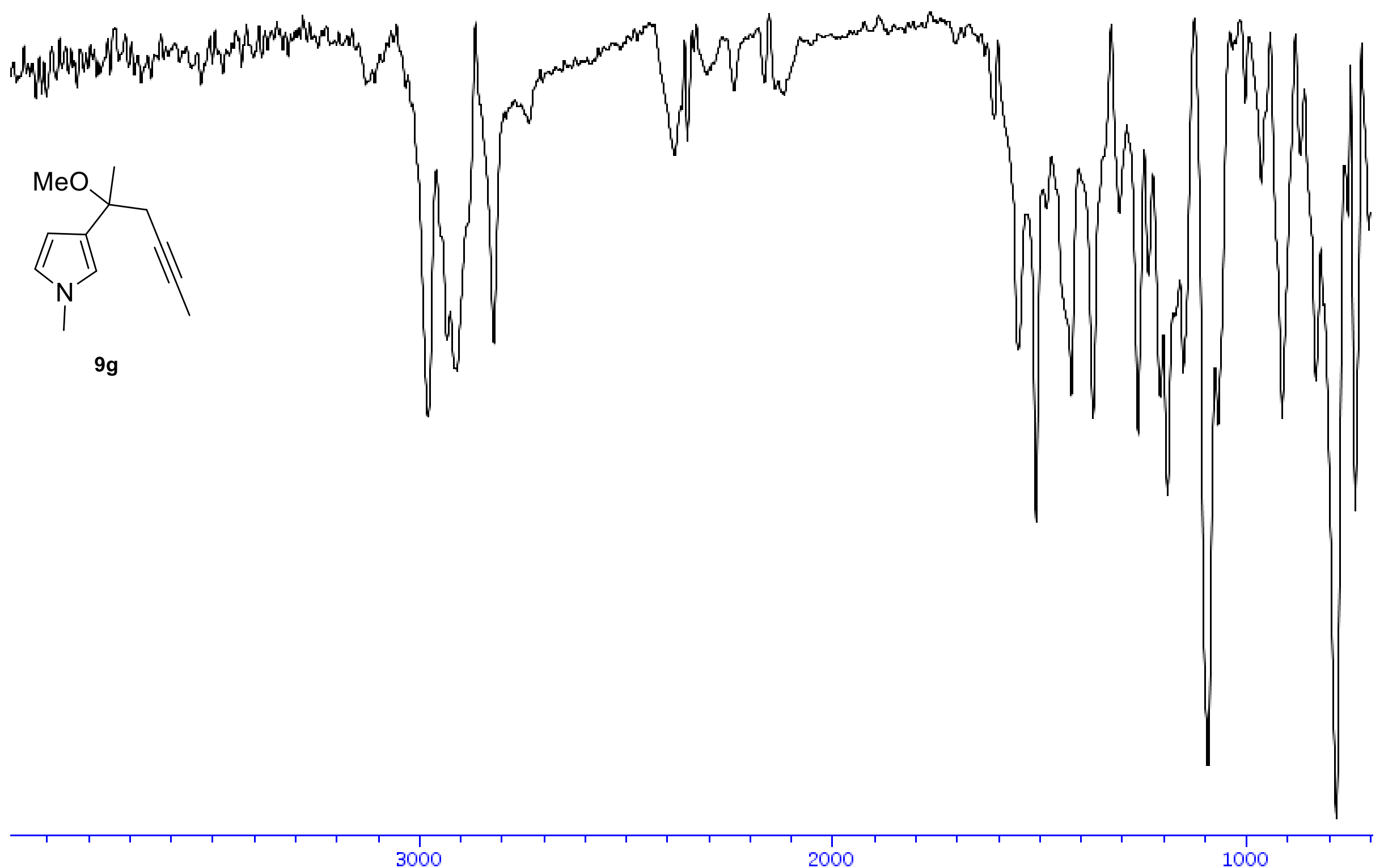

S99 
solvent: $<\mathrm{CDCl} 3>$

Frequency. $400.13 \mathrm{MHz}$

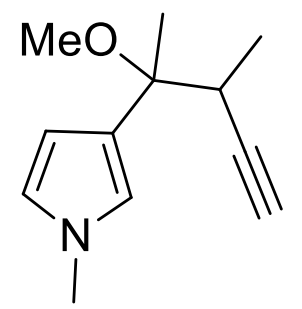

$9 h$
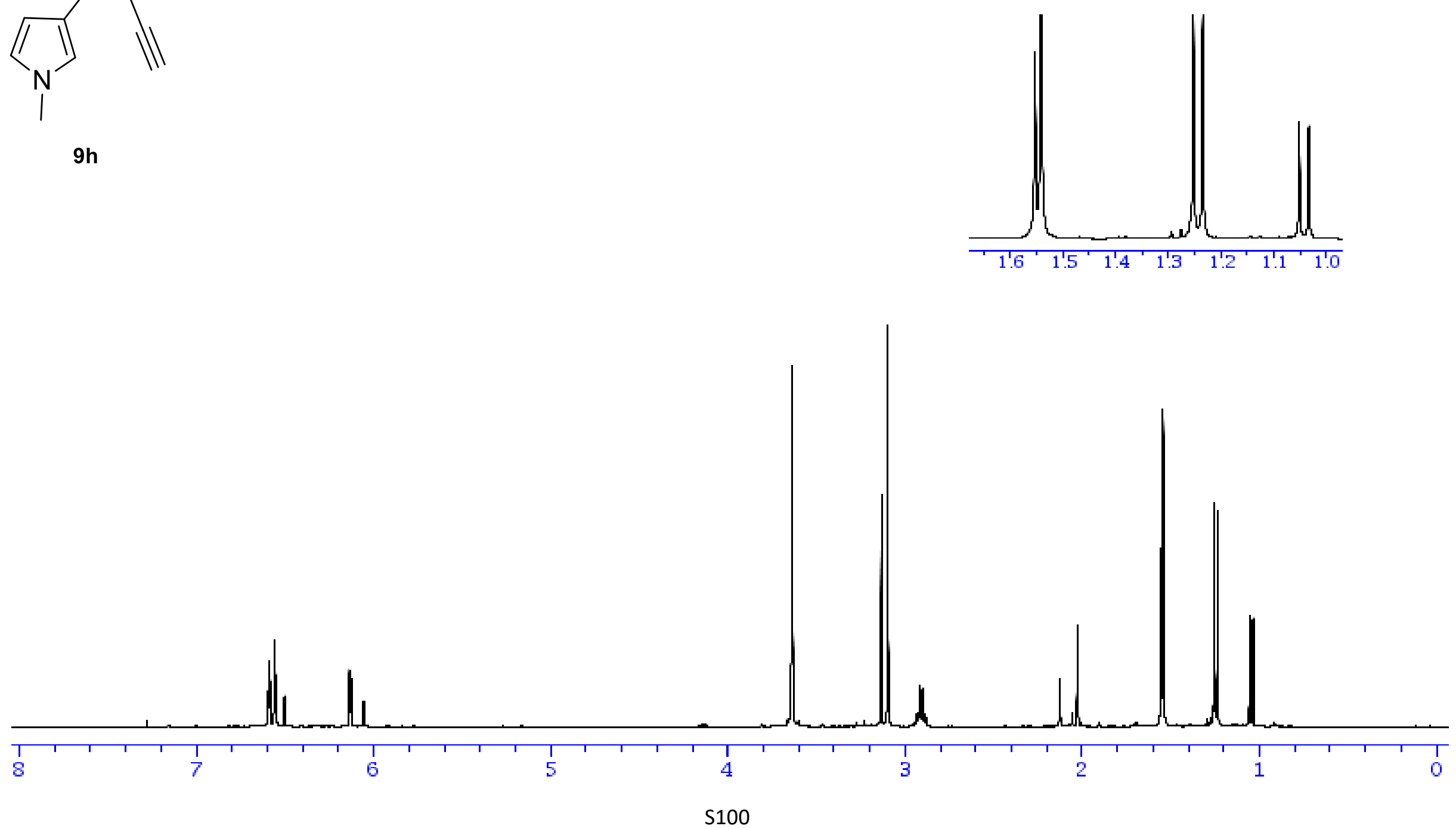
solvent: $\langle\mathrm{CDCl} 3>$

Frequency. 100.612769M Hz

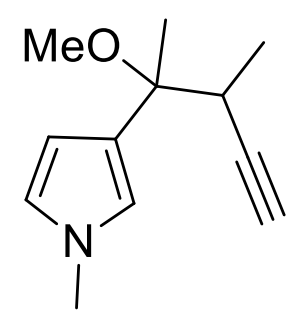

$9 h$
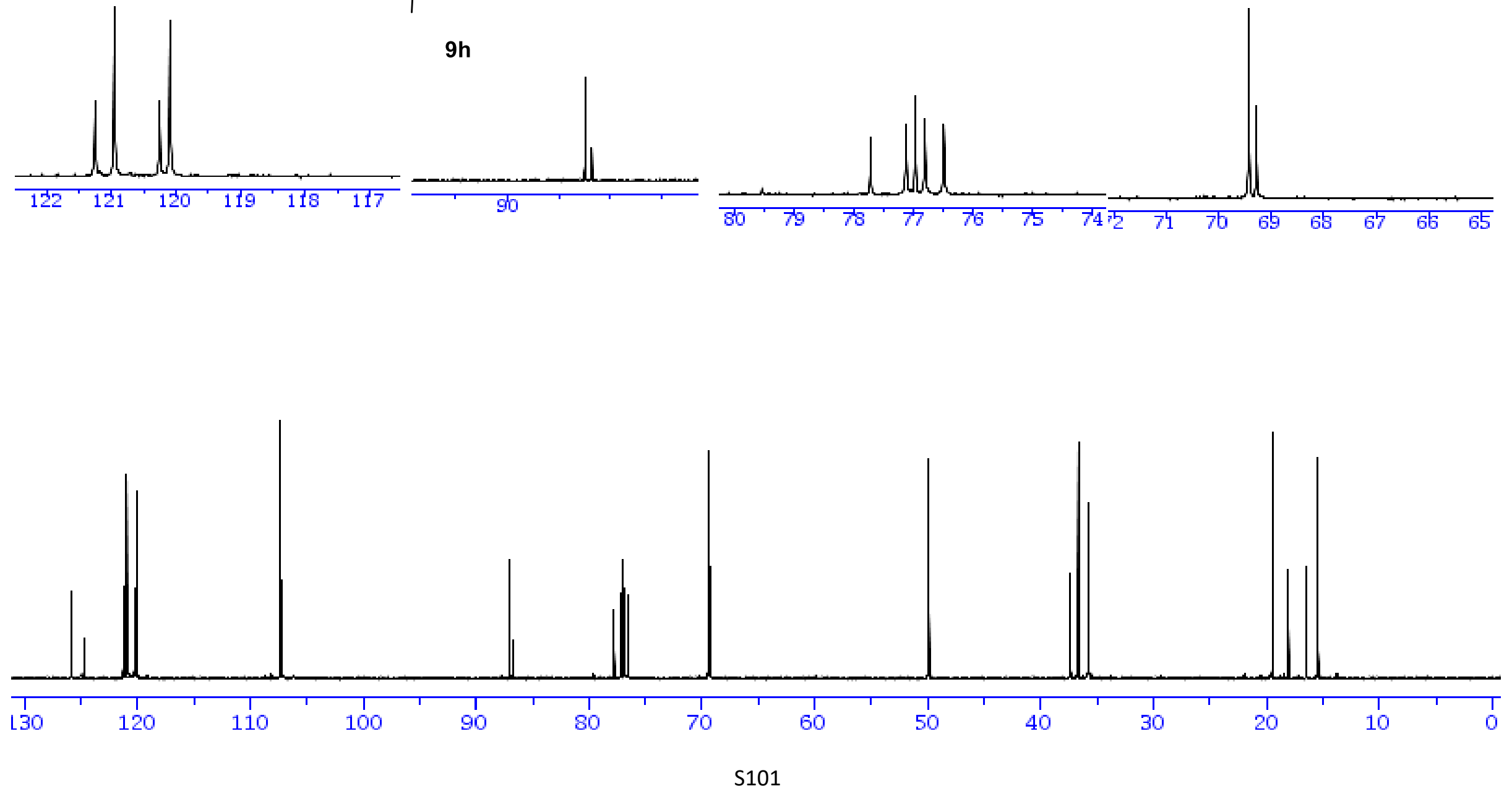


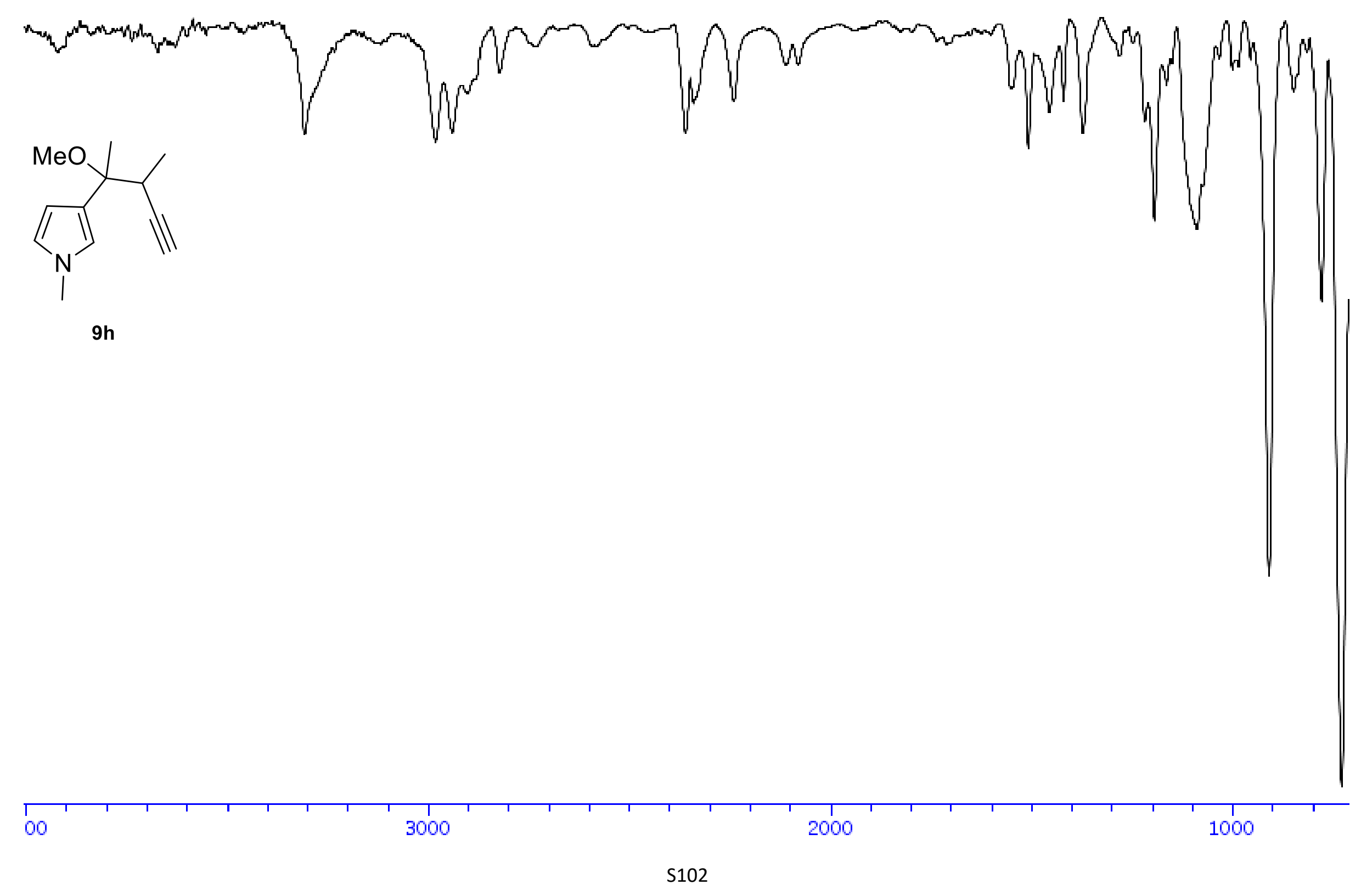


solvent: $<\mathrm{CDCI}>$

Frequency. $400.13 \mathrm{MHz}$
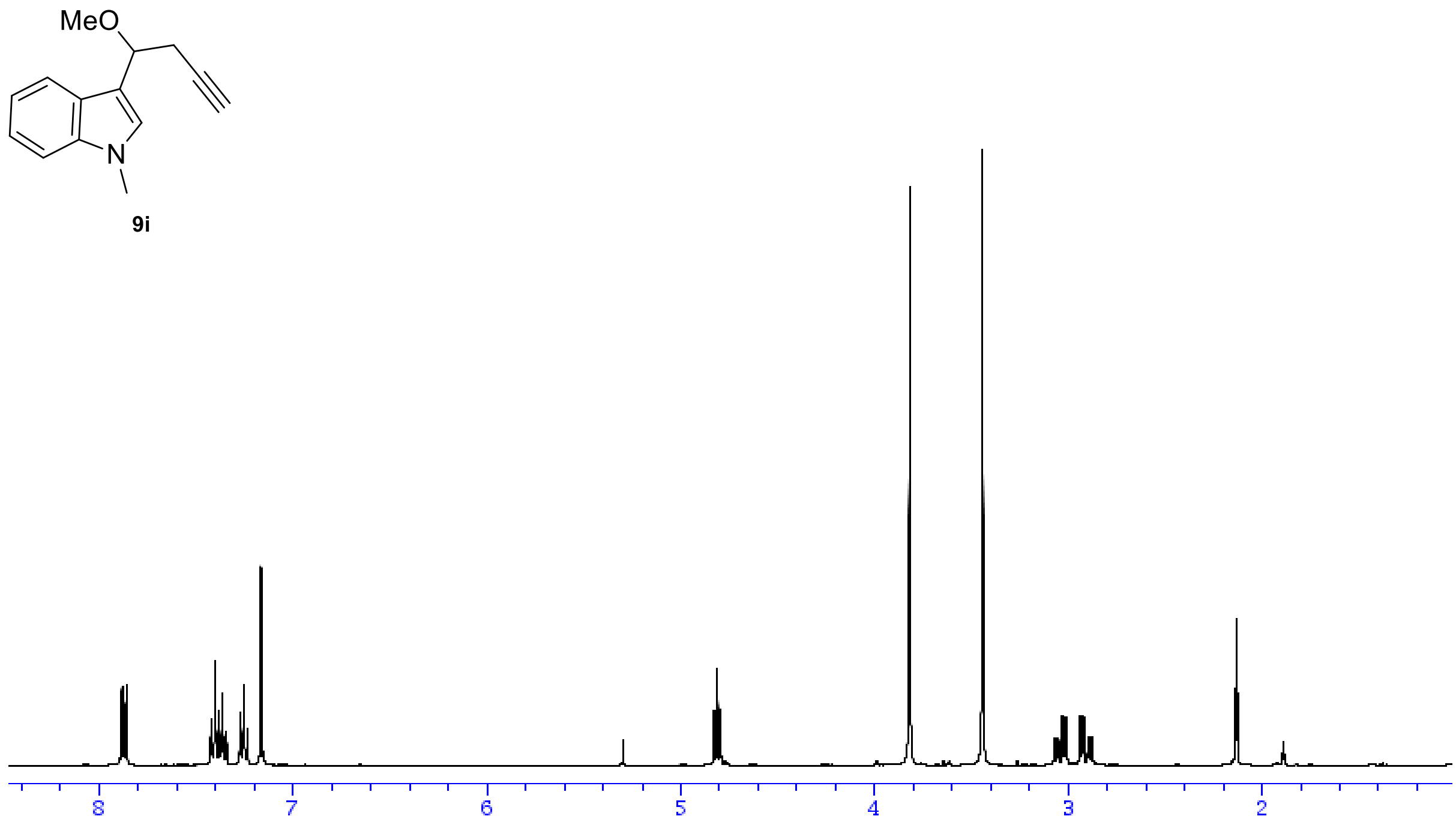

S103 
solvent: $\langle\mathrm{CDCl} 3>$

Frequency. $100.612769 \mathrm{MHz}$
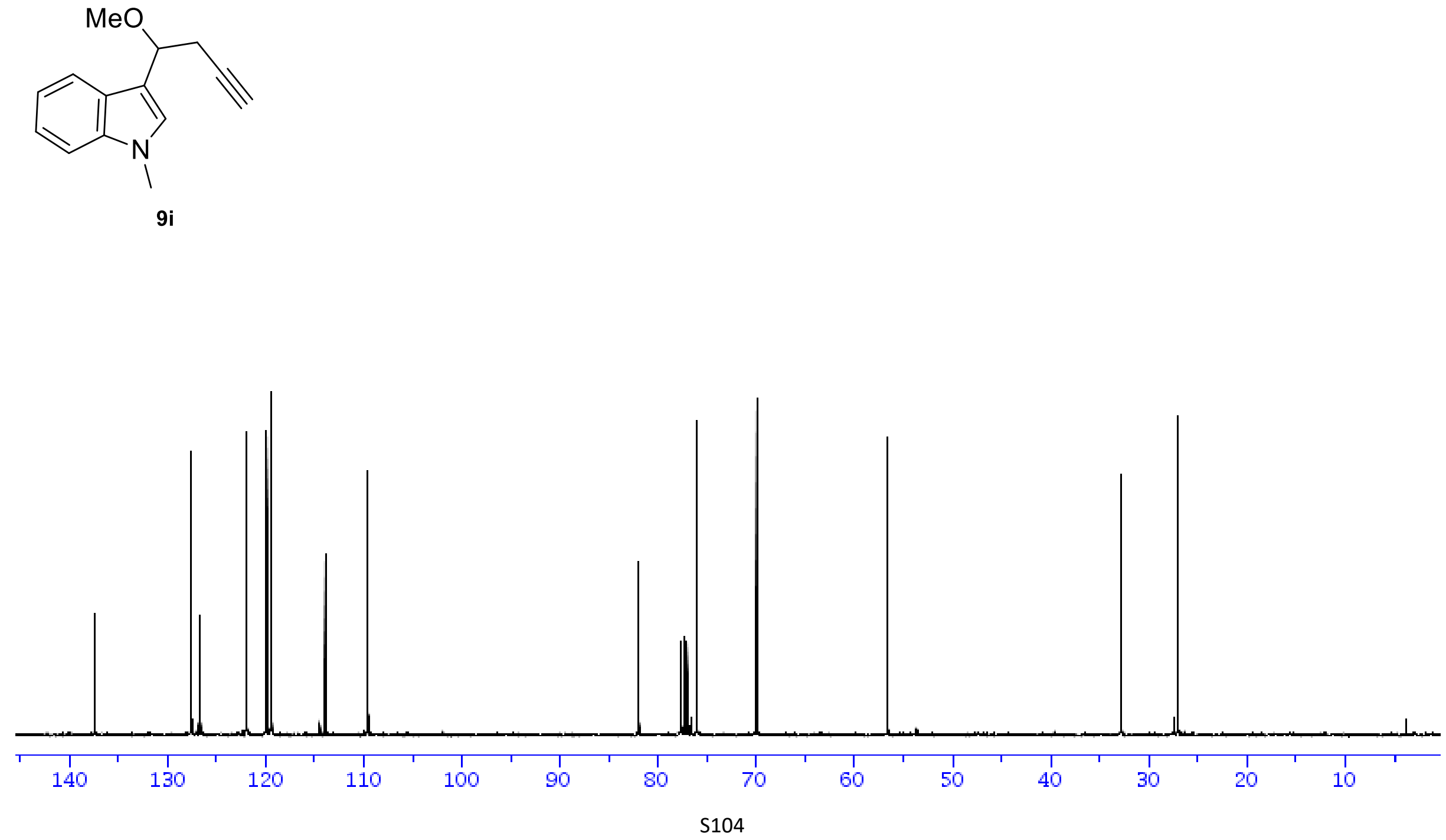


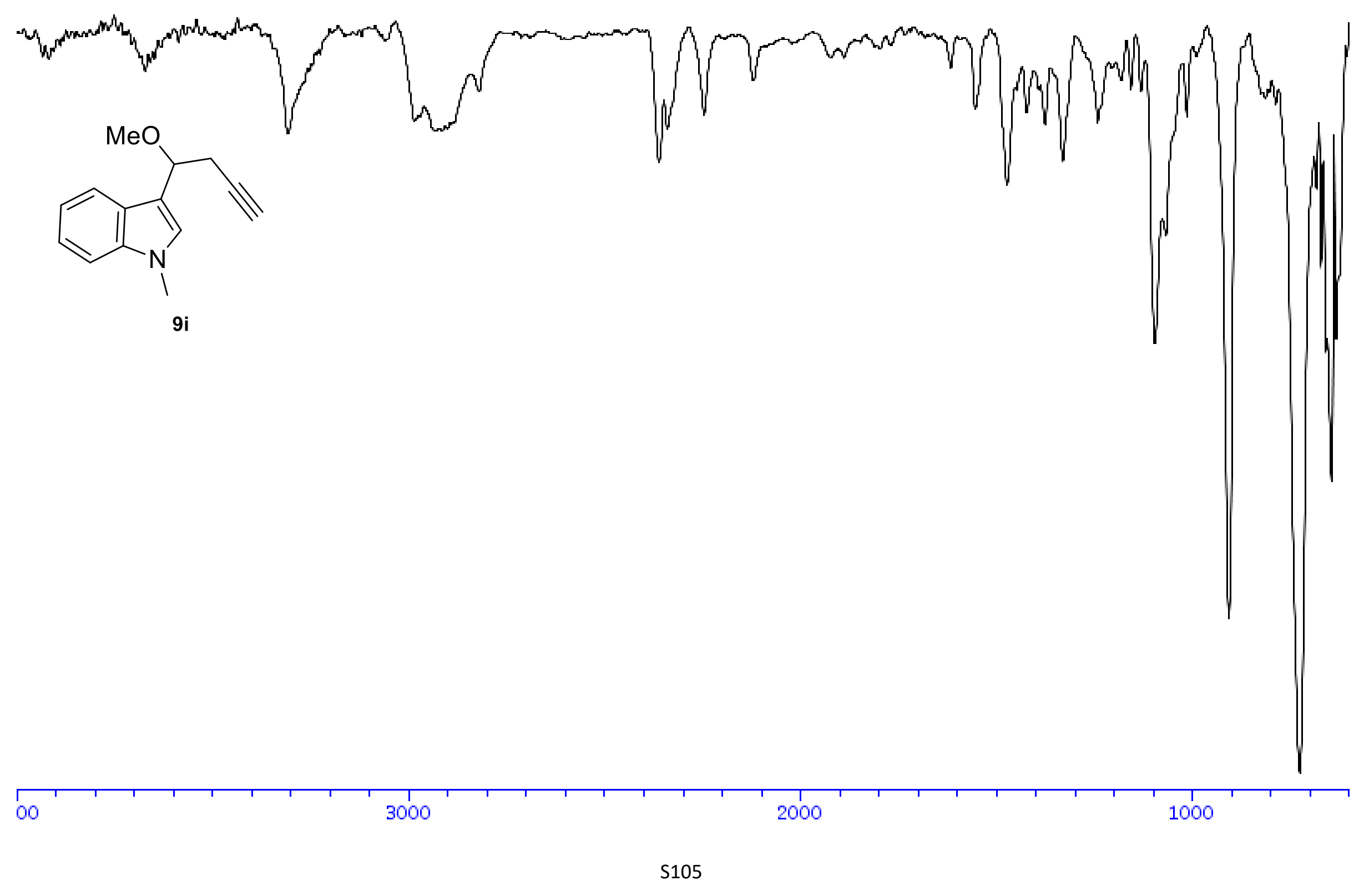


solvent: $<C D C I 3>$

Frequency. $400.13 \mathrm{MHz}$

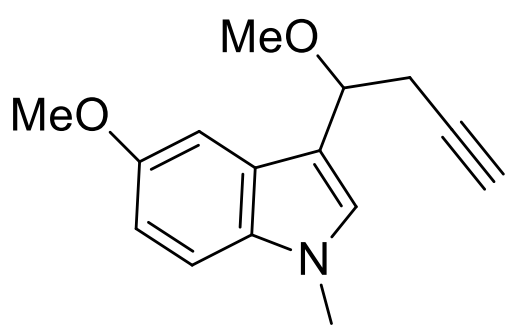

jj

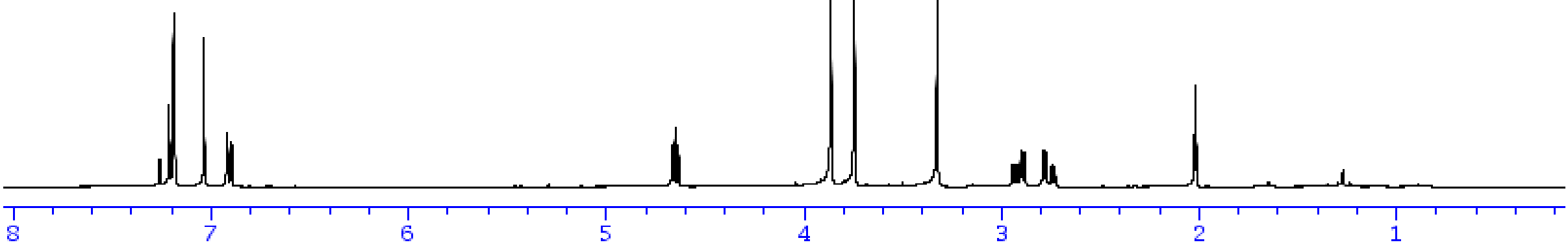

S106 
solvent: $\langle\mathrm{CDCI}>$

Frequency. $100.612769 \mathrm{MHz}$
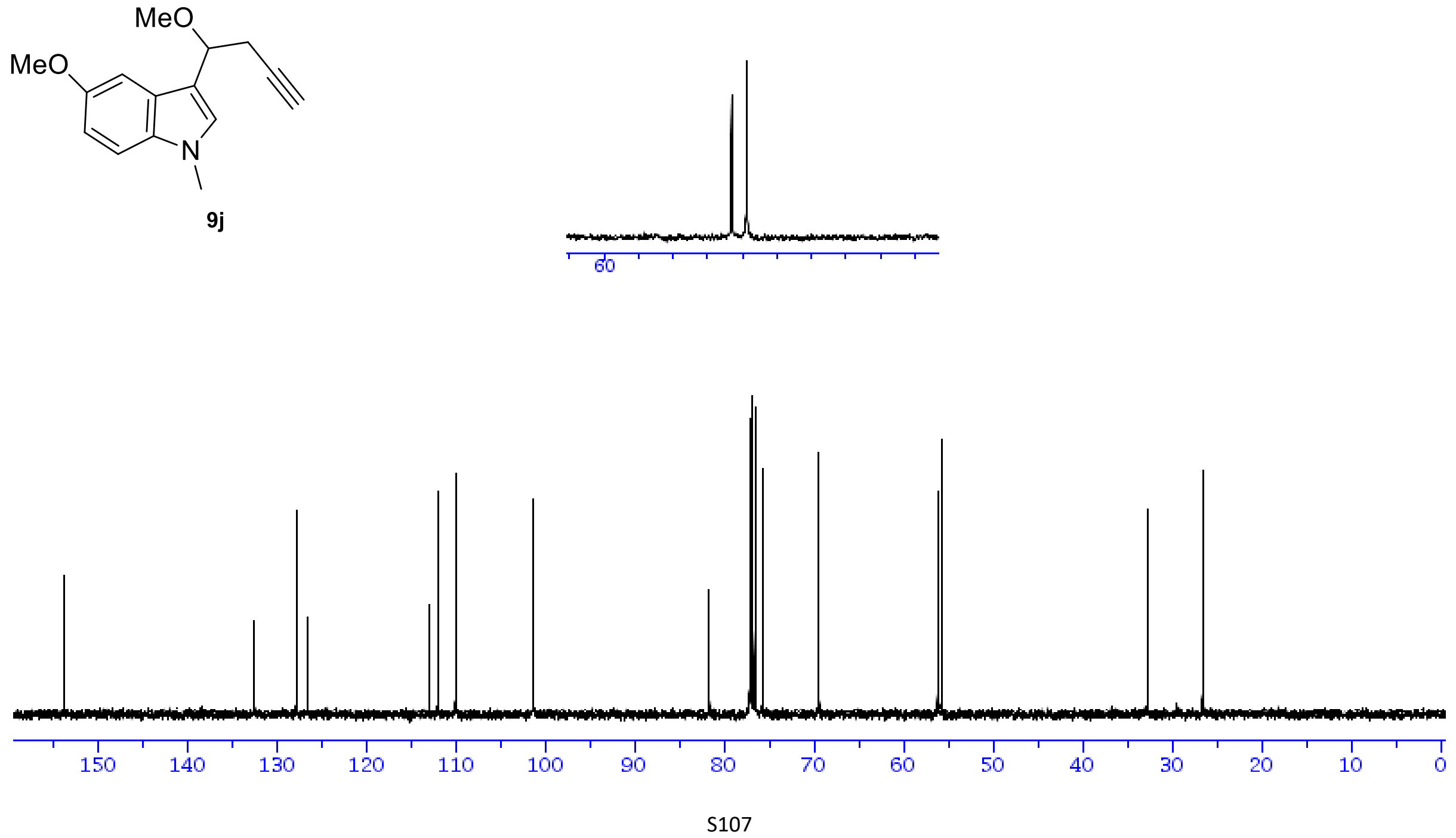


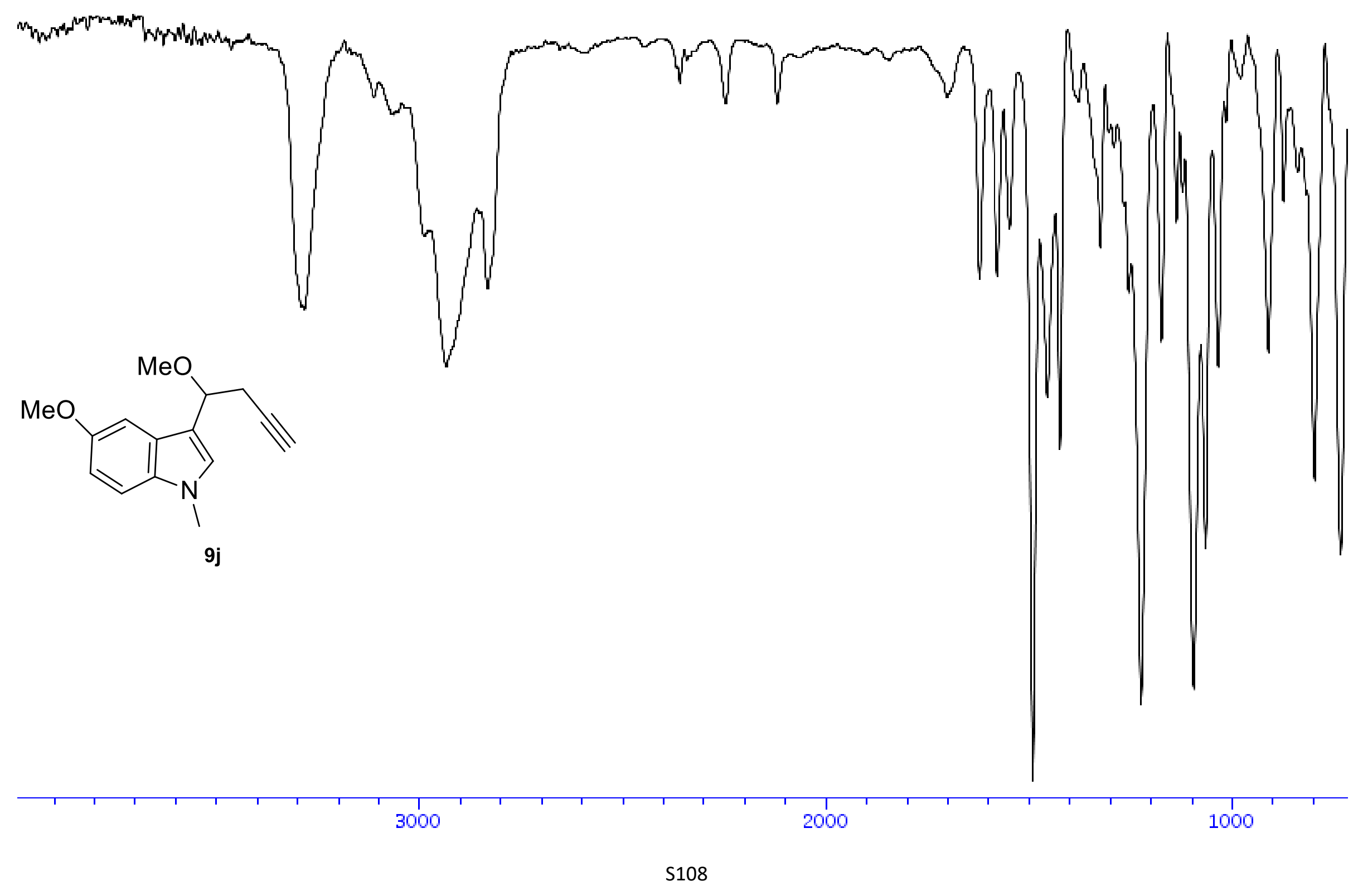


solvent: $\langle\mathrm{CDCl} 3\rangle$

Frequency. $400.13 \mathrm{MHz}$

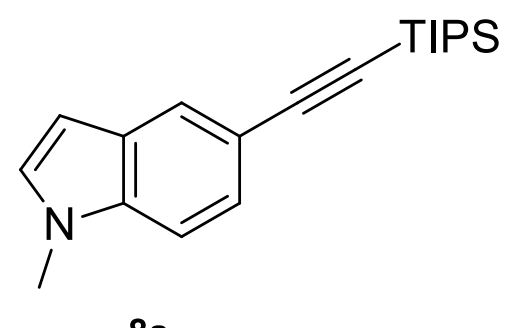

$8 a$

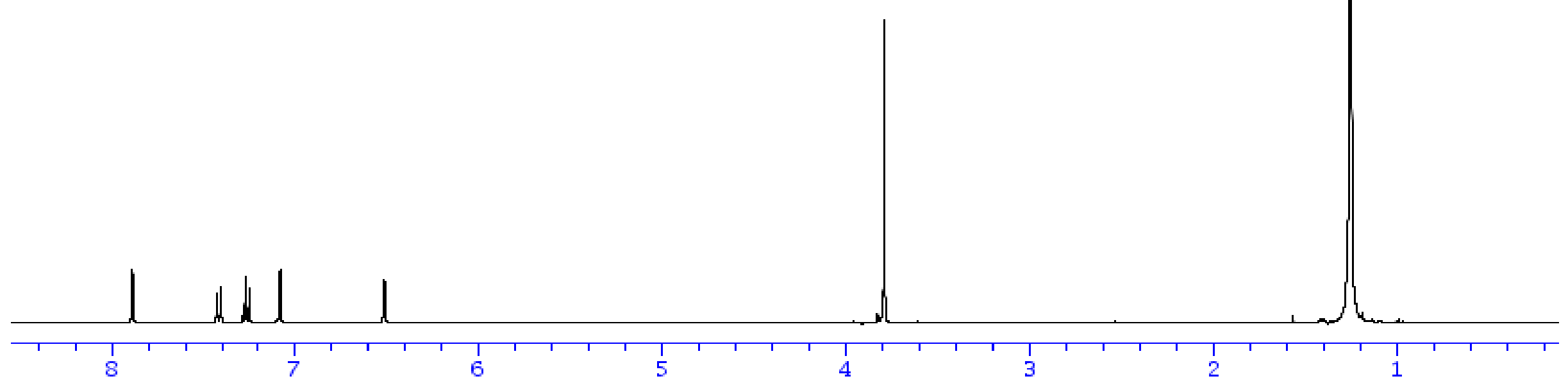


solvent: $\langle\mathrm{CDCl} 3\rangle$

Frequency. 100.612769M Hz

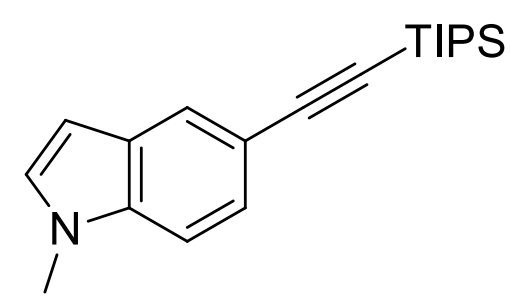

$8 a$
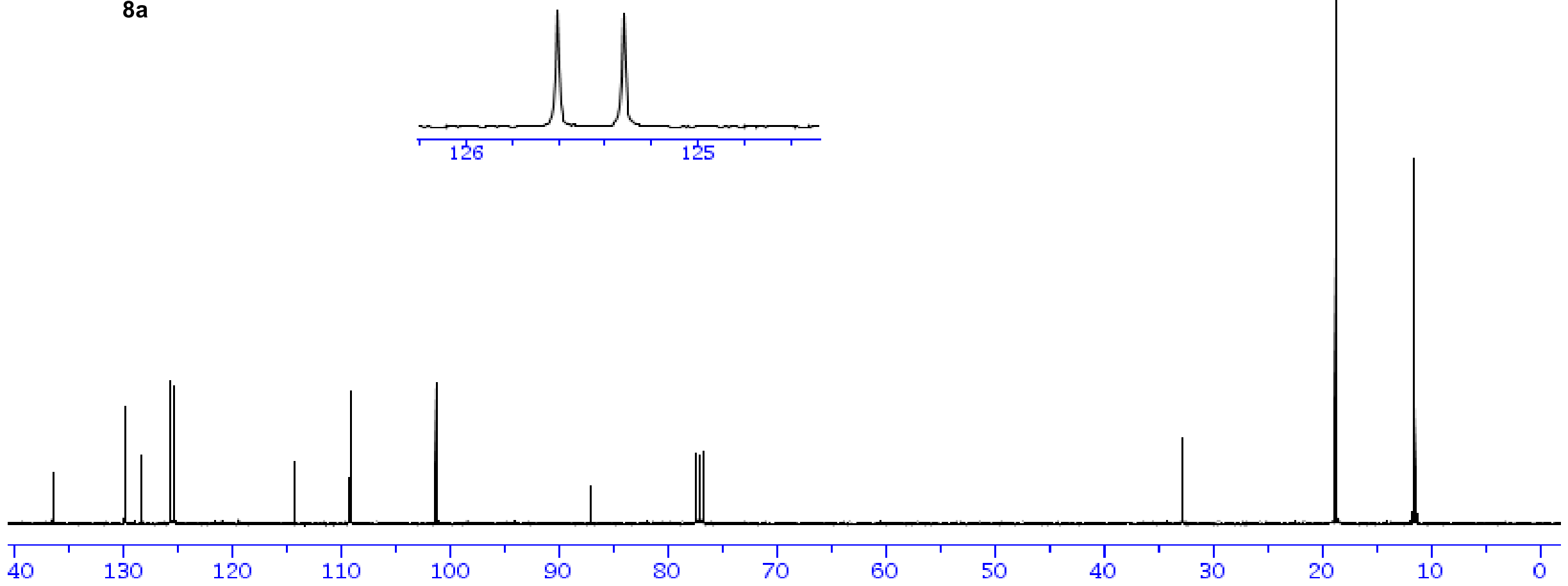


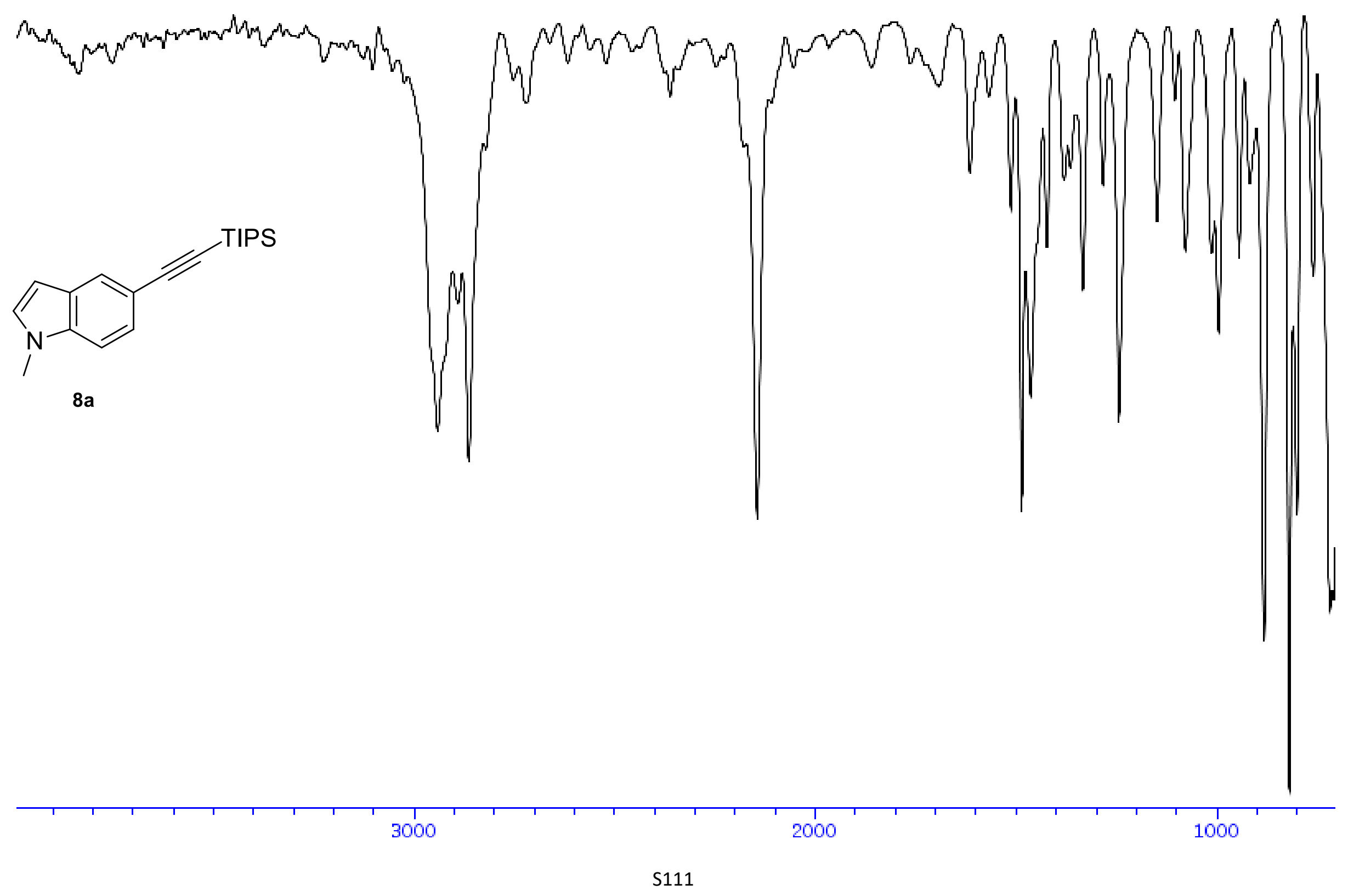


solvent: $\langle\mathrm{CDCl} 3>$

Frequency. $400.13 \mathrm{MHz}$

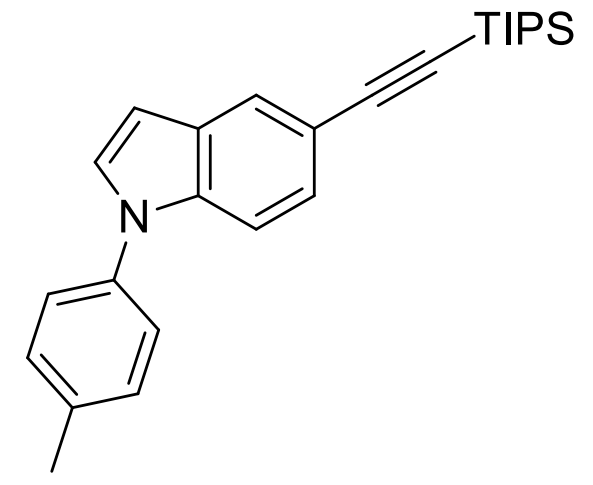

8b
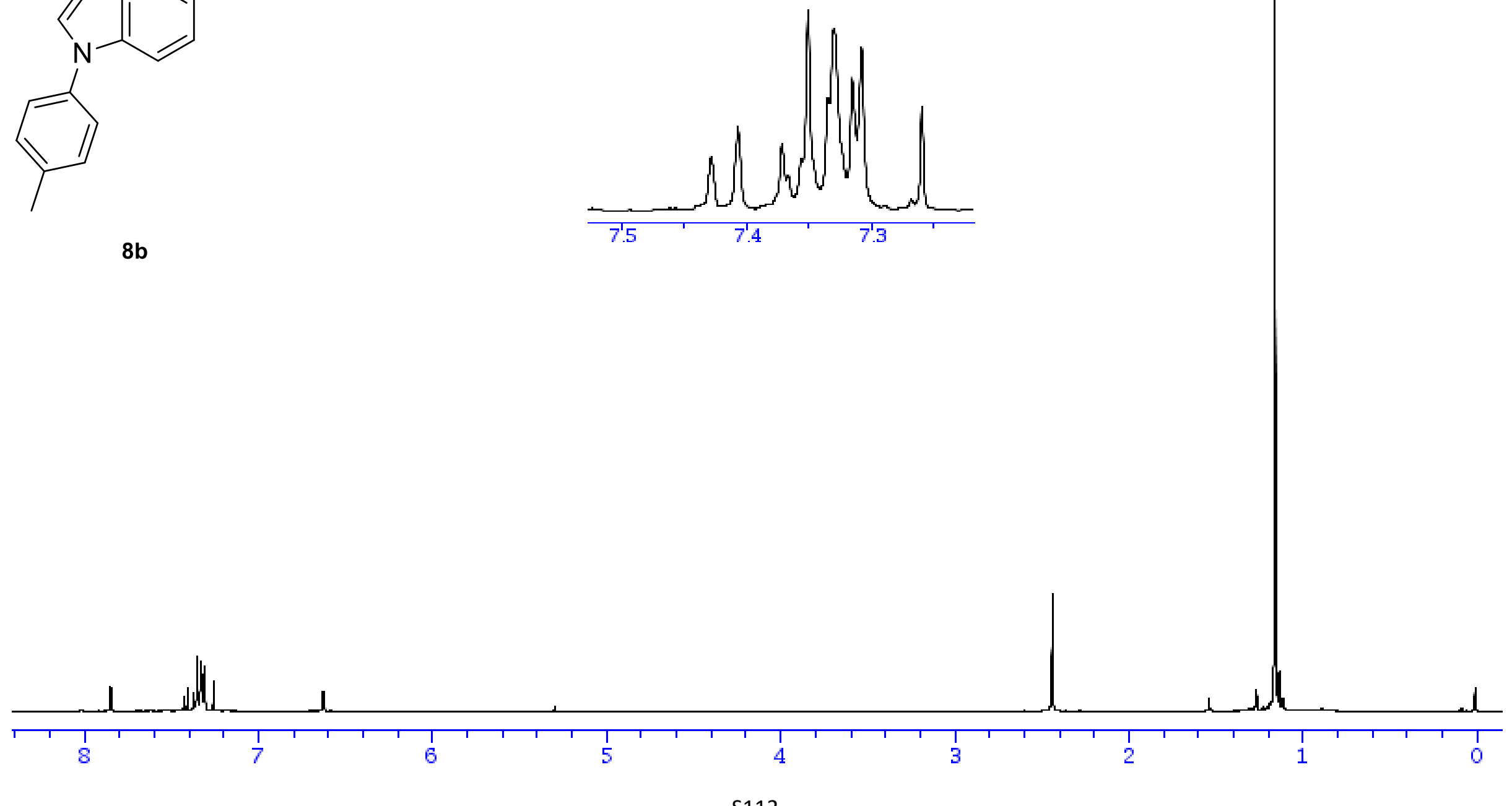
solvent: $\langle\mathrm{CDCI}>$

Frequency. $100.612769 \mathrm{MHz}$

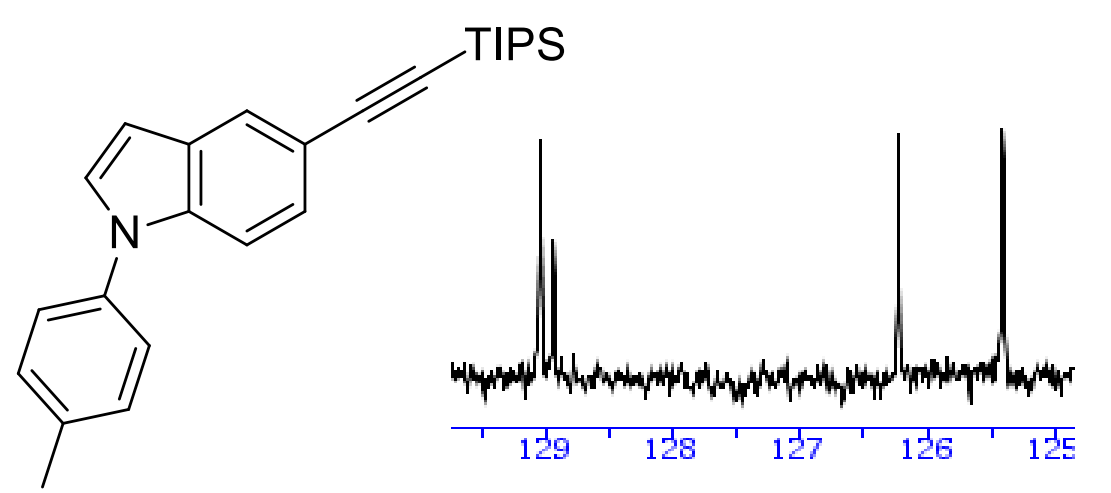

$8 b$

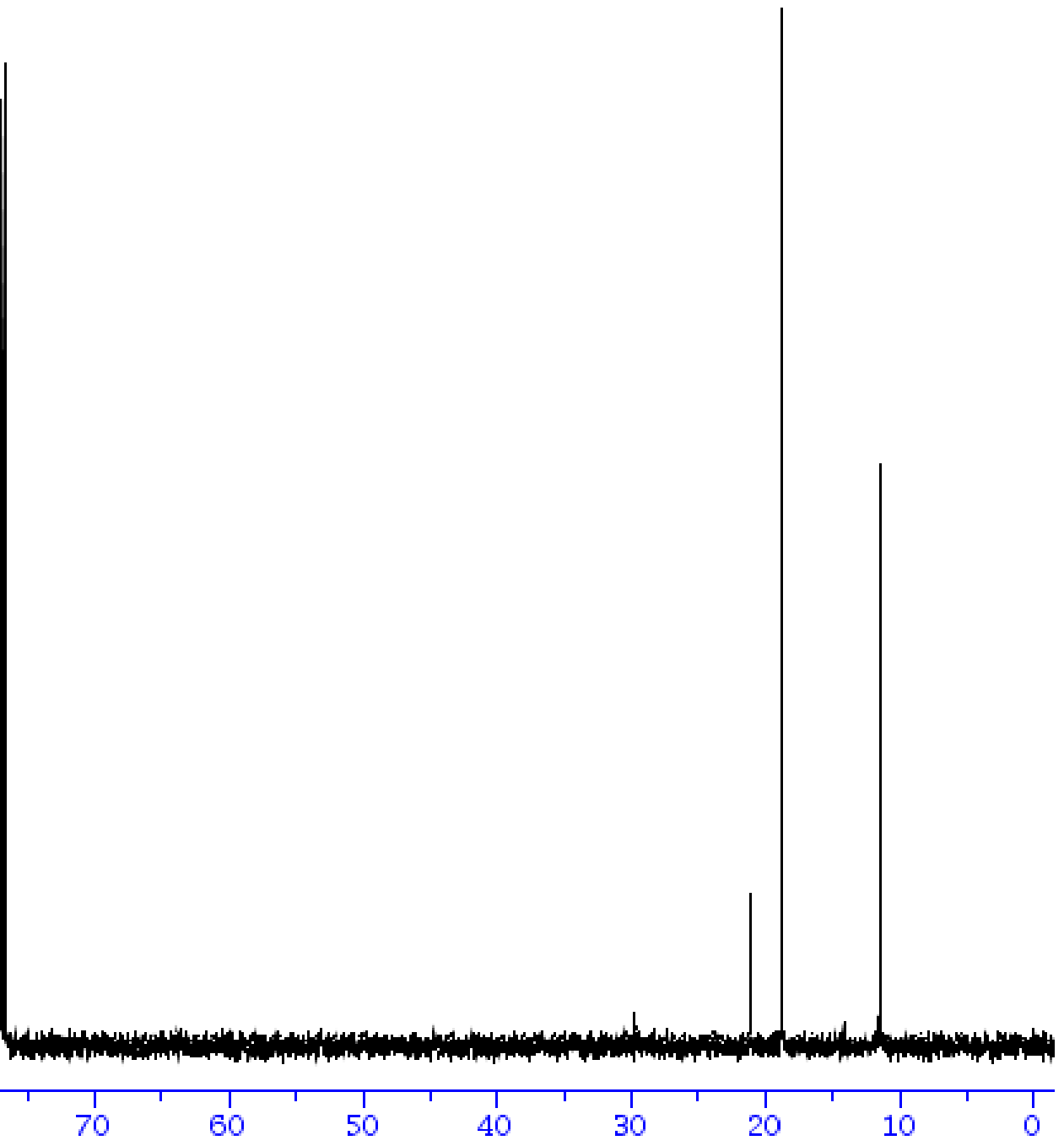




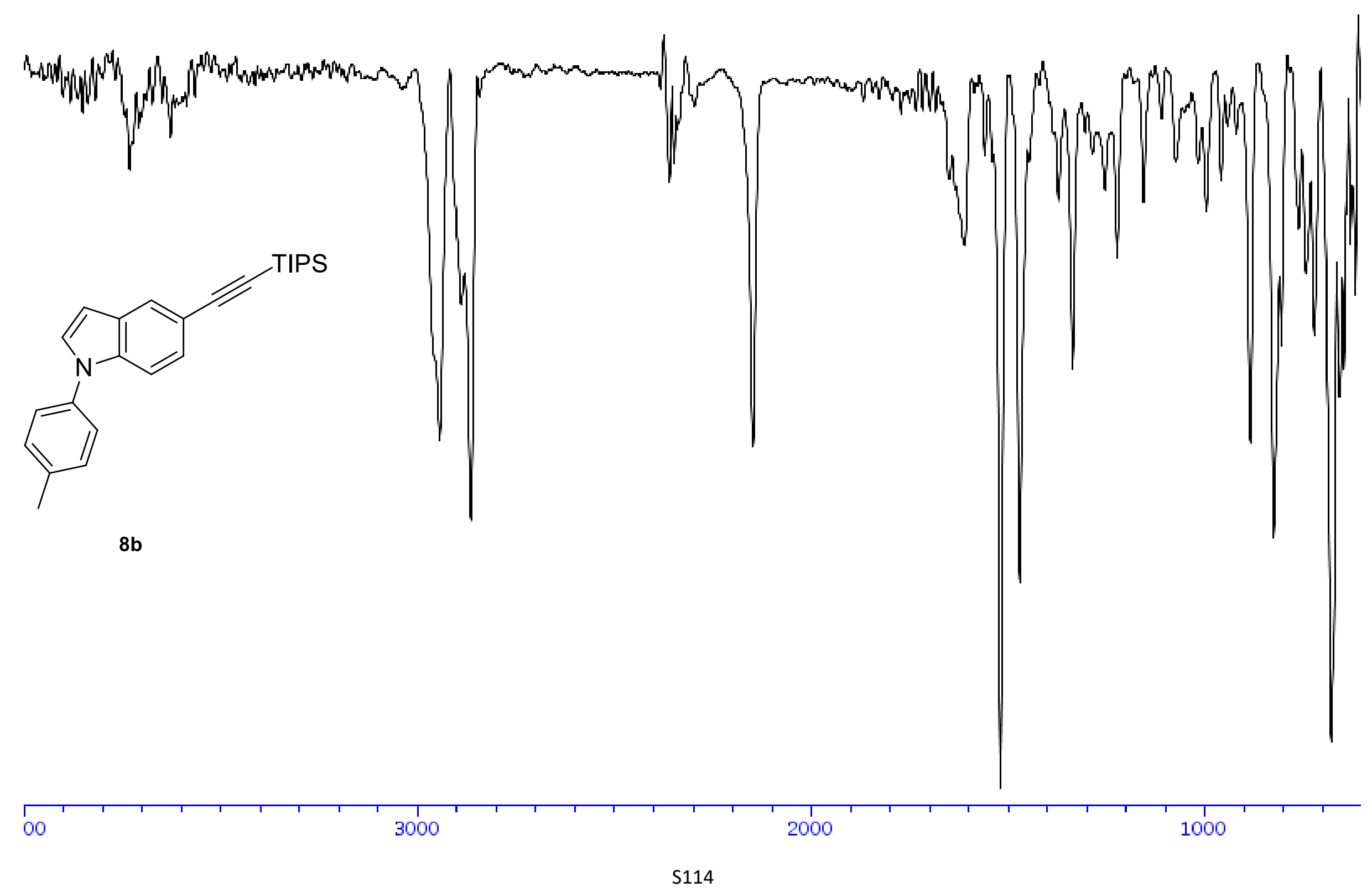


solvent: $\langle\mathrm{CDCl} 3>$

Frequency. $400.13 \mathrm{MHz}$

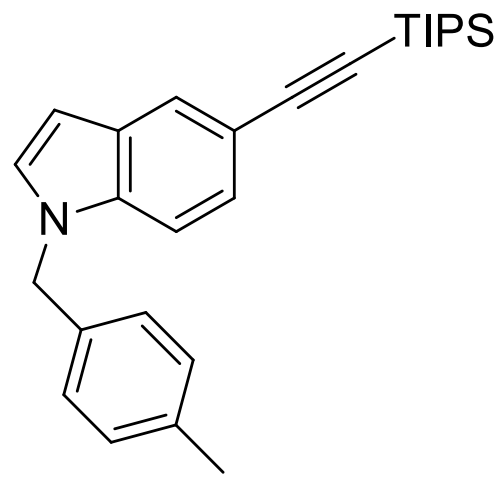

$8 c$
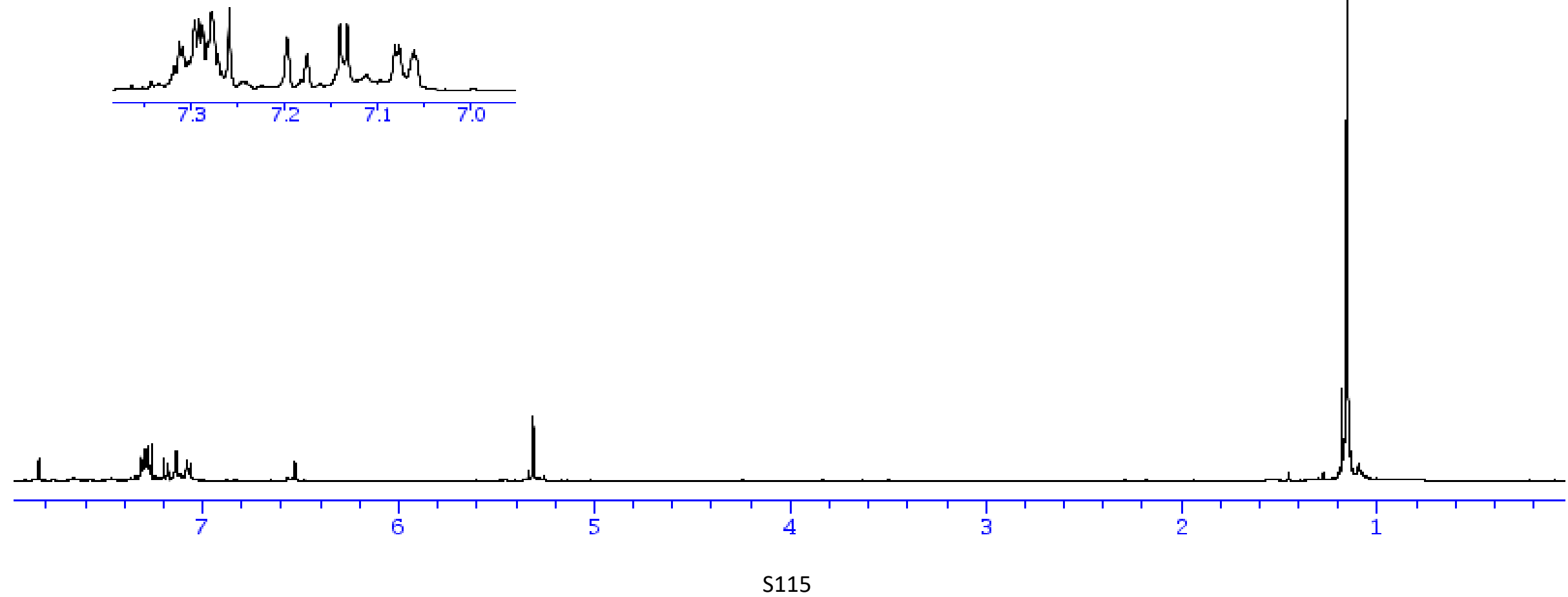
solvent: $\langle\mathrm{CDCl} 3\rangle$

Frequency. $100.612769 \mathrm{MHz}$
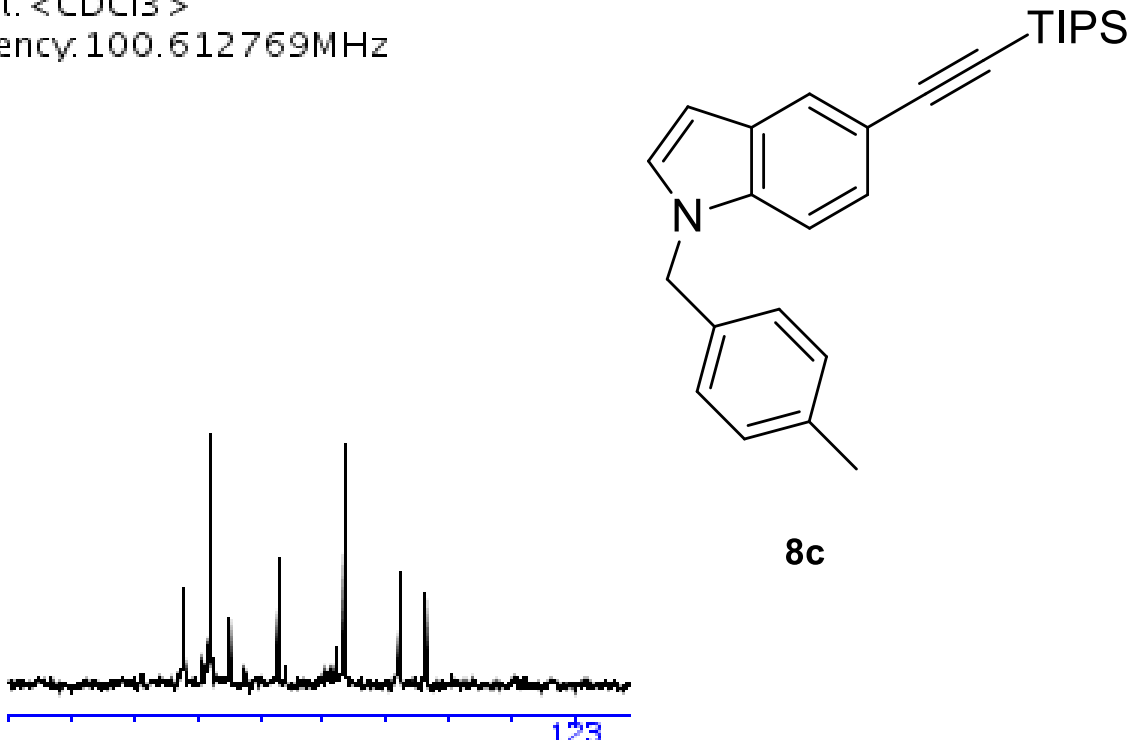

$8 c$
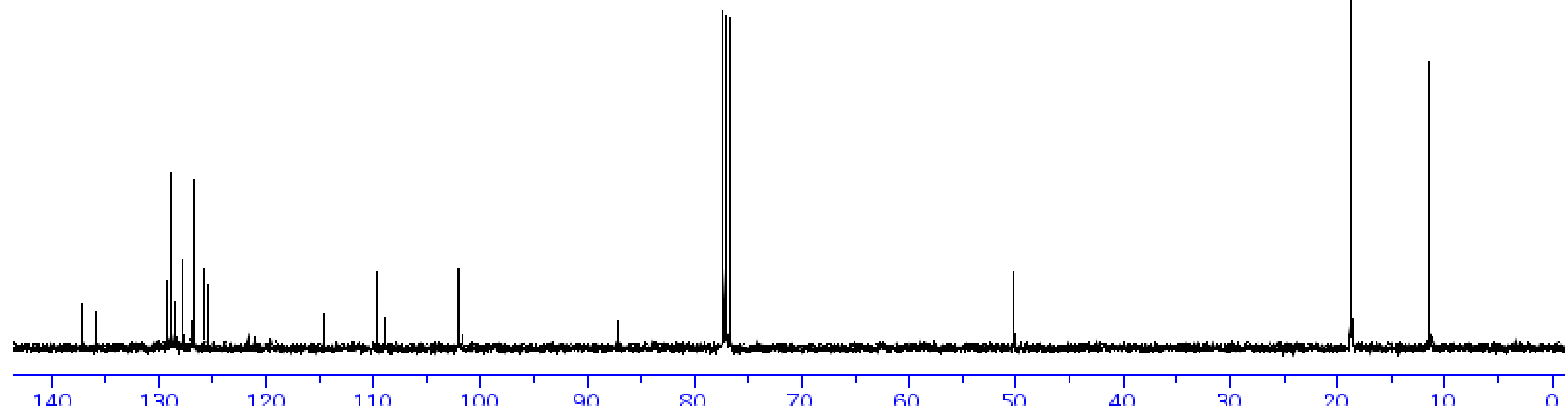


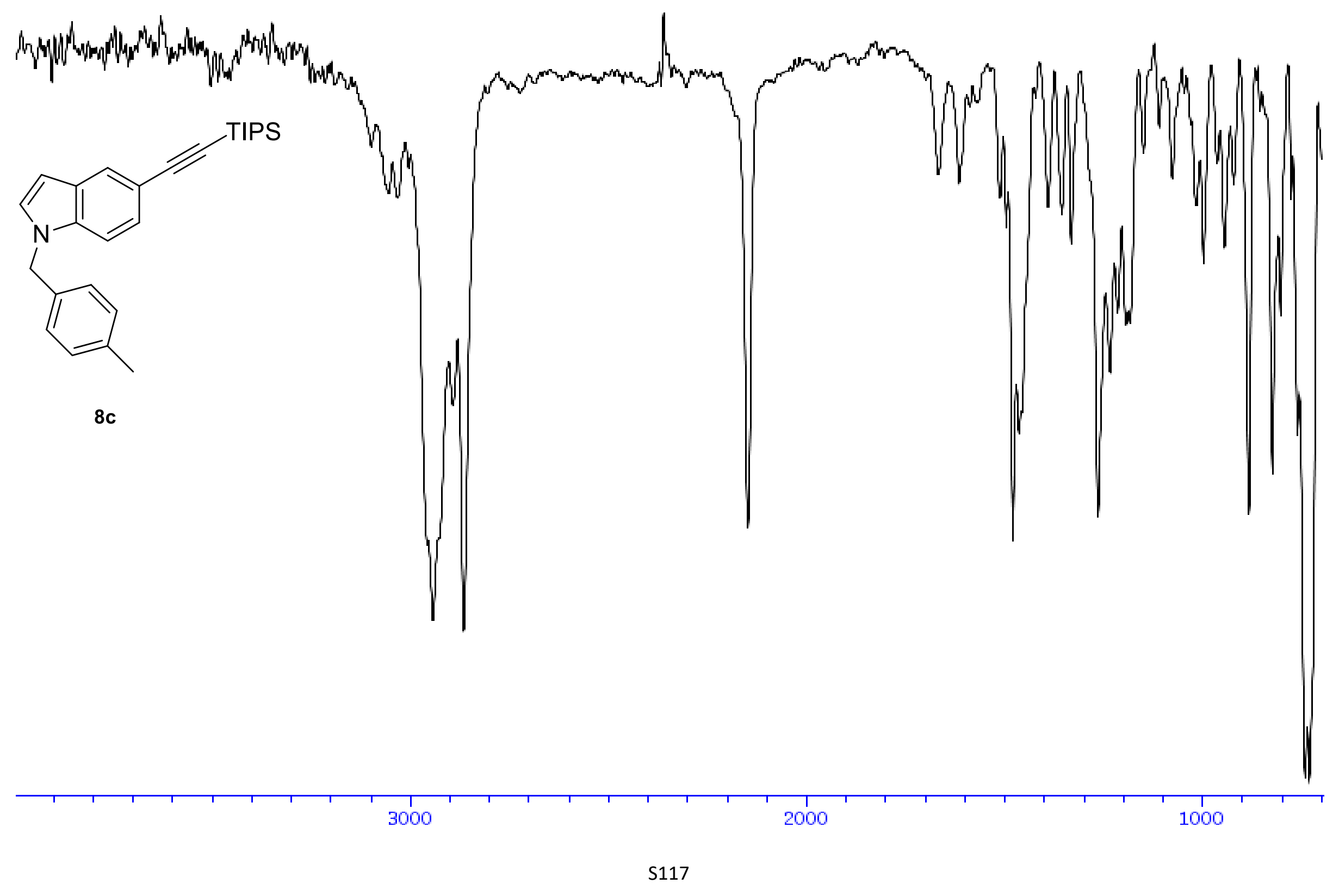


solvent: $\langle\mathrm{CDCl} 3>$

Frequency. 400.13MHz

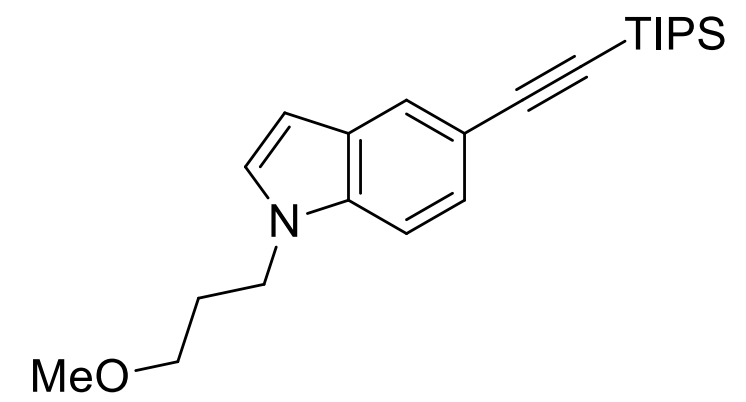

8d

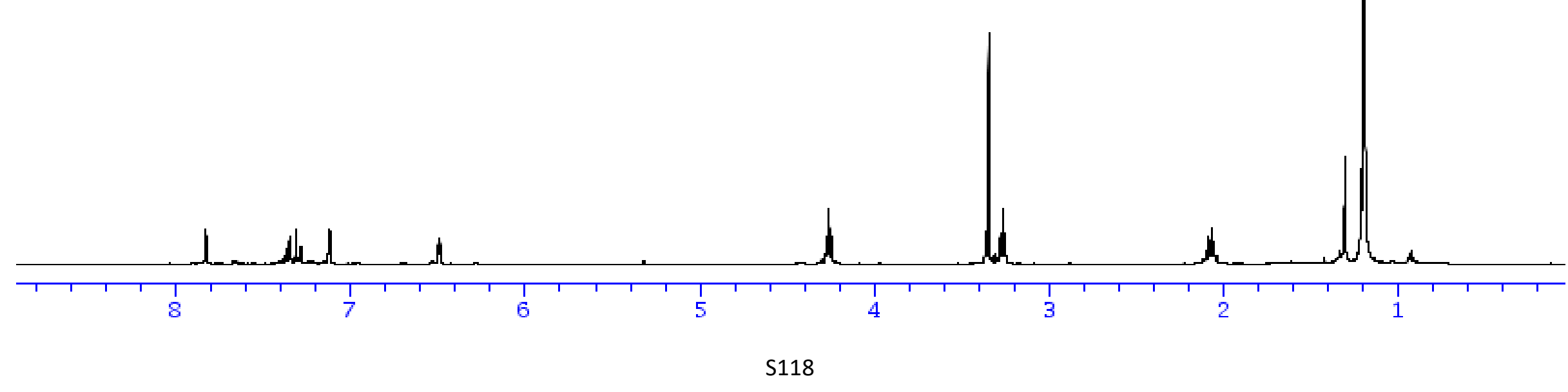


solvent: $\langle\mathrm{CDCl} 3\rangle$

Frequency. $100.612769 \mathrm{MHz}$
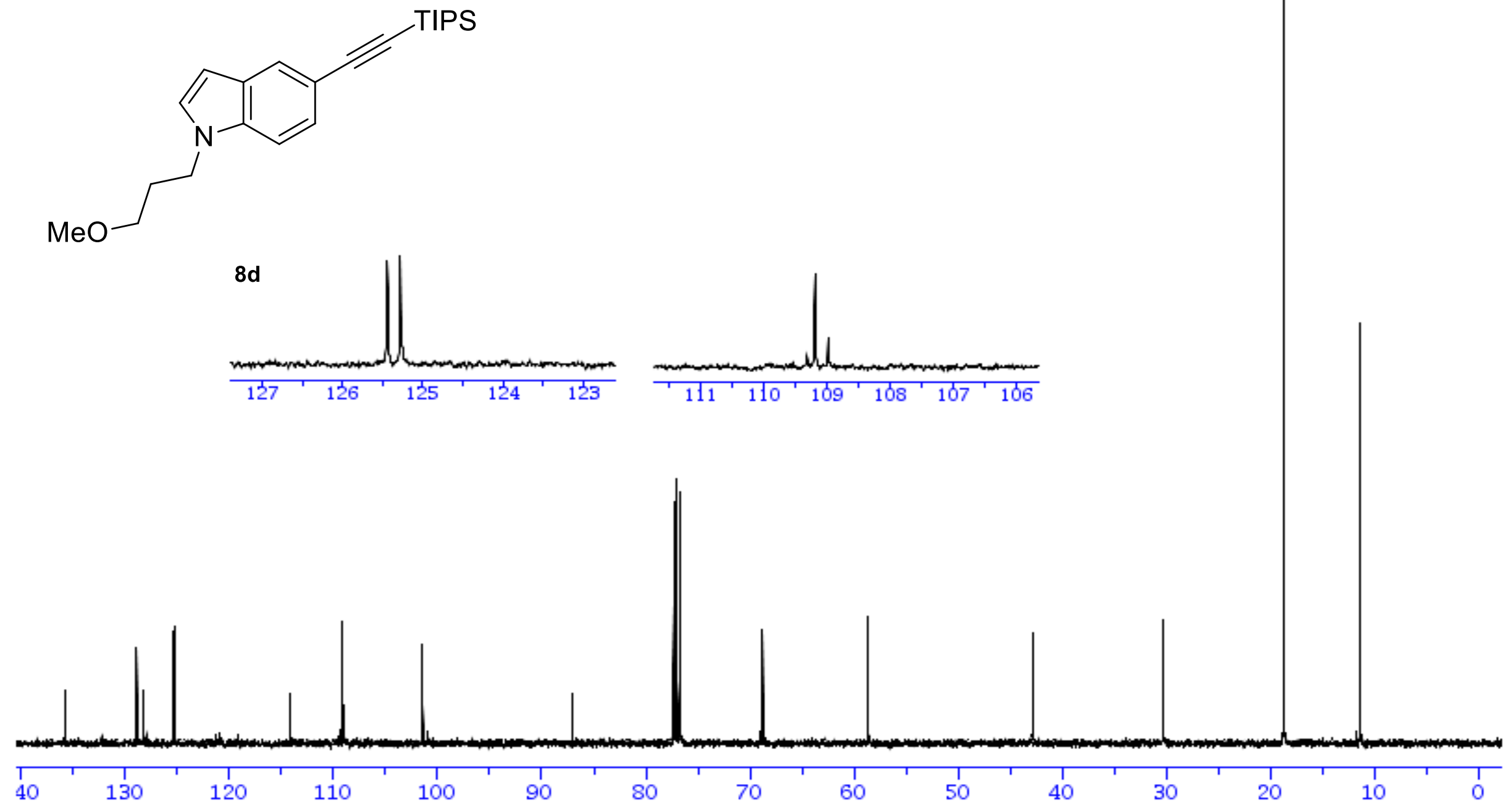


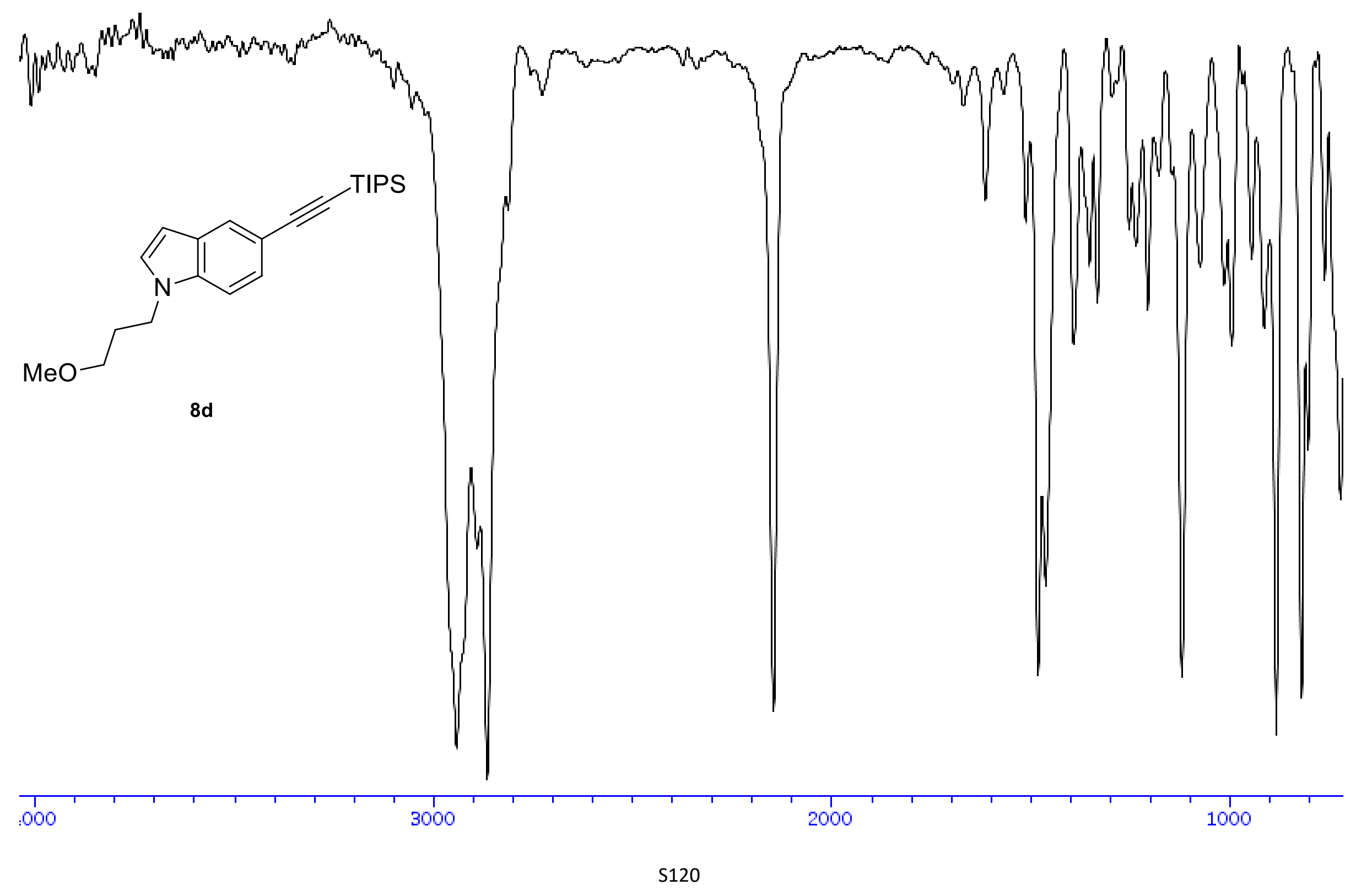


solvent: $\langle\mathrm{CDCI} 3>$

Frequency. $400.13 \mathrm{MHz}$

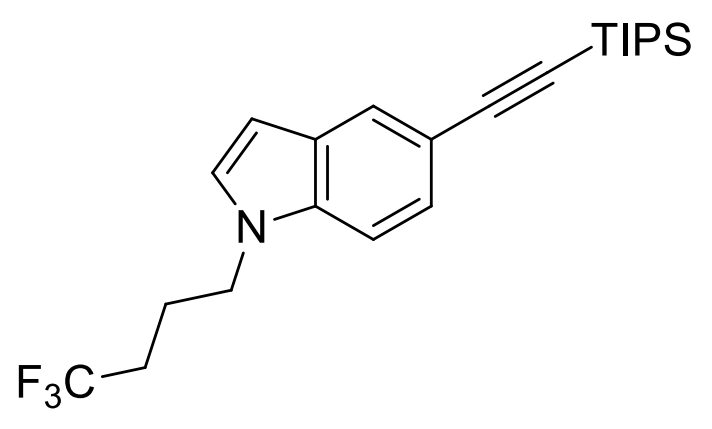

$8 e$
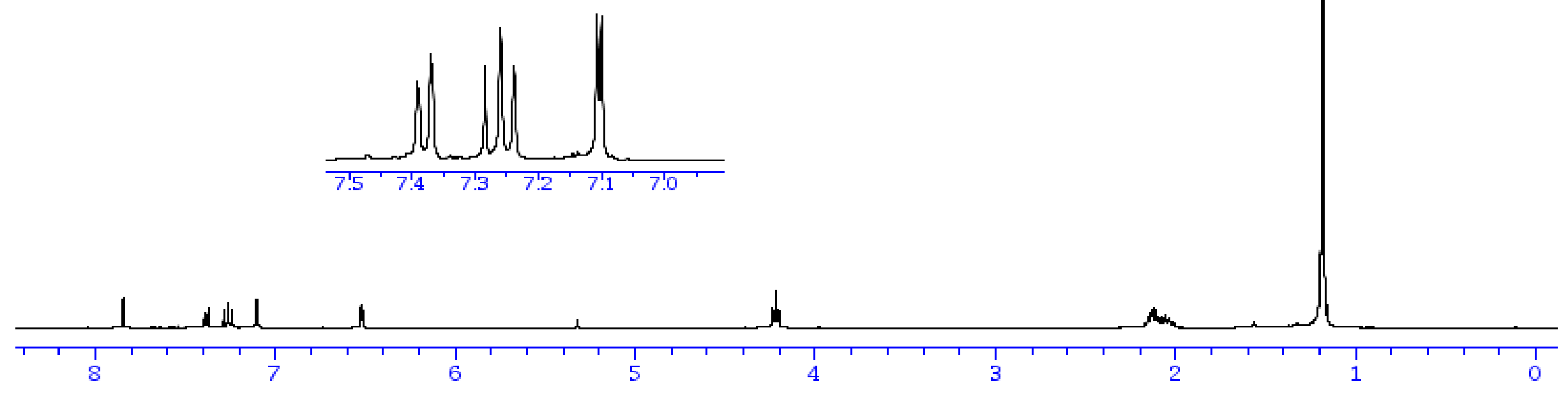
solvent: $\langle\mathrm{CDCl} 3\rangle$

Frequency. 100.612769M Hz

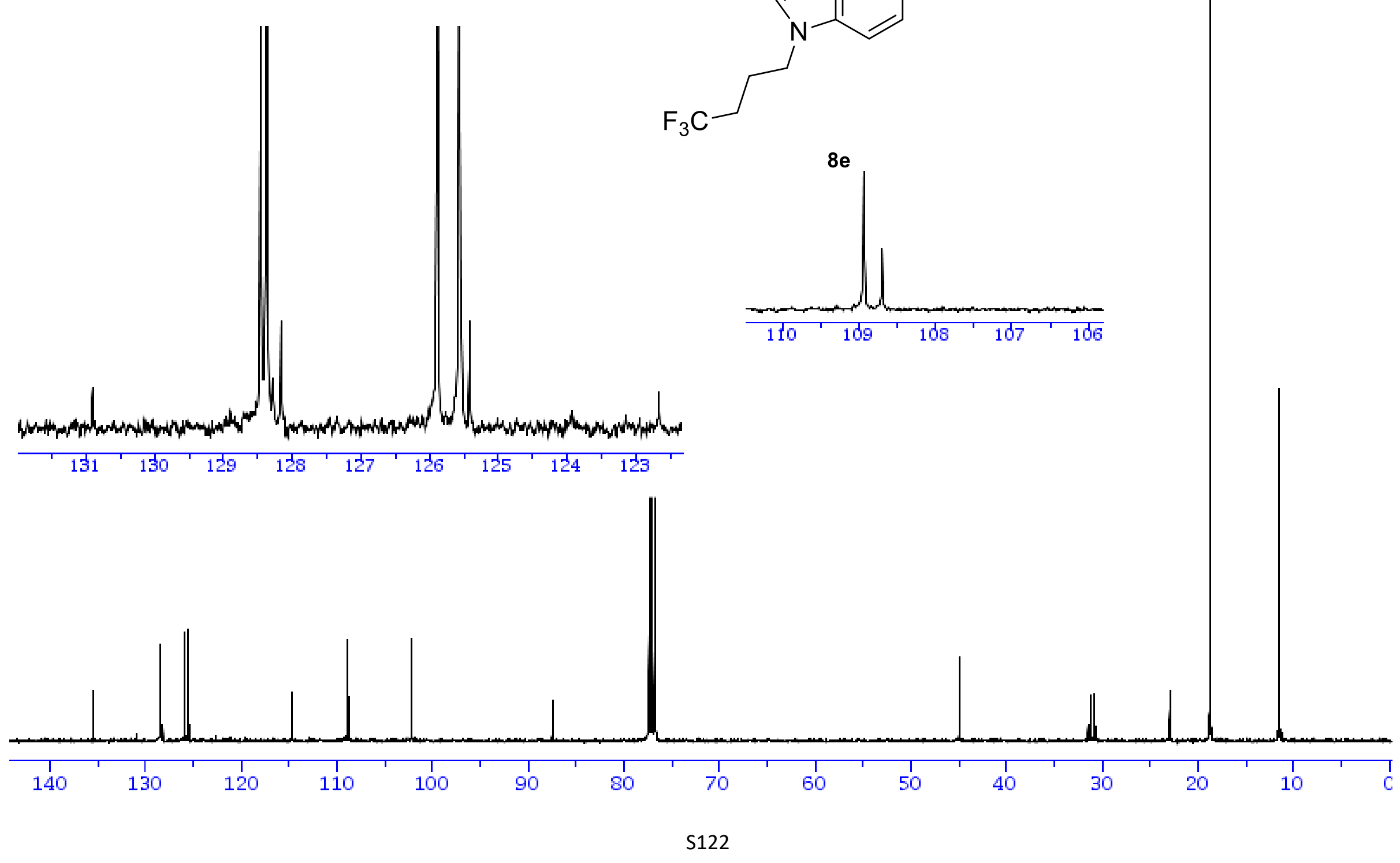




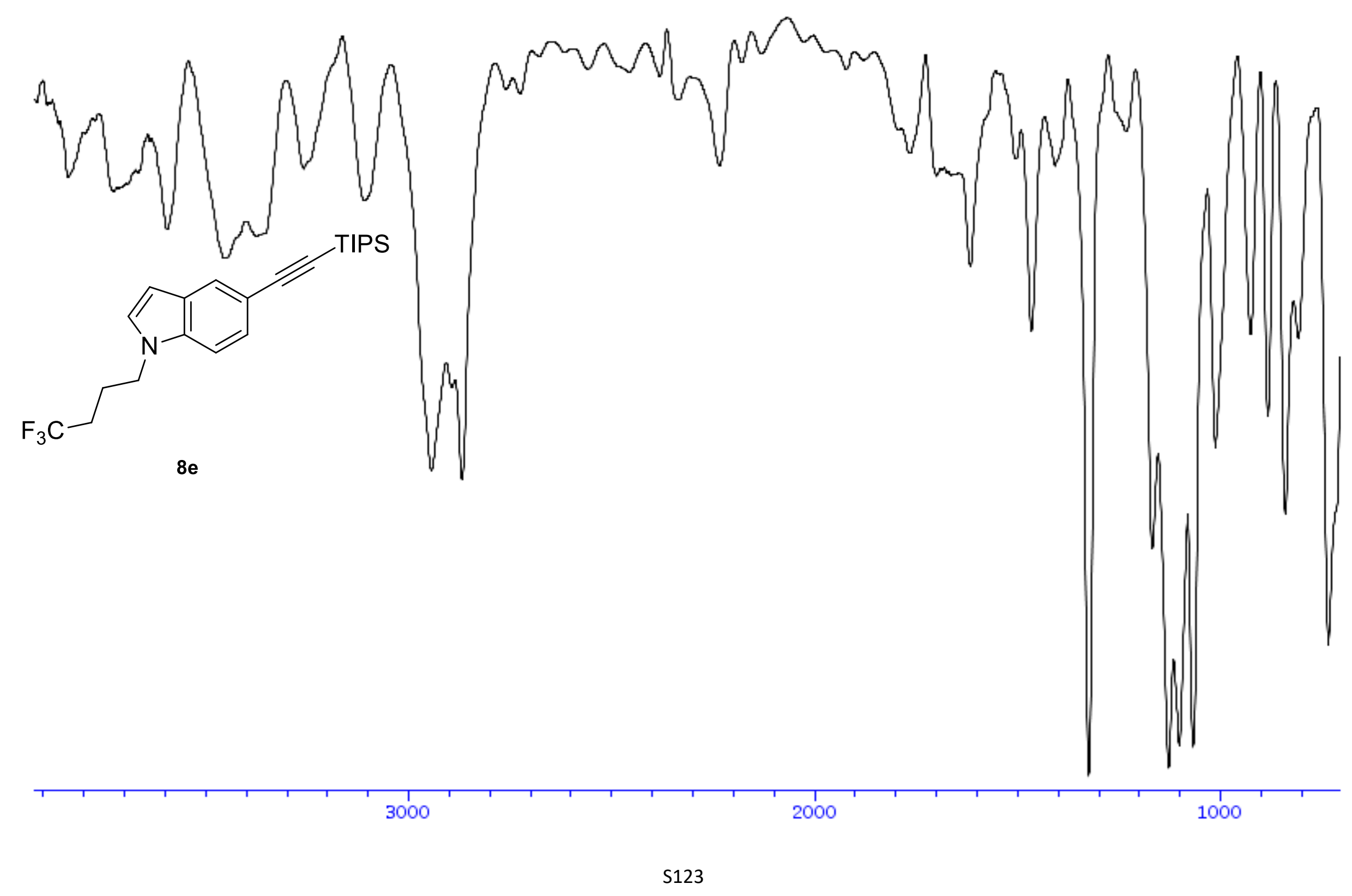


solvent: $<\mathrm{CDCI} 3>$

Frequency. $400.13 \mathrm{MHz}$

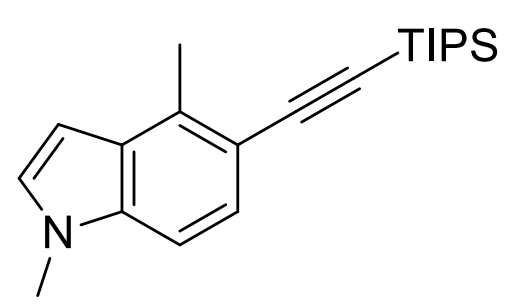

$8 f$

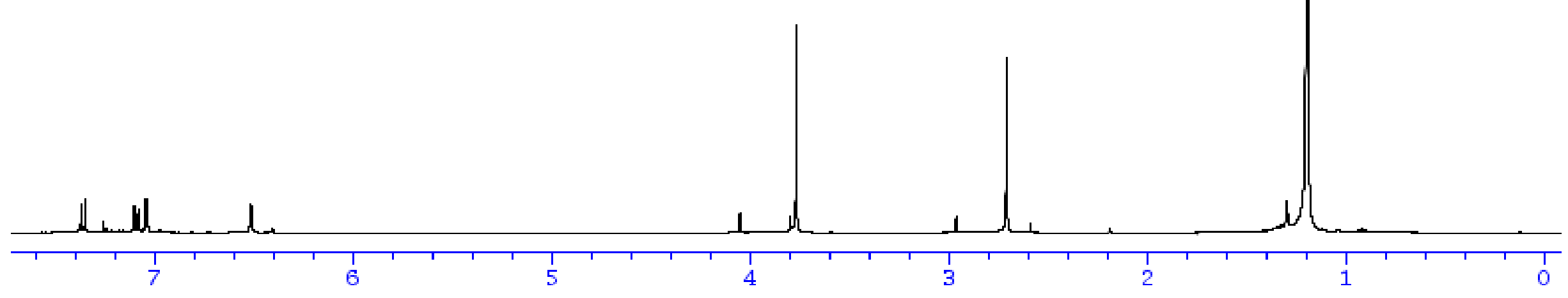


solvent: $\langle\mathrm{CDCl} 3\rangle$

Frequency. $100.612769 \mathrm{MHz}$

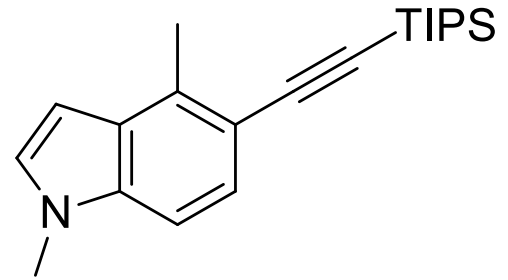

$8 f$

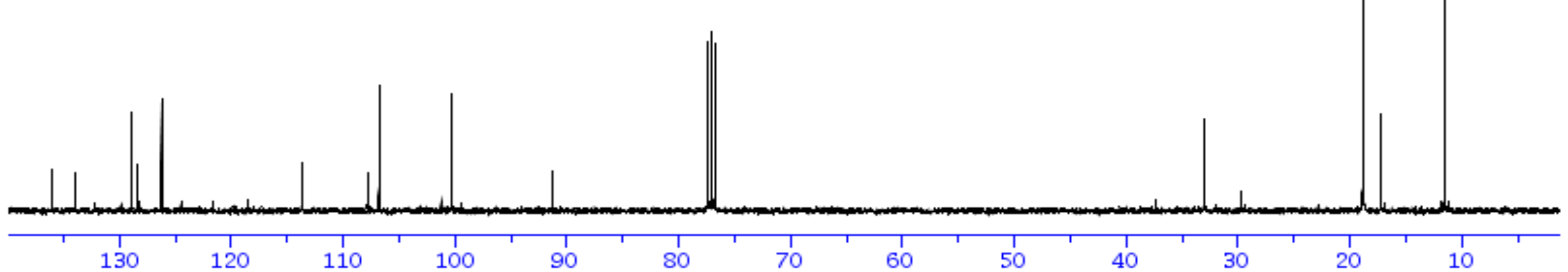




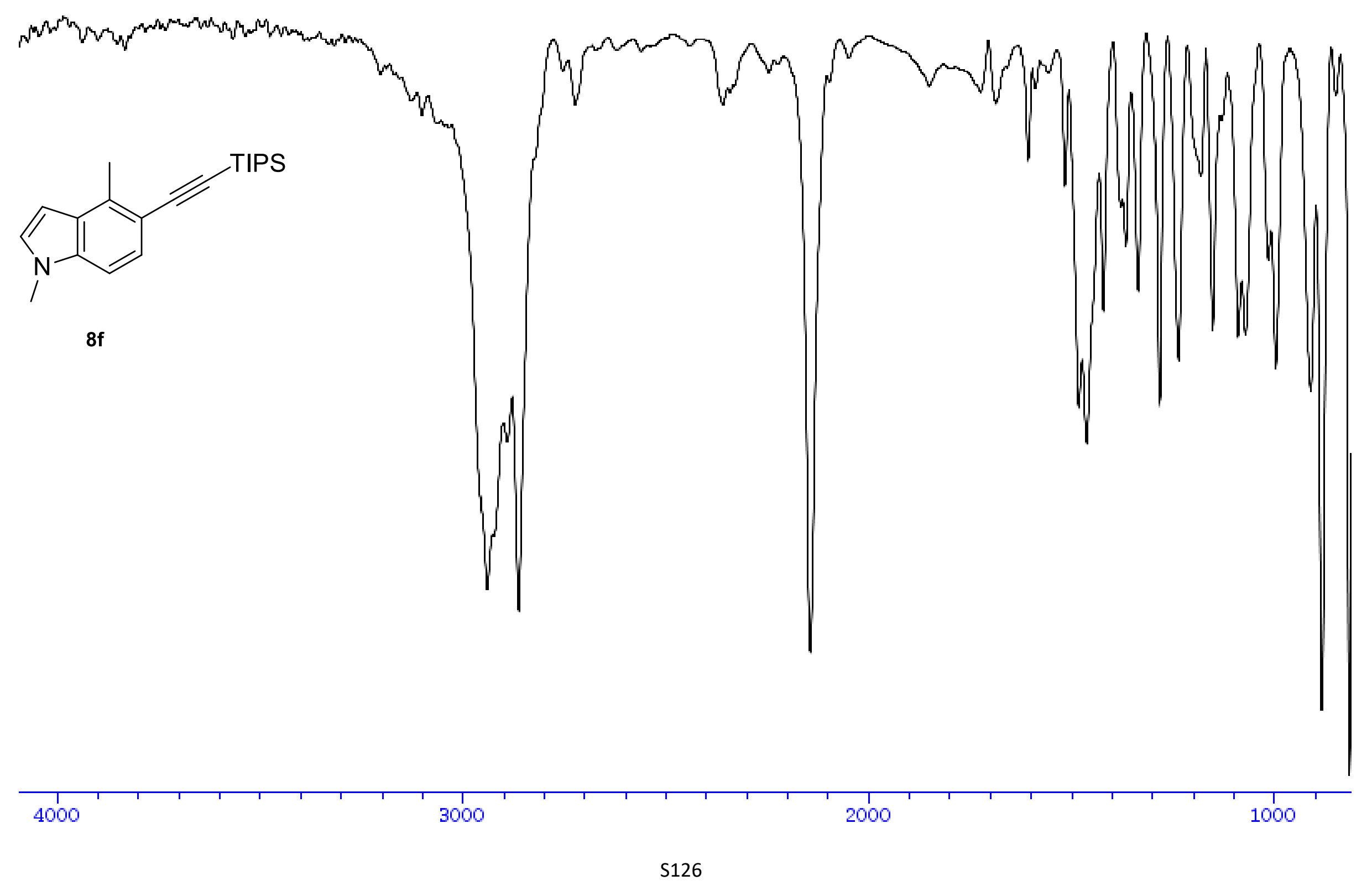


solvent: $\langle\mathrm{CDCI} 3>$

Frequency. $400.13 \mathrm{MHz}$

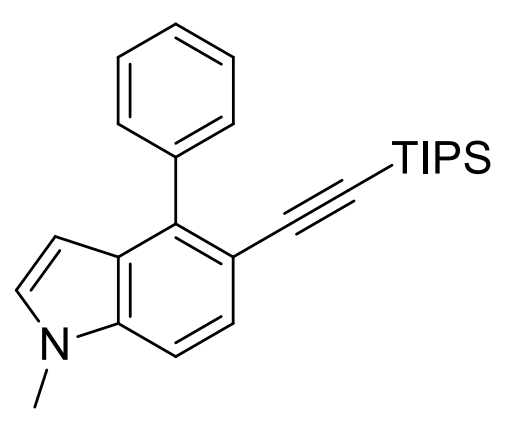

$8 g$

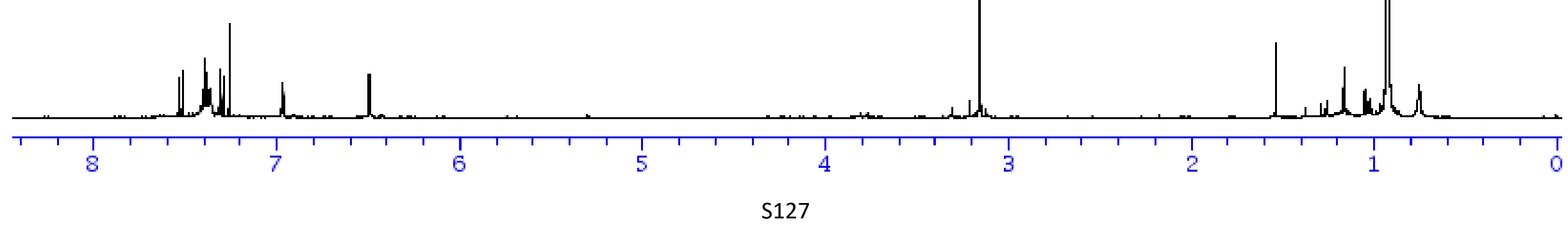


solvent: $\langle\mathrm{CDCl} 3\rangle$

Frequency. $100.612769 \mathrm{MHz}$

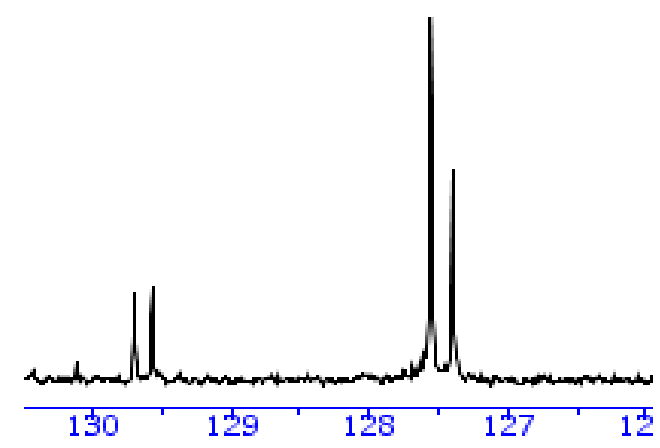

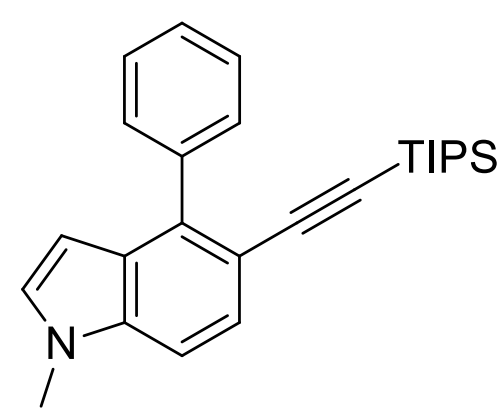

$8 g$

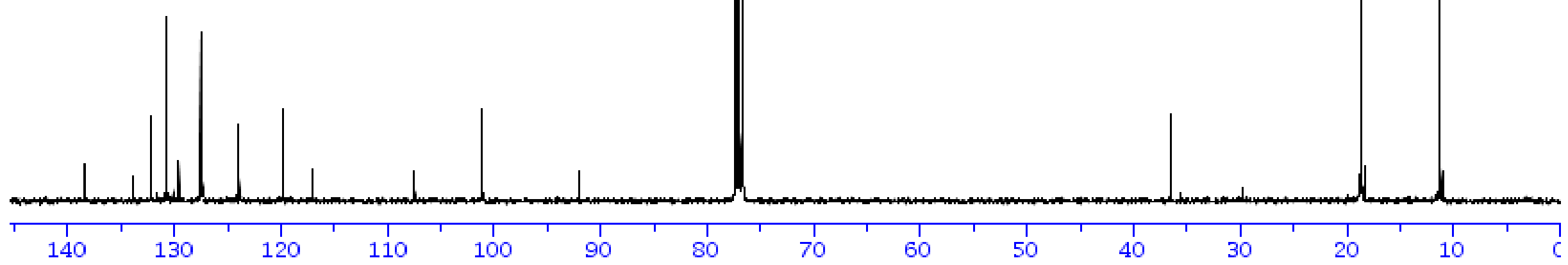




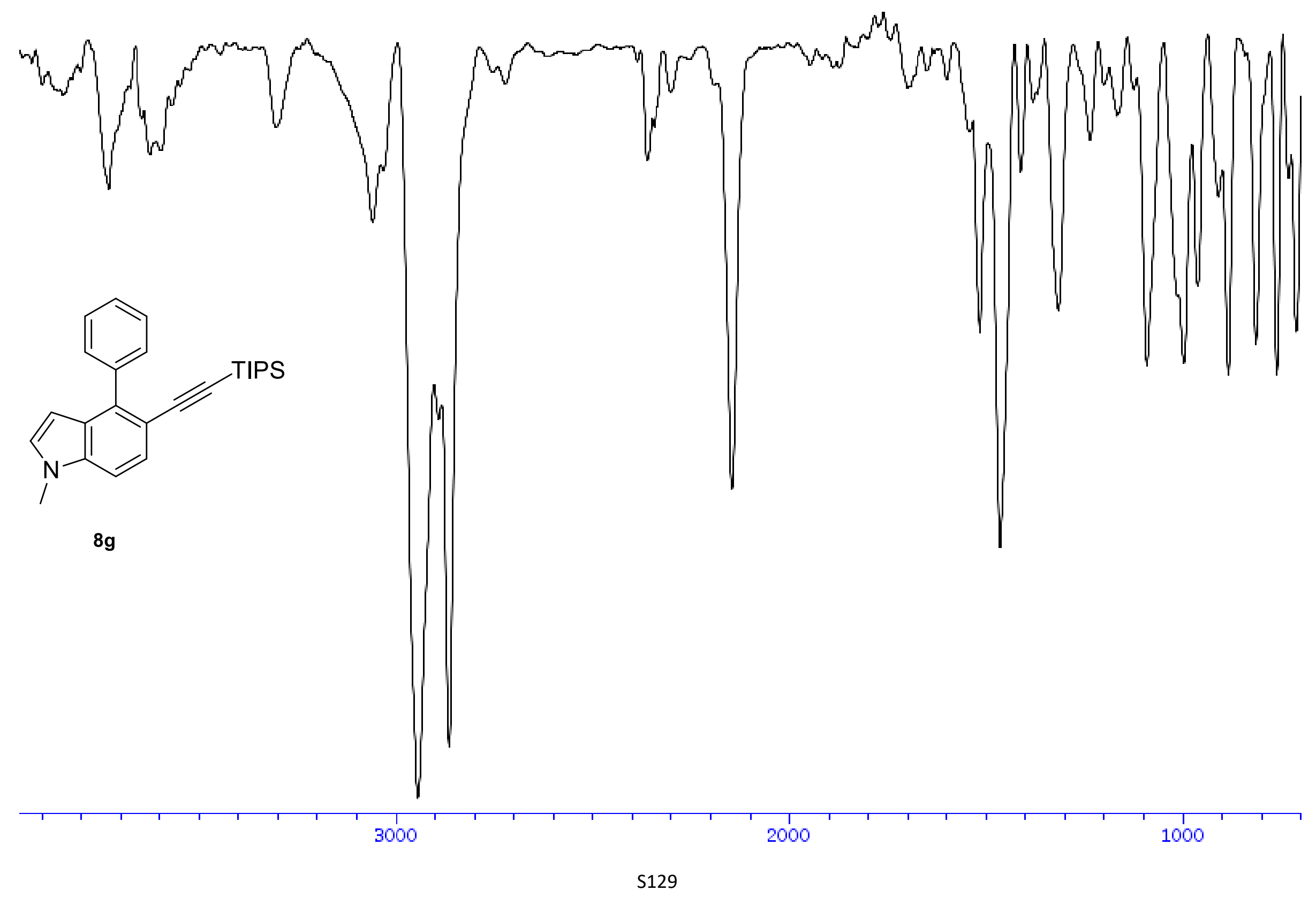


\}

solvent: $\langle\mathrm{CDCl} 3\rangle$

Frequency. $400.13 \mathrm{MHz}$

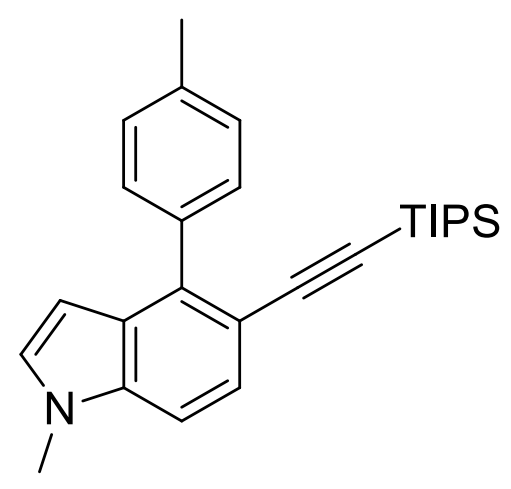

8h

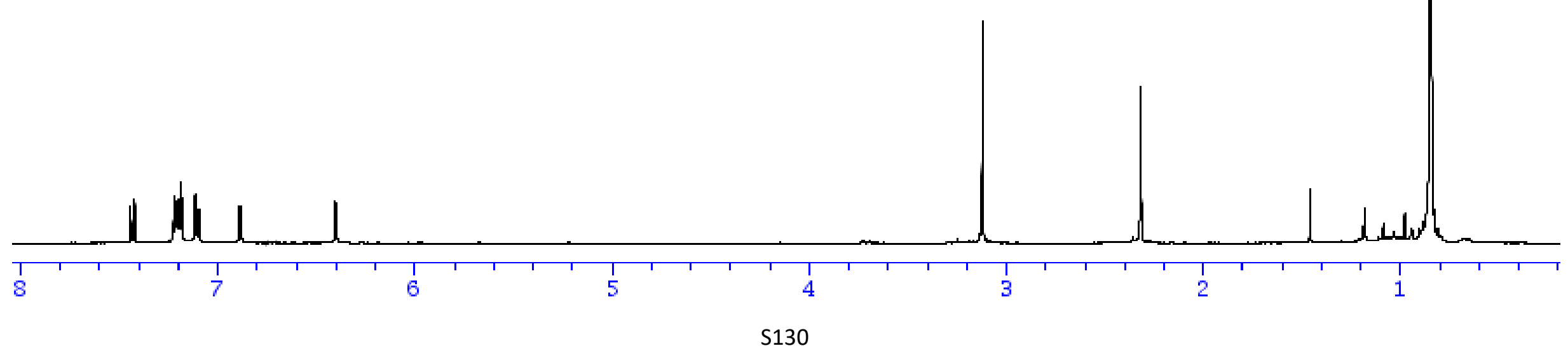


solvent: $\langle\mathrm{CDCl} 3>$

Frequency. 100.612769M Hz

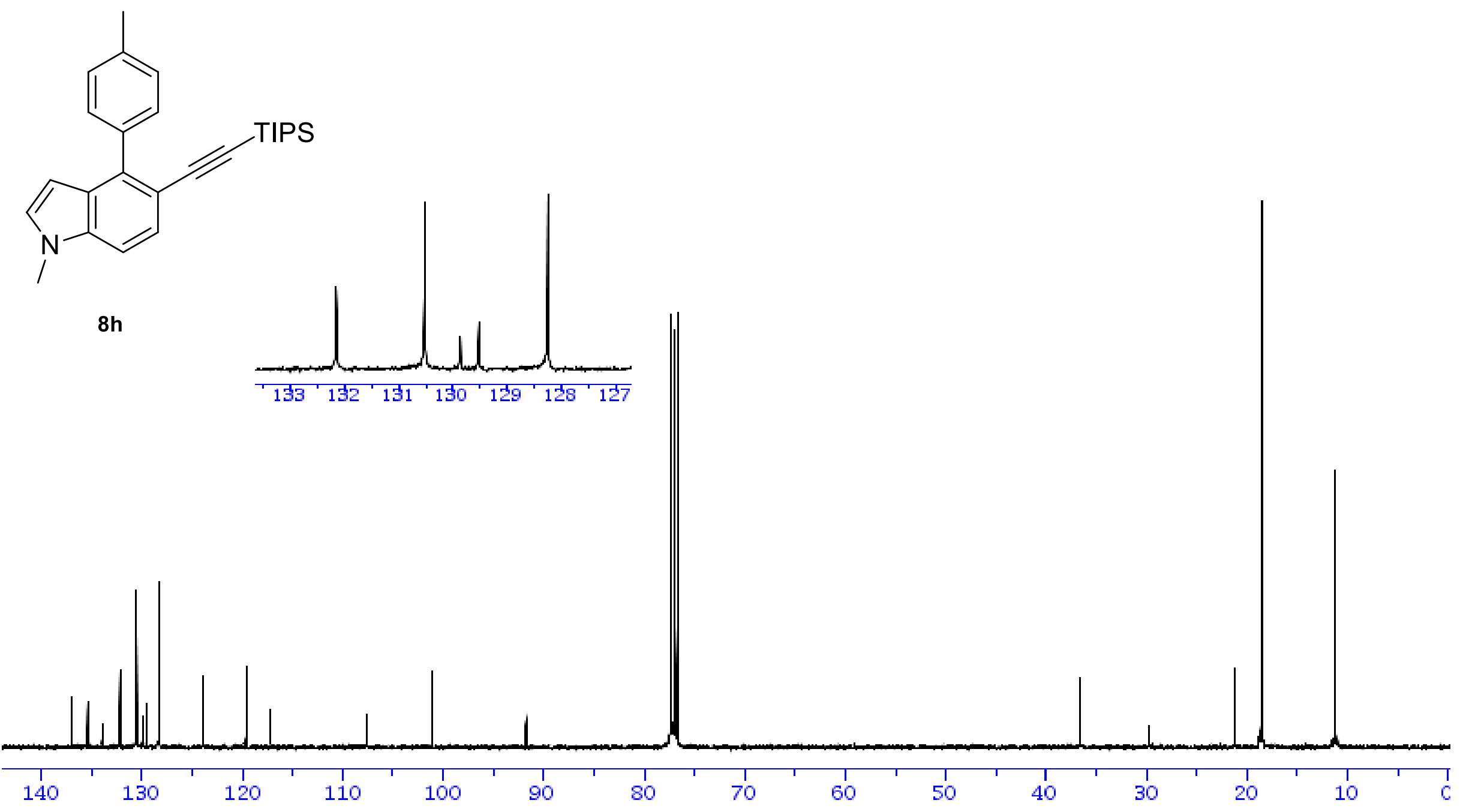




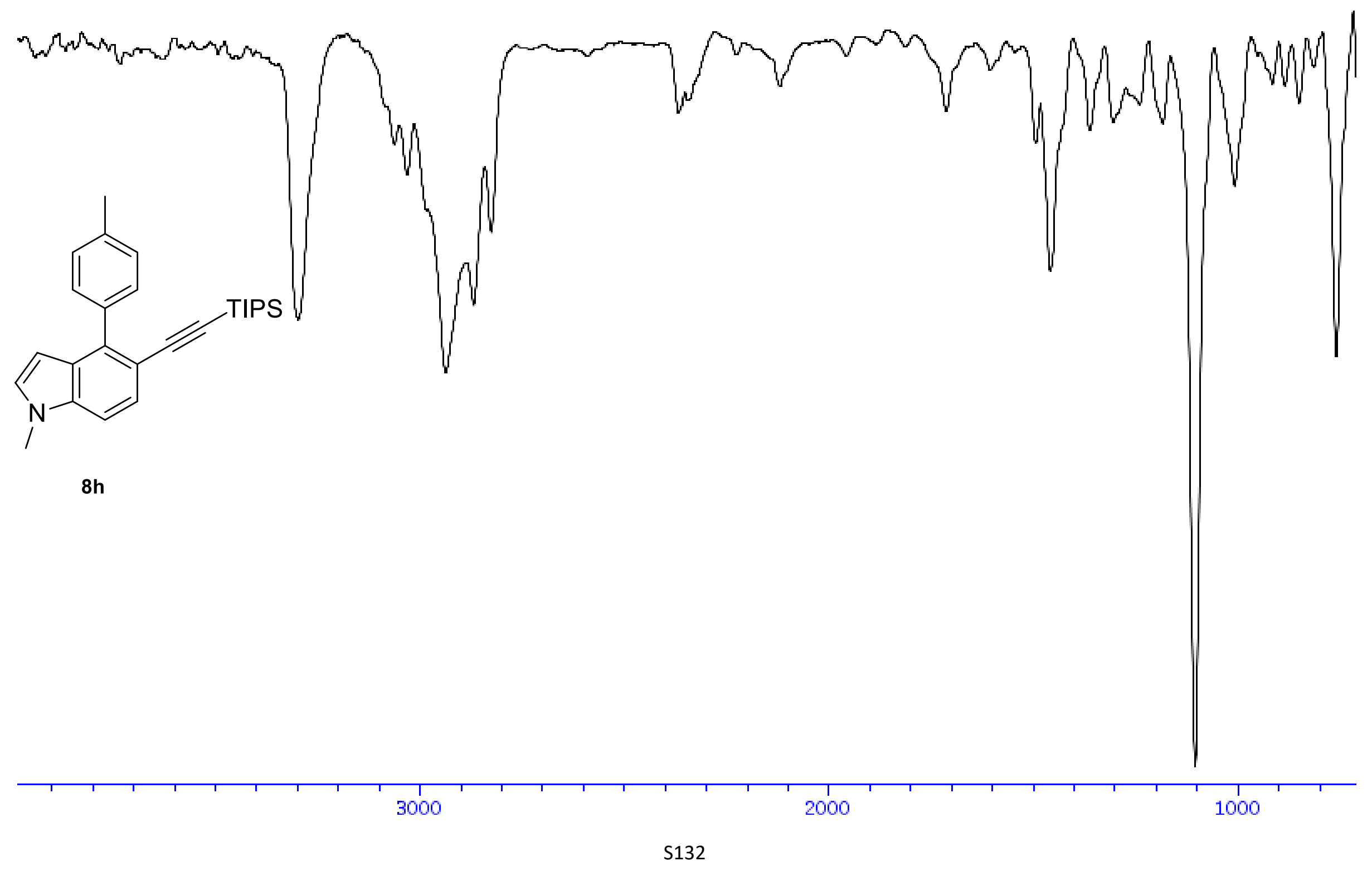


solvent:<CDCI3>

Frequency: $400.13 \mathrm{MHz}$

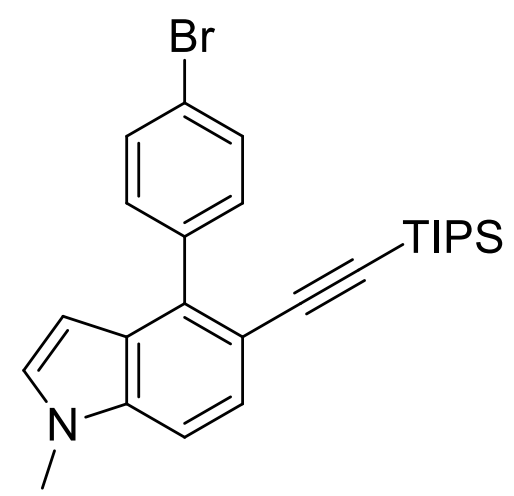

$8 \mathbf{i}$

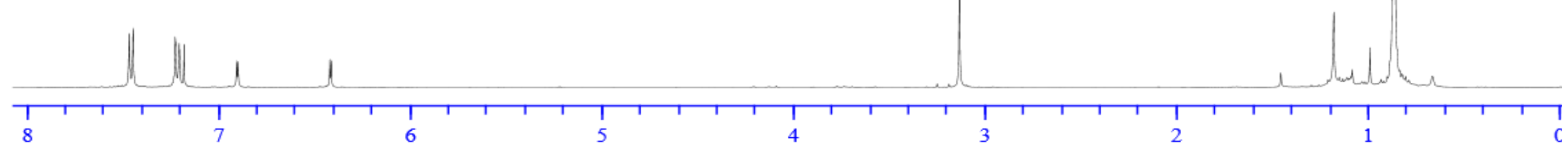


solvent:<CDCI3>

Frequency: $100.612769 \mathrm{MHz}$
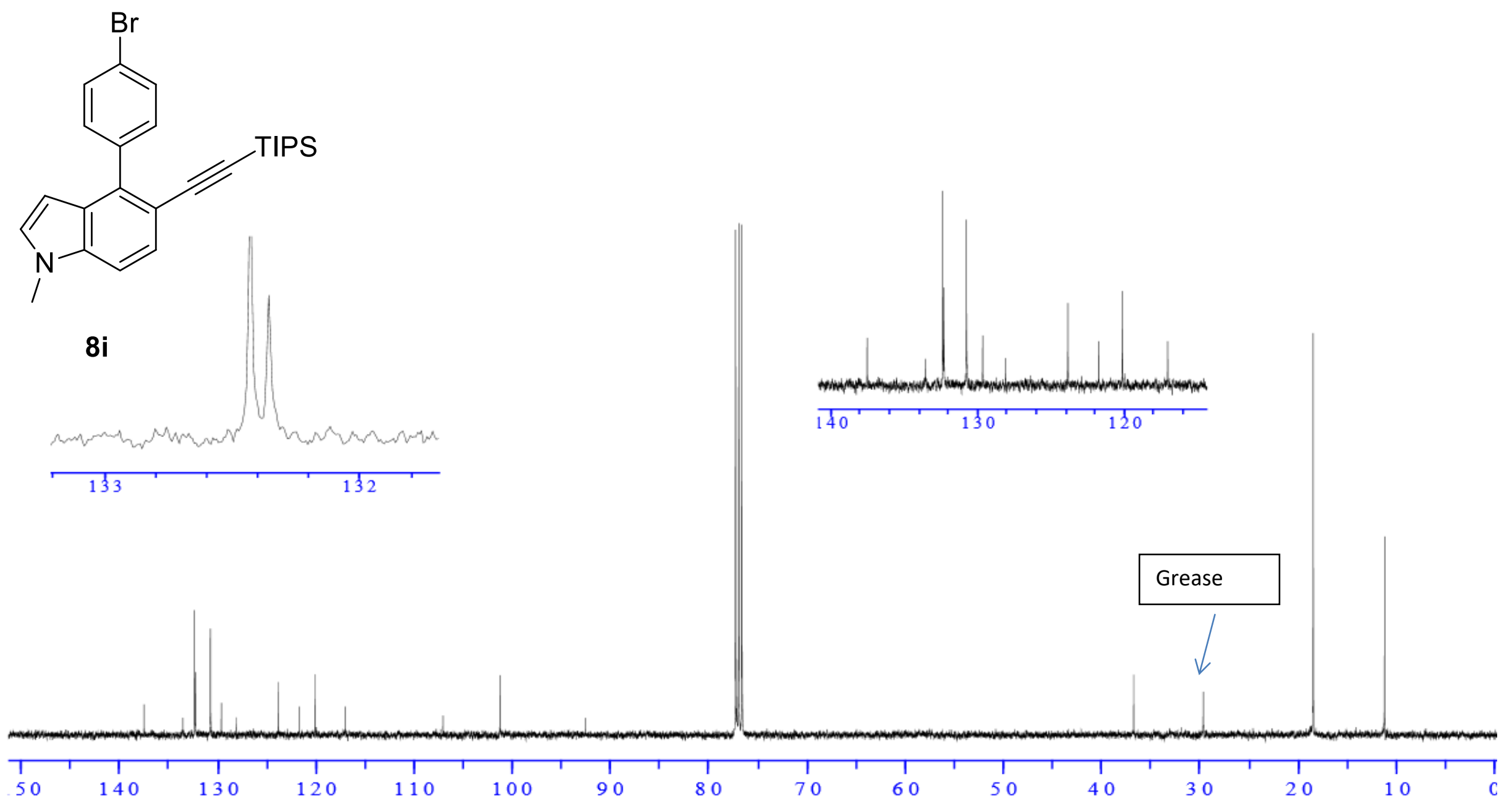


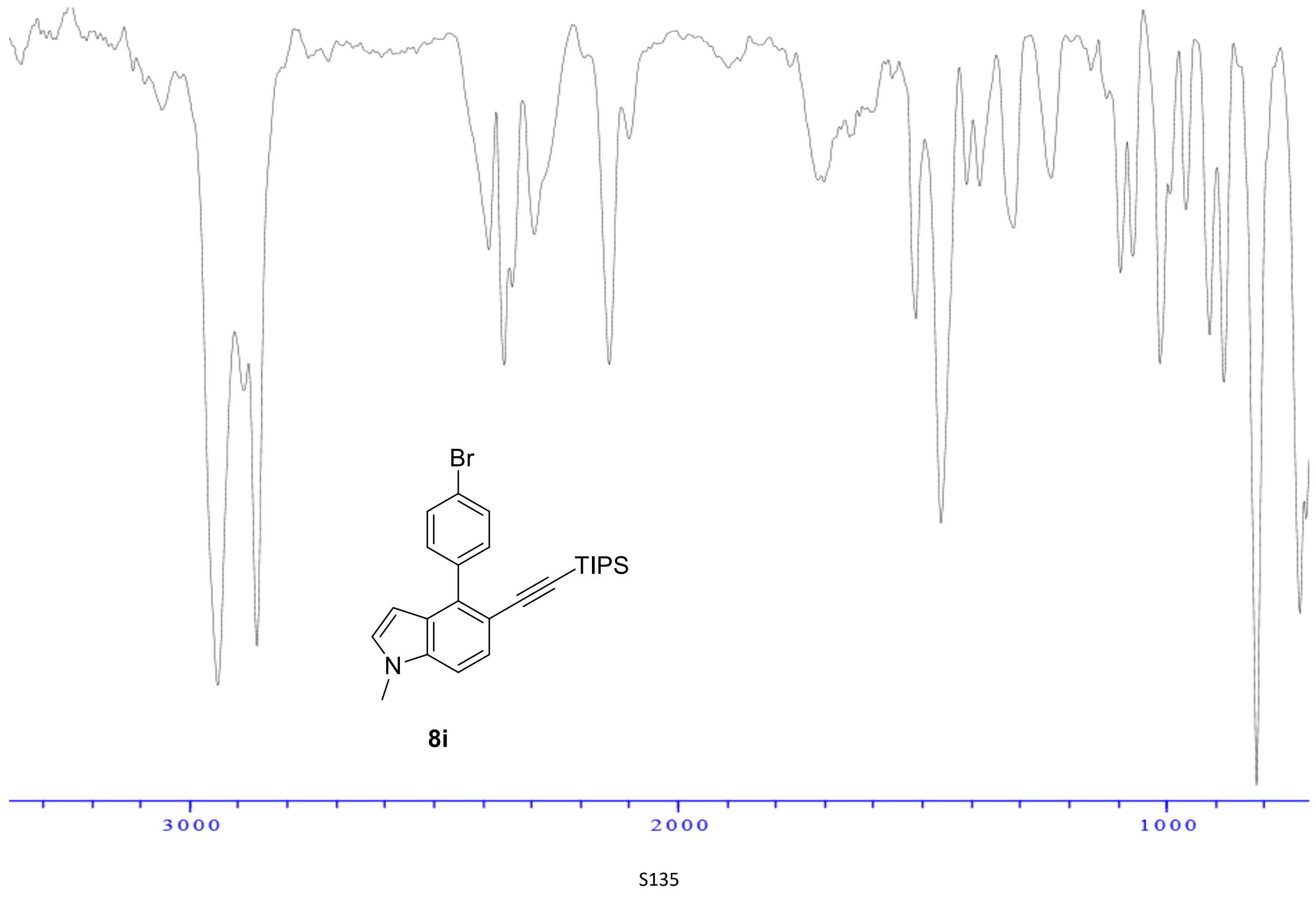


solvent: $\langle\mathrm{CDCl} 3\rangle$

Frequency $400.13 \mathrm{MHz}$

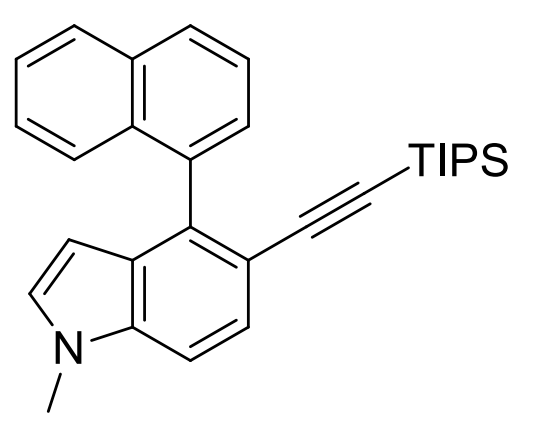

8j

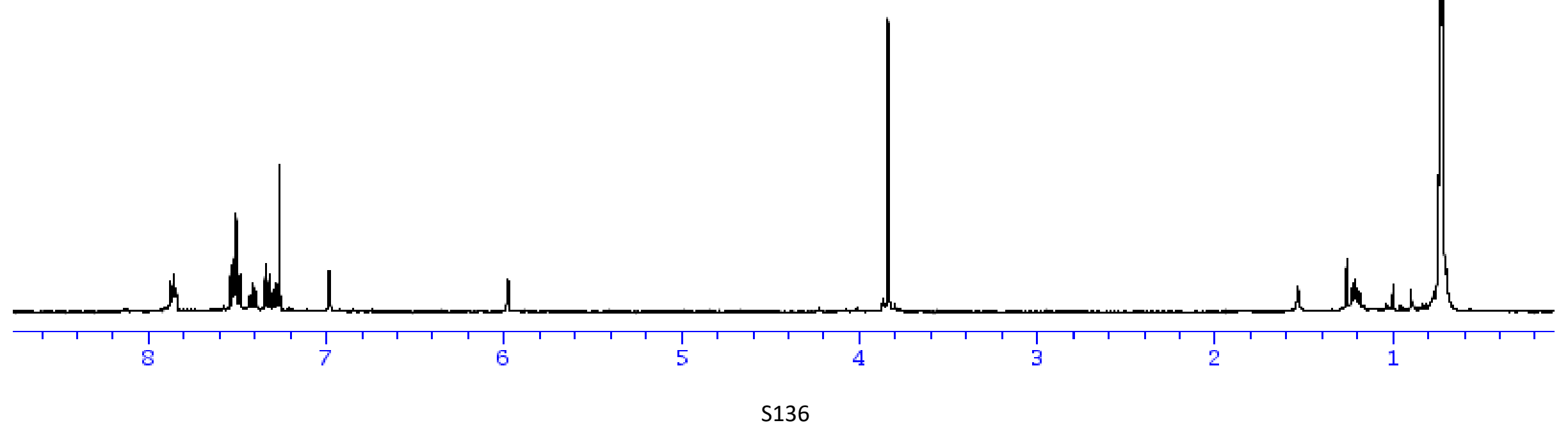


solvent: $<\mathrm{CDCI} 3>$

Frequency. $100.612769 \mathrm{MHz}$

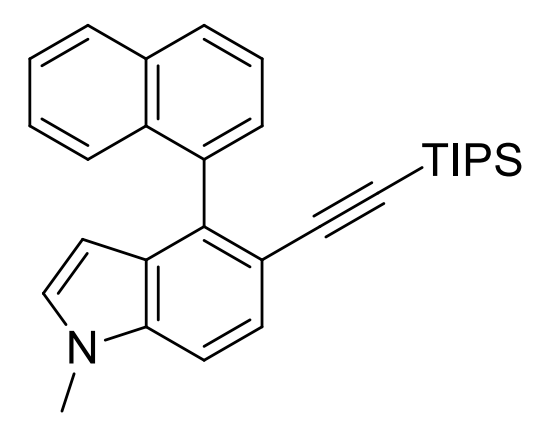

8j
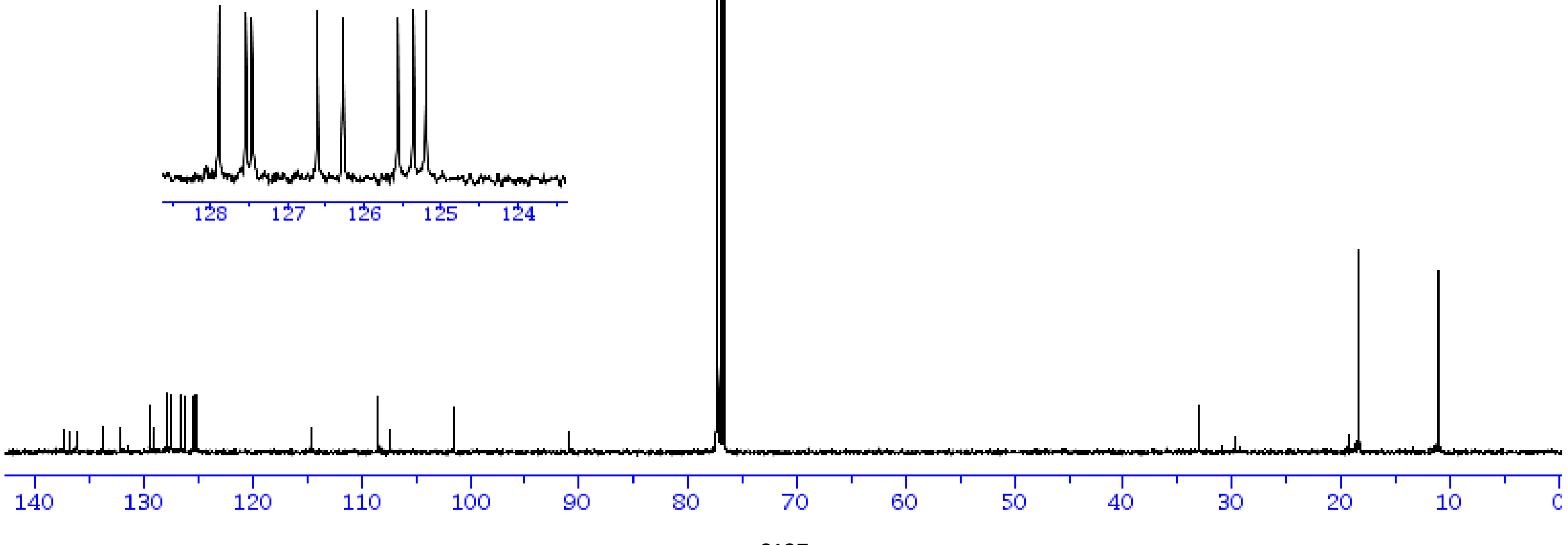


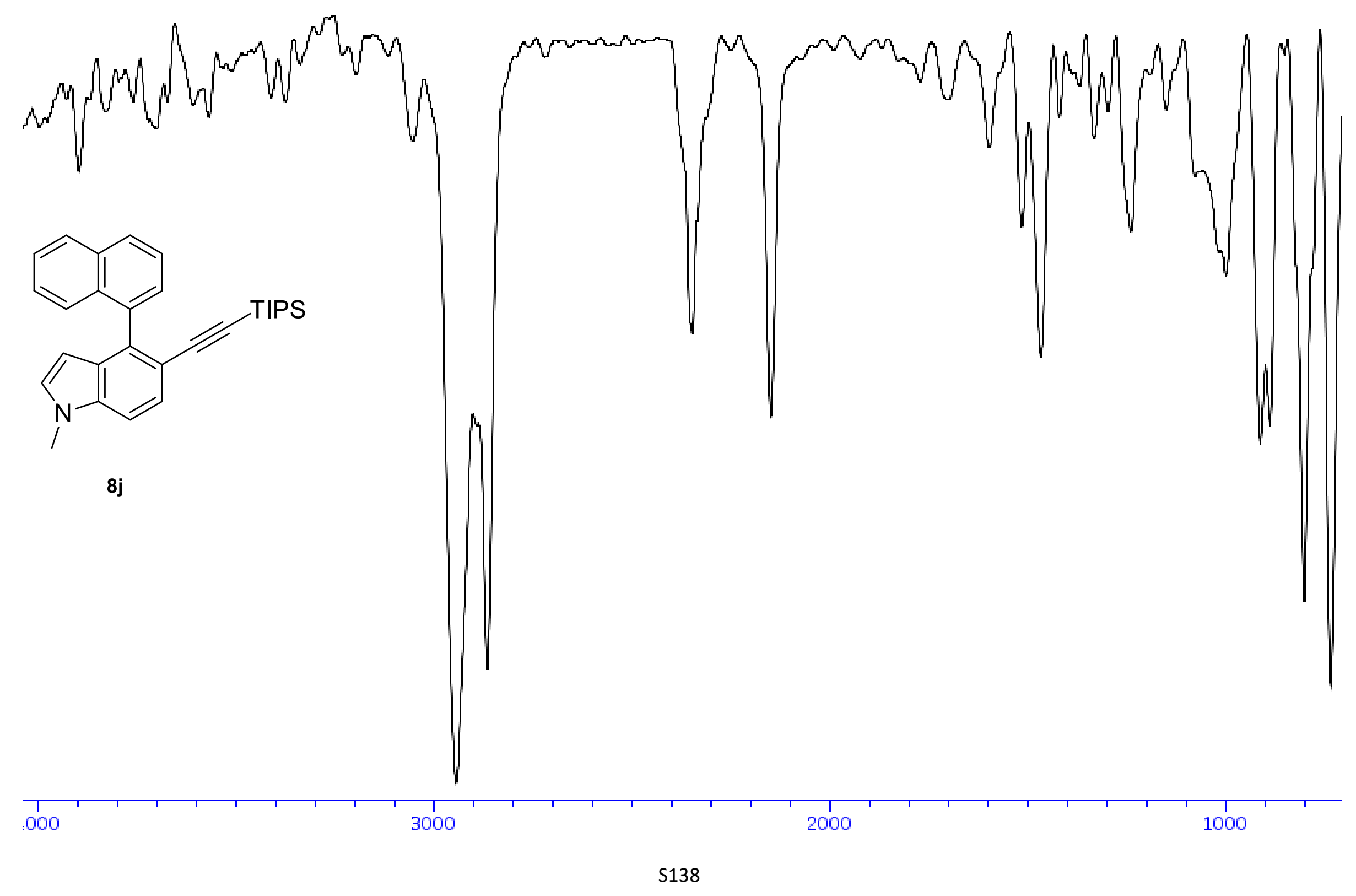


solvent: $\langle\mathrm{CDCl} 3>$

Frequency. $400.13 \mathrm{MHz}$

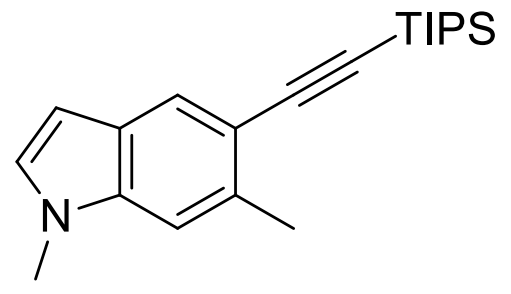

8k

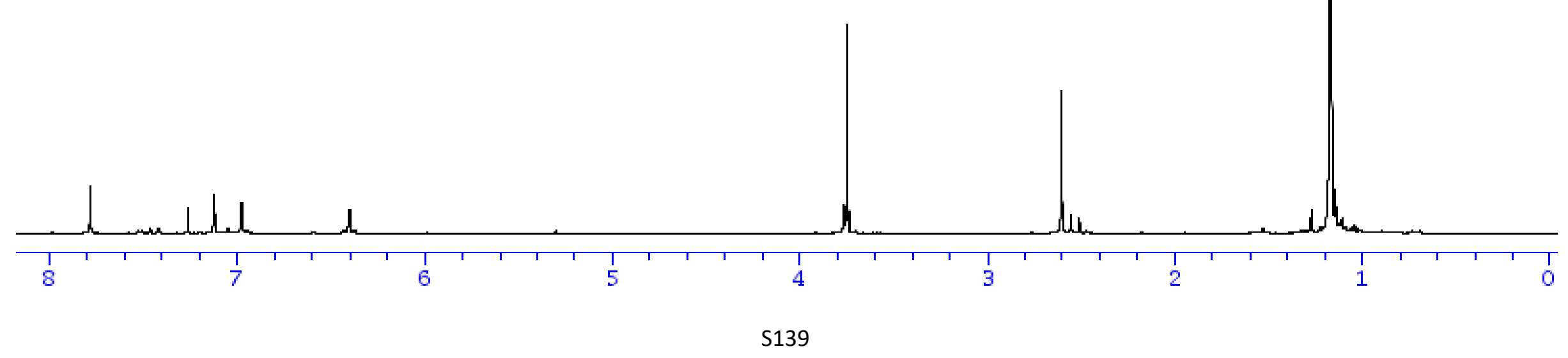


solvent: $\langle\mathrm{CDCl} 3\rangle$

Frequency. $100.612769 \mathrm{MHz}$

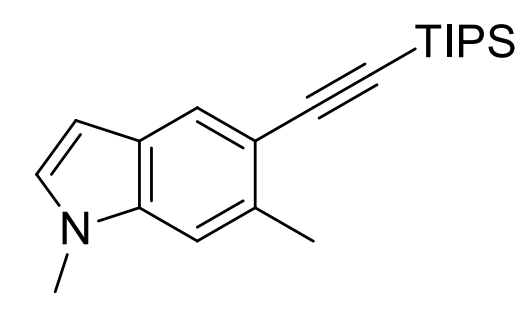

8k

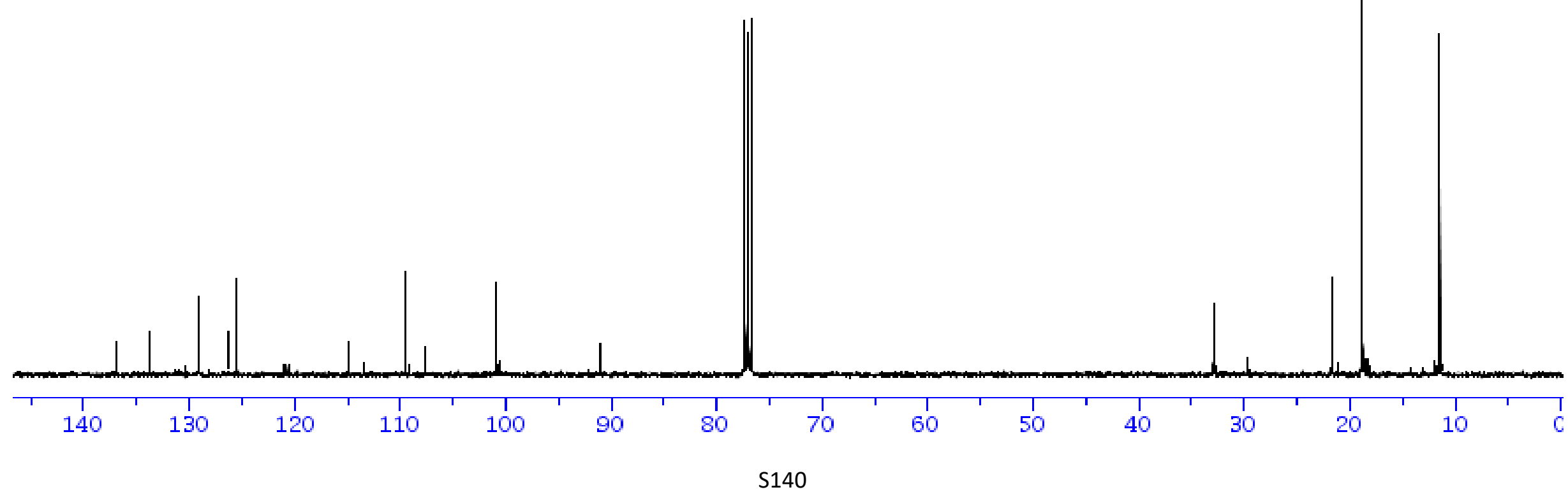




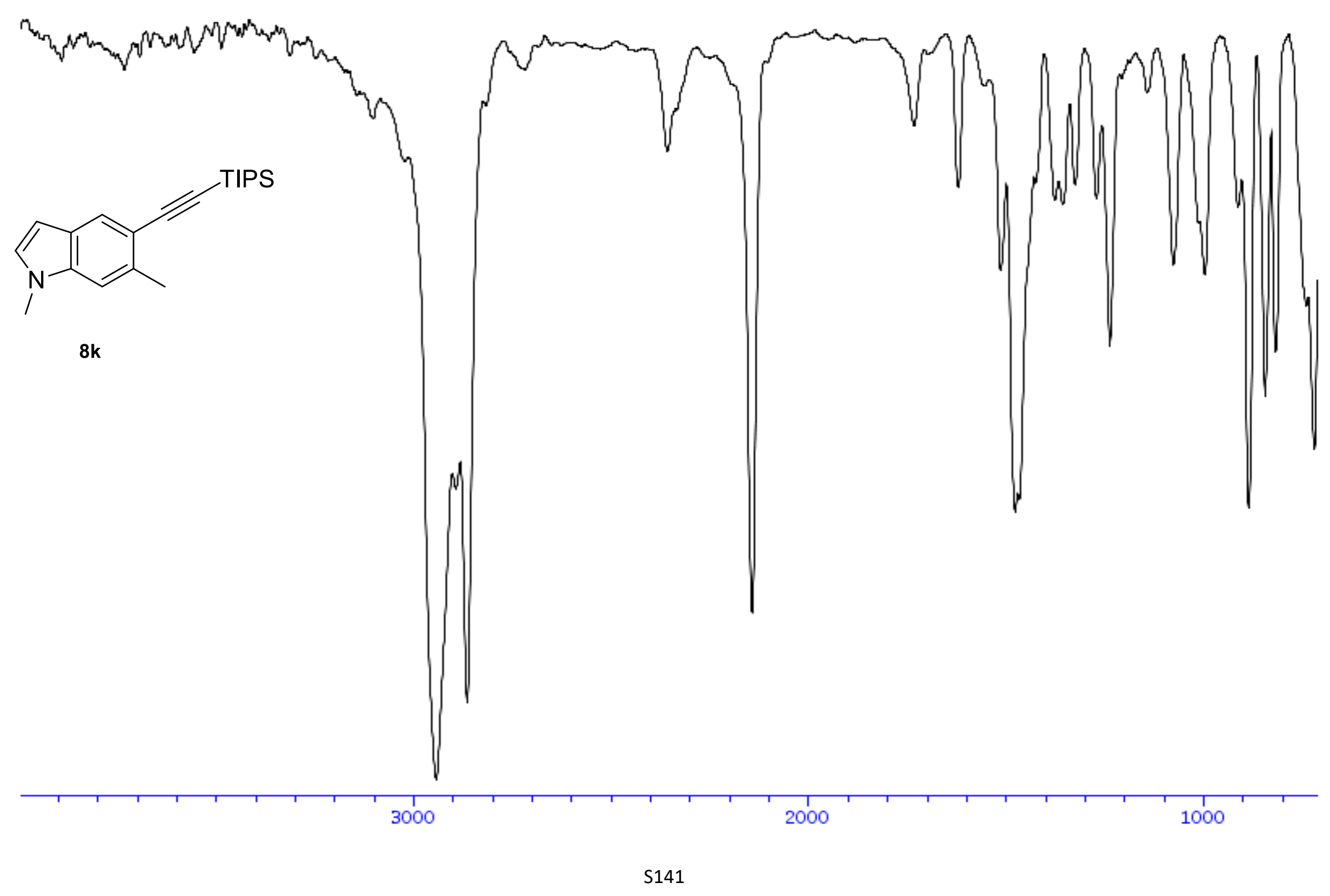


solvent: $<C D C I 3>$

Frequency. $400.13 \mathrm{MHz}$

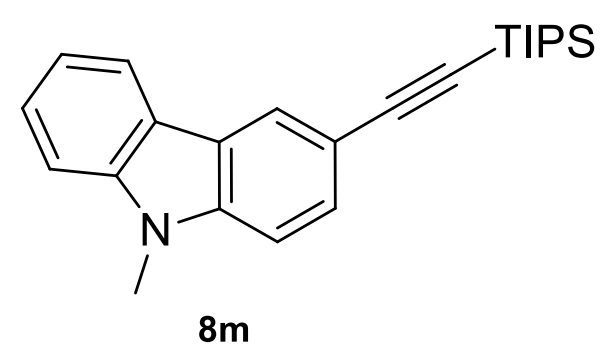

$8 m$

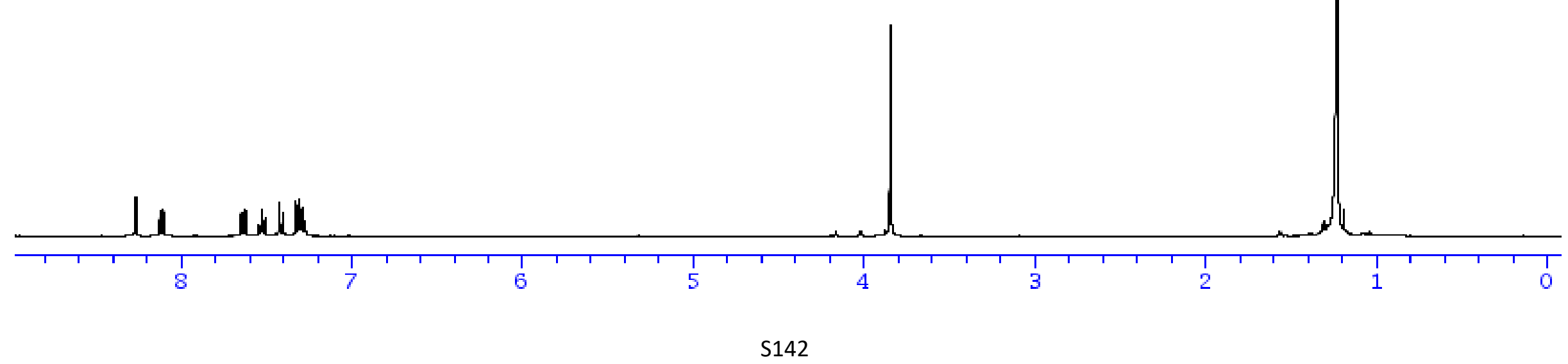


solvent: $\langle\mathrm{CDCI} 3\rangle$

Frequency. $100.612769 \mathrm{MHz}$
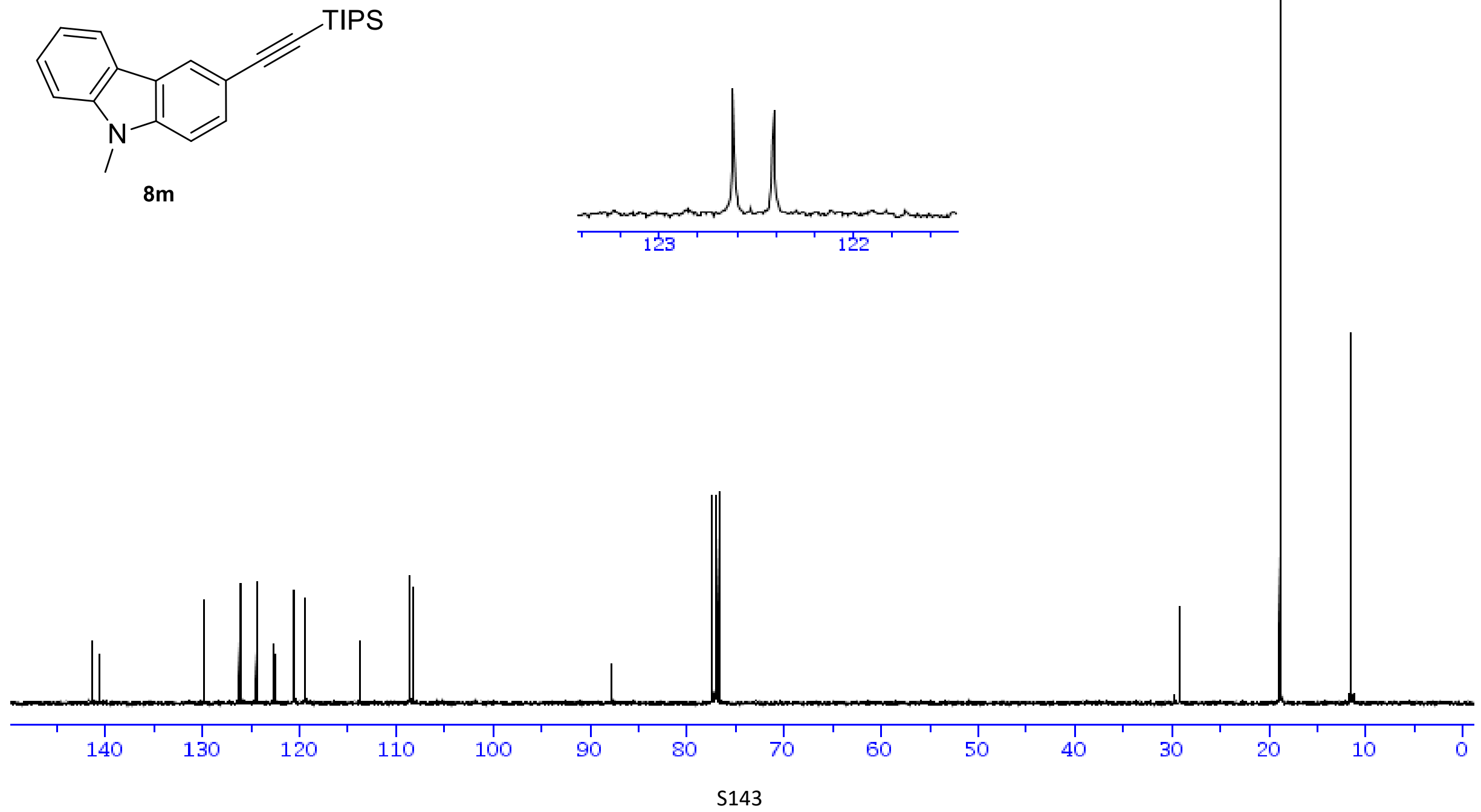


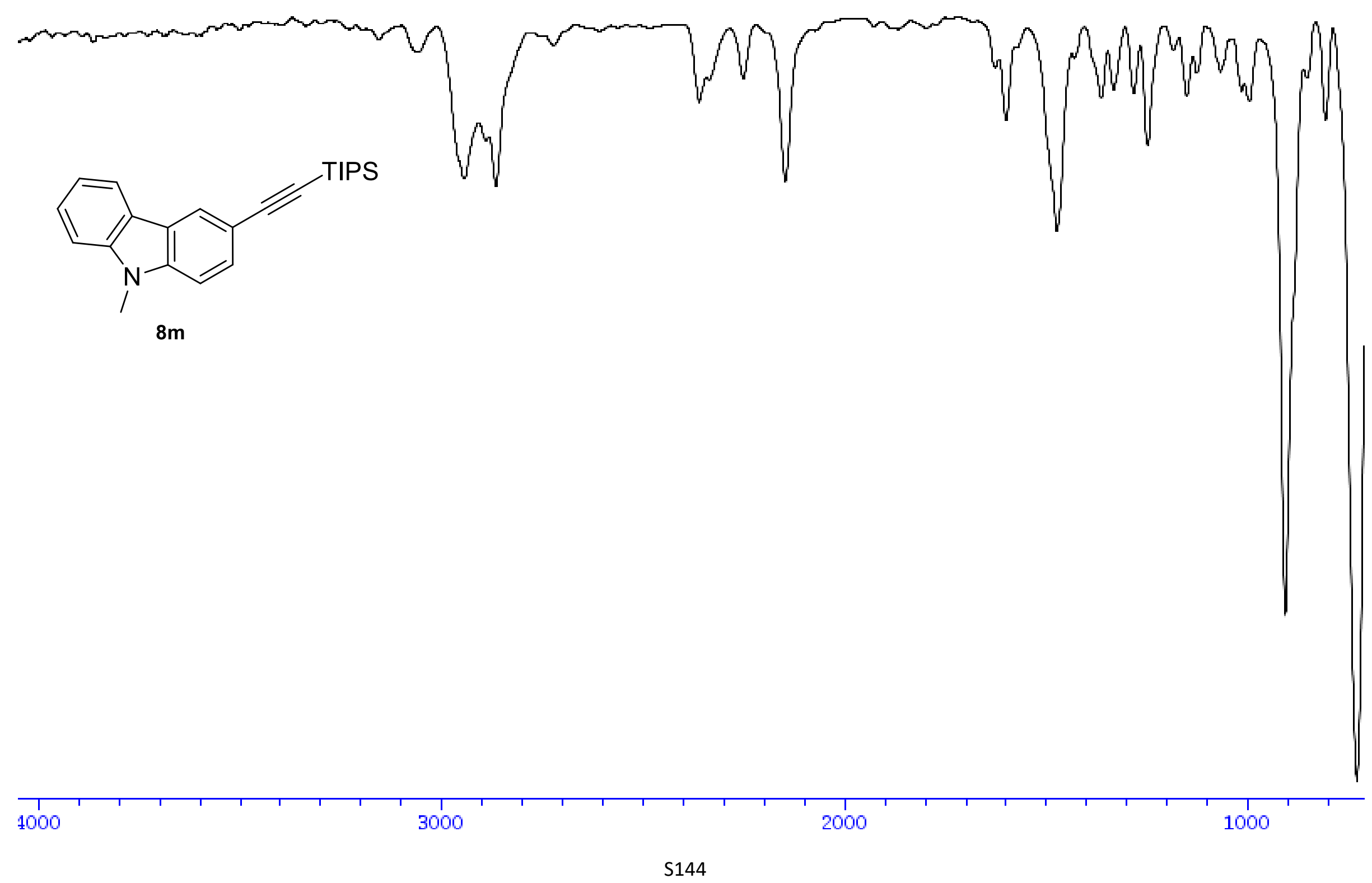


solvent: $<\mathrm{CDCI} 3>$

Frequency. $400.13 \mathrm{MHz}$

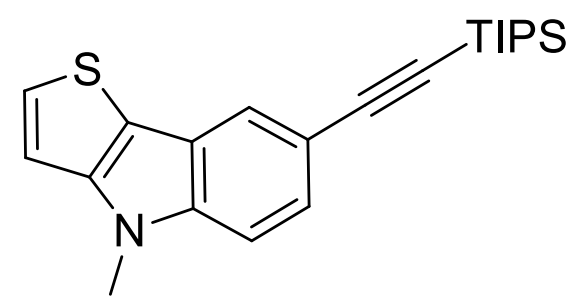

$8 n$

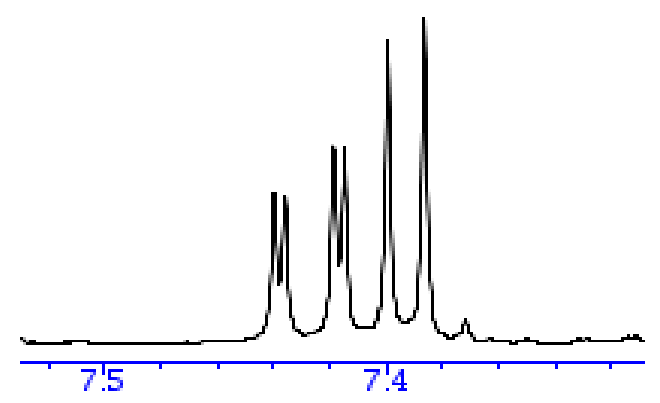

$\frac{1}{+1+1}$ 
solvent: $\langle\mathrm{CDCI}$ >

Frequency. $100.612769 \mathrm{MHz}$

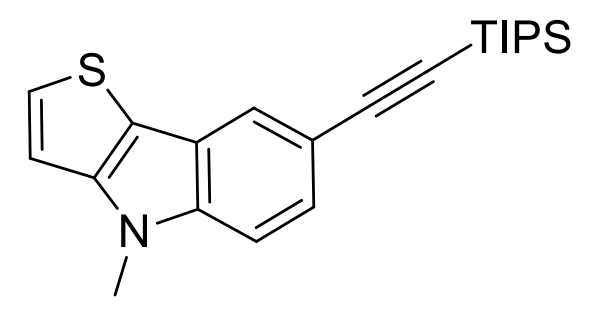

$8 n$

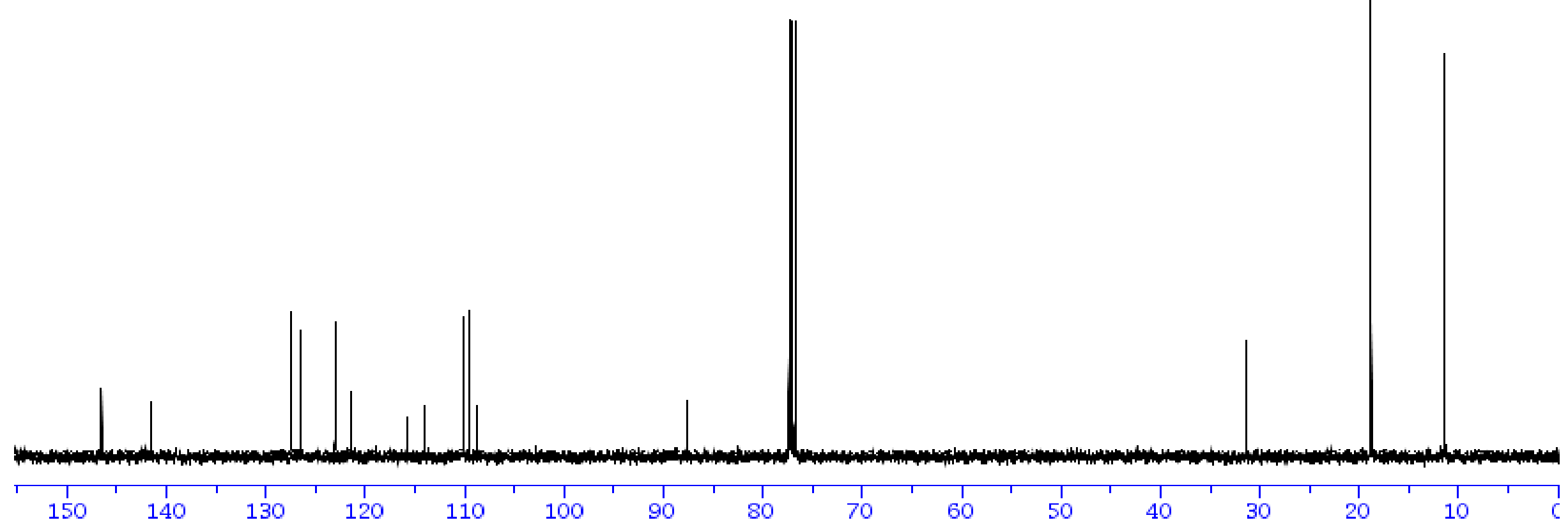




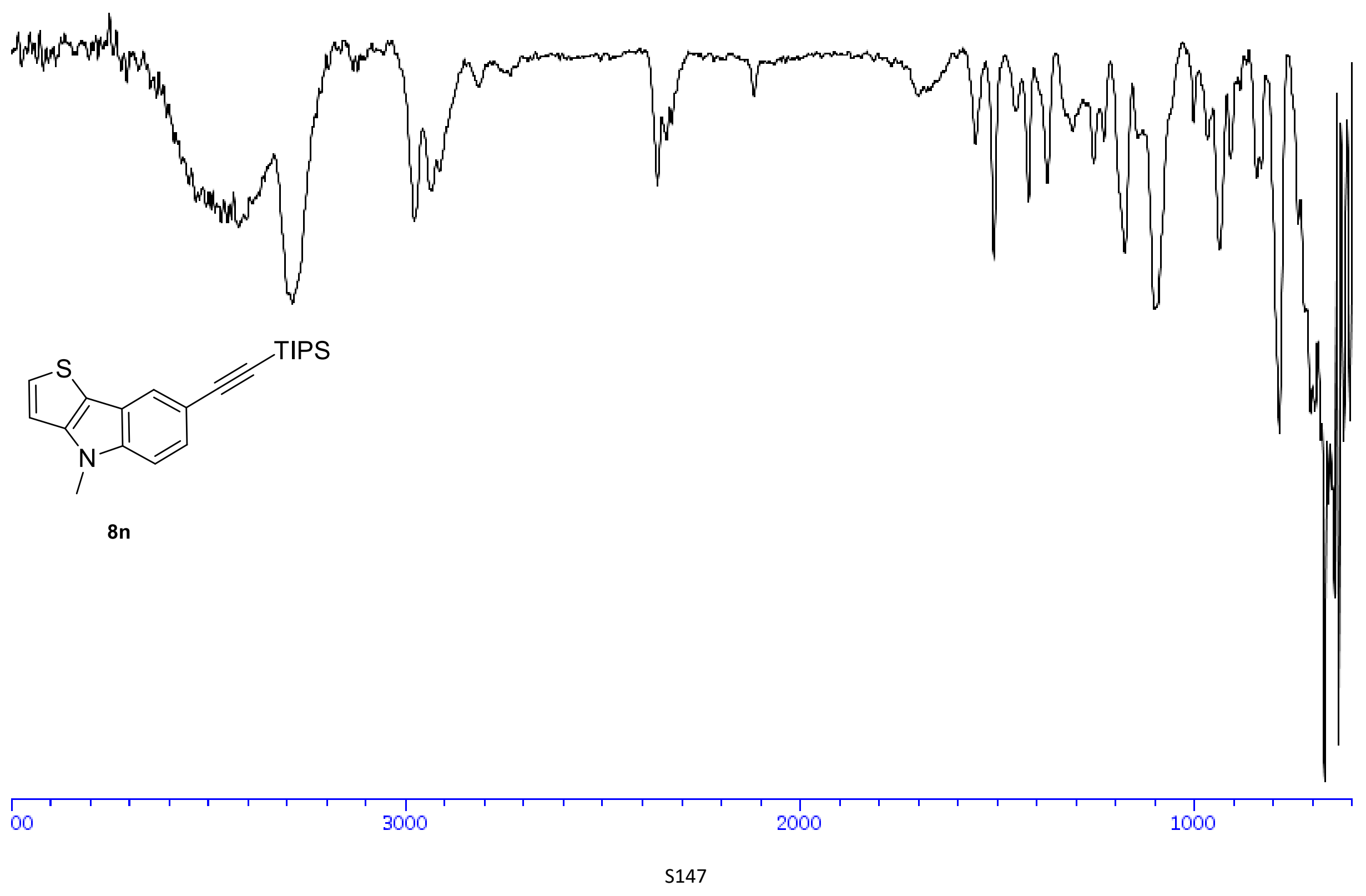


solvent: $\angle \mathrm{CDCI} 3\rangle$

Frequency. $400.13 \mathrm{MHz}$

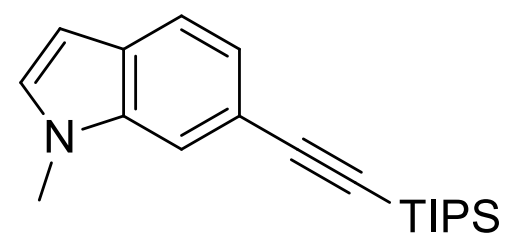

$10 a$
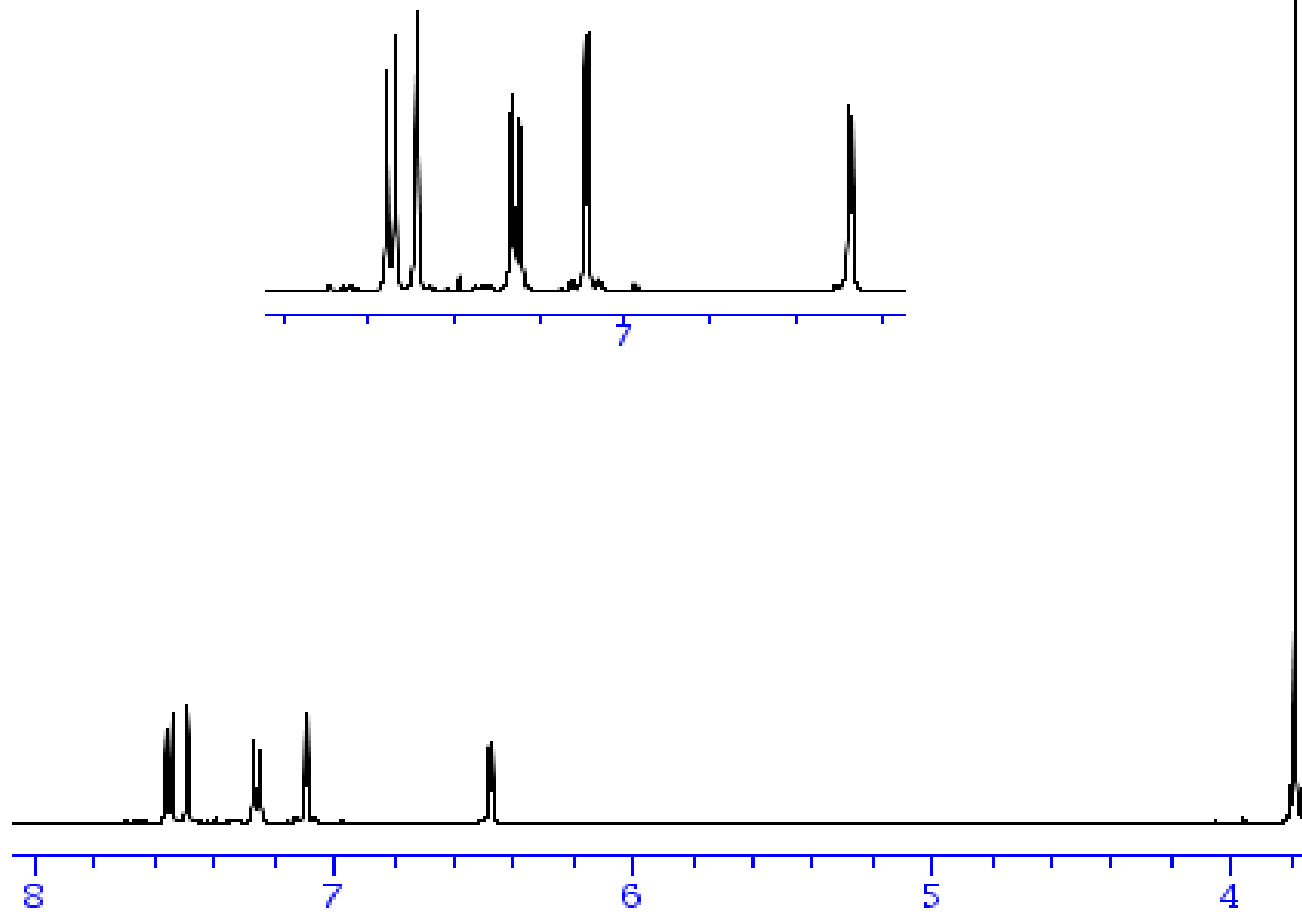
solvent: $<\mathrm{CDCI} 3>$

Frequency. $100.612769 \mathrm{MHz}$

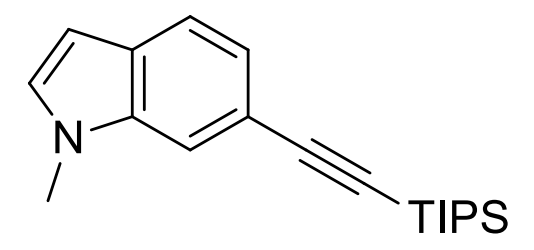

$10 a$

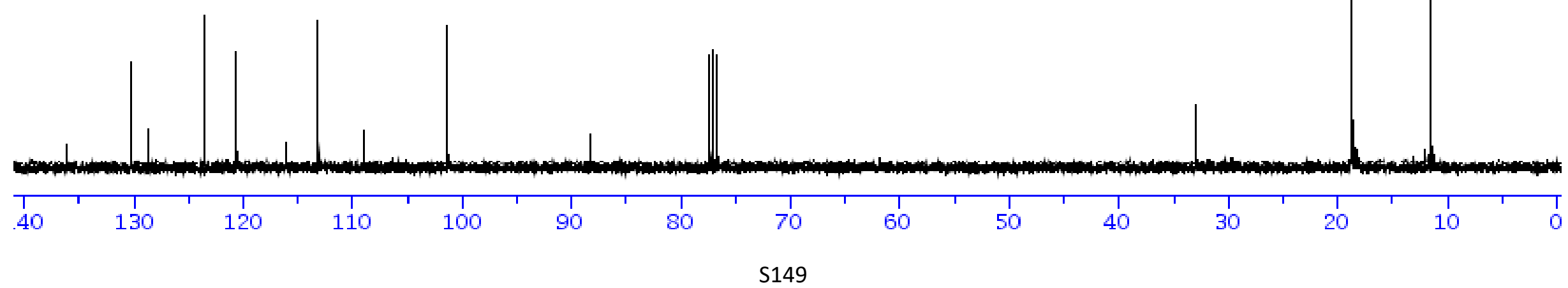




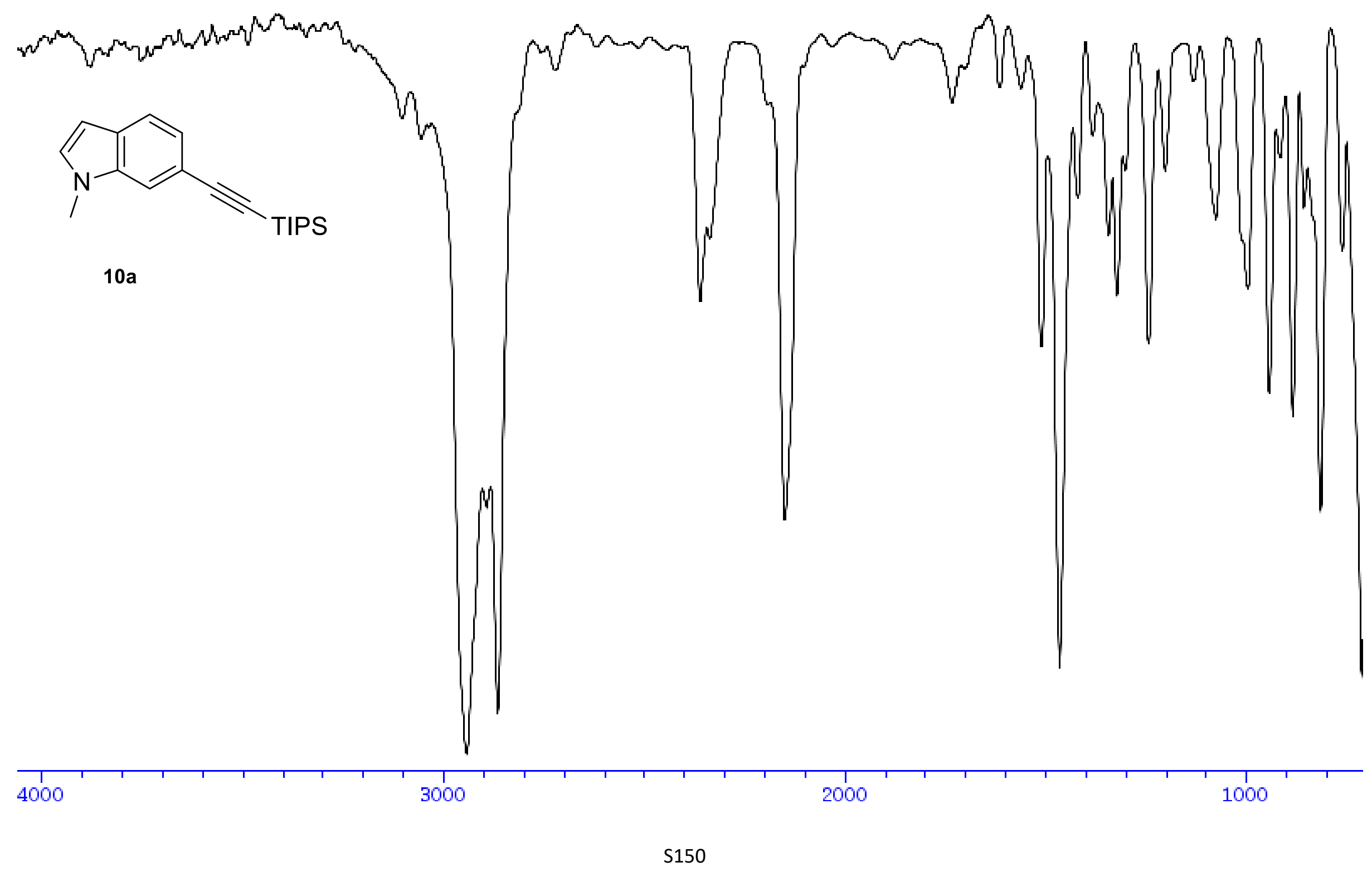


solvent: $<\mathrm{CDCI} 3>$

Frequency. $400.13 \mathrm{MHz}$

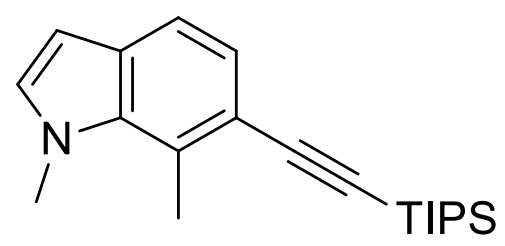

10b

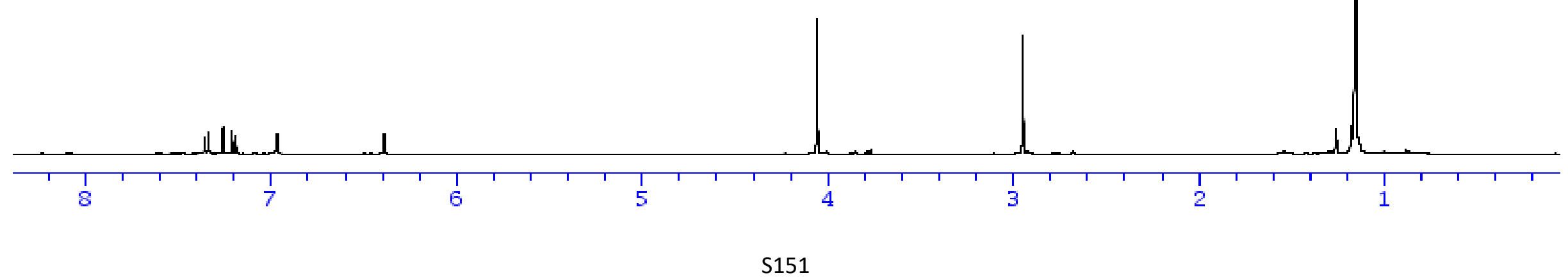


solvent: $\langle\mathrm{CDCl} 3\rangle$

Frequency. $100.612769 \mathrm{MHz}$

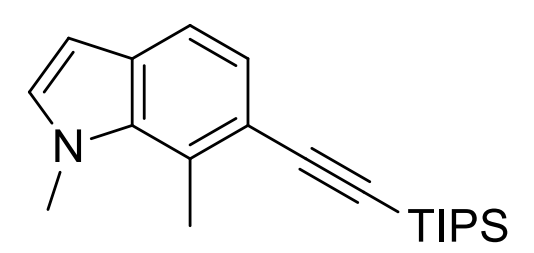

$10 \mathrm{~b}$
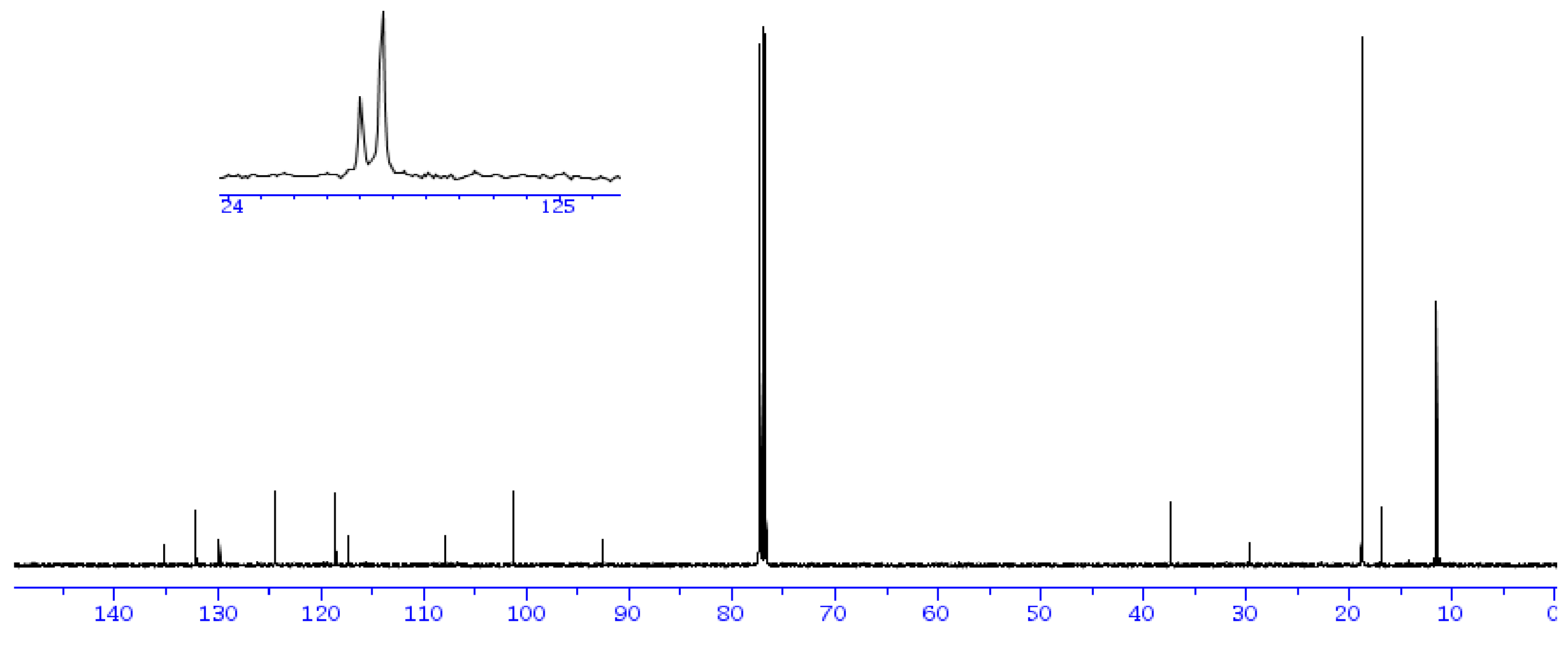


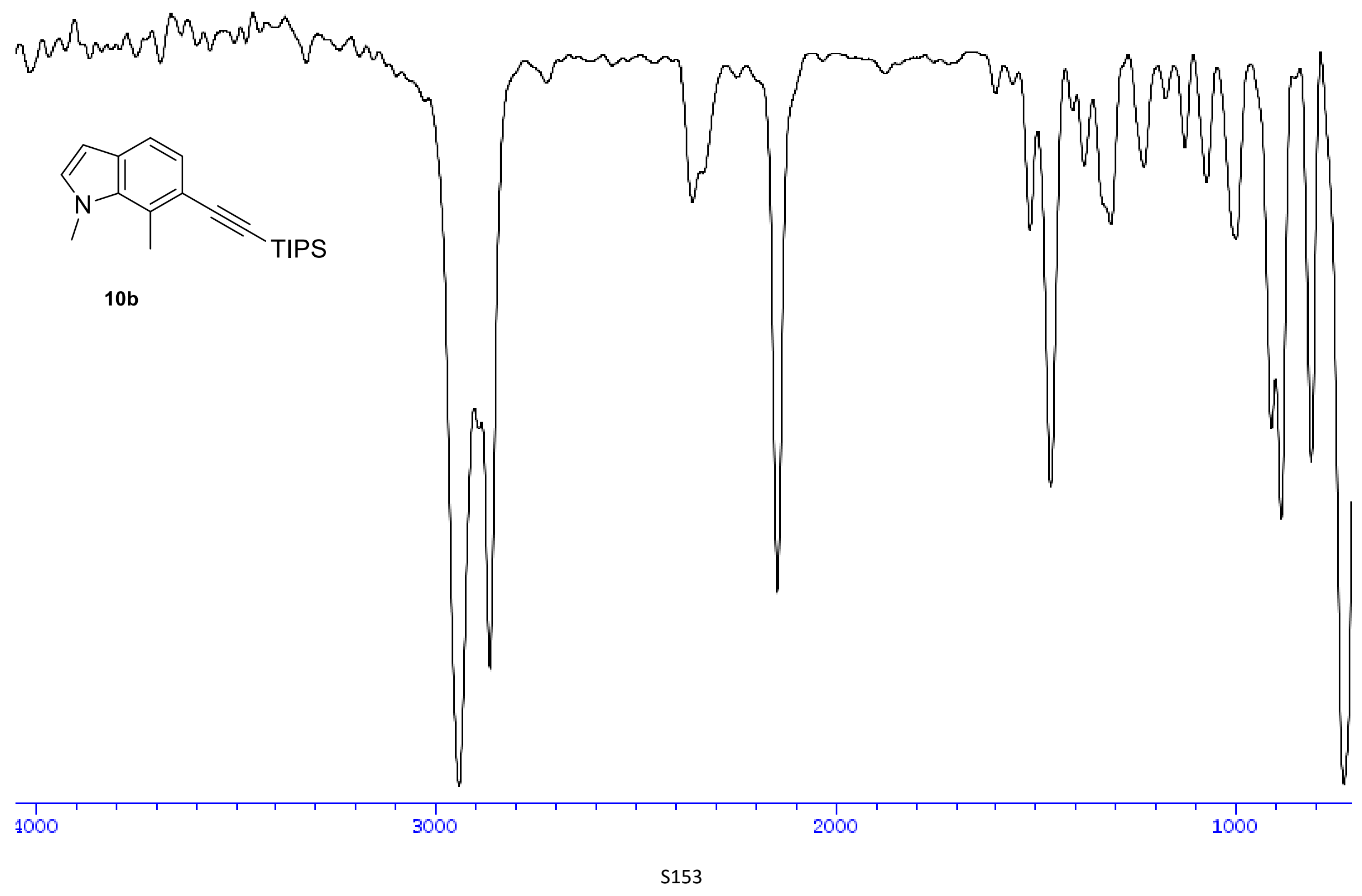


solvent: $\langle\mathrm{CDCl} 3>$

Frequency. $400.13 \mathrm{MHz}$

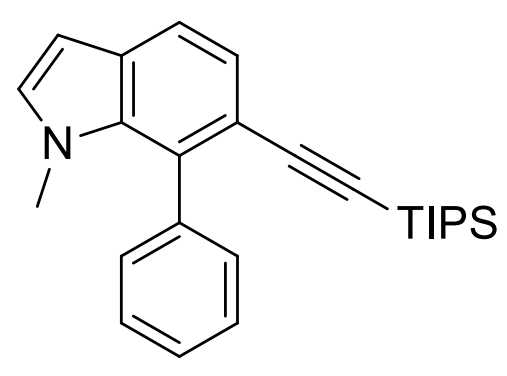

$10 c$

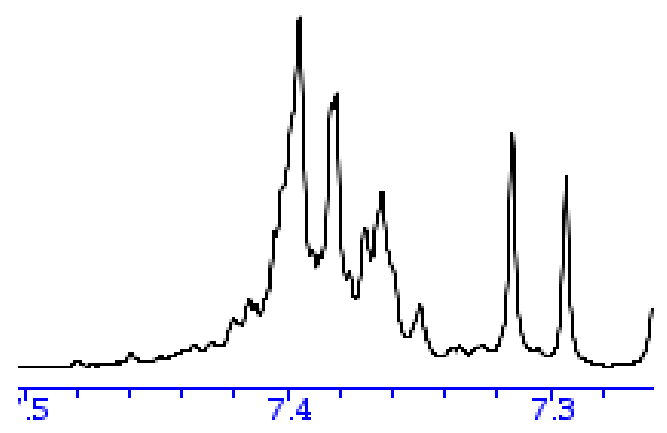

ate
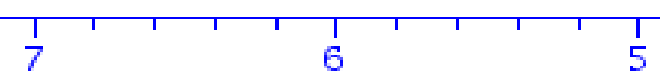

5 
solvent: $<\mathrm{CDCI} 3>$

Frequency. 100.612769MHz

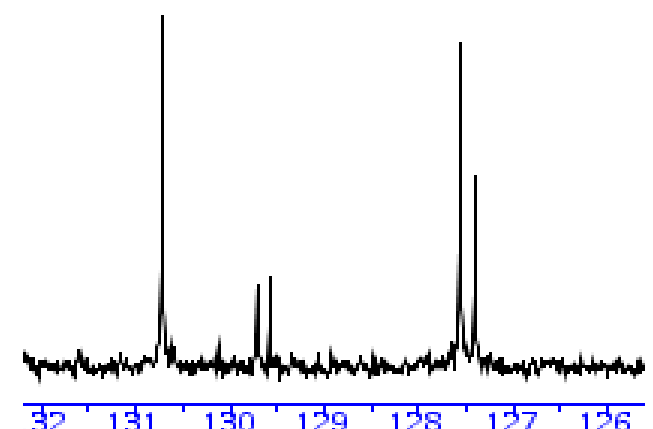

|| $\mid$ 


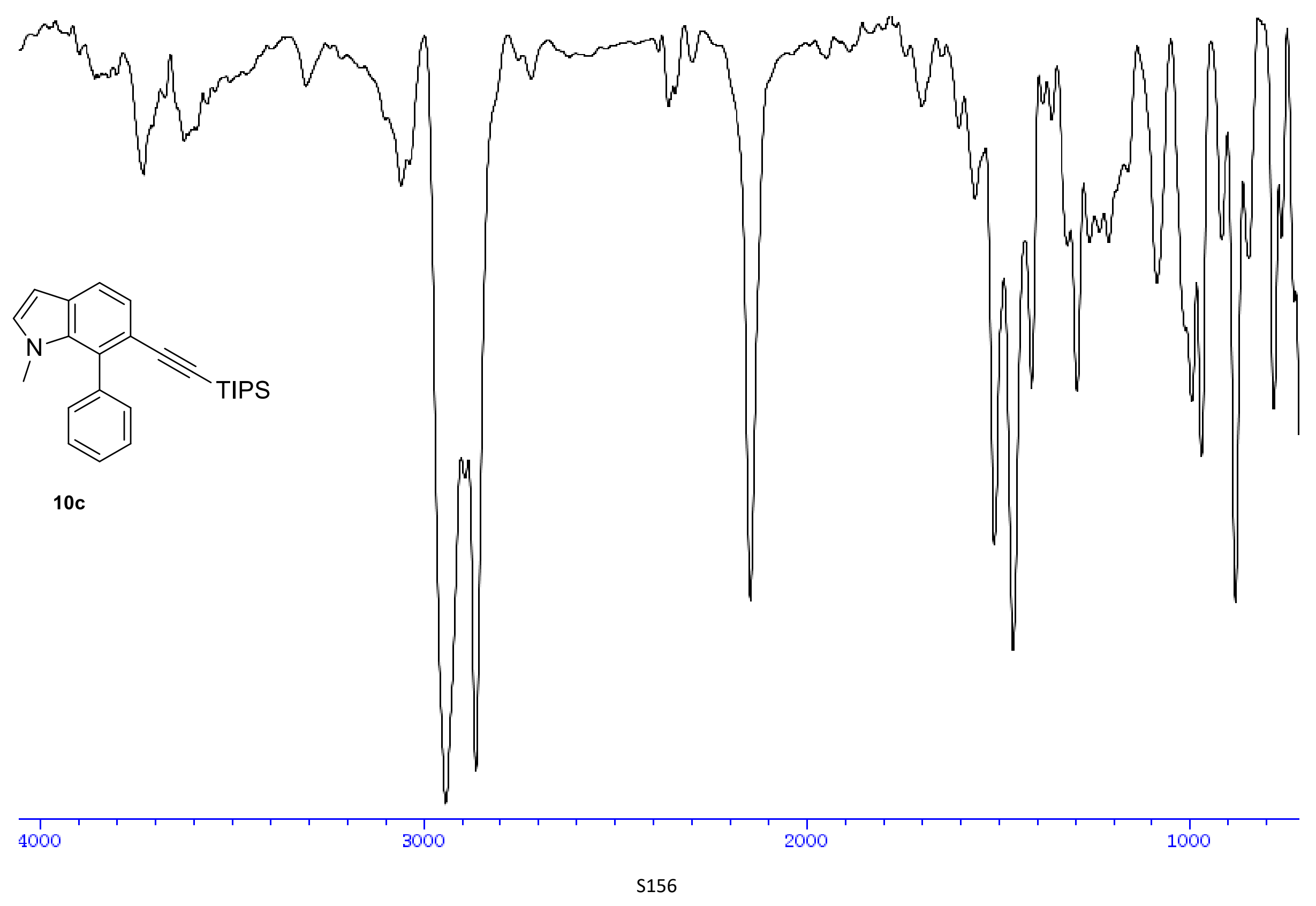


solvent: $\angle \mathrm{CDCl} 3>$

Frequency. 400.13MHz
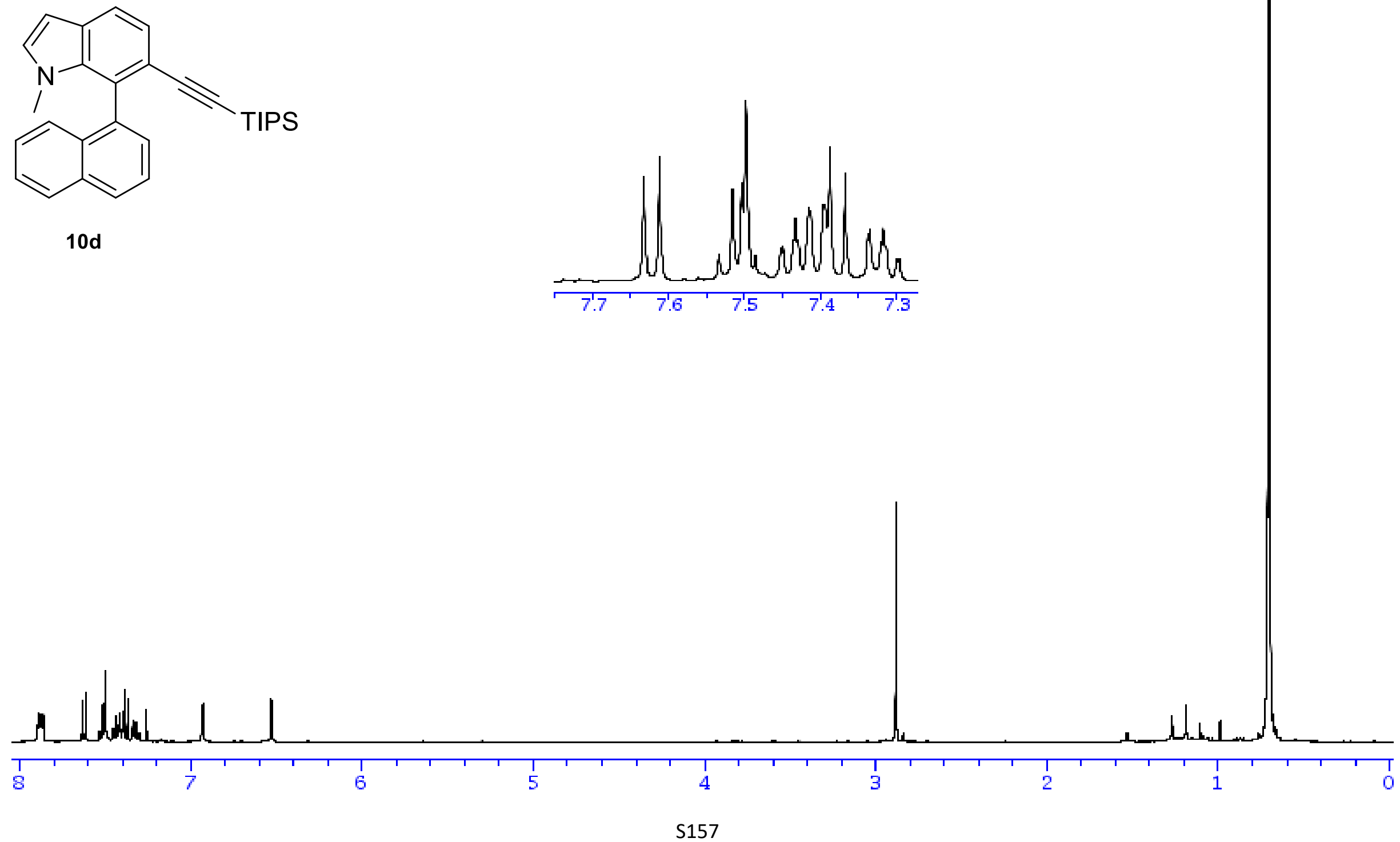
solvent: $<C D C I 3>$

Frequency. $100.612769 \mathrm{MHz}$
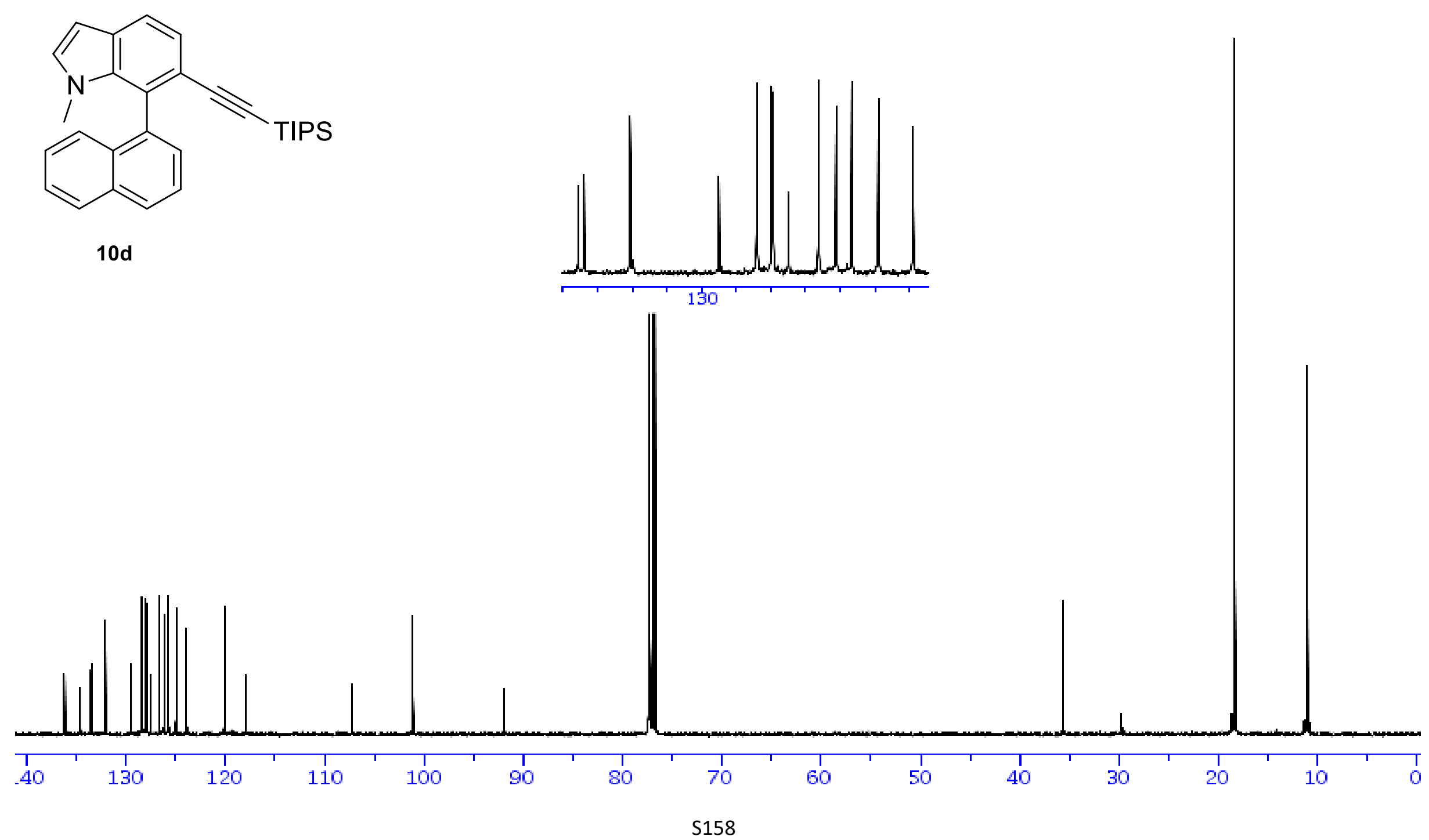


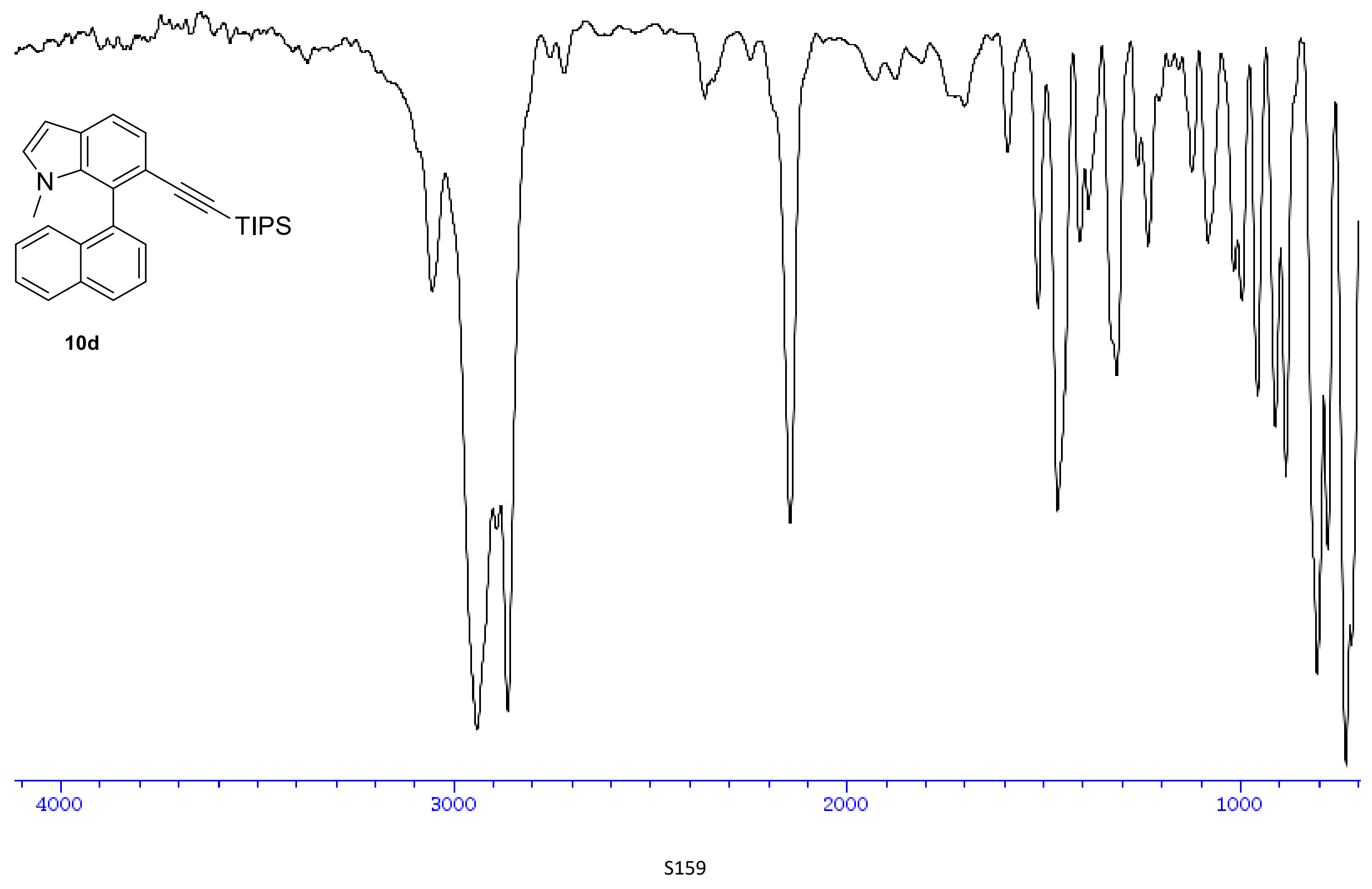


solvent: $\langle\mathrm{CDCI} 3\rangle$

Frequency. $400.13 \mathrm{MHz}$

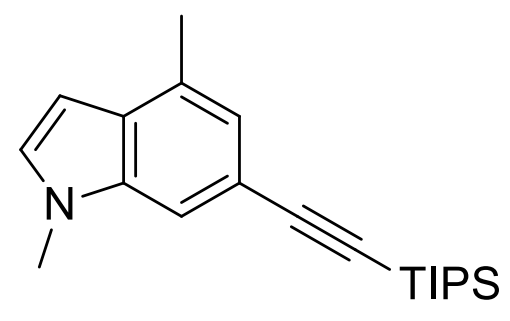

$10 \mathrm{e}$

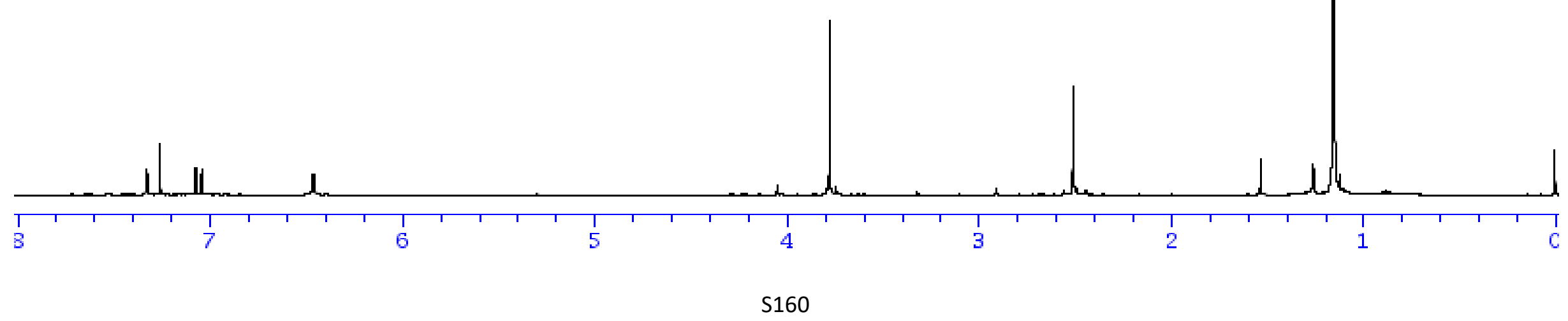


solvent: $<\mathrm{CDCI}>$

Frequency. $100.612769 \mathrm{MHz}$

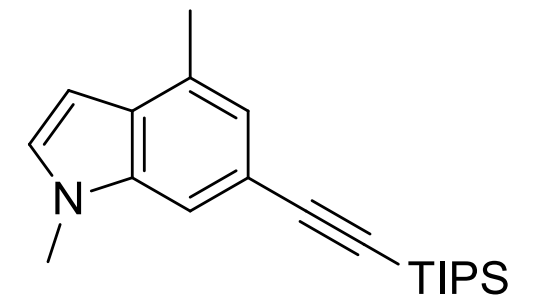

$10 e$
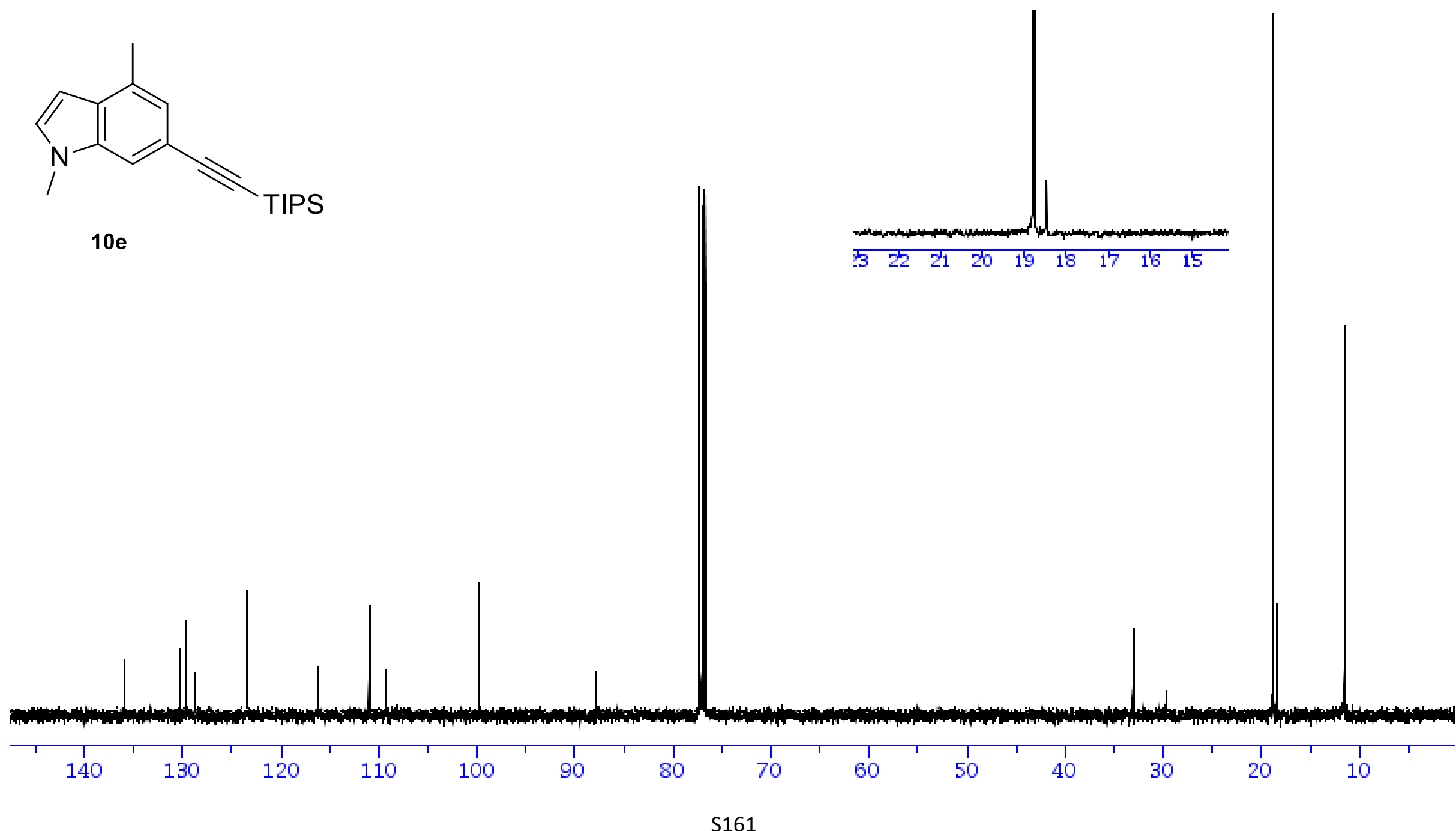


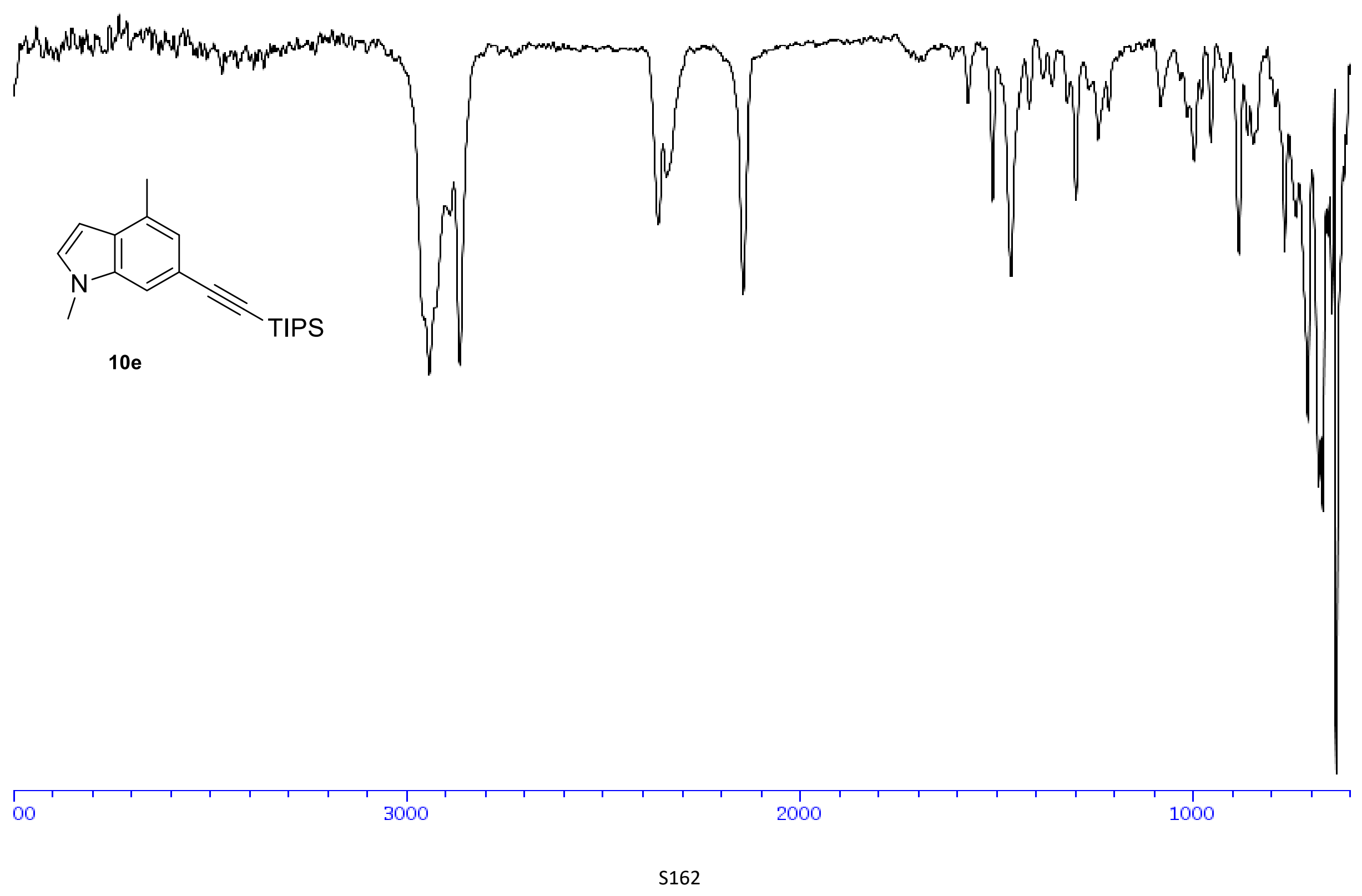


solvent: $<\mathrm{CDCI} 3>$

Frequency. $400.13 \mathrm{MHz}$
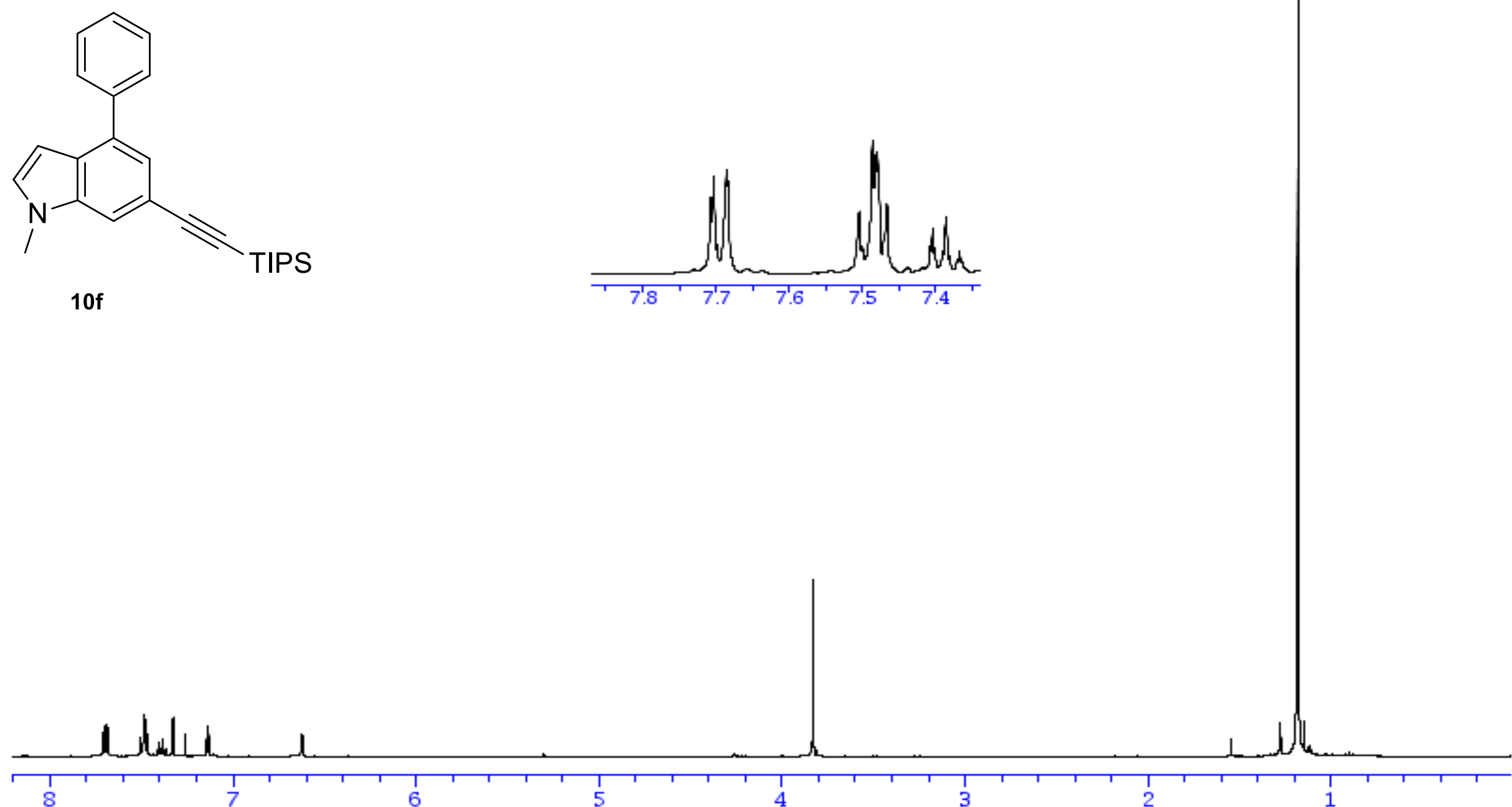
solvent: $\langle\mathrm{CDCl} 3>$

Frequency. 100.612769M Hz
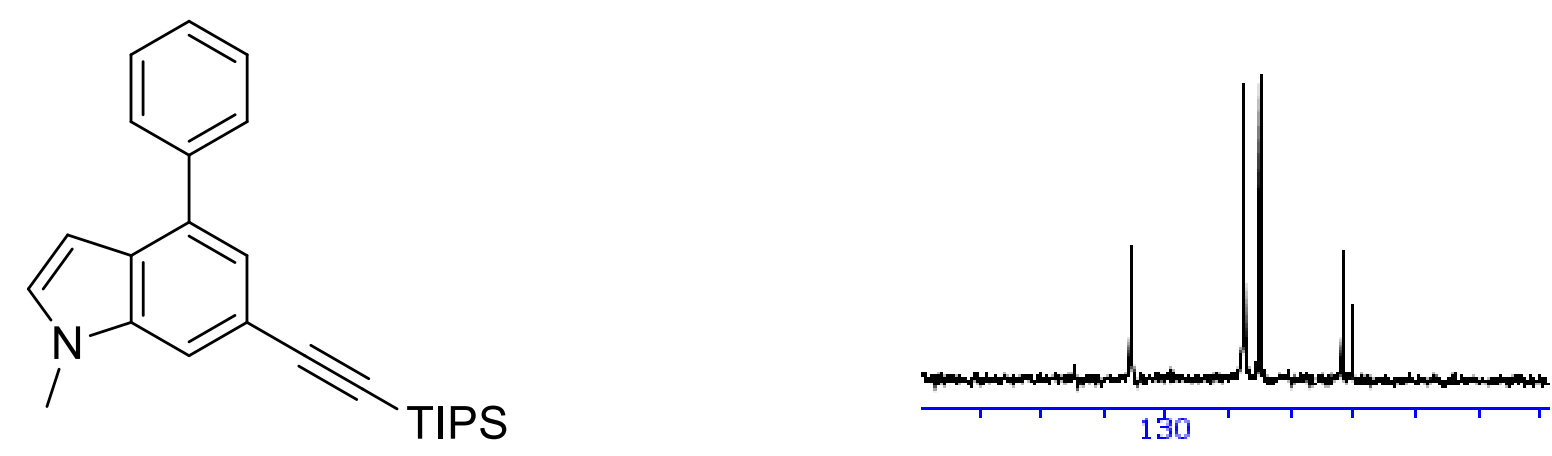

$10 f$

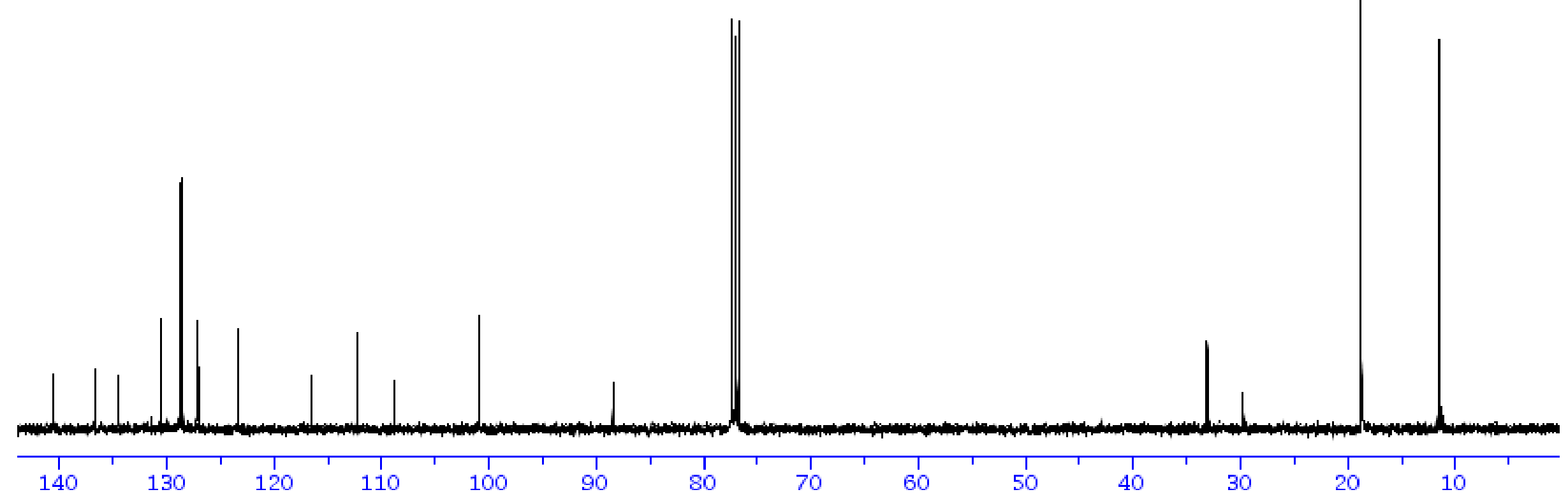




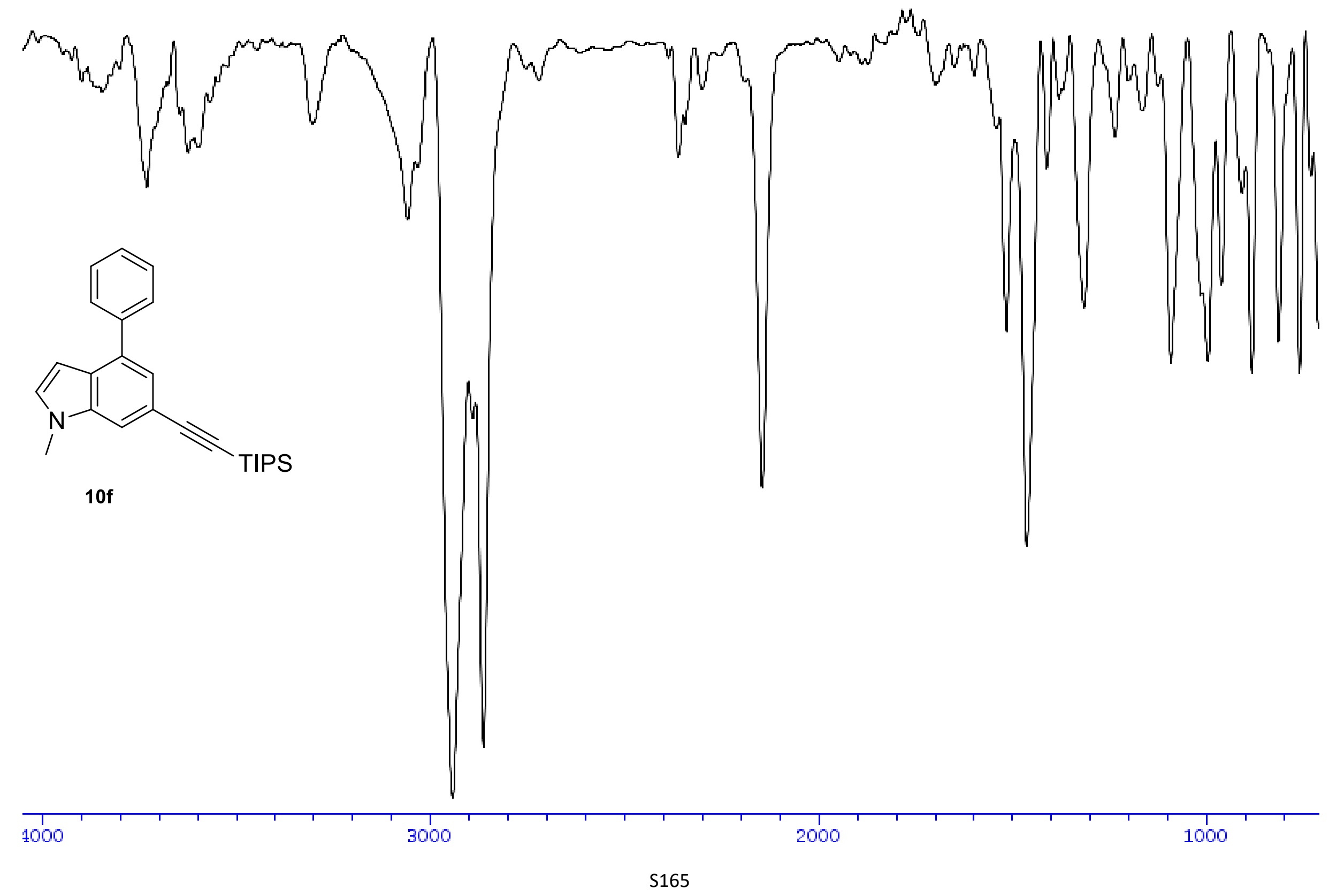


solvent: $<\mathrm{CDCI} 3>$

Frequency. $400.13 \mathrm{MHz}$

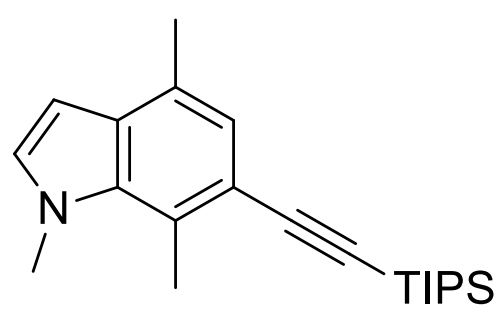

$10 \mathrm{~g}$

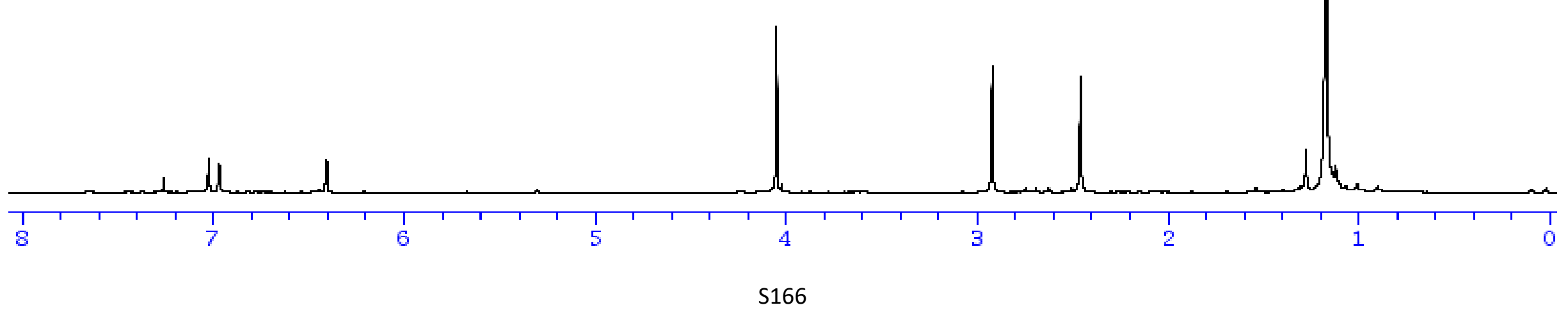


solvent: $\langle\mathrm{CDCl} 3>$

Frequency. $100.612769 \mathrm{MHz}$
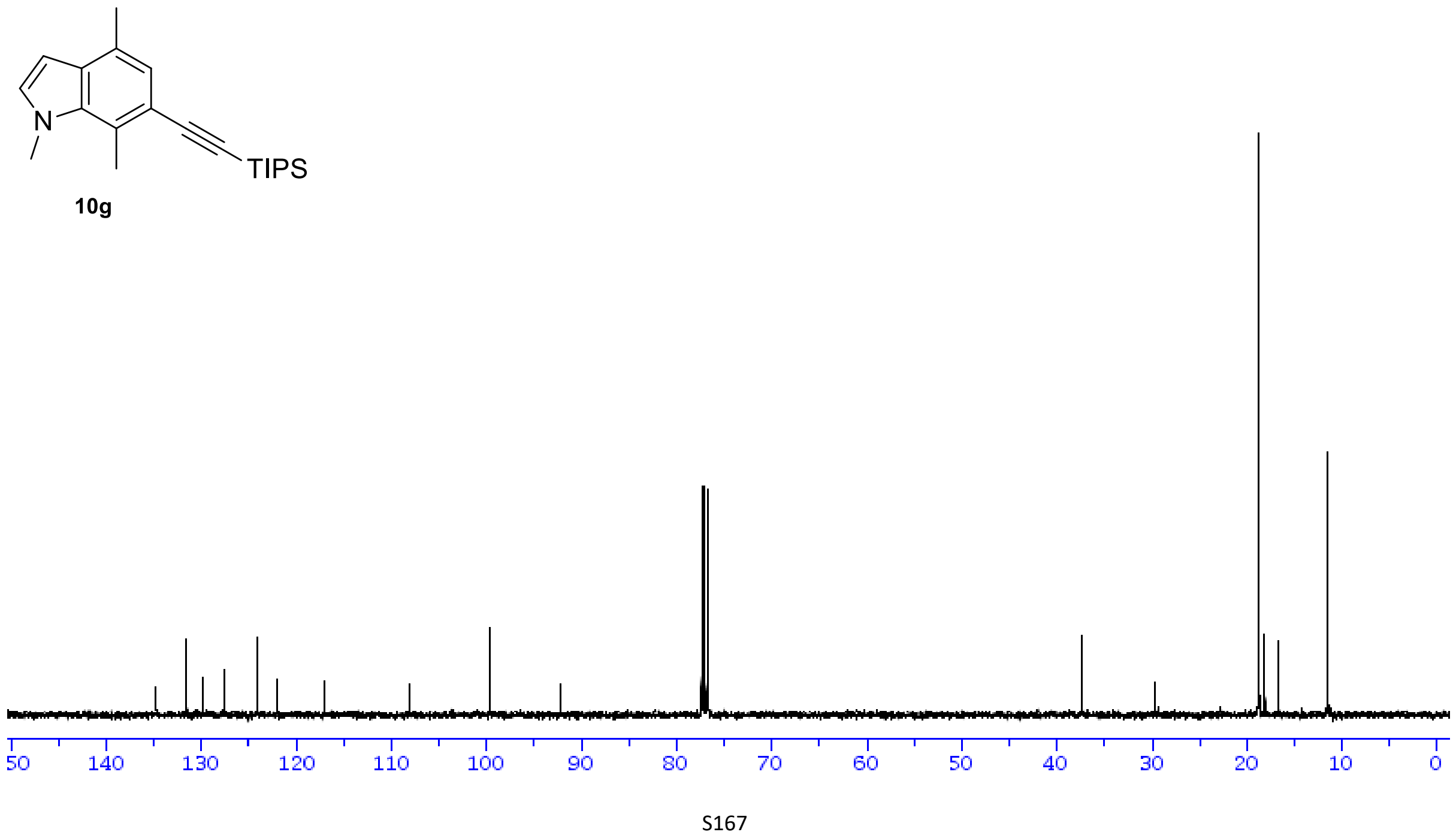


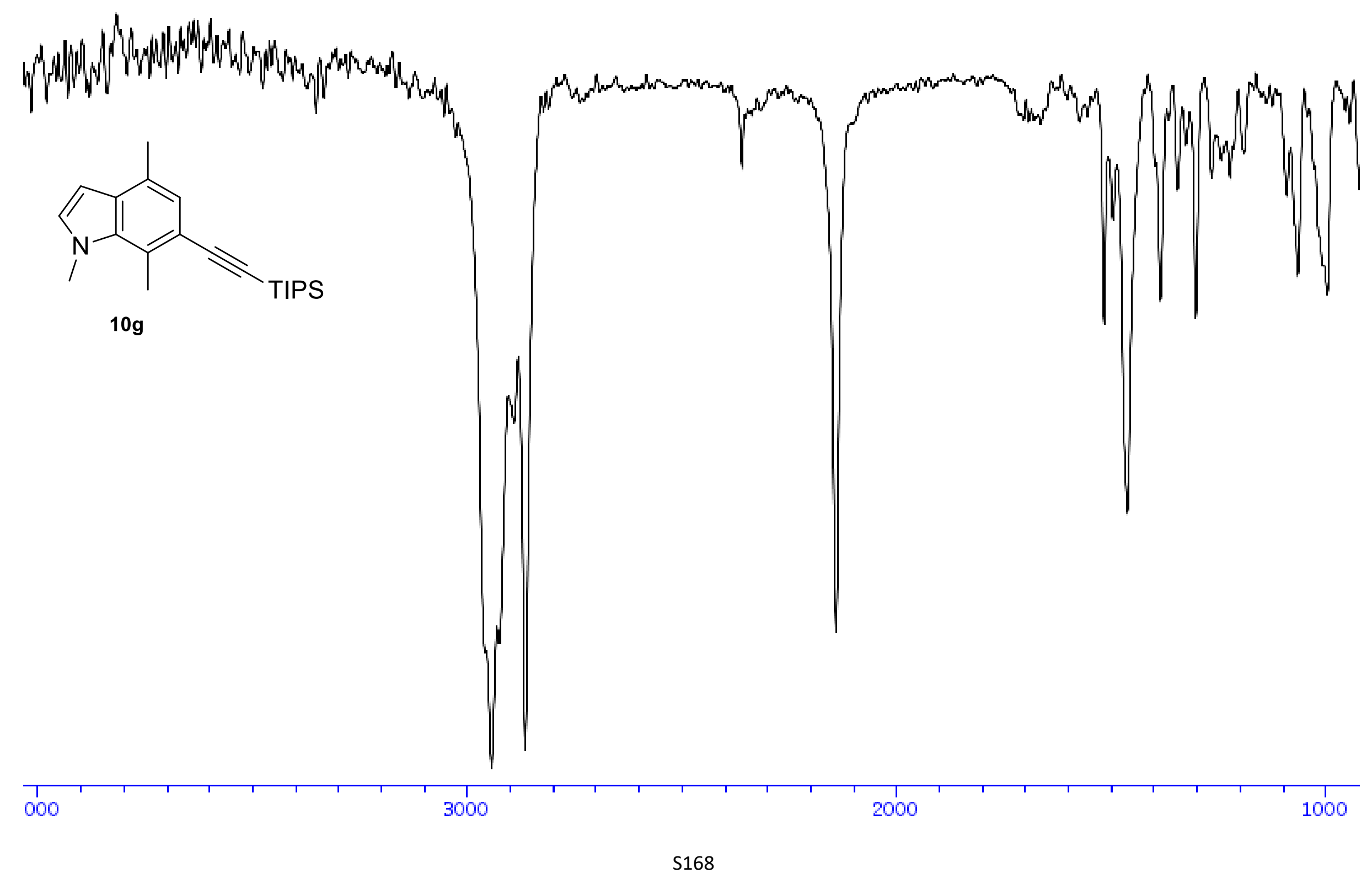


solvent: $\langle\mathrm{CDCI} 3\rangle$

Frequency. $400.13 \mathrm{MHz}$

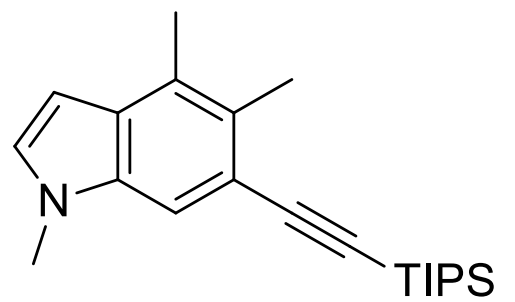

$10 \mathrm{~h}$

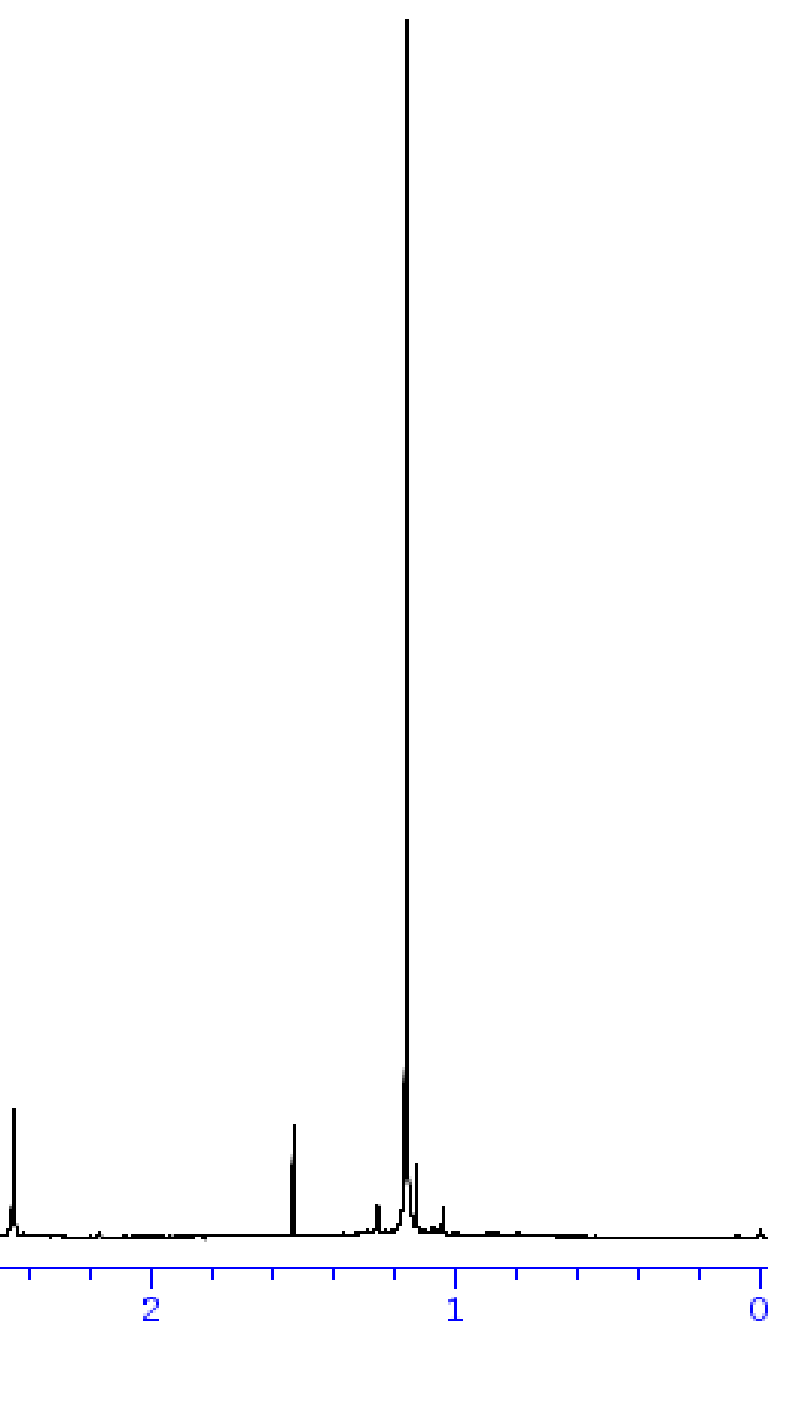


solvent: $\langle\mathrm{CDCI} 3>$

Frequency. 201. 192849M Hz
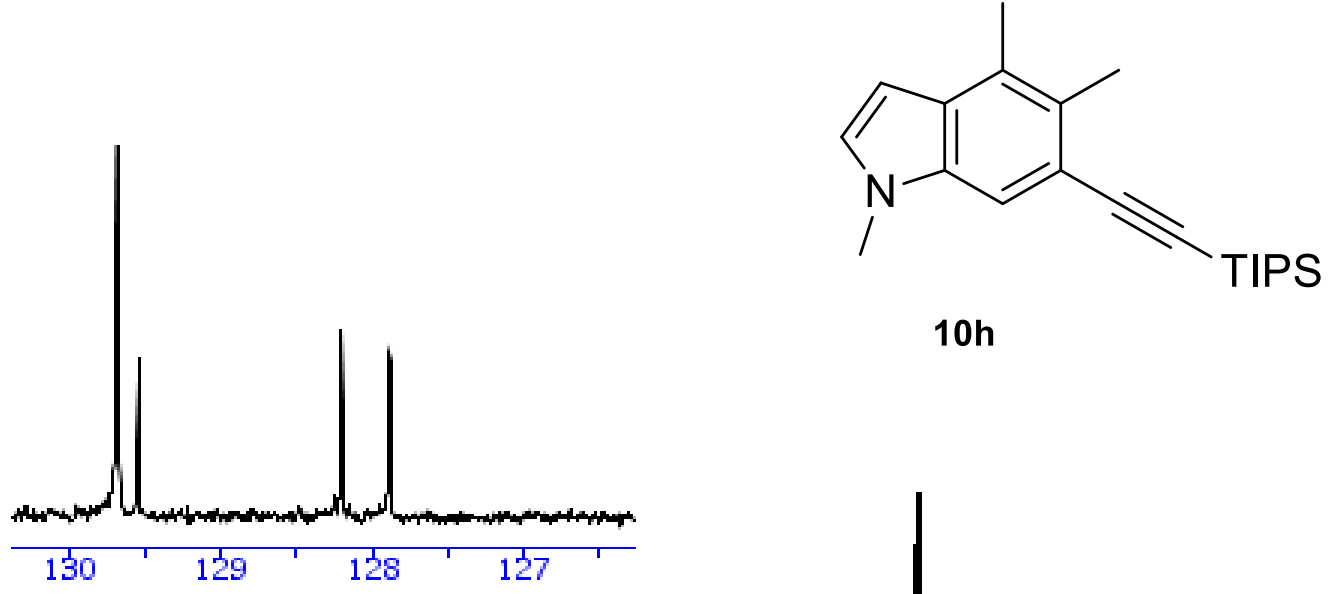

$10 \mathrm{~h}$

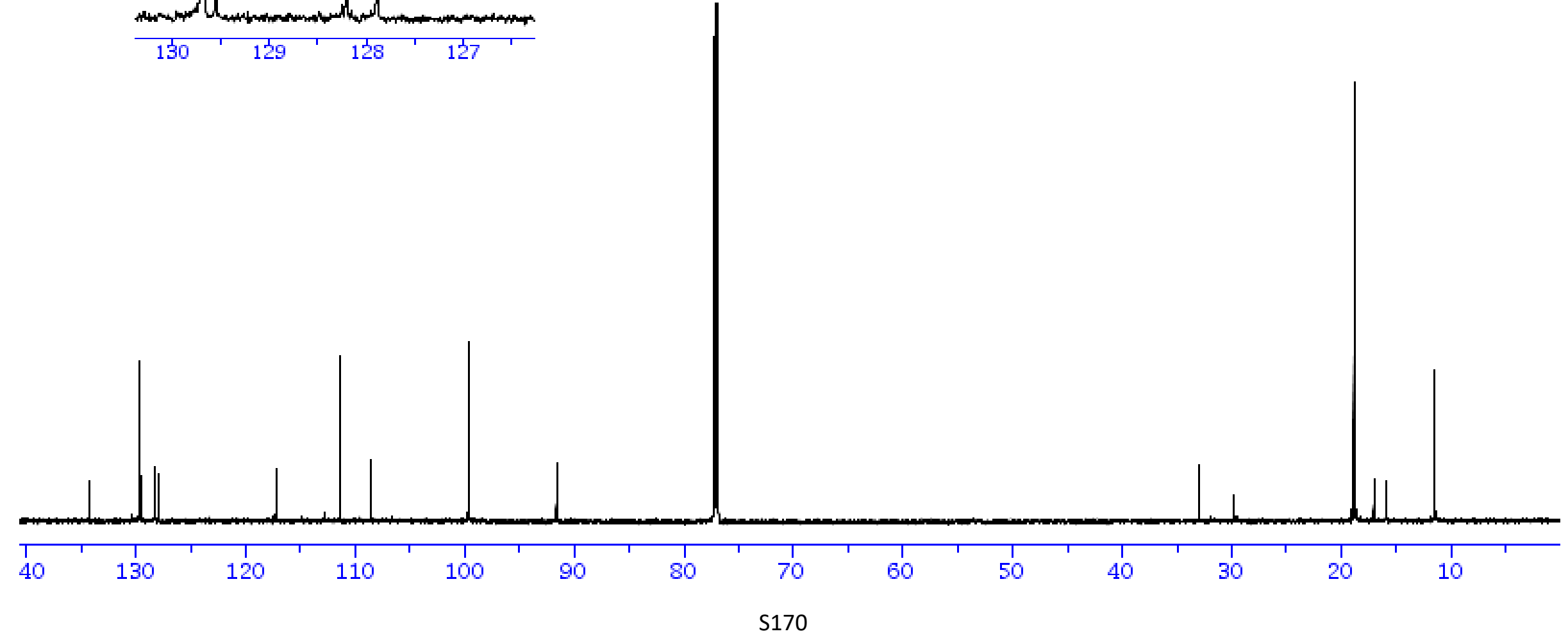




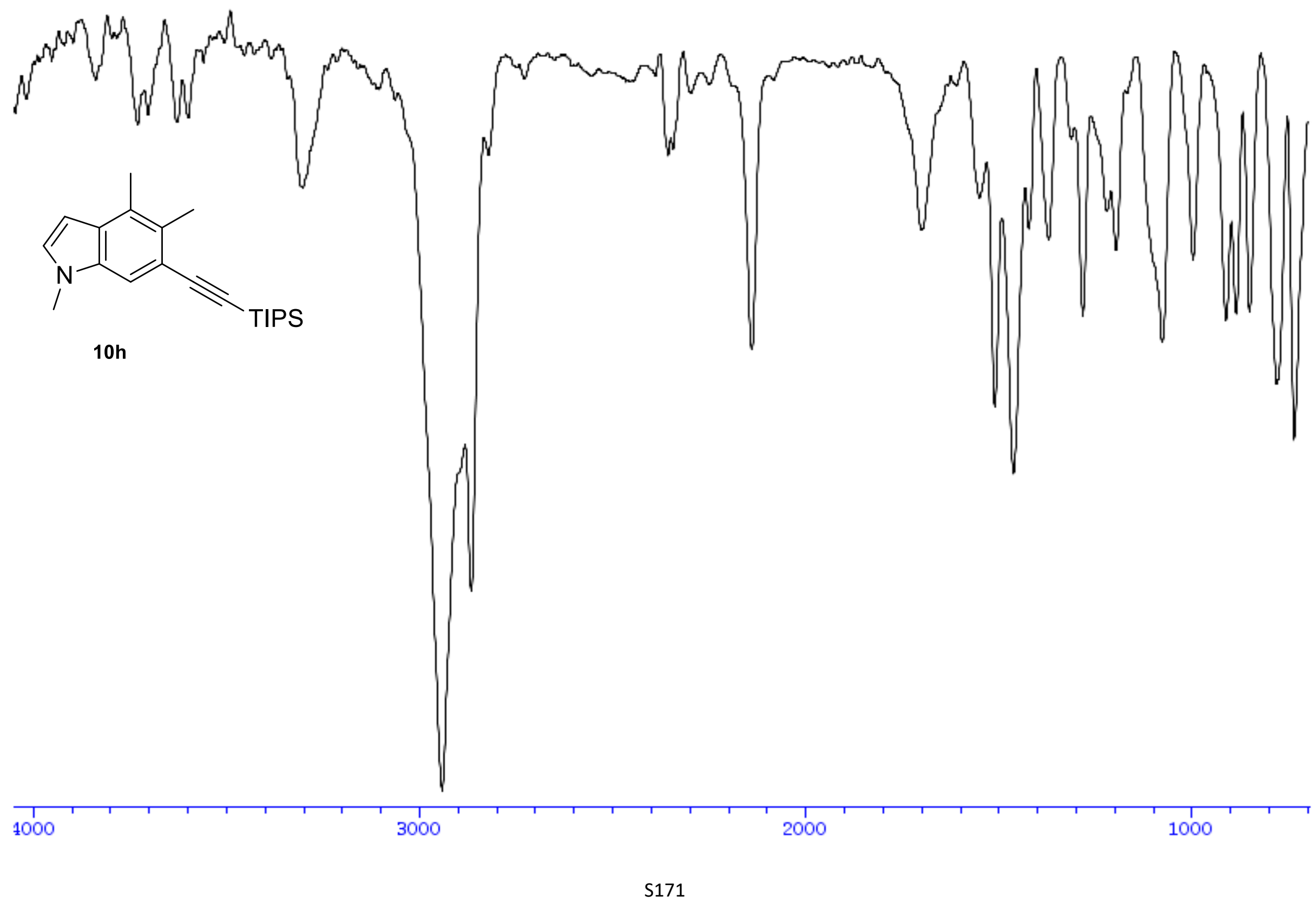


solvent: $\langle\mathrm{CDCI} 3>$

Frequency. $400.13 \mathrm{MHz}$
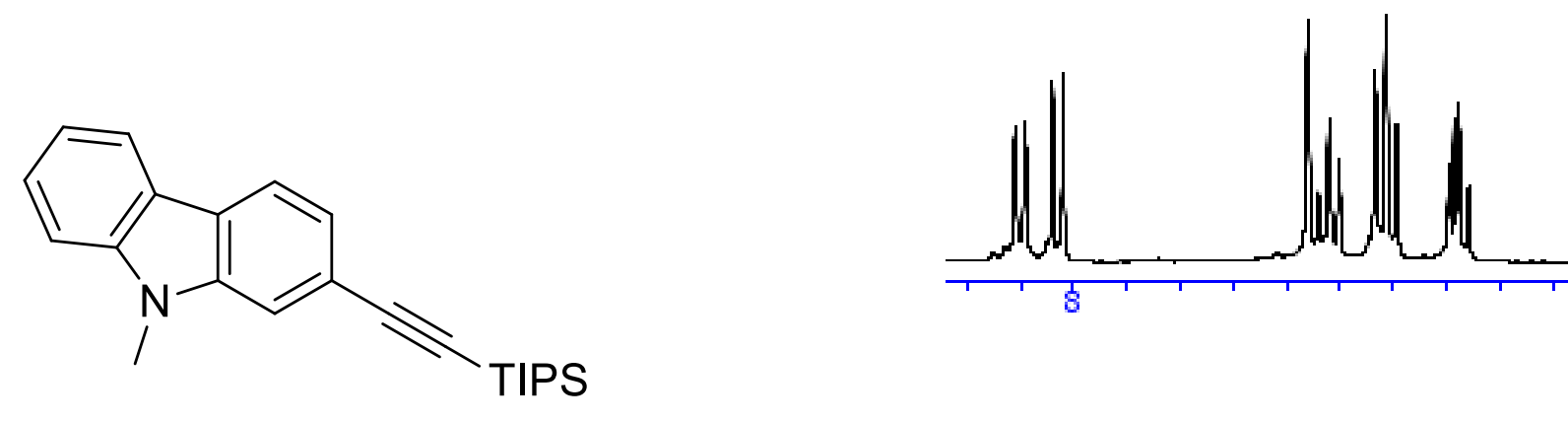

$10 \mathrm{i}$

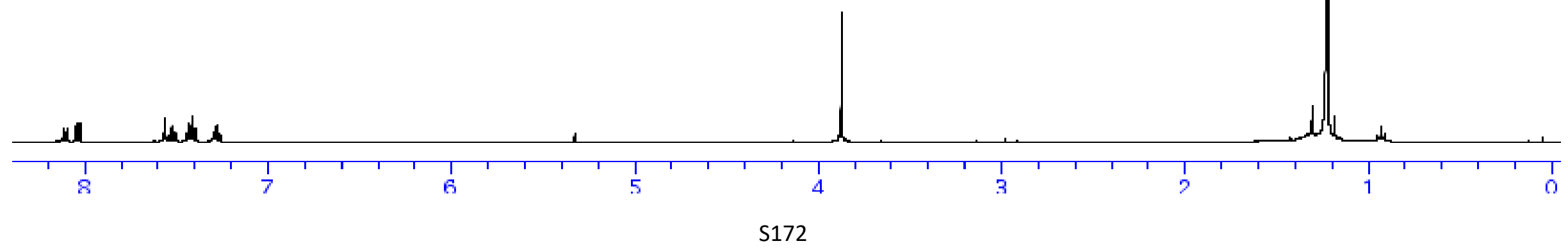


solvent: $\langle\mathrm{CDCI}>$

Frequency. $100.612769 \mathrm{MHz}$
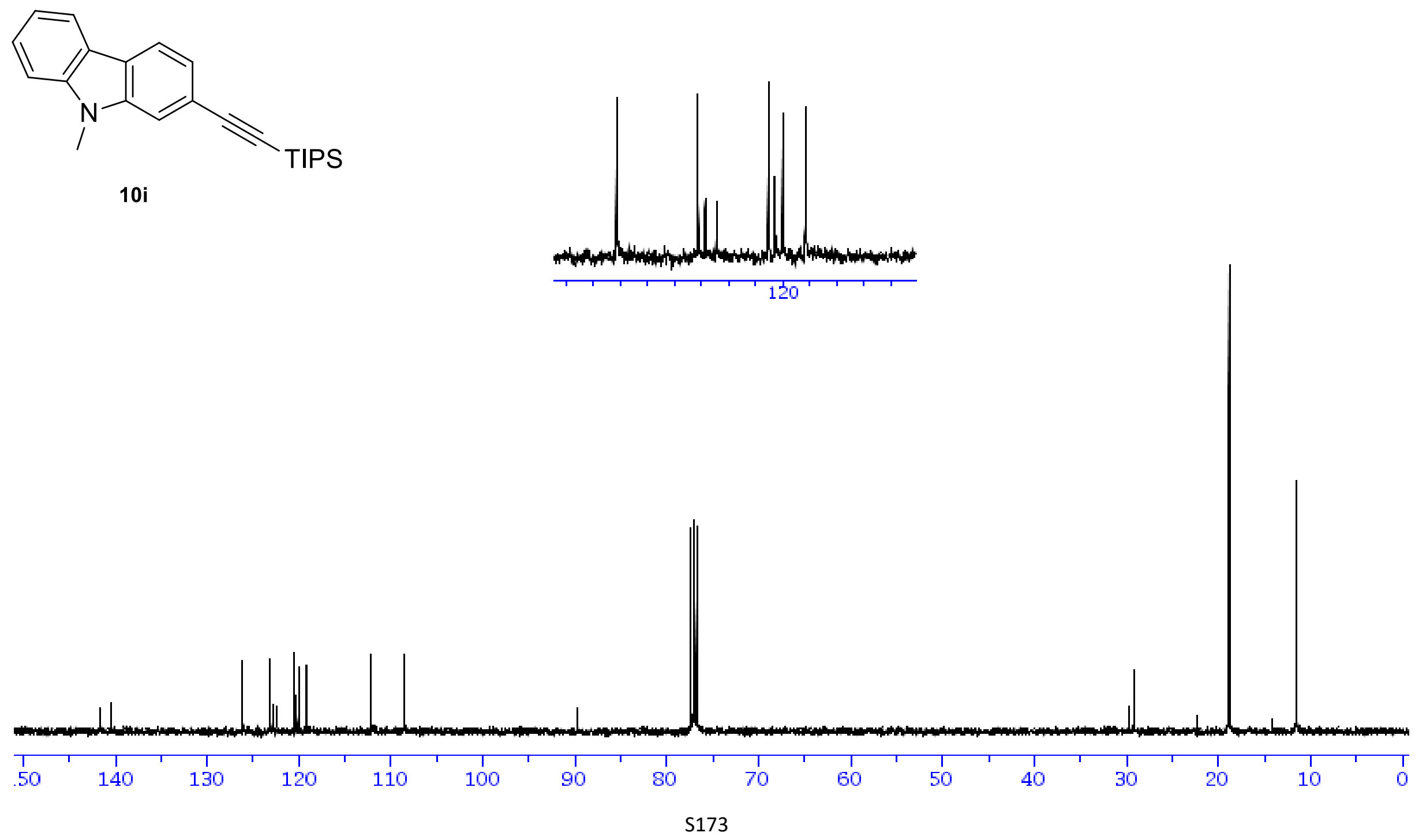


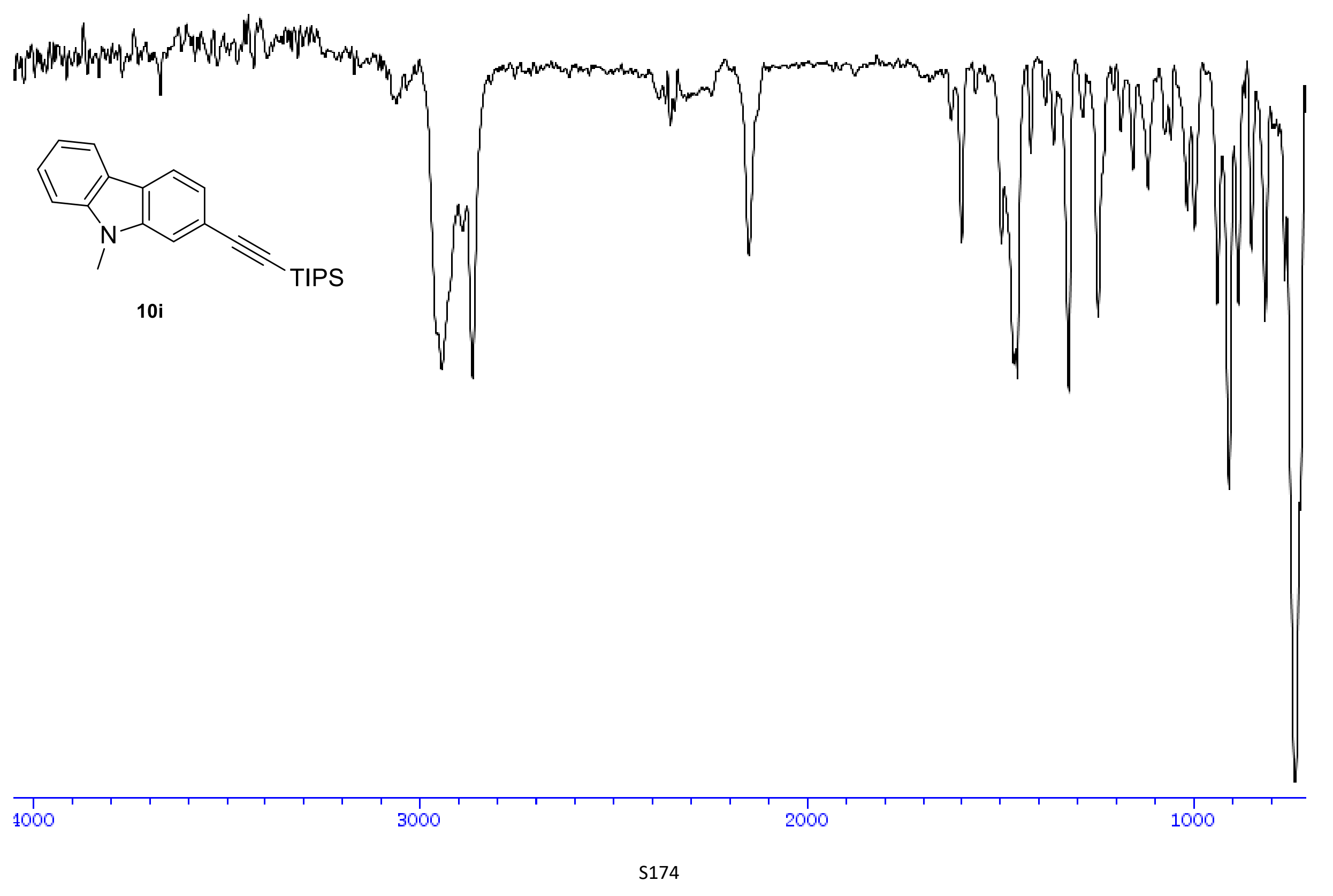


solvent: $\langle\mathrm{CDCl} 3>$

Frequency. $400.13 \mathrm{MHz}$

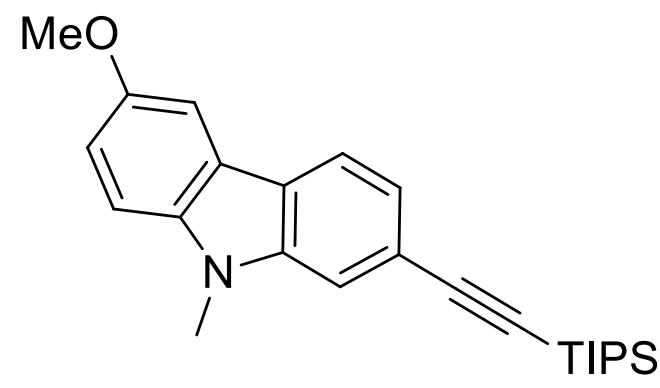

$10 \mathrm{j}$
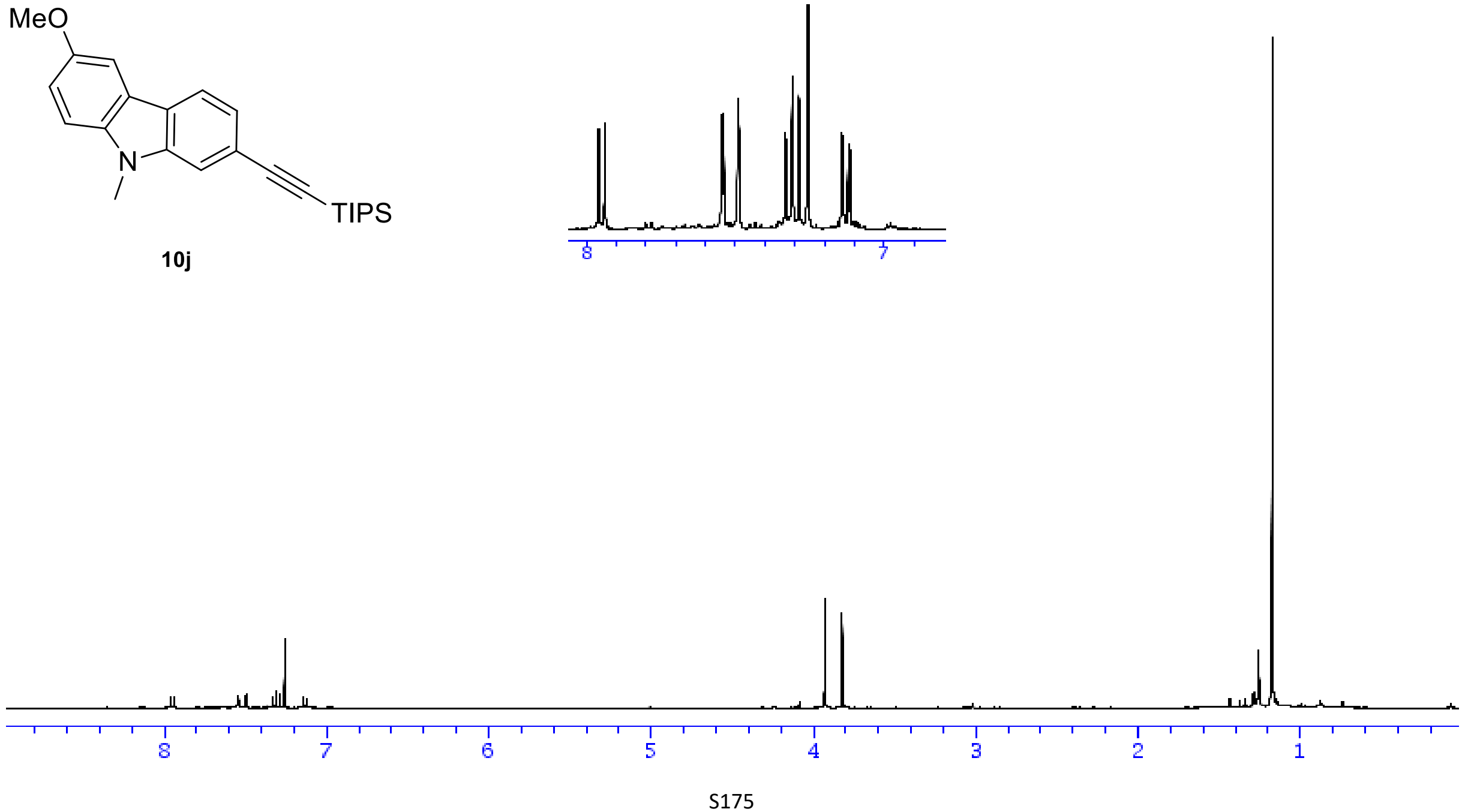


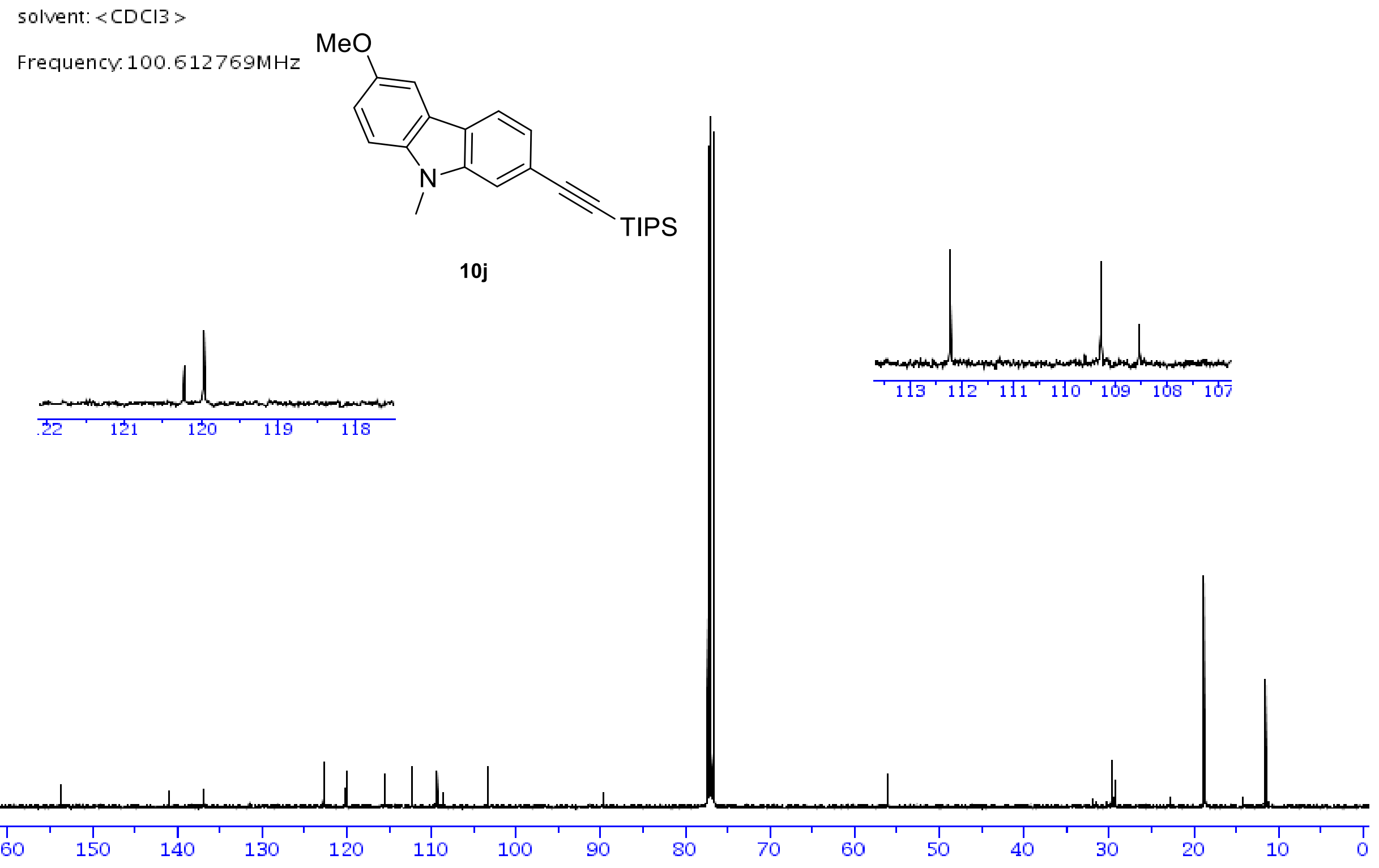




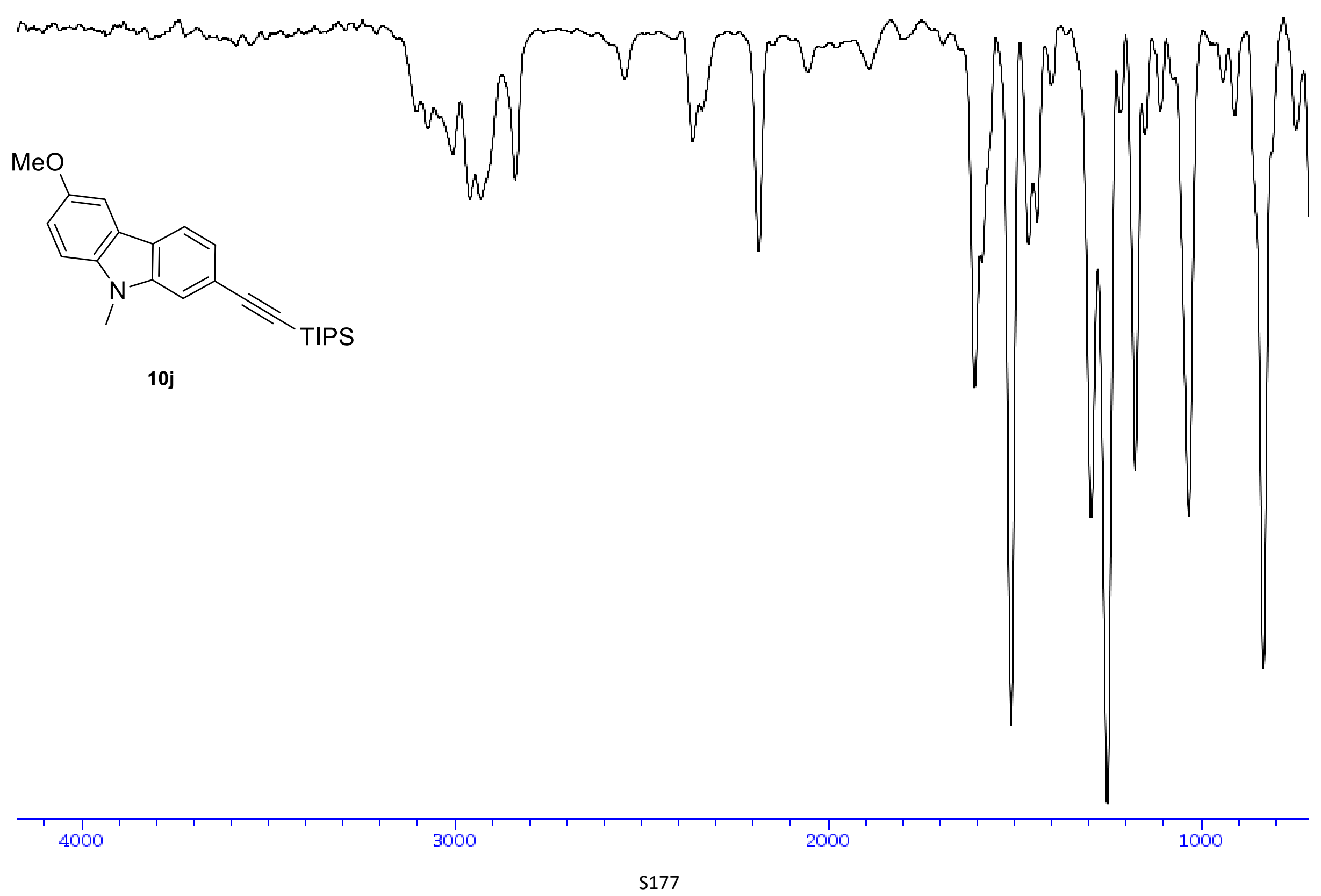


solvent: $\angle \mathrm{CDCl} 3\rangle$

Frequency. $400.13 \mathrm{MHz}$

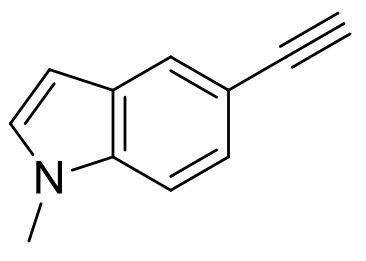

39

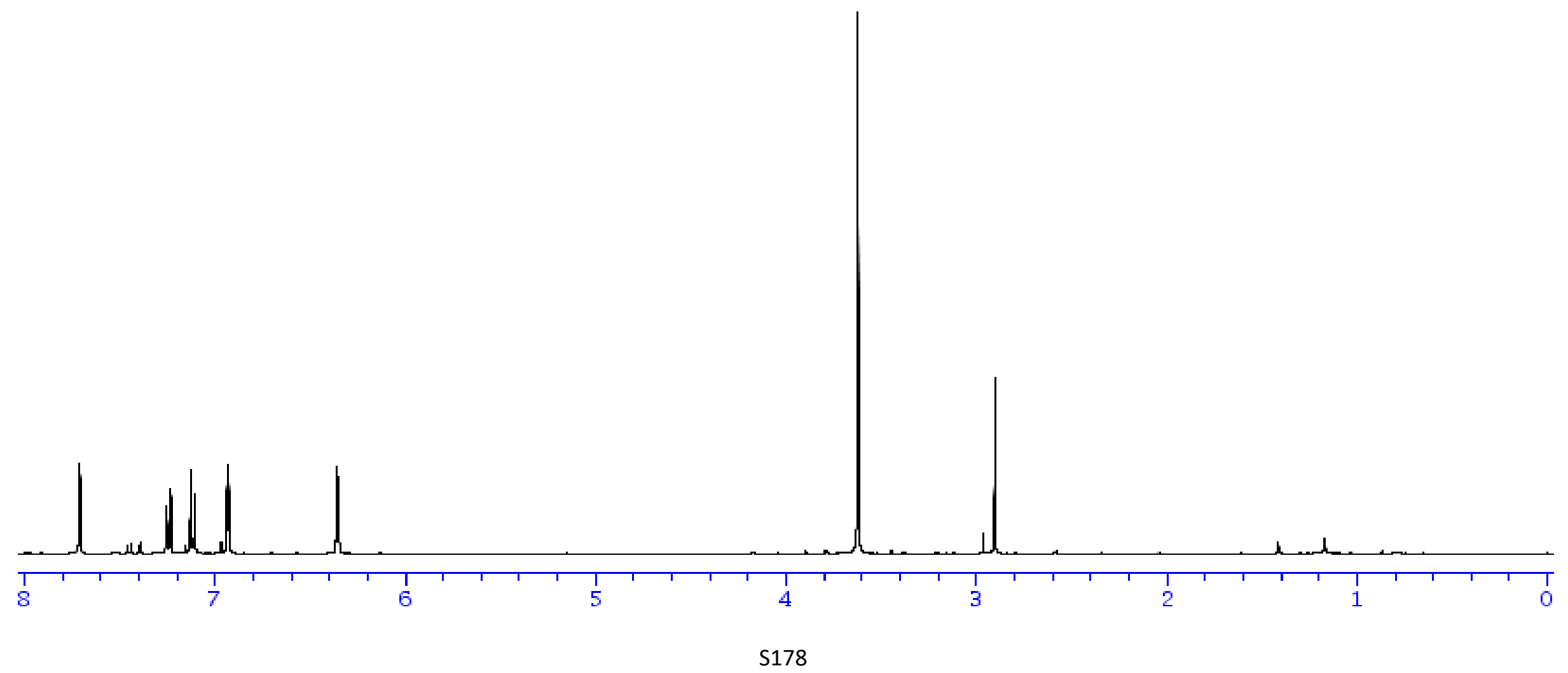


solvent: $\langle\mathrm{CDCI} 3>$

Frequency. $100.612769 \mathrm{MHz}$

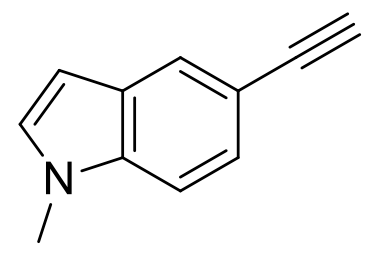

39
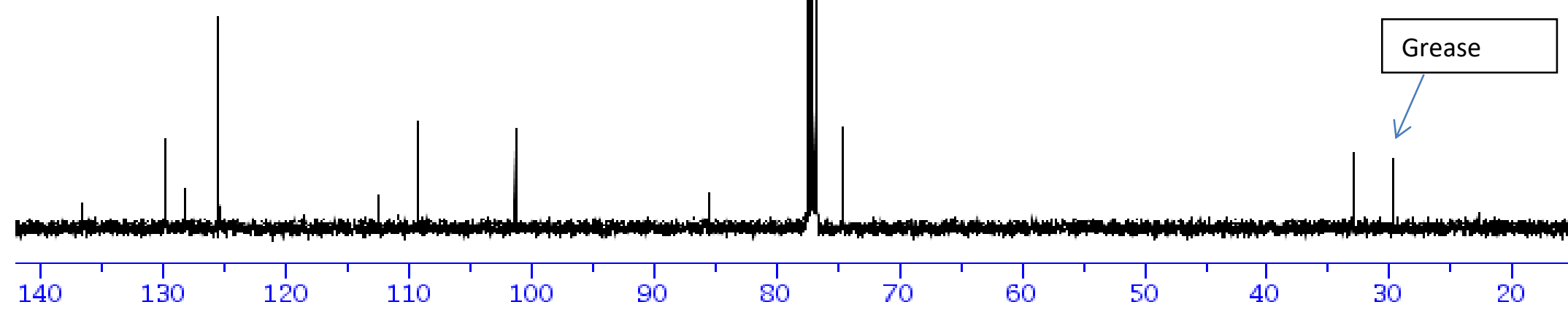

90 


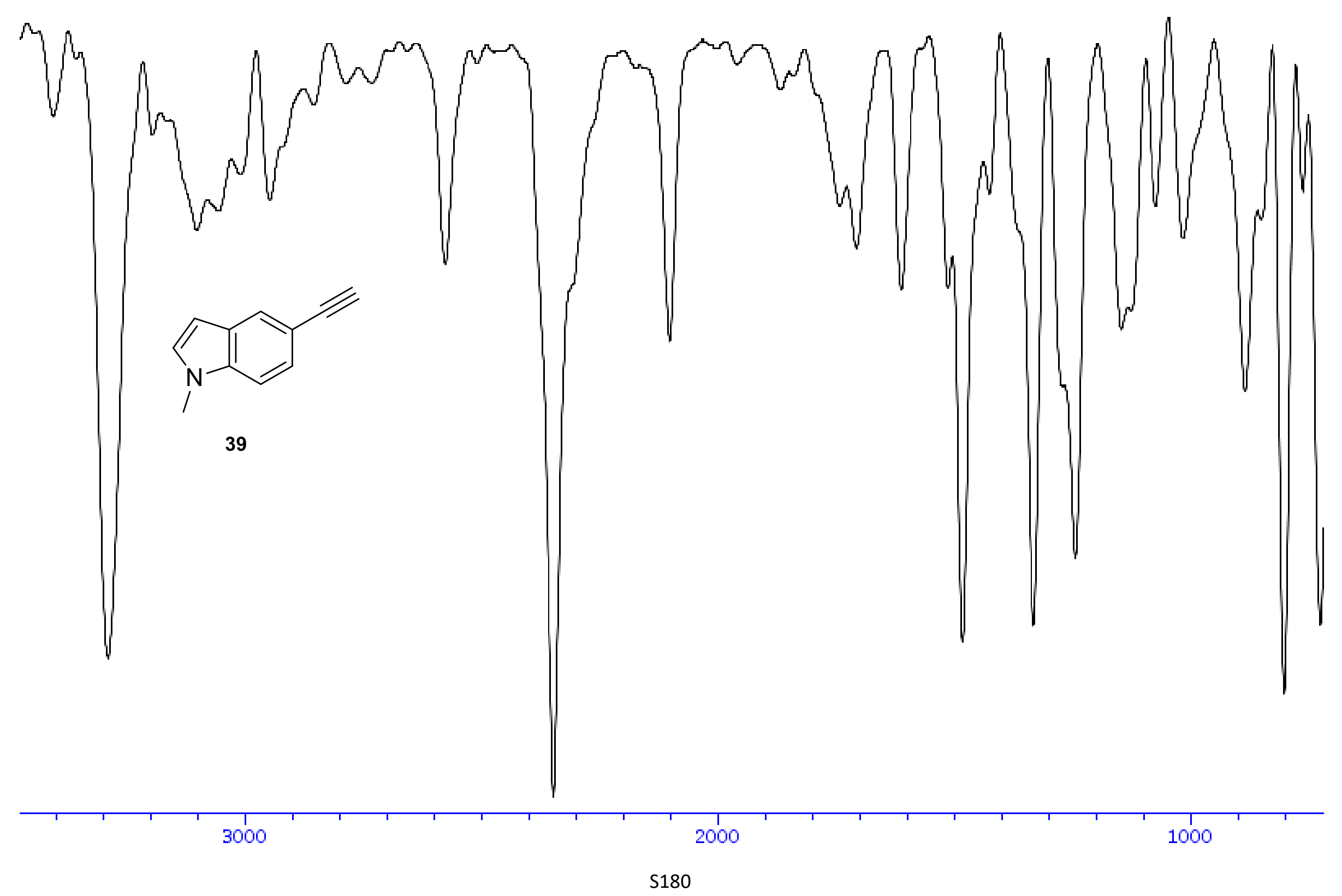


solvent: $\langle\mathrm{CDCI} 3>$

Frequency. $400.13 \mathrm{MHz}$
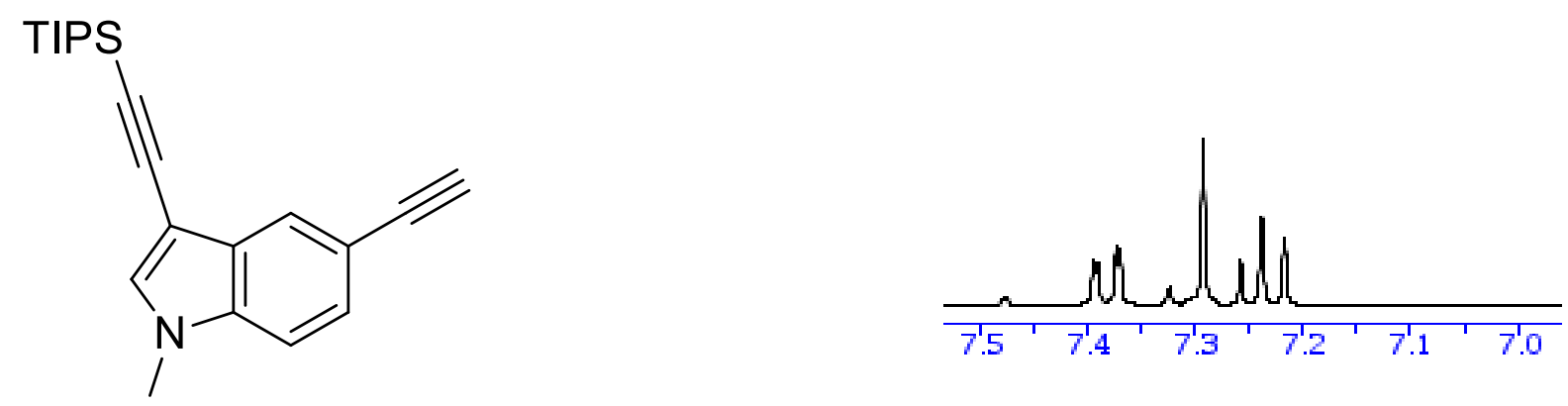

11
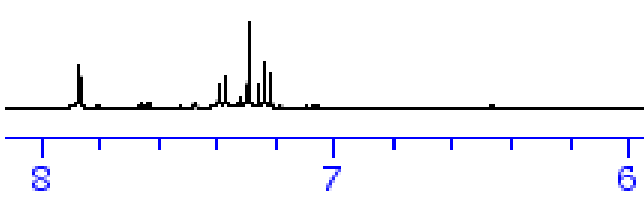

6

5 
solvent: $\langle\mathrm{CDCl} 3\rangle$

Frequency. $100.612769 \mathrm{MHz}$

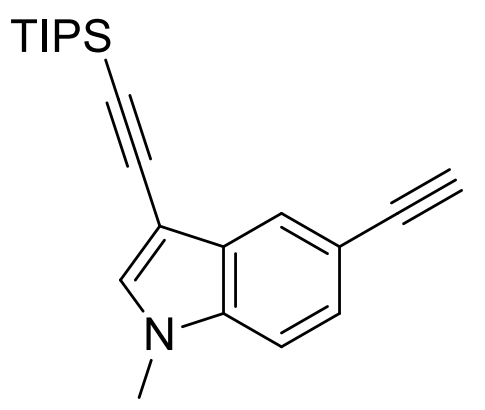

11

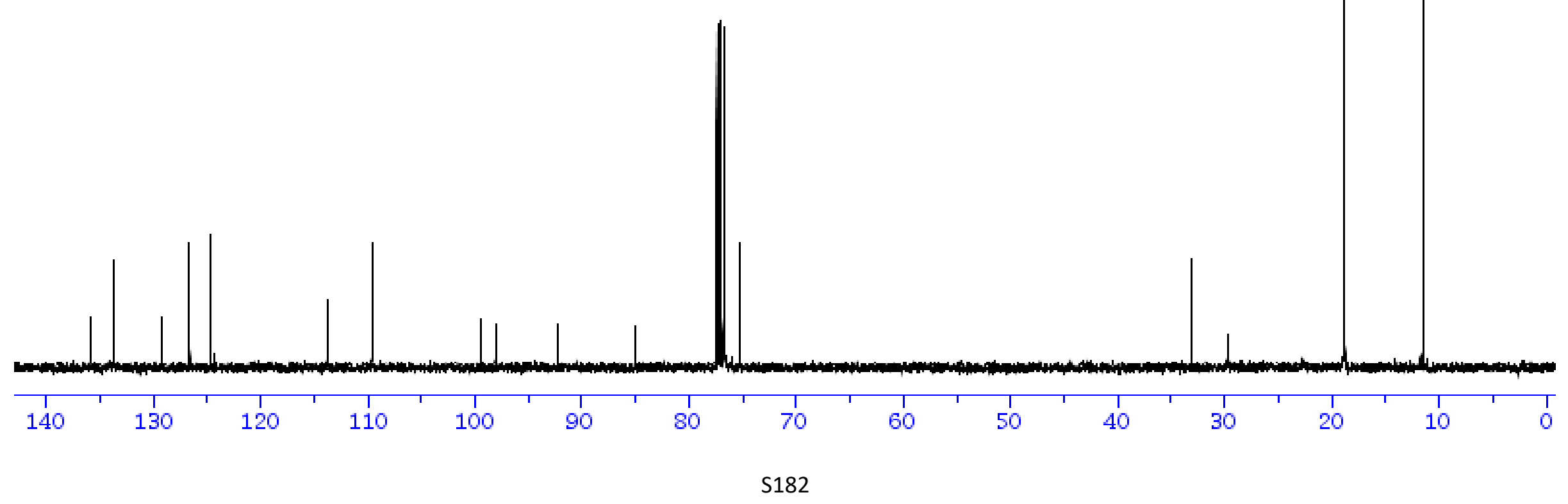




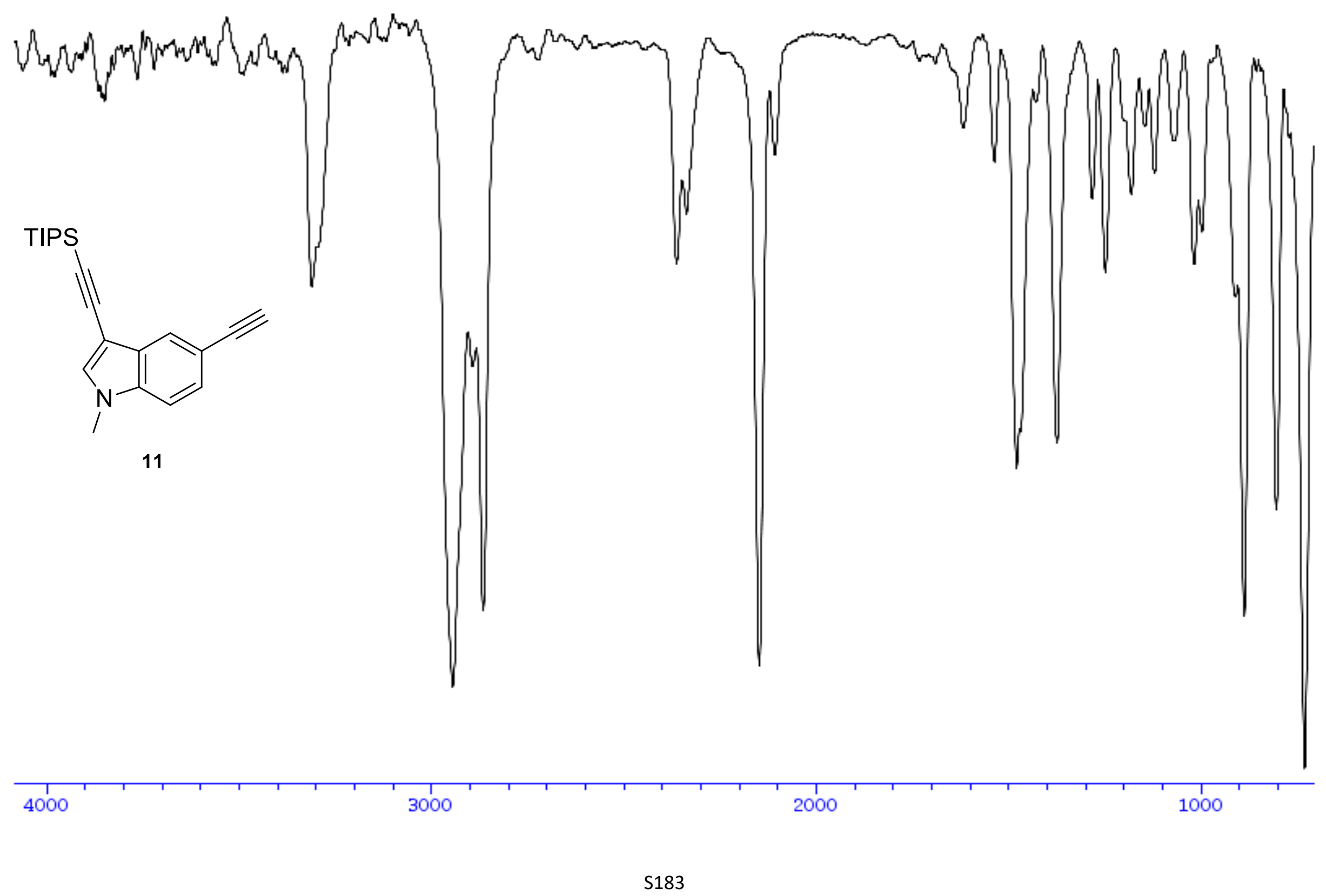


solvent: $\langle\mathrm{CDCI} 3\rangle$

Frequency. $400.13 \mathrm{MHz}$

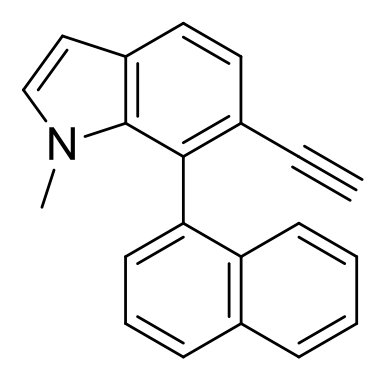

40
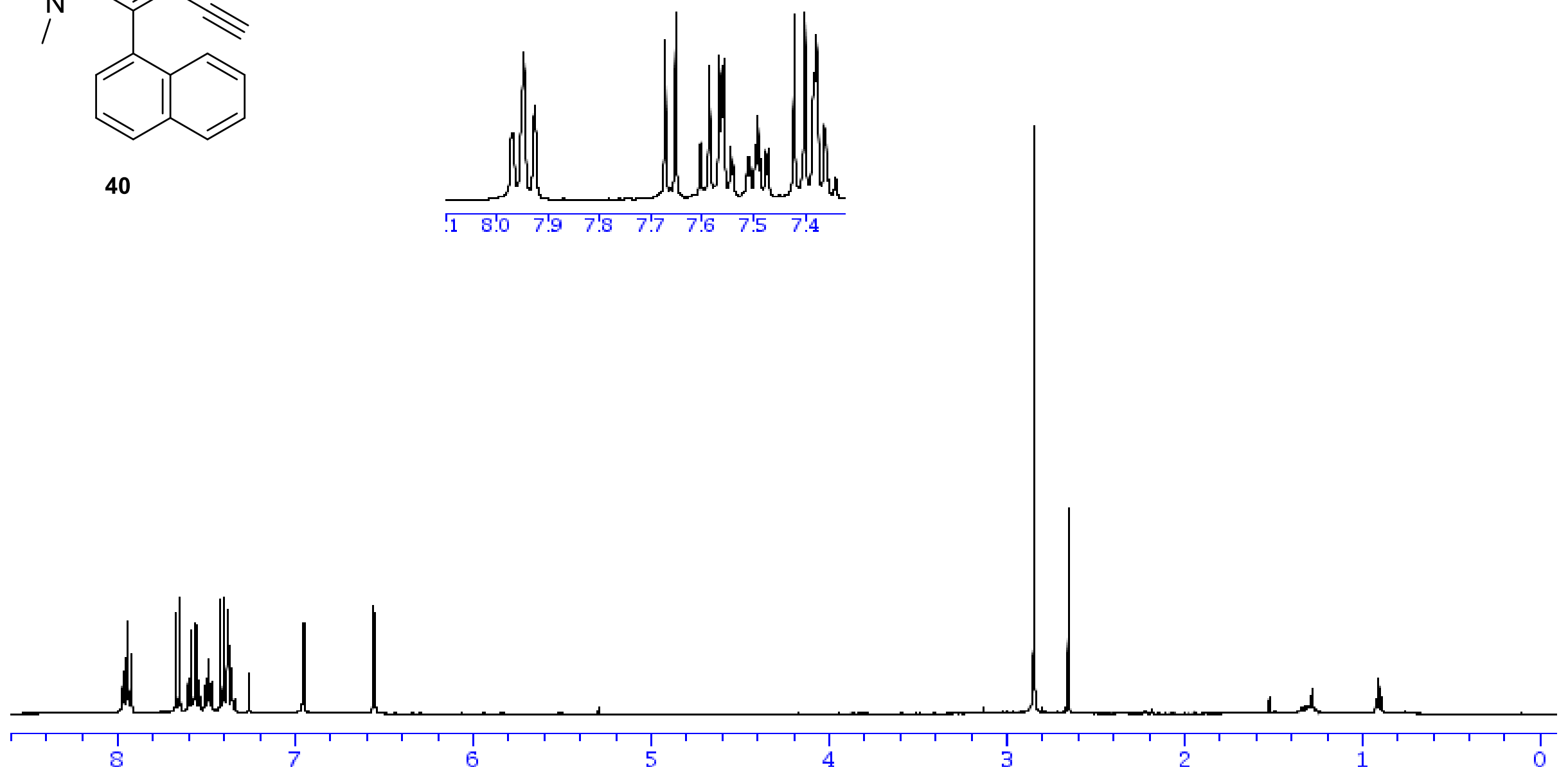
solvent: $<\mathrm{CDCI} 3>$

Frequency. 100.612769M Hz

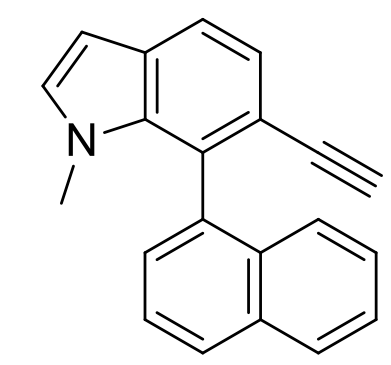

40

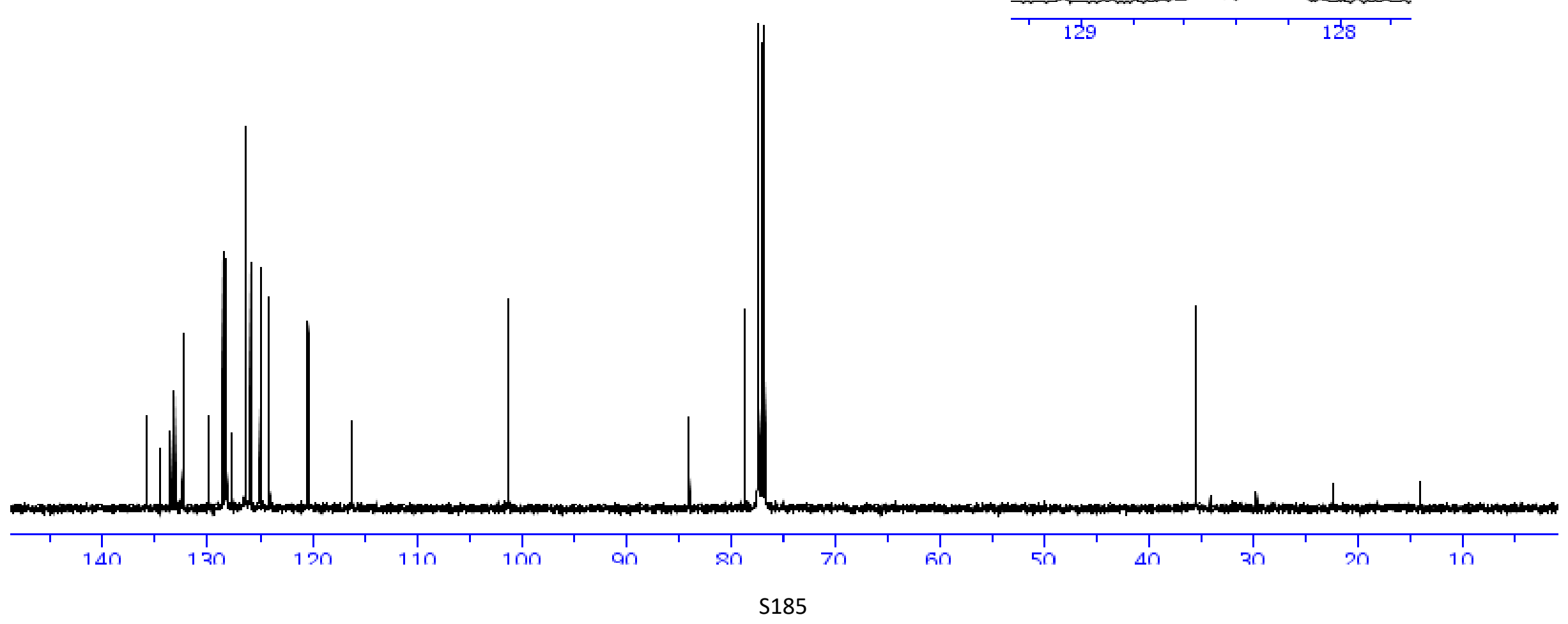




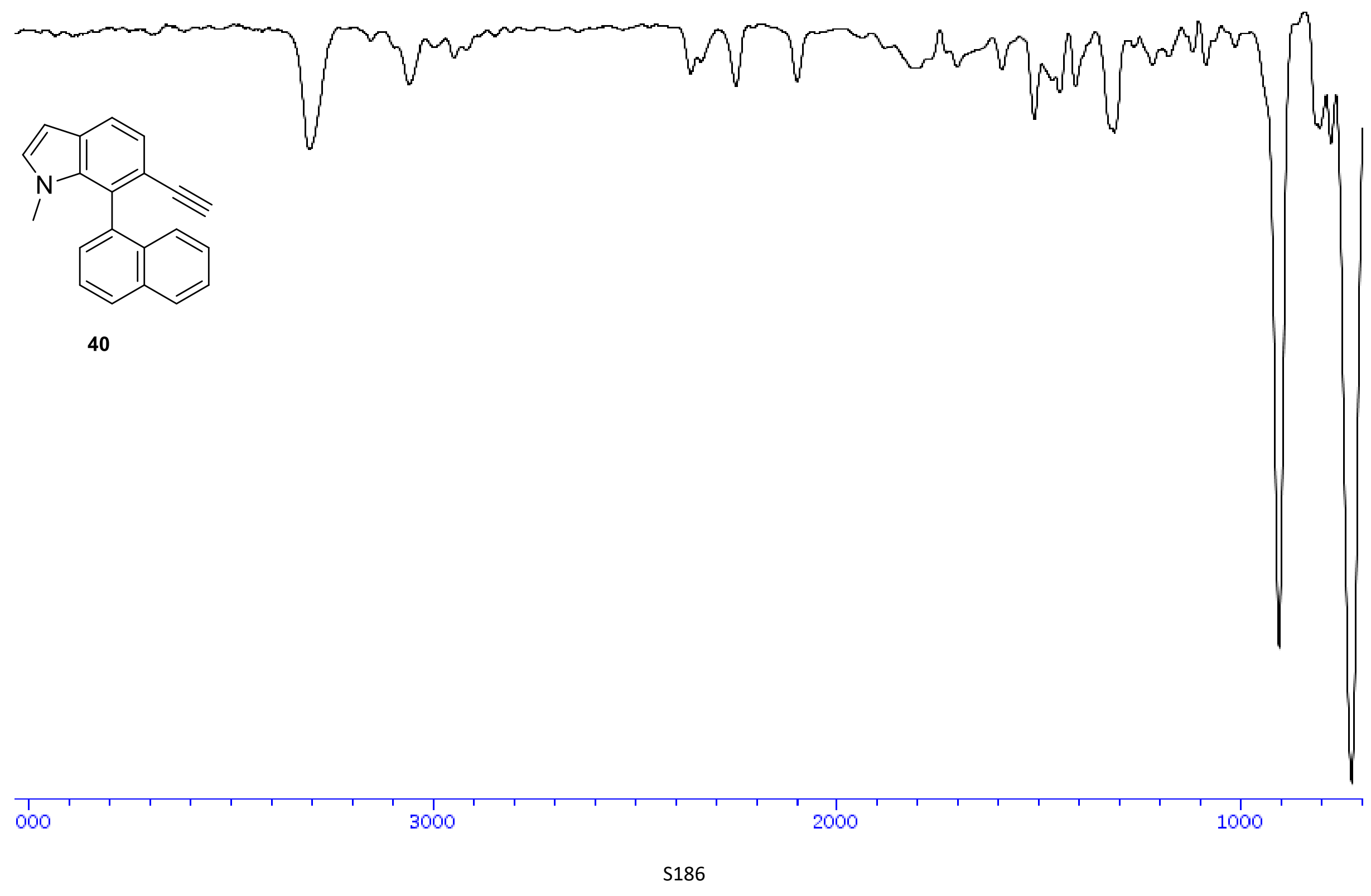


solvent: $\langle\mathrm{CDCl} 3\rangle$

Frequency. $400.13 \mathrm{MHz}$
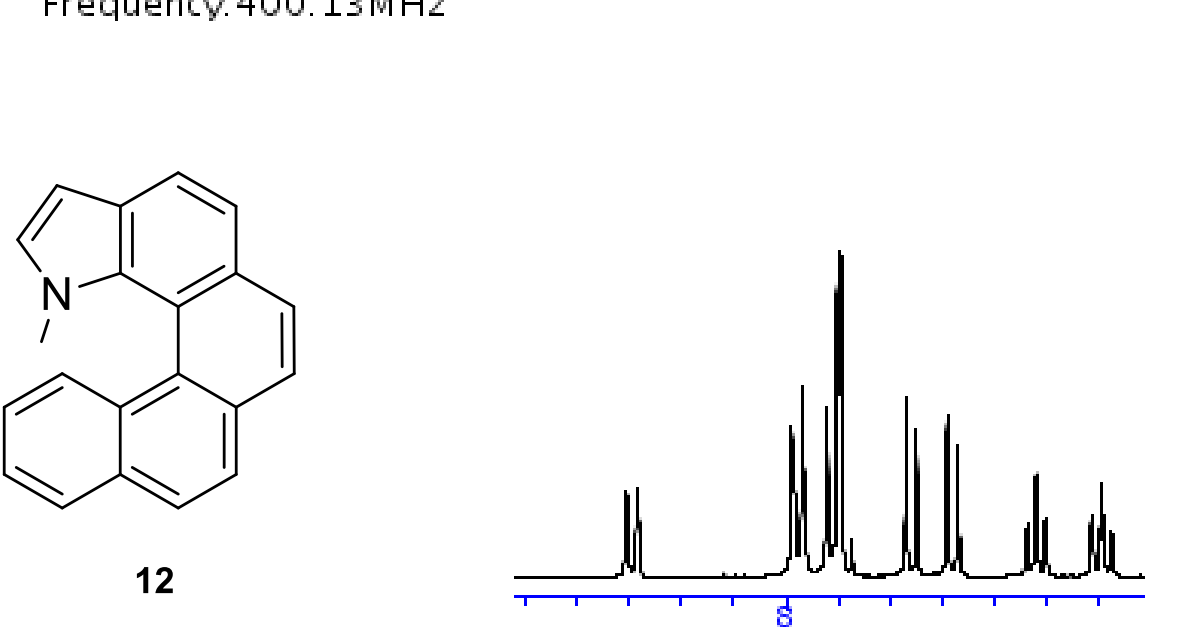

Illuall
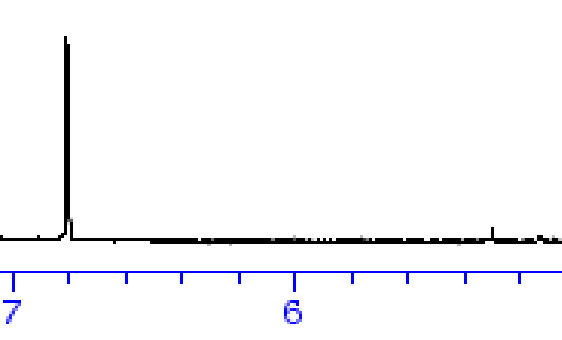

5 
solvent: $<\mathrm{CDCI}>$

Frequency. $100.612769 \mathrm{MHz}$

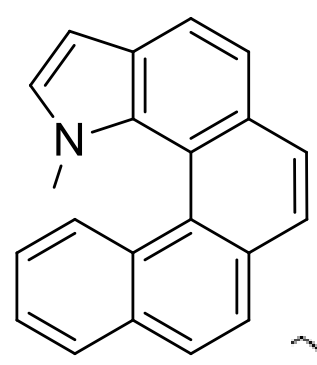

12
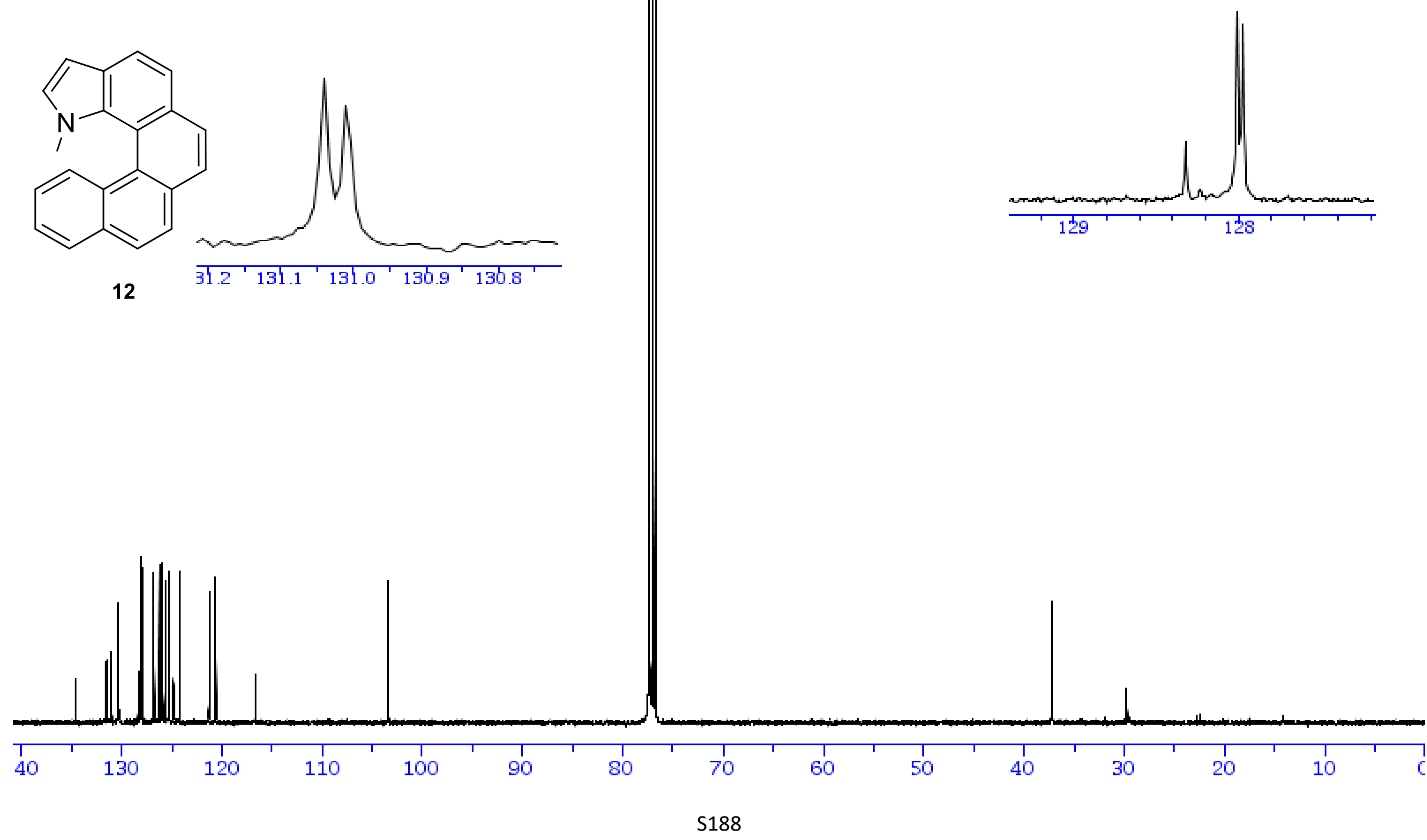


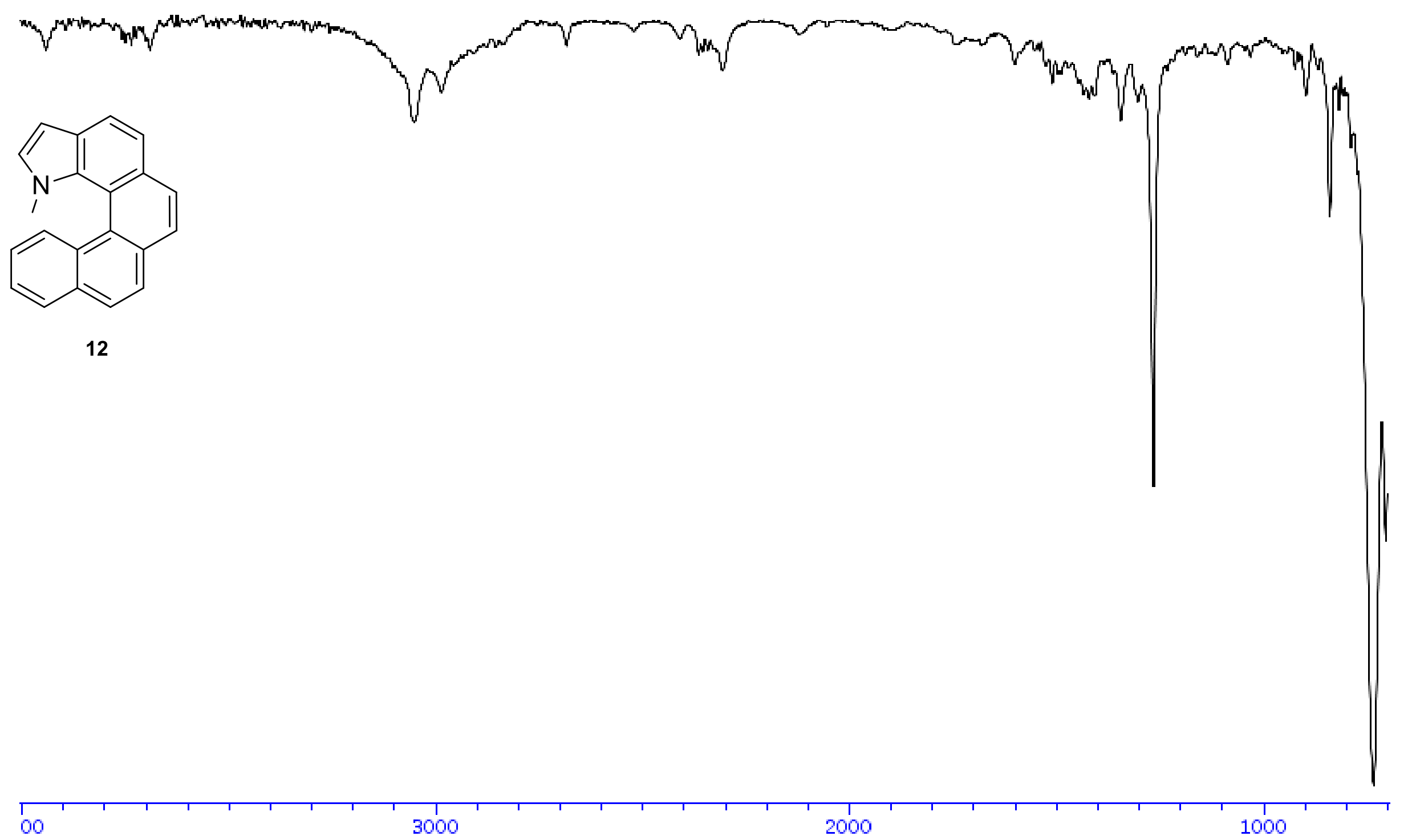

